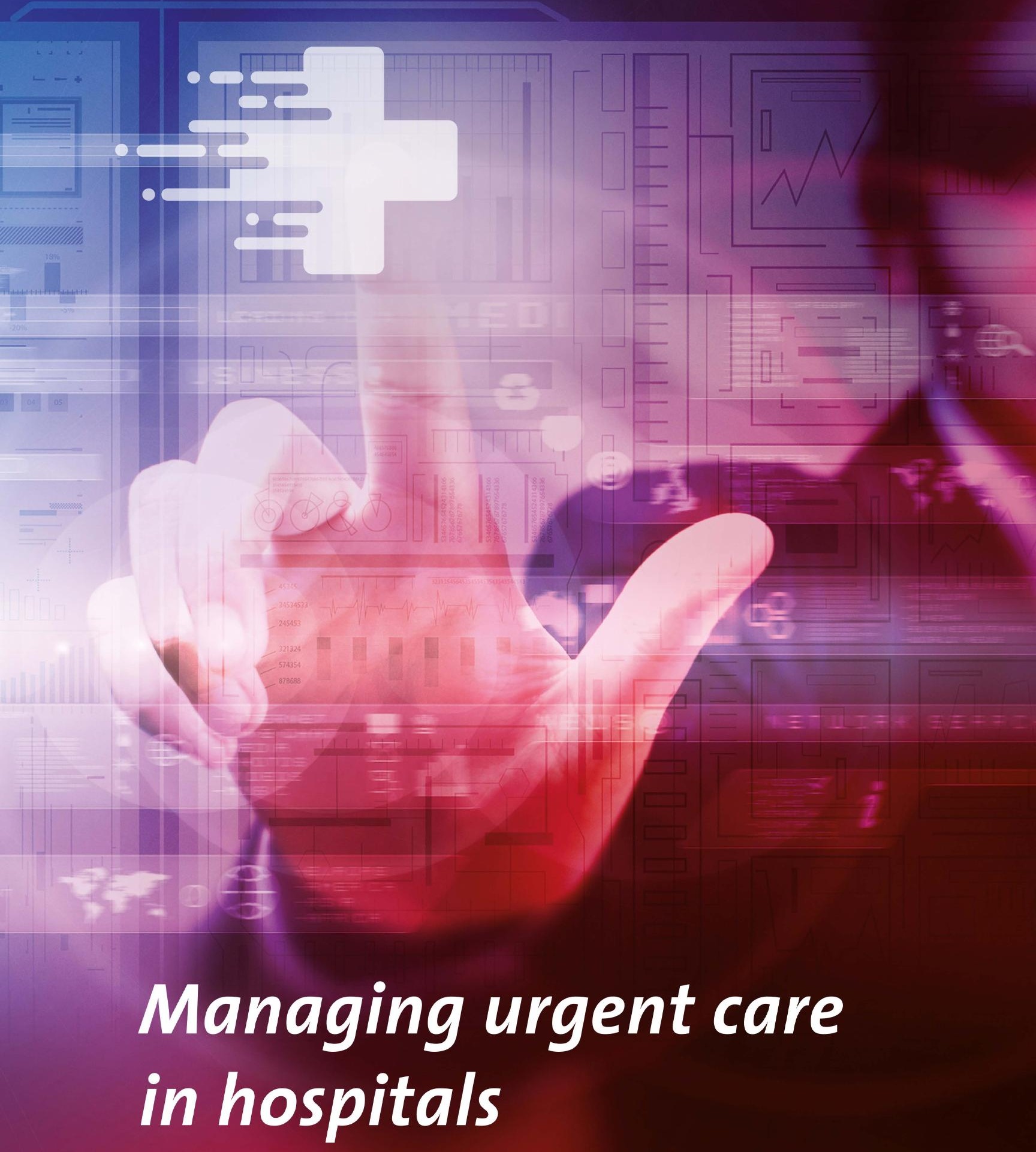

Nardo Jonathan Borgman 
MANAGING URGENT CARE IN HOSPITALS

Nardo Borgman 


\section{Graduation committee}

Chairman \& secretary: Prof. dr. T.A.J. Toonen

University of Twente, Enschede, the Netherlands

Supervisors: $\quad$ Prof. dr. R.J. Boucherie

University of Twente, Enschede, the Netherlands

Prof. dr. ir. E.W. Hans

University of Twente, Enschede, the Netherlands

Co-supervisor: $\quad$ Dr. ir. I.M.H. Vliegen

Eindhoven University of Technology, Eindhoven, the Netherlands

Referee:

Dr. M.J.A. Tasche

HagaZiekenhuis van Den Haag, The Hague, the Netherlands

Members: Ass. prof. C.J.M. Doggen

University of Twente, Enschede, the Netherlands

Prof. dr. J. van de Klundert

Erasmus University Rotterdam, Rotterdam, the Netherlands

Em. prof. dr. ir. J.J. Krabbendam

University of Twente, Enschede, the Netherlands

Prof. dr. C. Vasilakis

University of Bath, Bath, United Kingdom

Ph.D. thesis, University of Twente, Enschede, the Netherlands

Center for Healthcare Operations Improvement and Research

Beta Research School for Operations Management and Logistics (No. D208)

Printed by Ipskamp Printing

Cover design: Michael Thé

Copyright (c) 2017, Nardo Borgman, Enschede, the Netherlands

All rights reserved. No part of this publication may be reproduced without the prior written permission of the author.

ISBN 978-90-365-4360-6

DOI 10.3990/1.9789036543606 


\title{
MANAGING URGENT CARE IN HOSPITALS
}

\section{PROEFSCHRIFT}

\author{
ter verkrijging van \\ de graad van doctor aan de Universiteit Twente, \\ op gezag van de rector magnificus, \\ Prof. dr. T.T.M. Palstra, \\ volgens besluit van het College voor Promoties \\ in het openbaar te verdedigen \\ op vrijdag 23 juni 2017 om 14.45 uur
}

door

Nardo Jonathan Borgman

geboren op 21 december 1985

te Hoogeveen, Nederland 
Dit proefschrift is goedgekeurd door de promotoren:

Prof. dr. R.J. Boucherie

Prof. dr. ir. E.W. Hans 




\section{Voorwoord}

'Heb je er wel eens over nagedacht om na je afstuderen te gaan promoveren?', zonder deze vraag van jou Martijn was ik denk ik nooit op het idee gekomen. Uiteindelijk ben je niet mijn begeleider geworden maar tijdens het afstuderen heb je wel een zaadje geplant en daar wil ik je voor bedanken.

Meerdere keren in de afgelopen vierenhalf jaar heb ik gedacht: waar ben ik aan begonnen. Het was een intensieve tijd met veel ups en downs maar waarin ik ook ontzettend veel heb geleerd. Met een positief en trots gevoel kijk ik dan ook terug. En het eindresultaat is er. Een proefschrift met mijn naam erop, maar zonder vele anderen was het er nooit gekomen. Zonder af te doen aan de steun van iedereen die een bijdrage heeft geleverd, wil ik een aantal mensen in het bijzonder bedanken.

Erwin, ik bewonder je om je altijd positieve en vooruitkijkende blik. Tijdens mijn gehele traject kon ik altijd bij je terecht en met name het afgelopen jaar hebben we intensief samengewerkt. Je bent ongetwijfeld de meest positieve persoon die ik ken. Dit in combinatie met een goede dosis humor maar ook het geven van richting maakte dat ik na een gesprek met jou weer gemotiveerd was en de belangrijke dingen niet uit het oog verloor. Richard, met jou heb ik wat minder samengewerkt als tweede promotor maar op de momenten dat we bij elkaar zaten, kon ik dankzij jouw kritische blik altijd weer verder. Bedankt hiervoor.

Ingrid, jij was mijn begeleider en we hebben dan ook vele gesprekken met elkaar gevoerd. Je maakte altijd tijd voor me en was erg betrokken. Ik waardeer dat ik altijd open met je kon praten over hoe het ging. De duidelijkheid en sturing die je gaf, hielpen me in de goede richting. Bedankt daarvoor. Maartje Zonderland, ook jij bent nog even mijn begeleider geweest. Bedankt voor je tijd en de inzichten die je bracht tijdens de periode dat Ingrid met zwangerschapsverlof was.

Uiteraard een speciaal dankwoord voor de rest van mijn commissieleden. Carine, ons contact begon tijdens mijn afstuderen. Leuk dat je nu ook in de commissie zit waarmee ik mijn promotietraject afsluit. Marjolein Tasche, Joris van de Klundert, Koos Krabbendam and Christos Vasilakis: thank you for being part of my graduation committee.

De collega's van het HagaZiekenhuis in Den Haag wil ik ook graag bedanken. Ad, Fred en Arnoud, ondanks de soms rumoerige tijd kwam ik met plezier bij jullie werken. In het bijzonder wil ik Auke bedanken voor zijn begeleiding.

De afgelopen jaren voelde ik mij thuis bij meerdere groepen op de Universiteit Twente. Ik wil dan ook mijn CHOIR, IEBIS en SOR collega's bedanken 
voor de gezelligheid, adviezen en (zinloze) discussies. Maartje, Ingeborg, Sem, Gréanne, Joost, Thomas, Bruno en Shiya, bedankt voor de vele koffiemomenten, zinnige en onzinnige gesprekken en ondersteuning bij de wiskundevraag van de dag. Gréanne, uiteindelijk heb je mijn proefschrift meermaals doorgelezen op fouten en inconsistenties, bedankt hiervoor!

De collega's van de UT wil ik ook graag bedanken voor de leuke en bijzondere momenten die we samen beleefden tijdens congressen. In het bijzonder noem ik dan het congres van 2015 in Canada waar we met zijn allen in een grote Airbnb woning logeerden. Maartje en Sem, daarna zijn we nog een week door Canada getrokken. Iets wat je niet met iedere collega doet, maar mede door jullie is Canada een erg bijzondere ervaring geworden.

Als laatste wil ik mijn vrienden en familie bedanken voor hun steun en de zeer nodige afleiding op zijn tijd. Bastiaan en Stef bedankt dat jullie mijn paranimfen willen zijn.

Lieve Hilde, bedankt voor je altijd nuchtere blik en liefdevolle steun. Met jou heb ik altijd de ups en downs van mijn promotietraject kunnen delen en zonder jou had ik dit nooit af kunnen ronden. Zeker in combinatie met je zwangerschap is dat niet altijd makkelijk geweest. En kleine Abel, wat een indruk heb je de eerste dagen van je leven al op me gemaakt. Een extra motivatie om ook de laatste fase van mijn promotietraject af te ronden.

Nardo

Enschede, mei, 2017 


\section{Contents}

1 Introduction 1

1.1 Research motivation . . . . . . . . . . . . . . . . . 1

1.2 Organizing non-elective care in hospitals . . . . . . . . . . . 2

1.3 Operations research in healthcare . . . . . . . . . . . . 2

1.4 HagaZiekenhuis . . . . . . . . . . . . . . . . . . . 3

1.5 Thesis outline ...................... 3

2 Urgent care planning and scheduling in hospitals: A literature review

2.1 Introduction . . . . . . . . . . . . . . . . 5

2.2 Review scope and categorization .............. 6

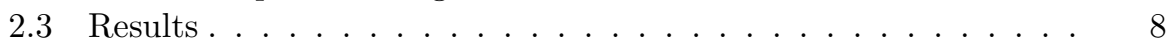

2.4 Discussion and conclusion . . . . . . . . . . . . . 23

3 Simulation and logistics optimization of an integrated emer$\begin{array}{ll}\text { gency post } & 27\end{array}$

3.1 Introduction . . . . . . . . . . . . . . . . . . . . 27

3.2 Literature . . . . . . . . . . . . . . . . . . . . . . . . 28

3.3 Problem description . . . . . . . . . . . . . . . . . . 29

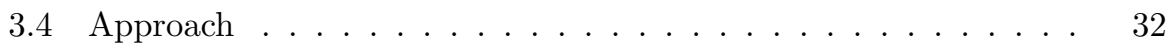

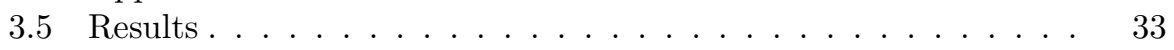

3.6 Conclusion ........................... 41

4 Appointment scheduling with unscheduled arrivals and reprioritization $\quad 43$

4.1 Introduction . . . . . . . . . . . . . . . . . . . . . . 43

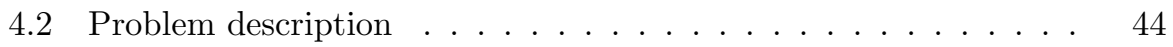

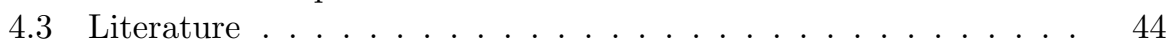

4.4 Assumptions and Approach . . . . . . . . . . . . . . . 46

4.5 Simulation model and search heuristics . . . . . . . . . . . . . . . 48

4.6 Experiments and results . . . . . . . . . . . . . . . 50

4.7 Discussion . . . . . . . . . . . . . . . . 59

5 Emergency OR or not: A simulation study of emergency surgery $\begin{array}{ll}\text { scheduling policies } & 69\end{array}$

5.1 Introduction . . . . . . . . . . . . . . . . . 69 
5.2 Literature . . . . . . . . . . . . . . . . . . . . . . . . . . . . . . . . 71

5.3 Problem Description . . . . . . . . . . . . . . . . . 73

5.4 Approach . . . . . . . . . . . . . . . . . . . . 74

5.5 Simulation model and OR Analyzer tool . . . . . . . . . . . . . . 77

5.6 Experiments and Results . . . . . . . . . . . . . . . 81

5.7 Conclusions . . . . . . . . . . . . . . . . . . . 92

6 Surgical procedure type scheduling incorporating semi urgent $\begin{array}{ll}\text { patients } & 95\end{array}$

6.1 Introduction . . . . . . . . . . . . . . . . . . 95

6.2 Literature . . . . . . . . . . . . . . . . . . . . . . . 96

6.3 Model formulation and solution approach . . . . . . . . . . . 97

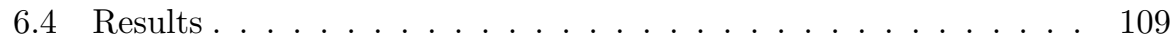

6.5 Conclusion . . . . . . . . . . . . . . . . . . . 118

$\begin{array}{llr}7 & \text { Conclusion and outlook } & 121\end{array}$

$\begin{array}{ll}\text { Bibliography } & 125\end{array}$

$\begin{array}{lr}\text { Acronyms } & 146\end{array}$

$\begin{array}{lr}\text { Summary } & 148\end{array}$

$\begin{array}{lr}\text { Samenvatting } & 152\end{array}$

$\begin{array}{lr}\text { About the author } & 156\end{array}$

$\begin{array}{lc}\text { List of publications } & 158\end{array}$ 


\section{Introduction}

\subsection{Research motivation}

There is tremendous pressure on healthcare organizations to deliver care, as demand for care increases both quantitatively and qualitatively, and healthcare costs increase. This requires hospitals to design and manage care processes more efficiently, while maintaining the current quality of care.

One of the challenges in hospitals is to take into account the many uncertainties that arise during the delivery of care. This is especially prevalent when providing care for non-elective patients who require treatment. Non-elective patients are those patients that have some degree of urgency, ranging from emergency patients that must be treated as soon as possible, urgent patients that must be treated within hours, and semi-urgent patients, who may wait for several days. Typically there is variability in the arrival of these non-elective patients, as well as varying degrees of acuity that make planning for these patients difficult yet important in order to ensure timely access. As urgency increases, the stakes involved increase for both patients and healthcare providers. Clearly, there is less margin for error when managing non-elective care, as consequences of tardy treatment can be serious.

Non-elective care is prevalent throughout hospitals. In the Netherlands, around half of all patients submitted to wards, as well as half of all inpatient days of hospitals are patients that originated from the Emergency Department (ED) [190]. In addition, $15 \%$ of ED visits lead to surgery in the Operating Room (OR), and around $40 \%$ of the Diagnostic Treatment Combination (DTC) costs stem from the ED [235]. Moreover, there are (semi-)urgent patients requiring further treatment that may be referred from outpatient clinics. The question then is how to efficiently organize healthcare processes that are able to properly adapt to the specific needs and characteristics of non-elective patient demand.

In order to accommodate the uncertainty inherent to non-elective care, an increased flexibility in resources' planning and control is required. This thesis focuses on designing care processes and developing planning and control approaches that take into account non-elective patients in addition to elective patients. For example, by reserving an OR for non-elective arrivals, (some of) these patients can be treated in a timely manner. The flexibility requirement results in making 
a trade-off between access for non-elective care and efficiency. Clearly, a high utilization of resources reduces the flexibility for non-elective care. Examples of redesigning care processes are the integration of ED and General Practitioners Post (GPP) in an Integrated Emergency Post (IEP), and the use of 'fast-track' pathways in an ED where some resources are dedicated to lower urgency patients.

\subsection{Organizing non-elective care in hospitals}

The research presented in this thesis aims to support decision making in hospitals regarding the planning and control of healthcare processes involving non-elective patients, such that patients are treated effectively and in a timely manner, and hospital resources are efficiently utilized. There is not only uncertainty in the arrivals of non-elective patients, but also in the care pathways that are followed throughout the hospital once a patient arrives. As such, a non-elective patient's pathway often falls along multiple departments and does not stop at the ED. For example, after arriving at the ED, diagnostics may be requested from the radiology department, after which admission to a ward is required. Therefore, all departments in the non-elective patient pathway need to organize their resources to be able to provide care for non-elective patients in a timely manner, which often conflict with elective patients performance indicators. For example, to reduce the waiting time for non-elective patients at the OR, additional time may be reserved for these patients. Consequently, there is less time available for elective patients, and their access time may increase. Elective and non-elective patient planning may affect each other even across departments. For example, by increasing OR capacity for elective patients, more beds at the wards are used for elective patients, which in turn may lead to congestion at the ED as admitting patients to wards becomes more difficult.

In order to improve the planning and control of healthcare processes involving non-elective patients, we focus on solution approaches using operations research techniques that account for both elective and non-elective patients. A trade-off is sought between performance indicators of both elective and non-elective patients, as well as a trade-off between efficient and effective care.

\subsection{Operations research in healthcare}

Operations research as a scientific approach aims to help in decision making by employing advanced analytical methods. To improve decision making, operations research offers a variety of techniques and methods, such as mathematical modeling, queuing theory, statistical analysis, and computer simulation [117]. Operations research originated from the military planning during World War 2, and since the 1950s operations research has been applied to healthcare and resulted in significant contributions in accomplishing essential efficiency gains in healthcare delivery [114]. 
With the use of operations research methods, healthcare professionals may be supported in decision making that allows for improvements in efficiency in the delivery of healthcare, while offering appropriate quality of care. In addition, possible changes in healthcare processes may be modeled and evaluated safely, without experimenting with interventions in practice - which may have adverse effects.

\subsection{HagaZiekenhuis}

The studies in this thesis are largely inspired by problems encountered at the HagaZiekenhuis (Haga) in The Hague. Haga is one of the largest teaching hospitals in The Netherlands, and as a top-clinical hospital hosts almost all medical specialties and offers specialized care. In 2015, Haga employed 3,569 (2,872 fte) staff members, and hired 199 medical specialists. The hospital has 590 beds, provided 202,018 (initial) outpatient clinic consultations, 30,717 patient admissions, and had 54,959 ED visits [95]. Haga focuses on the following core values: considerate care, innovation, and cooperation. Haga aims to deliver high quality care in a timely manner, and centers its care around patient needs and wants, high quality service and freedom of choice [96]. Although the research in this chapter is applied to specific cases, most of the methods used are generic and may be readily adapted to other hospital or healthcare settings.

\subsection{Thesis outline}

Chapter 2 provides an overview of the non-elective care planning and scheduling literature within hospitals, and categorizes the literature according to departmental focus, hierarchical planning level, urgency classification, and chosen methodology. The aim of the chapter is to gain insights in how to best structure and plan non-elective care processes, and identify research opportunities.

Chapter 3 uses computer simulation and a heuristic approach to find the optimal process design of an integrated emergency post, the merger between emergency department and general practitioners post. The chapter systematically evaluates and compares possible interventions that consist of staff allocations, resource allocations, and process changes. In the design and evaluation of interventions, patient preferences are taken into account.

Chapter 4 proposes a methodology to optimize appointment schedules for elective patients at a radiology department that also services semi-urgent patients. The appointment schedule dictates the times during the day in which appointments may be scheduled. By taking into account non-elective patient arrival and duration characteristics, the appointment schedule may be optimized such that waiting times for both elective and non-elective patients improve. Our 
methodology uses computer simulation, Markov processes, and heuristics.

Chapter 5 studies multiple policies for operating theaters to allocate operating room (OR) capacity to emergency patients, such as the use of dedicated emergency ORs. We identify key patient and hospital characteristics, such as patient case mix and hospital size, that have an effect on the policies. The policies are then evaluated under multiple case (hospital) characteristics. The analysis is carried out using computer simulation, and results are integrated in an interactive tool.

Chapter 6 provides a methodology to create surgery type schedules that incorporate elective patients and semi-urgent patients that must be seen within several days. A surgery type schedule determines the number, and type, of surgeries that are planned in an OR, to which at a later point in time surgical cases are assigned. By optimizing the surgery type schedule, we minimize the number of required ORs to treat all patients, and ensure timely access for semi-urgent patients. Our approach uses integer linear programming, combined with sample average approximation and column generation. 


\section{Urgent care planning and scheduling in hospitals: A literature review}

\subsection{Introduction}

Ideally, healthcare providers are able to provide care in a timely manner for all patients. However, aside from elective patients, there are often also non-elective patients requiring medical care. Non-elective patients are those patients that have some degree of urgency, ranging from emergency patients that must be treated as soon as possible, to semi-urgent patients, who may wait for several days. Typically there is variability in the arrival of these non-elective patients, as well as varying degrees of acuity that make planning for these patients difficult yet important in order to ensure timely access.

The importance of planning of non-elective patients is mentioned in the literature [40,89], yet more often than not non-elective patient flows are not taken into account and the focus is entirely on elective patient flows. This chapter aims to provide insights into the question how to plan, schedule and control processes within a hospital setting to best account for non-elective patients. We provide a structured overview of the literature on capacity planning and control decisions that take place in hospitals, and which specifically take into account urgent patient classes.

Our contribution is twofold. First, we provide an overview of non-elective care planning and control literature within hospitals. With a hospital-wide scope we do not limit our overview to a single healthcare service (e.g., emergency departments), or methodology (e.g., discrete event simulation). Second, we analyze and categorize the found literature, and we outline open research questions.

This chapter is organized as follows. Section 2.2 describes our review scope, search strategy, and categorization of the literature. Section 2.3 identifies, classifies and discusses the found literature. Section 2.4 contains a discussion of our findings. 


\subsection{Review scope and categorization}

In this section we detail the scope of our review of capacity planning and control of non-elective hospital care, our search strategy, and we describe our categorization of the literature.

\subsubsection{Scope}

We limit our scope to papers that use OR/MS methods to model and quantitatively assess patient related processes that take place within a hospital setting. We not only include the emergency department, but also other departments that offer their services to to non-elective patients such as diagnostics facilities and operating theaters. Also, we include and distinguish between different urgency levels. We exclude the closely related field of emergency medical services planning (EMS), which focuses on the planning, scheduling, allocation and relocation of medical services such as ambulances, helicopters, and emergency response field locations, as well as forecasting emergency demand $[11,116]$. Multiple recent reviews on EMS can be found in the literature [11, 21, 38, 82, 86, 105, 116, 165].

\subsubsection{Search strategy and data collection}

We access academic literature through the electronic database Web of Science (https://webofknowledge.com/), as well as the ORchestra (http://www.choirut.nl/search-orchestra) database. ORchestra contains literature within the scope of OR/MS in healthcare and is updated and maintained by the Center for Healthcare Operations Improvement and Research (CHOIR), a research group of the University of Twente. Within ORchestra, references are categorized by both medical and mathematical topics, as well as hierarchical planning levels. Within Web of Science we use the subject categories Operations Research Management Science and Health Care Sciences Services.

We searched the aforementioned databases for papers published since 2000, written in English, containing combinations of relevant keywords describing the use of OR/MS in hospitals specifically incorporating non-elective patients. Table 2.1 contains the search strategy used to produce the set of initial articles. Both peer-reviewed papers and conference proceedings are included in the review. Papers containing the search terms in title, abstract, or keywords were included. The term sets are used to identify papers applied to a hospital setting, mentioning emergency characteristics, and that also apply OR/MS methodologies.

Following the database search, the initial set of papers is selected for abstract review based on title and keywords. In the next stage the abstracts were read and a further selection was made for inclusion in the final set of papers, which were read in full. Figure 2.1 displays an overview of the search strategy and remaining papers after each step. In total, 3,399 papers related to non-elective care are reviewed, of which 164 are included in the final set of papers. 
Figure 2.1 Overview of the review approach

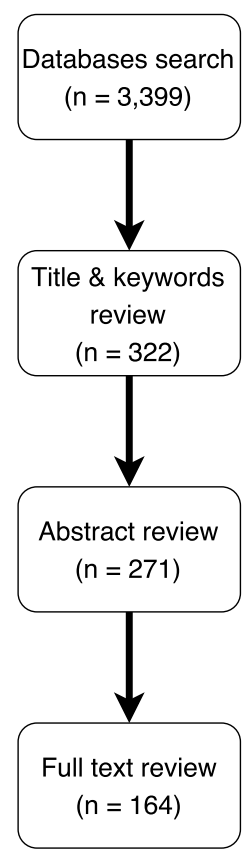

\subsubsection{Categorization}

We categorize the literature using the following categorizations:

- Departmental focus (2.3.1)

- Hierarchical planning level (2.3.2)

- Urgency classification (2.3.3)

- Methods (2.3.4)

The departmental focus categorizes the literature according to the hospital departments that are being considered when modeling non-elective care, as these may have their distinct problems regarding non-elective patients. We categorize the literature along the following four departments: Emergency Department (ED), Operating Theater (OT), wards, and ambulatory services. Both the wards

Table 2.1 Search strategy

Used search strategy

$\mathrm{TS}=(($ healthcare OR health care OR hospital OR patient) AND (acute OR

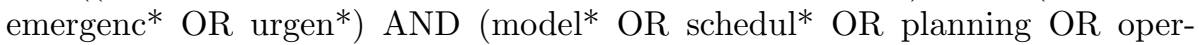
ation* OR optim* OR improv*)) AND SU=((OPERATIONS RESEARCH MANAGEMENT SCIENCE) OR (HEALTH CARE SCIENCES SERVICES))) AND LANGUAGE: (English) 
and ambulatory services categories contain multiple, possibly specialized, hospital departments (e.g., Intensive Care Units (ICU) and radiology department diagnostics). We include the wards and ambulatory services as single department types as the research problems encountered at both are often very similar. In addition, we identify papers that incorporate more than one hospital department, and study (part of) non-elective patient pathways through the hospital.

For the hierarchical planning levels we distinguish between the strategic, tactical, offline operational, and online operational planning levels [102, 114]. The strategic planning level contains the long-term decision making involved when designing, developing and dimensioning healthcare processes. Examples of strategic planning are the acquisition of resources (e.g., diagnostic machines) or determining a new facility's location. The tactical planning level involves medium-term decision making that addresses the organization of the healthcare processes after strategic decisions are made. For example, after deciding on the number of diagnostic machines, at the tactical level block schedules may be created that allocate time capacity to different patient groups. Finally, the operational planning level involves the short-term decision making when executing the healthcare delivery process. At this level, there is little flexibility as strategic and tactical level decisions have limited the scope for operational level decision making. The offline operational level denotes the in advance planning, such as scheduling appointments or staff. The online operational planning level consists of the control mechanisms that deal with monitoring the health care processes and reacting to unanticipated revents. An example of online operational planning is the scheduling of add-on surgical cases or emergencies [102, 114].

The urgency of the non-elective patients studies may have a considerable effect on the appropriate planning and scheduling approach. Therefore, we also categorize the literature along (patient) urgency and prioritization. Finally, we categorize the literature according to the used methodologies.

\section{$2.3 \quad$ Results}

\subsubsection{Departmental focus}

In this section we provide an overview of hospital departments that are considered when modeling non-elective care. In addition, we evaluate the multidepartmental studies that are carried out.

Figure 2.2 gives an overview of the research carried out per hospital department. As may be expected, the most studied hospital department is the emergency department, with 92 papers. The second most studied department is the hospital ward $(n=52)$. Various types of wards are evaluated, like critical care units [45, 50, 87], Medical Assessment Units (MAU) [51, 150, 193, 194], and Post Anesthetic Care Units (PACU) where patients recover after surgery [226]. The third most studied department is the OT $(n=36)$. Here the focus lies on the scheduling of elective patients, while accounting for unscheduled urgent patients 
that may arrive. The proportion of urgent patients that arrive at operating theaters is often omitted, however it seems to vary considerably, ranging from $5 \%$ of patients that must be seen within hours [8], to $85 \%$ of patients originating from the ED [262]. The fourth most studied department is ambulatory services $(n=22)$. Ambulatory services provide care to (urgent) patients without offering a room and/or bed to patients. Examples of ambulatory care services used by urgent patients are laboratories that carry out tests [16, 204], outpatient clinics [73, 141, 229, 234], and imaging diagnostics facilities (e.g., CT scanners) $[35,79,84,143,172,215,240]$. Table 2.2 lists the papers per hospital department considered. Note that papers that model more than one hospital department are listed multiple times. The topics that are studied at the various departments are discussed along with the hierarchical planning levels in the next section.

Figure 2.2 Number of publications per hospital department $(\mathrm{n}=92+52+36+22$ = 202)

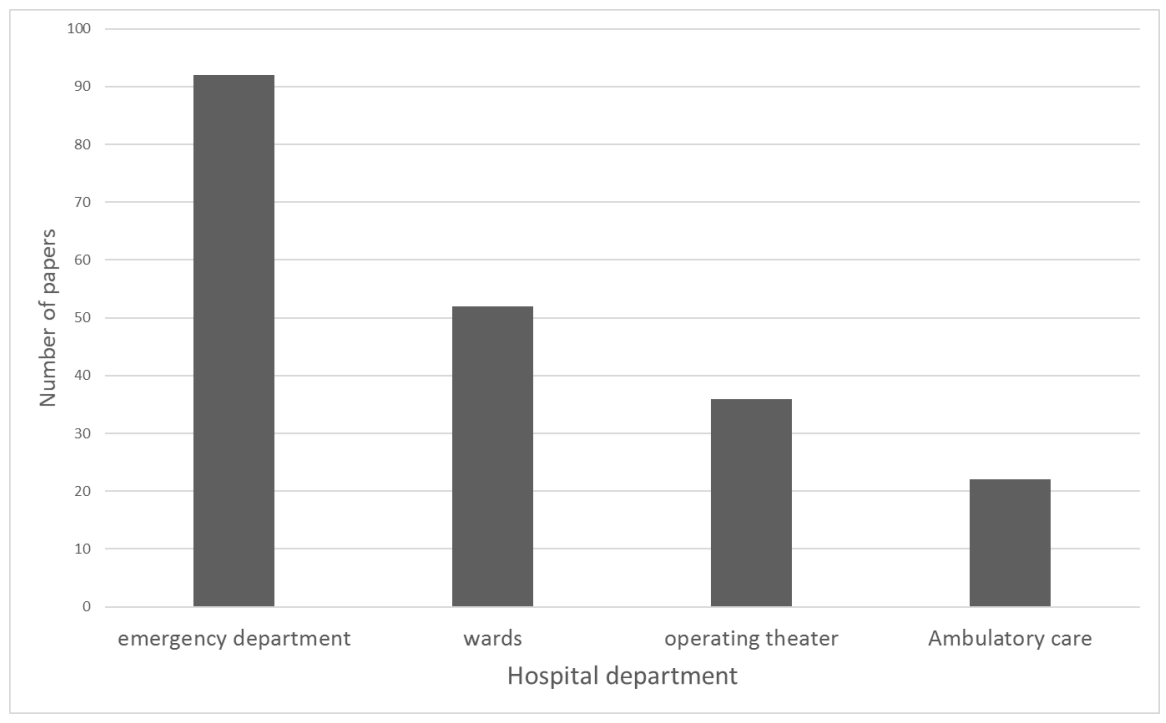

There are also papers that (partially) include more than one hospital department. In total there are 29 papers that include two or more departments. When more departments are included, we find that one department is the primary focus, and to some extent aspects of a second department are included when these influence performance. The exception are studies that model non-elective patient pathways across the entire hospital (e.g., [33, 90, 157]). For example, [147] present an analytical approach to predict bed census on wards taking into account the Master Surgery Schedules (MSS) from the operating theater, as well as arrival rates from the ED. The arrival rates from the ED are considered as input however, and no further ED details are modeled. Table 2.3 gives an overview of the research that encompasses more than one hospital department. The most common combination (18) is the modeling of emergency department and wards. In 
Table 2.2 Publications per department $(\mathrm{n}=29)$

\begin{tabular}{ll} 
Department & References \\
\hline Operating theater & {$[2,8,17,18,32,61,63,64,68,77,93,106,113,125,126$,} \\
& $147,153-156,171,184,192,195,199,201,207,225-$ \\
& $228,247,249,253,262,263]$ \\
Wards & {$[2,17,18,18,18,22,24,33,37,37,37,45,50,51,61,71$,} \\
& $81,83,87,90,97,103,103,104,109,125,125,133,142$, \\
& $147,149-151,157,166,169,171,175,180,193,194$, \\
& $206,213,217,226,231,237,242,245,246,258,260]$ \\
Emergency department & {$[1,3,5,12-14,19,22,23,29,33,36,39,44,47-49,51-$} \\
& $53,55,58,59,62,71,72,74,85,90,92,97-99,107,108$, \\
& $111,118,121,122,124,127,130-132,136,137,142$, \\
& $144,145,148,152,157,159,161,163,164,166,173-$ \\
& $176,179,180,183,188,191,205,206,208-213,216$, \\
& $217,220,221,224,231,232,237,243,244,246,250$, \\
& $251,255,256,258,259,261]$ \\
& {$[16,33,35,73,79,84,90,112,120,123,141,143,157$,} \\
& $172,174,202,204,215,229,234,240,260]$
\end{tabular}

these papers, the effect of ward sizes on congestion levels at the ED is evaluated. For example, [166] use a queuing approach to model both ED and downstream inpatient units. They link the size of inpatient units, and patient length of stay to the required capacity at the ED, and suggest threshold sizes for inpatient units, after which additional capacity has no effects. Also, [97] model the implementation of a neurovascular unit in an acute hospital using simulation. They model both ED and wards, and evaluate the effect of having a specialised neurovascular unit comprised of beds of other wards evaluating a pre- and post situation they find a length of stay decrease of $8 \%$ with the same capacity available. Discharge policies are also a topic of research. [217] model an ED and inpatient wards to gain insight into waiting time reductions. They find that a discharge policy that focuses on better discharging patient throughout the day such that boarding and waiting times at the ED are reduced. [51] alternatively evaluate proactive discharge strategies using simulation, where patients are not only discharged based on medical conditions, but also based on ED resource utilization and number of boarders (i.e., patients waiting for further admission) at the ED. They found that discharging patients earlier when the ED and wards are busy resulted in improved ED performance, however they also note a likely increase of re-admissions.

Wards are also investigated together with the use of ORs. Non-elective patients not only require surgery, but are also subsequently admitted to a hospital ward. Trade-offs are sought between the utilization of wards and beds, and the blocking probability of a ward (i.e., the probability a patient can not be admitted). Both more specialized wards such as PACUs [18, 61, 226] and medium and intensive care units (ICU) $[2,17,18,125,169]$ are considered, as well as regular wards $[18,147]$. When wards are included, the recovery of patients after surgery 
Table 2.3 Studies incorporating aspects of multiple departments

\begin{tabular}{|c|c|c|}
\hline & OT & ambulatory \\
\hline \multicolumn{3}{|l|}{ ED } \\
\hline \multicolumn{3}{|l|}{ OT } \\
\hline Wards & $\begin{array}{l}{[22,33,51,71,[2,17,18,61,} \\
90,97,142,125,147,171, \\
157,166,175,226] \\
180,206,213, \\
217,231,237, \\
246,258]\end{array}$ & \\
\hline Ambulatory & $\begin{array}{l}{\left[\begin{array}{r}29, \\
157, \\
174]\end{array}\right.}\end{array}$ & $\begin{array}{l}{[33,90, \quad 157,} \\
260]\end{array}$ \\
\hline
\end{tabular}

is modeled alongside the OR, and patients must be scheduled such that not only an OR is available, but also a post surgery bed. For example, [226] evaluates the use of emergency and dedicated ORs together with PACU beds available. Several elective, emergency and PACU bed configurations are considered and optimized with regards to occupancy and overtime. Almost all papers that study both wards and operating theater primarily focus on modeling of the ORs. A different focus is used by [17], who model a neurosurgical ICU, and include the OR in their simulation model. They evaluate changes in available ORs, as well as staff and resources and its effect on the ICU, and find the combination of ORs and ICU beds that performs best with regards to waiting time, postponement, and medical criteria such as mortality rates.

Ambulatory services that are modeled simultaneously with EDs are laboratory and and radiology diagnostics [29, 174]. These diagnostic facilities also offer their services to elective patients, which may result in waiting times when diagnostics are requested from the ED. Specifically modeling these diagnostics may offer insights into the effects diagnostic availability has on ED length of stay, and possible interventions such as offering diagnostics at the ED [174]. Applied to wards, this effect of waiting for and undergoing diagnostics is also investigated. [260] models an emergency ward and recurring service processes such as reviewing diagnostic tests using a queuing network and evaluate staffing policies.

Despite the noted need to take into account patient flow and interaction between departments, most studies focus only on the ED (56\%). Most ED studies focus on capacity dimensioning and allocation problems, where resources and staff are allocated in order to minimize waiting times. The other departments where non-elective care is offered also treat elective patients, and limited attention is given to non-elective patient planning and scheduling, as this is intrinsically more difficult, especially for the operating theater where elective patients must be scheduled well in advance. If multiple departments are taken into account, this is mainly done through patient flow modeling (61\%), for example by modeling the entire care pathway of patients through ED and wards (e.g., [33, 97, 166]). As there are limitations to modeling approaches, direct model expansions to include 
additional process steps may be difficult. However, to gain insights into the interaction between hospital departments it may be sufficient to only include key components (e.g., [17, 147, 174]).

\subsubsection{Hierarchical planning level}

In addition to the type of department that is investigated, the planning level greatly influences the type of decision making that is focused on. Consistent with healthcare literature we identify the strategic, tactical and operational level of decision making $[102,114]$. At each planning level, decisions are made that frame the underlying planning level. Note that some papers are not categorized to a planning level, as these studies focus on forecasting and predicting aspects such as future emergency arrivals, and do not directly incorporate these models into decision making. Table 2.4 lists the publications per department and planning level.

Table 2.4 Publications per department and planning level

\begin{tabular}{ll}
\hline & Emergency department \\
\hline strategic & {$[33,49,90,107,157,159,209,213,237]$} \\
tactical & {$[1,3,5,12,13,19,22,23,29,33,36,39,44,51,55,58,62,71,74$,} \\
& $85,90,92,97-99,108,118,121,122,124,131,132,136,142,144$, \\
& $145,152,159,161,166,174-176,179,183,188,191,205,208,210-$ \\
& $212,216,217,220,221,224,244,246,250,251,255,258,259,261]$ \\
operational & {$[47,48,52,53,58,59,72,111,132,137,148,164,173,180,206$,} \\
& $231,232,261]$ \\
N/A & {$[14,127,130,163,243,256]$} \\
\hline & Operating theater \\
\hline strategic & {$[68,199,225,226,253,263]$} \\
tactical & {$[2,8,17,18,68,93,125,147,171,195,225-228,249,262,263]$} \\
operational & {$[2,18,32,61,63,64,77,93,106,113,126,153-156,184,192$,} \\
& $201,207,247,249]$ \\
N/A & Wards \\
\hline & {$[33,90,157,213,226,237]$} \\
strategic & {$[2,17,18,22,33,37,37,50,51,71,81,83,90,97,103,109,125$,} \\
tactical & $142,147,150,151,166,169,171,175,193,194,217,226,245$, \\
& $246,258,260]$ \\
operational & {$[2,18,24,45,61,87,104,109,133,180,206,231]$} \\
N/A & {$[149,242]$} \\
\hline & Ambulatory care \\
\hline strategic & {$[33,90,157]$} \\
tactical & {$[33,35,73,79,90,120,123,141,143,174,204,215,260]$} \\
operational & {$[16,73,84,112,143,172,202,229,234,240]$} \\
N/A & \\
\hline
\end{tabular}




\section{Strategic planning}

At the strategic level, long-term decisions are made, such as determining the number of ORs to open, beds or wards to create, and staff to hire. At the strategic planning level, studies investigate the size (e.g., how many beds and/or rooms to allocate) of EDs [49, 107], wards [213, 226] or both [90, 157, 159, 213, 237] in order to find the number beds that satisfies urgent care demand. At the strategic level, ambulatory services are taken into account in studies that model entire patient pathways [33, 90, 157], and resource capacity is dimensioned across services and wards. For the operating theater, strategic decision making involves the capacity dimensioning of OR capacity among elective and urgent patient types $[68,199,226,253,263]$. Not only the number of needed ORs is determined [199], but also how OR time is assigned to different patients types (e.g., evaluate the use of dedicated emergency ORs) [68, 225, 253].

\section{Tactical planning}

The tactical planning level involves medium term decision making. For the ED, the most studied tactical planning problem is staff-shift scheduling $[1,3,5,23,29,36,39,74,85,92,99,108,118,124,136,144,145,161,188$, $191,210,211,216,220,250,251]$. Shift scheduling deals with the problem of selecting what types of shifts are to be scheduled, and how many and what types of staff are to be allocated to these shifts. As the arrivals of urgent patients at the ED varies throughout the day, it may be beneficial to adapt staff schedules to emergency demand. When scheduling shifts, waiting time of patients, as well as throughput and resource utilization are used as performance indicators. Besides shift scheduling, allocation of resources such as beds or diagnostic equipment is evaluated $[23,29,74,118,144,145,205,208,251]$. When allocating resources, diagnostic resources used at the ED such as ultrasound equipment, or available beds may be dimensioned per patient urgency type. Besides waiting time, utilization of resources, bed occupancy and blocking probabilities (of both patients entering the ED and downstream wards) are used as performance indicators. Alternative patient routing options are also investigated [23, 29, 44, 62, 74, 108, 145, 161, 244], for example, by using fast-tracks. Also, by dedicating staff and resources to the treatment of lower urgency patients it may be possible to find a better distribution of resources and improve overall performance [23, 33, 62, 71, 132, 176, 244]. Patients that enter the ED are triaged, which is done to determine the urgency of a patient, and set the time-frame in which a patient must be seen. This way, extremely urgent patients are seen immediately, while less urgent patients may wait for some minutes or hours. This classification of urgency types also often determines the order in which patients are treated. While most ED studies assume the triage categories and prioritization fixed, some studies investigate alternative admission control policies [12, 13, 211, 212, 255]. [12] compares waiting time, tardiness and length of stay of patients per urgency type under two triage policies. While no overall differences are found, there are differences between urgency 
types, with one triage system performing better for lower urgency patients. [13] investigates the use of a dynamic policy where urgency types may change after arrival. They show it outperforms a static admission control policy for lower urgency patients, gives worse performance for the medium urgency patient types, and yields no discernible difference for the highest urgencies. Another evaluated possibility is the inclusion of treatment complexity in addition to urgency to patient prioritization [212]. Finally, [136] investigates floor layout planning where rooms and reception desks are moved in addition to resource allocation changes.

Tactical operating room planning mostly considers the allocation of capacity to patient groups, or creating Master Surgery Schedules (MSS) in which available OR time is allocated to medical specialties [8, 68, 93, 125, 169, 195, 228, 262]. Taking into account non-elective patients presents additional challenges. Not only are surgery durations stochastic, the arrival of non-elective patients is generally not known in advance, and the question not only involves when and how much capacity to allocate to non-electives, but also how to account for non-electives. To facilitate the timely treatment of non-elective patients, these patients may be treated in dedicated emergency ORs $[8,32,68,199,228,253]$, in regular ORs by interrupting the elective program $[2,68,126,155,253]$, or a combination of both [68, 262]. However the performance of using dedicated ORs (or not) depends on the underlying case settings such as patient mix, volume, and number of available ORs, because conclusions differ on the effectiveness of dedicated ORs $[68,195]$. When urgent patients are treated in the same ORs as elective patients, they are taken into account as by allocating slack $[2,113,126,153$, $207,225,226,247]$ where some part of the ORs is left unused by electives for urgent arrivals. Admission control policies are also evaluated, in which different prioritizations of urgent patients requiring surgery are evaluated [93], as well as staff shift scheduling in which staff shifts are assigned to (emergency) ORs that must quickly be able to respond to emergency arrivals [249] and possibly also treat elective patients [18].

Ward planning studies mostly optimize the allocation of beds to various patient types (e.g., reserve beds for non-electives and electives), as well as dimensioning for different medical specialties $[2,17,18,22,50,83,90,109$, $125,147,150,151,171]$. Complete hospital bed planning is also modeled $[24,109,151,242]$, and when wards are evaluated, elective patients may also be included $[24,103,125,146]$. The focus of the studies is often to maximize bed occupancy, and to minimize waiting time. Also, as wards are seen as facilitators for other departments, the blocking probability (i.e., a failure to accept a patient) is investigated.

When allocating beds to wards, a trade-off between hospital resource utilization and patient service are sought. The coupling effect between ED boarding and ward sizes is also investigated $[142,166,175,246]$, where the size of downstream hospital wards influences the possibility of ED patients to be admitted to wards without waiting (at the ED) for a bed to become available. Similar to ED boarding, the sizing of wards downstream from ORs influences their per- 
formance, as a full ward may result in both elective and non-elective surgeries not being able to start. This interaction is also evaluated through bed allocation $[18,147,226]$. Wards also have a tandem effect, for example as patients flow through acute care and post-acute care units, and the allocation of beds may be optimized to reduce blocking between wards [37, 246]. Discharge policies are also evaluated, which determine when, and under what criteria patients are sent home [51]. The routing of patients is also investigated through the use of process improvements and creation of wards to which only specific non-elective patient types are admitted [71, 97, 169, 193, 194]. Also, the redirection of patients if their intended ward is full is studied [81]. Finally, staff-shift scheduling is also investigated to determine when and how much staff should be assigned to wards based on both elective and non-elective arrivals [81, 258].

Given the nature of hospital wards, where patients spend some time both waiting and undergoing further treatment, these are modeled using queuing models $[45,83,87,166,169,175,217,246,258,260]$, or simulation $[18,22,33,50,51,71,90,97,109,142,150,151,157]$. Complete hospital bed planning is also modeled $[24,109,151,242]$, and when wards are evaluated elective patients may also be included $[24,103,125,146]$.

The most studied tactical ambulatory services planning problem is capacity allocation of resources such as CT scanners, ultrasounds, and outpatient appointment capacity to elective and non-elective patients $[35,79,90,120,123,141,143$, 204]. Given the time dependent uncertain arrivals of non-elective patients, it is not only of interest to determine how much capacity of allocate over patient types, but also when (e.g., [141, 143]). For example, [141] determine blueprint appointment schedules that are most capable to account for non-elective patient arrivals during realization of the schedule. Similar to OR and bed utilization a trade-off is sought between elective patient access time, non-elective waiting time, utilization, and overtime. In addition to capacity allocation, [123] also investigates the effect of parallelization of diagnostic tasks for patients. Changes in patient routing through (re)design of processes is also studied, for example, [215] break up CT diagnostics activities as much as possible such that preparatory steps take place away from the CT scanners, and waiting times decrease.

\section{Operational planning and scheduling}

The operational planning level consists of the operational offline and operational online planning levels. Offline planning concerns the short-term in advance planning of actual patients (i.e., allocating patients a time and resource). The operational online level involves control mechanisms that deal with the monitoring of processes and reacting to unforeseen events. Examples of operational online planning are add-on scheduling of emergencies or triaging. Also, different policies that select the next patient to be treated or diagnosed based on current waiting times and other department characteristics are investigated. These planning decisions are highly connected to the tactical level decisions, given that these decisions direct the way patients are treated and prioritized. For example, the 
tactical decision to allocate a dedicated emergency OR has a large influence on operational decision making.

Several studies investigate the assignment of staff and resources to ED areas in order to reduce patient waiting times and ED blocking [48, 52, 53, 58]. This is done whilst taking into account the current state (i.e., utilization and crowdedness) of the ED, and if necessary available resources are temporarily increased $[47,52]$ or reallocated [148]. Also investigated is the diversion of patients to other departments (e.g., wards) whilst still waiting for examinations and treatment [59]. Such policies may be used once waiting times at the ED exceed certain thresholds. Several (dynamic) admission policies and patient routing policies are also investigated [132, 137, 231]. For example, using fast-track pathways for patients if the ED meets set criteria [132]. Alternative patient pathways and policies assigning non-elective patients to these pathways are also investigated [104]. Finally, patient (time dependent) prioritization rules are also investigated $[111,164,173,232]$, and tools to assist the triage patient categorization that provide further guidelines for patient selection [72].

At the operational level of OR planning, the scheduling of elective patients while taking into account non-elective arrivals is the most studied (53\%). When scheduling patients, a trade-off is sought between operating room (OR) utilization, waiting time, surgery cancellations and overtime. One approach to account for non-elective patients is to use slack planning. Using slack planning, elective patients are only scheduled in a fraction of the available OR. The remaining OR time is then kept empty, to ensure once the schedule is realized there is sufficient time left to treat both non-elective and elective patients and ensure acceptable overtime $[2,113,153-156,201,207,247]$. Additionally, scheduled patients may be postponed and/or rescheduled to account for non-elective patients $[61,63,106]$. Another influence of non-elective patient waiting times is the sequencing of surgeries. By changing the order in which elective surgeries are scheduled, waiting time reductions may be realized as non-elective patients can better fit into the elective schedule $[18,64,77]$. Scheduling elective patients in otherwise dedicated emergency ORs is also done [32], and may result in better utilization of ORs that are otherwise empty awaiting non-electives, as well as access time reductions for electives. Non-elective patient scheduling (policies) are also investigated, with patients of various urgencies that must be scheduled in upcoming ORs [126, 184]. Both mathematical programming $(66 \%)$ and simulation $(43 \%)$ are used, or even combined, to determine patient schedules and evaluate different policies that deal with urgent patients.

Operational planning and scheduling of wards involves the incorporation of post surgery admission to wards in the scheduling of patients $[2,18,61,180]$. The main trade-off concerning wards is on one hand to have high bed occupancies (i.e., utilization), while on the other hand being able to admit all non-elective patients that arrive unexpectedly. Scheduling patients to beds in other wards is also investigated for both elective and non-elective patients [24, 231] or for elective patients while ensuring non-electives may still be admitted [87]. In addition, by 
predicting future bed occupation by elective and non-elective patients, more or fewer electives may be admitted to the wards in order to better utilize wards and offer capacity for non-electives. Discharge strategies are also evaluated that determine when patients are to be discharged if wards are full [45]. When patient discharge strategies are evaluated, the performance indicator often used is the readmission rate of patients. Reallocating beds from less utilized wards to those that are overloaded to minimize crowding is also investigated, which may result in a reduction of bed blocking [109]. Another approach to reduce bed blocking is earlier bed reservation of non-elective patients. By being able to predict when non-elective patients currently at the ED will be ready to be admitted to wards boarding times at the ED may be reduced [206].

At the operational planning level of ambulatory services, the scheduling of patients is studied. Given the blueprints of capacity allocated to patients, both the scheduling of elective patients under uncertain non-elective arrivals is considered [229, 234, 240], as well as scheduling of non-elective patients themselves $[10,16,112,202,229,234,240]$ to specific times in the schedule. The dynamic assignment of patients is also studied. After (elective) patients are assigned to a time in the appointment schedule there may be additional non-elective patient arrivals. In order to improve waiting times, policies that dictate which patient is treated next given based on current patient queues are studied [84, 143, 172]. From the optimal selection policies robust rules of thumb may be derived to apply in practice.

\subsubsection{Urgency classification}

A good patient categorization balances the trade-off between timely access to care and resource utilization appropriately. Besides the distinction between elective and non-elective patients, there may be further categorization between nonelective patients. One reason to categorize non-elective patients is to allow for prioritization based on clinical need and not based on the order of attendance [65]. Such categorization allows that lower priority patients may wait for some time and, may be scheduled later in the planning horizon. This increases planning flexibility, and allows for different planning and scheduling approaches. Also, by categorizing patients in homogeneous groups, a trade-off between patient flow variability, and clinical variability is sought [168]. By identifying more homogeneous patient groups, the clinical variability in, for example, surgery time within the group may be reduced. However, if patient groups become small enough, the variability in patient flow causes too much idle time and waste of resources. Other examples of patient group categorizations are disease type, disease severity, or required resources. In the reviewed studies, patients are categorized in medical categories such as urgent and semi-urgent, which may vary considerably between studies. For example, semi-urgent may both denote patients that must be treated within eight hours [199] or two weeks [263]. To reduce ambiguity and better categorize the literature, Table 2.5 and Table 2.6 list the categorization of patients by target (start of) treatment times underlying the medical catego- 
rizations. Note that "n. s." lists the studies that do not further specify their categorization than general terms such as urgent or semi-urgent.

Table 2.5 Non-elective patient treatment targets, part 1

\begin{tabular}{|c|c|c|c|c|}
\hline target & ED & Wards & OT & Ambulatory \\
\hline Now & 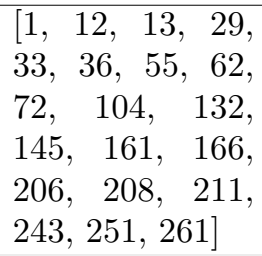 & $\begin{array}{l}{[2,24,104,166,} \\
169,193,206]\end{array}$ & {$[2,63]$} & $\begin{array}{lll}{[84,} & 141, & 172, \\
234] & & \end{array}$ \\
\hline$<10 \mathrm{~m}$ & 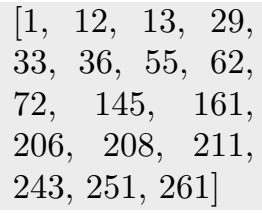 & {$[193,206]$} & & \\
\hline$<15 \mathrm{~m}$ & {$[132,166]$} & [166] & & \\
\hline$<30 \mathrm{~m}$ & $\begin{array}{l}{[55,132, \quad 166,} \\
251]\end{array}$ & [166] & [68] & \\
\hline$<60 \mathrm{~m}$ & 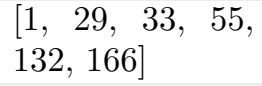 & {$[61,166,193]$} & {$[61,199]$} & \\
\hline$<2 \mathrm{~h}$ & $\begin{array}{l}{\left[\begin{array}{l}1,29, \\
132,166]\end{array}\right.} \\
\end{array}$ & {$[166,193]$} & {$[8,199]$} & \\
\hline$<4 \mathrm{~h}$ & {$[1,29,179]$} & {$[33,193]$} & [199] & \\
\hline$<6 \mathrm{~h}$ & & & {$[8,192]$} & \\
\hline$<8 \mathrm{~h}$ & & & {$[199]$} & \\
\hline$<12 \mathrm{~h}$ & & & [8] & \\
\hline$<18 \mathrm{~h}$ & & & {$[192]$} & \\
\hline$<24 \mathrm{~h}$ & & & {$[8,32,199,201]$} & \\
\hline$<36 \mathrm{~h}$ & & & {$[192]$} & \\
\hline$<2$ days & & & & [202] \\
\hline$<1$-3days & & {$[104]$} & [8] & [240] \\
\hline$<1 \mathrm{w}$ & & & [263] & \\
\hline$<2 \mathrm{w}$ & & & {$[263]$} & \\
\hline
\end{tabular}

\section{Categorization of patients}

In ED studies we find that patients are the most categorized, based on triage models that determine medical urgency. There are various triage systems being used at emergency departments. Of these, the Australian Triage Scale (ATS) [55], Canadian Emergency Department Triage and Acuity Scale (CTAS) [132, 166, 166], Manchester Triage Scale (MTS) [29, 193], and Emergency Severity Index (ESI) $[12,13,36,62,72,145,161,206,206,208,211,243,261]$, and are most seen in practice $[15,65,80,189,254]$. These triage systems all categorize 
Table 2.6 Non-elective patient treatment targets, part 2

\begin{tabular}{|c|c|c|c|c|}
\hline target & ED & Wards & $\mathrm{OT}$ & Ambulatory \\
\hline Day of arrival & & & $\begin{array}{ll}{[153-156,225,} \\
228,262]\end{array}$ & {$[112,229,234]$} \\
\hline n. s. & $\begin{array}{l}3,5,14,19, \\
22,23,39,44, \\
47-49,51-53, \\
58,59,71,74, \\
85,90,92,97- \\
99,107,108, \\
118,121,122, \\
124,127,130, \\
131,136,137, \\
142,144,147, \\
148,152,157, \\
159,163,164, \\
173-176,180, \\
183,188,191, \\
205,209,210, \\
212,213,216, \\
217,220,221, \\
224,231,232, \\
237,244,246, \\
250,255,256, \\
258,259]\end{array}$ & 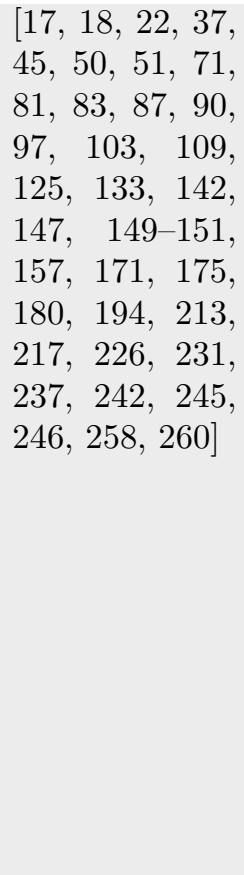 & $\begin{array}{l}{[17,18,64,93,} \\
106,113,125, \\
126,147,171, \\
184,195,207, \\
226,247,249, \\
253]\end{array}$ & $\begin{array}{l}{\left[\begin{array}{l}16,35,79,90, \\
120,123,143, \\
157,174,204, \\
215,260]\end{array}\right.}\end{array}$ \\
\hline
\end{tabular}

patients in one of five levels based on medical characteristics, and list a maximum time to doctor contact based on urgency level. All triage systems have as the highest urgency patients that should be treated immediately, up to a lowest category of patients that must be seen within four hours or less.

We find a change in approaches when the considered problem setting contains patients that must be treated within several days, compared to settings where patients must be treated within 24 hours (i.e., within a day). Over this 24 hour target, studies aim to include non-electives in the scheduling process. Of the six studies that include non-elective patients that may wait longer than one day, one study does not study the scheduling of non-electives. [8] use Monte Carlo simulation of an OR to determine how many ORs to dedicate to non-elective cases. The non-elective patients in their case are treated as soon as possible. The scheduling of non-electives with longer target treatment times is studied for ORs, wards, and ambulatory services. [263] determine the number of surgery slots to offer to (semi-urgent) patients that must be treated within one or two weeks. Additionally, they formulate policies to allocate additional surgery slots to semi-urgent patients by canceling elective surgeries using a Markov Decision Process (MDP). [202] schedule both elective and non-elective radiotherapy patients that must be 
treated within two days using genetic algorithms. They find that by accounting for non-elective patients in the scheduling process reductions in waiting time for all patients are feasible. [240] use simulation and an adaptive scheduling algorithm to schedule elective and non-elective patients for CT-scanners. In their study, non-elective patients must be seen within one, two or three days. In their scheduling approach patients are scheduled FCFS as long as enough time-slots are available for higher urgency patients, and conclude that all patients benefit from an adaptive scheduling approach. [192] schedule non-elective surgeries in an overloaded operating theater (i.e., disaster) setting that must be completed within six, eighteen, or 36 hours. They model their problem using an Integer Linear Program (ILP) formulation, and maximize the number of treated surgical cases. Finally, [104] study admission policies for patients that may wait for one to three days, and are otherwise admitted through the ED. They aim to admit these patients at times such that the canceling of elective admissions and blocking of ED arrivals is balanced.

Ambulatory services studies, with non-elective patients that have a target treatment time of at most a day, focus on resource capacity allocation such that non-electives are treated as soon as possible, and waiting times for elective patients are acceptable. [141] create an appointment schedule blueprint that dictates at what times to schedule non-emergency patients when there are emergency arrivals following time-dependent arrivals rates. Their objective function takes into account the waiting times of patients, idle times of resources, and overtime. [84] also creates an outpatient schedule for ambulatory services that treat both elective and non-elective patients. Additionally, they study dynamic priority rules for admitting patients into service. They formulate their problem as a dynamic program and identify optimal policy properties. [234] study the advance scheduling of elective and non-elective patients (that must be seen on the day of arrival) for MRI diagnostics using dynamic programming. They put forward an algorithm to compute the optimal policy to assign patients to exam days. [172] study the appointment scheduling of CT scanners that provide service to emergency and elective patients. They formulate their problem as a Markov Decision Process (MDP) to decide which appointment requests for diagnostics to accept, and when to schedule the different patient types in order to maximize total revenue.

Studies investigating wards and non-elective patients that must be admitted within a day focus on the efficient allocation of resources [166, 193, 206, 206] and scheduling of patients [24,61]. [193] study a medical assessment unit that takes in urgent medical patients for a short time, and provides a means of rapid assessment and investigation in order to avoid unnecessary admissions. They analyze the allocation of doctors, nurses and beds and use goal programming to optimize the MAU performance and find required resource levels. [166] investigate the coupling effect between ED and inpatient unit ward where patients from the ED are admitted to. They model the patient flow and interaction using queueing theory, and identify the number of required resources at the ED and ward to 
ensure acceptable waiting times. Additionally, they study the use of fast-tracks and its effect of required resources and waiting times. [206] also study the ED bed blocking problem, and evaluate the use of earlier bed reservation policies to reduce patient boarding. The policy takes into account patient specific characteristics to predict probability of admission, and accounts for misclassification (e.g., bed reservation without admission). [24] use mathematical programming to schedule elective and non-elective patients that must be admitted to wards. They take into account patient characteristics such as pathology incompatibilities, and continuity of care, and minimize the waiting time of patients to be admitted. [61] uses mathematical programming to reschedule elective patients if emergency patients arrive. They consider both the operating theater and PACU, and take into account both OR and PACU overtime, the cost of postponing elective surgeries, and turning down emergency patients. Using a genetic algorithm they effectively find solutions for larger instance sizes.

We find that target treatment times are most varied for OT studies, with patients also requiring treatment within six, eight, twelve, and eighteen hours. If patients must be seen within the hour [2, 61, 63, 68, 199], the scheduling of patients $[2,61,63]$ and capacity allocation $[68,199]$ is studied. Given the shortterm treatment times of the non-elective patients, these are treated as soon as possible, and the elective patients are actually scheduled to facilitate timely access for non-electives. This is done through slack planning [2], and rescheduling [61, 63]. The capacity allocation studies investigate the required number of dedicated emergency ORs $[68,199]$, and [68] also compares the use of dedicated emergency ORs to letting patients break into the elective program. There does not seem to be much difference in the problems and approaches studied if non-elective patients may wait longer. $[8,201]$ use simulation to determine the required number of ORs for a setting where patients must be operated on within 24 hours. [32] alternatively studies the possibility of planning elective patients in part of the dedicated ORs, and possibly postponing electives if the OR is needed for nonelective patients. Similar to the setting with more urgent non-elective patients, forms of slack planning are studied where only elective patients are scheduled [153-156, 228, 262]. [192] schedules non-elective patients in an emergency setting using mathematical programming, and maximize the number of patients that can be treated on time. [225] model the operating theater as a markov process, analyze patient flows through the ORs. They find the maximum capacity that may be allocated to electives while still being able to treat non-electives on time. Performance inicators include waiting times, overtime, and utilization

The categorization of patients may be used to prioritize patients. Even though the prioritization of non-elective patients does not necessarily depend on the (medical) urgency of patients, almost all studies note that the prioritization of patients is directly based on the formulated urgency levels, with the highest urgency patients being selected first. Ideally, not only medical urgency, but also other patient (e.g., treatment duration and resource requirements) and system characteristics (e.g., resource availability) are taken into account when prioritiz- 
ing patients. This is also seen in one of the triage systems being used at EDs. The ESI triage system takes into account medical urgency, as well as resource needs and prioritizes patients from must urgent to least resource intensive. Specifically, patients that may wait for some time and are not medically urgent, are prioritized by required resources (multiple, one, or no resources required), with patients requiring multiple resources prioritized first. There are some studies that further investigate the different prioritizations of non-electives.

[10] study different prioritizations of triaging patients. They model a single server clearing system where patients may abandon the ED if not serviced on time. They dynamically determine the (near) optimal order of triage such that the number of abandonments is minimized. [12] also study the triage process at an ED using simulation. They use Multi Attribute Utility Theory (MAUT) to develop a triage algorithm and assist nurses in decision making. They conclude that their approach performs better with regards to minimizing the number of patients that exceed their maximum waiting time. Additionally, it reduces errors in the decision making process. Another study investigating ED triage using simulation is [13], who develop a dynamic grouping and prioritization algorithm that prioritizes patients based on patient and system characteristics. Comparing the triage policies with existing systems (i.e., ESI) they find that length of stay at the $\mathrm{ED}$, and the percentage of patients past their waiting time is reduced. [111] use queuing theory to model patient flow through an ED and formulate policies that determine which patients physicians treat next (i.e., triage new patients or treat 'in process' patients). They find an optimal policy that minimizes congestion of the ED under heavy traffic. Prioritization policies for ambulatory services are also studied. [84] study dynamic priority rules for admitting patients into (diagnostic) service. Based on their optimal policies obtained using a dynamic program they formulate several heuristic scheduling rules that minimize costs. [143] also evaluate different prioritization policies for selecting the next patient to diagnose. They recommend however, to implement a first-come first-serve (FCFS) policy, as the alternative prioritization policies introduce considerable complexity without much performance improvement.

\subsubsection{Methodologies}

In this section we provide an overview of the different methodologies used to model non-elective resource allocation, planning, and scheduling problems. Table 2.7 gives an overview of the used methodologies. There is a clear distinction between used modeling approaches, studied departments and urgency, as the various modeling approaches lend themselves to different problems. If patients must be treated within short time-frames, there is more focus on the proper allocation of resources such that waiting times are minimized. As such, patient flow modeling approaches like simulation and queuing theory are most used for the ED. With these approaches, the patient pathways and processes can be modeled, and changes in resource allocations and processes be evaluated. Simulation is most used for the ED, with 50 studies using simulation as their main methodology to 
model and improve patient flow (e.g., [1, 3, 5, 12]). Queuing theory is used to model both ED and ward patient flows, and only in a few cases used to model the ambulatory services and operating theater [120, 123, 199, 215, 263].

Table 2.7 Classification of papers by used methodology

\begin{tabular}{ll} 
Methodology & References \\
\hline Decision analysis & {$[1,62,72,193,194]$} \\
Dynamic programming & {$[47,59,84,87,104,106,148,156,172,180,245$,} \\
& $247]$ \\
Forecasting & {$[92,127,130,149,163,176,242,256,260]$} \\
Mathematical programming & {$[2,16,24,52,53,58,61,63,64,81,112,125,126$,} \\
& $133,153-155,171,176,192,193,201,207,213$, \\
& $220,221,227-229,245,247,249,262]$ \\
Queueing theory & {$[14,37,45,48,53,58,83,85,87,111,120,121,123$,} \\
& $137,148,164,166,169,175,179,199,201,211$, \\
& $212,215,217,232,243,246,255,258,260,263]$ \\
Stochastic models & {$[84,103,104,143,172,184,225,226,231,232$,} \\
& $237,263]$ \\
Simulation & {$[1,3,5,8,12,13,17-19,22,23,29,32,33,35,36$,} \\
& $39,44,47,50,51,55,58,59,62-64,68,71,73,74$, \\
& $79,90,92,93,97-99,103,104,107-109,111-113$, \\
& $118,121-124,131,132,136,142-145,148,150-$ \\
& $153,155-157,159,161,166,169,171,172,174$, \\
& $180,183,188,191,194,195,199,201,202,204$, \\
& $205,207-213,216,217,220,221,224,225,231$, \\
& $240,244,246,249-251,253,258,259,261]$
\end{tabular}

Even though operating theaters also treat patients who require prompt attention, a large focus is put on the scheduling of elective patients while accounting for non-electives. To this end, mathematical programming is often used (e.g., $[2,63,154,228])$. Additionally, simulation is often used to validate schedules obtained using mathematical programming under different scenarios, and additional stochasticity. The resource capacity allocation problems in which the possible use of, and number of dedicated emergency ORs is decided, is evaluated using simulation (e.g., [68, 225, 253]). The other methodologies are seen more evenly across the various departments.

\subsection{Discussion and conclusion}

The organization of care for non-elective patients is highly complex due to the high stochasticity involved in aspects like arrival time, service time, urgency class, and resource requirements. Non-elective patient pathways typically visit multiple hospital departments, which we have identified and categorized in this chapter, and which are also utilized by elective patients. The majority of papers 
( $82 \%$ ) focus on a single department, with the ED being the most studied. The non-elective patient pathways often however involve multiple departments, and consequently the planning and scheduling of departments affect each other. The literature that models multiple departments with higher detail is scarce, aside from the modeling of entire patient pathways, (e.g., [33, 90, 157]). As the (detailed) modeling of multiple hospital departments may become difficult, an initial step may be to model only key departmental components. The connections between wards, as downstream departments, from the ED and operating theater are studied. However, there are almost no studies that address the relationship between OR planning, and ED patient arrivals. An exception is [147], who predict the hourly bed level of wards based on OR schedules and ED arrivals. Further studies may provide valuable insights into the OR and ED relationship.

With regards to the hierarchical planning levels, the literature that focuses on the strategic planning level is limited compared to the tactical and operational levels. At this level, the required resources of departments are determined, such as the number (and size) of wards to create (e.g., [33]). Most papers study the tactical planning level $(62 \%)$. At the tactical planning level, there are some problems that are studied considerably more. Regarding the ED, the allocation of resources and staff (staff-shift scheduling) is most studied. There is fewer literature on patient routing (e.g., fast-tracks). When fast-tracks are studied, these are often similar in setup, with a staff member reserved to only treat low urgency patients. The question remains, however, whether other patient routings are feasible, and under which circumstances, which is only investigated by [23]. The question of patient routing similarly applies to wards. The allocation of capacity (e.g., how many beds to reserve) is well studied, but the use and evaluation of specialized wards, such as a medical assessment unit, is not. In a sense, such a ward is similar to an ED fast-track and patients that are require some additional diagnostics are admitted to it. It is unclear, however, what patient characteristics, and under what circumstances such specialized wards positively affect patient waiting times, and reduce bed blocking. The tactical operating room planning mostly considers the master surgery schedule problem, taking into account some slack for emergencies. The literature on determining the required amount of slack however is lacking, with studies taking the required slack as given. Also, the master surgery scheduling problem is well studied, and it may be useful to include non-elective patients characteristics at this planning step, by incorporating them into the MSS creation. Also, the policies of using dedicated emergency ORs and breaking into the elective program are both studied. It is unclear, however, under which circumstances the policies perform best, and the full impact of such a policy on performance is unclear. For ambulatory services, the creation of appointment schedules that account for non-elective arrivals is well studied. In such schedules, appointments are kept empty if order to facilitate urgent patient arrivals that must be treated quickly. This problem shows similarities to the operating theater, where elective patients are scheduled, and non-elective patients who require surgery arrive. However there is no research 
done into the postponing of elective surgeries (e.g., scheduling breaks) to facilitate non-elective arrivals.

The operational planning level studies mainly focus on the offline (i.e., in advance) scheduling, such as scheduling staff to ED and wards, or elective patients to the OR. The operational online planning and scheduling however, is more limited, and we identify multiple areas for future research. First, studies that address the changes and adaptation of the (so far) realized patient schedules are scarce, for example by (re)scheduling patients (e.g., [61, 63, 106, 126] waiting time improvements may be achieved. Second, the sequencing of patients may influence waiting times (e.g., [18, 64]), which may be combined with the insertion of breaks into schedules to ensure timely access. A third topic for further research is the scheduling of OR non-electives themselves. Many operating theater papers study a setting where non-elective patients must be treated within the day. This indicates potential efficiency gains may be had by scheduling non-electives, similar to ambulatory settings with patients of similar urgency (e.g., [229, 234]).

Another topic to be addressed in future work is the use of alternative prioritization policies. So far, most studies use a prioritization patients based on medical urgency. While this is desirable for very urgent patients, we find that if multiple urgency categories are modeled, the lower urgency categories are almost always largest. In case of lower urgency patients, these patients must be treated within hours, and it may be interesting to investigate prioritization policies that not only take into account their urgency. For example, prioritization policies that also look at system characteristics such as required resources, resource availability, and expected future system states may be of interest. In the current studies, the changing of priorities is also under-studied. Studies often assume urgencies are fixed, however it is more likely that a patient's urgency changes over time.

In most publications, a generalization of results is lacking, particularly regarding ED modeling, where most studies design a solution methodology for their case study. It is unclear whether the used models may be extended to other settings or cases, and how different solution approaches compare to each other. Development of an instances benchmarking set, similar to those existing for nurse rostering, may be useful to compare and generalize outcomes of ED modeling. In addition, a comprehensive analysis of the characteristics that make up EDs may assist in formulating such a benchmark set.

Finally, we found little evidence of an actual impact of results. Very few studies report about (succesful) implementation of their outcomes.

Concluding, in this review we provide an overview of the non-elective care planning and scheduling literature within hospitals, and categorized the studies according to departmental focus, hierarchical planning level, urgency classification, and chosen methodology. In addition, we identify opportunities for future research using our classification. Our review shows that some areas of non-elective care planning are studied considerably less than others. With this review we hope to stimulate research of under-researched topics, as well as promote the exchange of results between hospital departments studied. 



\section{Simulation and logistics optimization of an integrated emergency post}

\subsection{Introduction}

The delivery of acute care to patients is highly complex and constrained by limited resources. In addition, many of the involved processes are stochastic and there are interactions both within and external to the acute care providers. Consequently, the effects of organizational changes tend to be unpredictable. Therefore, operations research methods, such as computer simulation, are suitable for prospectively evaluating changes such as alternative resource allocations. Indeed, simulation is used often in emergency department modeling [91]. In this chapter we focus on the delivery of acute care outside office hours, when general practitioners practices are closed. In the Netherlands, when people are confronted with an acute care demand outside normal office hours, they choose whether to go to an Emergency Department (ED) or go to a General Practitioners Post (GPP). In the Netherlands, as well as in several other countries, the organization of primary care delivery is shifting towards an increasing integration of triage and advice by phone, as well as larger care provider cooperatives [88]. The self-referring patients -or walk-ins- who arrive at the ED could often have been seen and treated by a general practitioner (GP), at a GPP, with significant cost savings [110].

A new concept is the integrated emergency post (IEP), and similarly to the earlier creation of GPPs, it organizes the provision of after-hours care in a larger cooperative grouping of healthcare providers. The main intention of the IEP is to alleviate ED overcrowding by shifting back primary care demands from the secondary care provider to the primary care provider, while providing the necessary treatment for patients with an acute care demand. The IEP may offer a sustainable solution to ED overcrowding, resulting in both cost savings for the hospital and increased quality of care for the patients. The integration into the IEP gives patients more clarity on where to go with their acute care demand, avoids travel times between the GPP and the ED, and provides more opportunities to treat high urgency patients at the ED. Additionally, as the IEP is a new concept for healthcare providers, this integration of acute healthcare offers new opportunities for efficiency and efficacy gains.

The objective of this study is to prospectively evaluate organizational inter- 


\section{Chapter 3. Simulation and logistics optimization of an integrated emergency post}

ventions and design improvements for an IEP. To this end, we utilize insights from both the screening and the optimization literature as well as domain knowledge of the emergency care environment. A case study is carried out at the IEP in Almelo, where the ED of the hospital ZiekenhuisGroep Twente merged with the GPP Centrale Huisartsen Post Almelo.

The theoretical contribution of our work is twofold. First, we put forward a method to systematically evaluate a large number of interventions simultaneously, using discrete event simulation, to optimize the processes in an acute care chain. Second, we validate and apply this approach to the IEP in Almelo, incorporating patient preferences, and provide insights into the efficient organization of an IEP in general, based on this case study. The practical contribution of our work is the delivery of an sustainable design of the new IEP, taking into account patient preferences and hospital costs, as well as the implementation of a flexible simulation model at the hospital, which includes training of IEP staff in using the model, enabling them to adapt the model when necessary and to evaluate new interventions in the future.

The remainder of this chapter is organized as follows. In Section 3.2, we review the screening and the optimization literature and applications of the reported findings in healthcare. Next, we describe the problem and the simulation model (Section 3.3). In Section 3.4, we present the approach used to define and evaluate interventions. Following this, we give the results from this approach as well as describe the implementation in practice based on the results (Section 3.5). We end this chapter with the conclusions drawn from this study (Section 3.6).

\section{$3.2 \quad$ Literature}

Computer simulation is an often used tool in healthcare studies, with several comprehensive literature reviews detailing its uses $[34,75,91,128,182]$. Within the healthcare domain, much attention has been given to the modeling of EDs, which are evaluated often, compared to other departments [91]. Much of the simulation of EDs is focused on developing and creating valid models. For example, [219] detail a basic approach to creating and using a simulation model, focusing on creating a flexible and easy to use model, as well as stakeholder involvement. Similarly, [129] discuss proven practices used in developing ED simulation models

Following model building and validation, several interventions are usually evaluated. For example, [57] present a detailed simulation model to evaluate different interventions focusing on staff and room availability with the aim to reduce ED waiting times, and [144] evaluate the change in bed availability in an ED. Simulation is often proposed to evaluate various interventions in an ED. In a literature review on the use of simulation to investigate overcrowding of EDs, [200] divide the different evaluated interventions over resource related, process related, and environmental related scenario testing. Most of the reported articles focus on the reduction of waiting times for patients [200].

Our goal is to prospectively evaluate many different interventions, using a 
simulation model, and to find an optimal process design of the IEP. Related methodologies to support this are factor screening and simulation optimization. Factor screening is used to screen for influential factors, and aims to reduce model complexity and computation time, while still achieving good outcomes [134, 138]. Simulation optimization attempts to find the combination of controllable factor settings that lead to the best outcome [9]. However, it must also deal with noise, as simulation outcomes are approximations of true performance indicators.

A straightforward screening method is a full factorial or $2 \mathrm{k}$ design, such that both main effects as well as interactions may be evaluated [160]. A downside of this method is that, as the number of factors increase, many runs are required. To overcome this, fractional factorial designs may be used, which require fewer runs, while both main and some interaction effects can still be estimated [160]. However, care should be taken not to alias potentially important effects [187]. Other screening approaches for a large number of factors are two-stage group screening [119, 177, 233], sequential bifurcation [25, 46, 257], iterated fractional factorial designs (IFFD) and supersaturated designs (SSD). We see that among the different screening techniques, many are specific in regard to the assumptions made, the maximum number of factors, applicability regarding qualitative and (continuous) quantitative factors, and the use of information already known before screening, such as the signs of interaction.

Different approaches to simulation optimization are ranking \& selection $[28,78]$, response surface methodology and gradient-based procedures [78], random search [7], and sample path optimization and metaheuristics [9]. An example of simulation optimization applied to an ED is given by [3], who determine the number of staff required to optimize patient throughput and waiting times. Similar to the screening methods, the application of optimization techniques relies on characteristics of the underlying simulation model. Depending on input variables and constraints, certain optimization methods are more applicable than others. For more details we refer to [6] and [20]. An additional challenge with optimization is that the methods may propose a solution which requires many organizational changes within the ED, which might prevent a successful implementation.

Much of the literature on simulation in healthcare focuses on model construction and validation, with less emphasis on defining and evaluating possible interventions and their interaction effects. This has motivated us (i) to propose a way to evaluate and optimize over a large number of interventions using a discrete event simulation model and (ii) to consider the optimal organization of a new innovative concept in healthcare, i.e., the IEP.

\subsection{Problem description}

In this section, we give a general description of an IEP and describe the implemented IEP in Almelo. Following this, we briefly address the simulation model that is used to evaluate the IEP and the way in which we incorporate patient 


\section{Chapter 3. Simulation and logistics optimization of an integrated emergency post}

and community preferences in formulating performance indicators. This research is part of a research project studying the optimal process design of an IEP [56]. To this end, a simulation model of the IEP is constructed [181], which we update and expand upon in order to model and systematically evaluate all interventions. For a complete description of this simulation model we refer to [181].

\subsubsection{Integrated Emergency Post design}

In an IEP, the GPP and ED work together to provide acute care to patients outside office hours. The largest change with the introduction of the IEP is that self-referring patients, or walk-ins, are now first seen at the GPP, instead of possibly going to the ED. There are several ways in which patients may enter the IEP: by calling the IEP, going to the IEP as a self-referral, and by being referred to the IEP by an external care provider. When a patient calls the IEP, a telephonic triage takes place, and depending on the urgency of care demand, the patient either gets a consultation at the IEP, a doctor visits the patient, the patient is referred straight to the ED (by ambulance), or the patient receives medical advice by phone. Self-referring patients first undergo physical triage by a GP assistant, after which they are either sent home with medical advice, scheduled for a GP consultation or referred to the ED. Finally, external referrals are sent directly to the ED.

Patients that receive an appointment via telephone or self-refer and enter the IEP, are first seen at the GPP. In most cases, this treatment is sufficient after which a patient goes home. Possibly, the patient may require an X-ray, after which, depending on the results, a patient goes home, or in case of a fracture, is referred to the ED. Similarly, other patients that cannot be treated at the GPP, or require additional treatment, are further referred to the ED. Patients that enter the ED are first triaged again, as the triage system differs from the one used at the GPP, and then the patient history is registered by an ED nurse or physician assistant. Afterwards, a patient might undergo multiple diagnostic tests and receives treatment. After all treatment is finalized, the patient leaves the system and continues his care path outside the ED or goes home. Additionally, some patients require multiple diagnostic tests with treatment after each test. Figure 3.1 shows a conceptual view of the care pathways defined of the IEP in Almelo.

As the IEP is a new concept, many organizational changes may improve the IEPs efficiency, e.g., by pooling resources between the GPP and the ED, which can be prospectively evaluated using simulation. The IEP in Almelo has been modeled and validated in a detailed simulation model.

The simulation model is characterized by patients, resources, and processes. Patients move through the IEP and require resources in the form of healthcare professionals, diagnostic test equipment, and beds/rooms. Patients and resources are characterized by various attributes, such as urgency, diagnostics needed, availability, and capacity. The processes define the care path the sequence of steps that a patient must undergo. Within the simulation model, the arrival of patients, as well as the occurrence of treatments and diagnostic tests are modeled 
Figure 3.1 Patient flow and processes at the IEP Almelo [181]

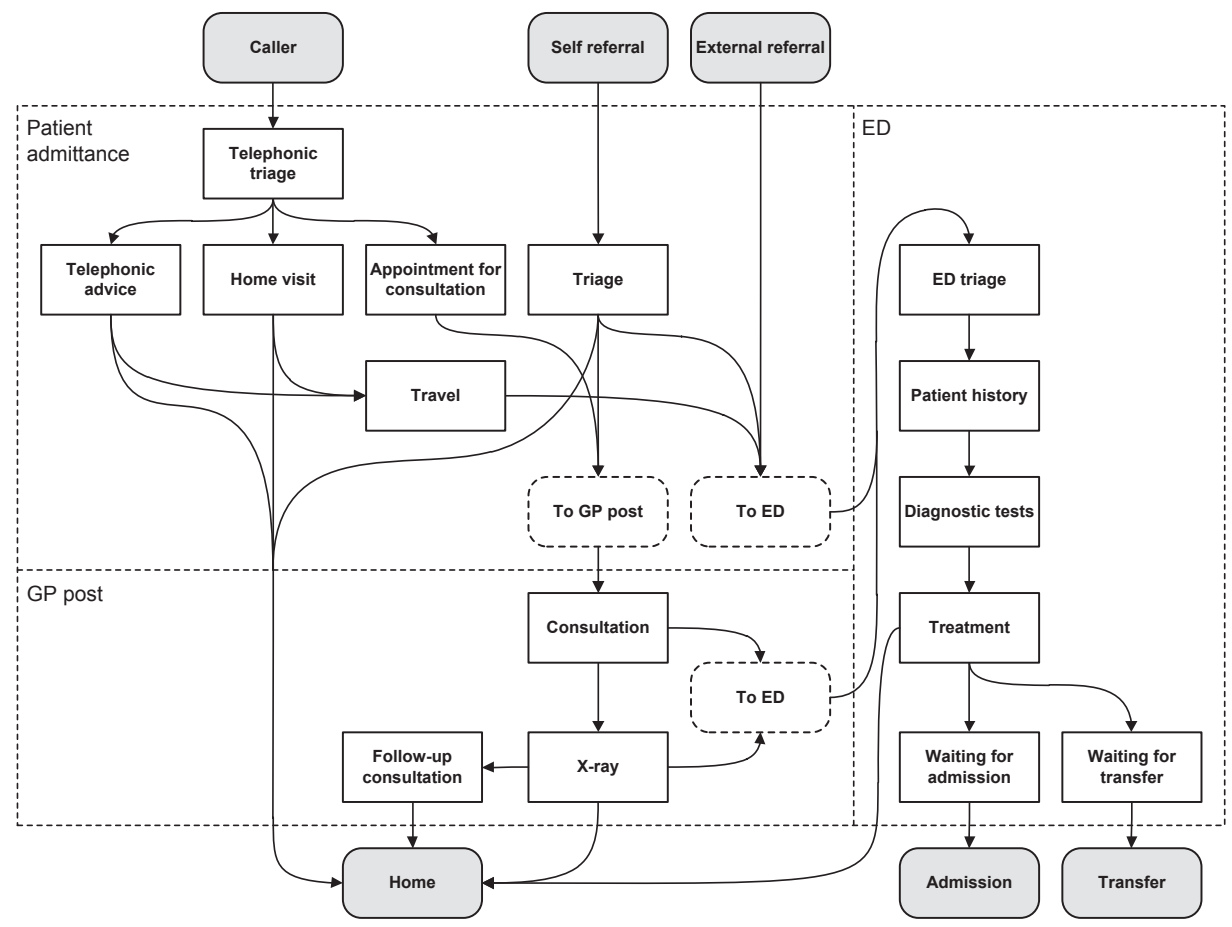

using probability distributions based on four year of historical data, as well as on expert opinion.

The inter-arrival times between patients entering the IEP are described using a stochastic arrival process, depending on the time of day, day of the week, and week of the year, and based on multiple years of historical data. The resources in the model are distinguished as staff, equipment and rooms. The following staff is included: GP, GP assistant, triage assistant, ED nurse, nurse practitioner, physician assistant, medical resident and surgical resident. In addition, medical specialists and diagnostic nurses are included as external staff, who may be called to the ED when needed. Regarding equipment and rooms, we include one triage room, six GP rooms, eight ED rooms, a CT room, two plaster rooms, and two $\mathrm{x}$-ray rooms. In addition there is portable ECG and ultrasound equipment which are used in combination with an ED room. For more details on the components and the construction of the simulation model, we refer to [181].

\subsubsection{Modeling for sustainability}

Organizations place an increasing focus not only on cost related performance indicators, but also on social and environmental factors. These factors, and especially the social factors, are crucially important to healthcare organizations. This research is part of a research project studying the optimal process design of an IEP, 


\section{Chapter 3. Simulation and logistics optimization of an integrated emergency post}

such that patients are seen by the right care provider, without unnecessary delays, while accounting for patient preferences [56]. This research scope accounts not only for production measures, but for social and environmental measures as well. In addition to a simulation study and advice aimed at improvement of the current situation, the simulation model is designed flexibly, to enable healthcare professionals (policy workers) to adapt the model to the changing environment and to evaluate further improvements in the future.

To account for patient preferences, and to construct social and equitable performance indicators for this study, [76] conducted a patient and community preference analysis using best-worst scaling. These outcomes showed that patients primarily value (less) waiting times, followed by access to care provider (selfreferral over appointment based), and type of care provider (physician over nurse practitioner). These indicators are incorporated in the simulation model, which is now used by the hospital. To keep our presentation concise, we focus mainly on the key performance indicator, i.e., the patient length of stay. To optimize the IEP, we aim to evaluate the effects of all potential interventions, and also account for the interaction that can take place between interventions. However, as the number of potential interventions increases, an evaluation of all combinations becomes intractable. Therefore we want to optimize the IEP without evaluating every intervention alternative. The next section formulates the approach to first identify potentially effective interventions and then further evaluate these interventions.

\subsection{Approach}

In this section, we formulate the approach used to identify and evaluate potential organizational interventions. We first formulate and categorize the interventions into several categories and eliminate ineffective interventions. Following this, we evaluate the remaining interventions and combine these into several combinations.

Given the simulation runtime, a full factorial design on many interventions quickly becomes intractable. In addition, some interventions can have many variations, such as changing staff numbers, creating many potential staffing schedules. We first want to assess for which of these interventions further evaluation seems promising. To do this, evaluating interventions in groups may reduce the number of possible alternatives. Furthermore, it is unlikely that the management of the IEP will accept drastic organizational changes in which many interventions are being combined. A structured approach in which we identify only a few, say three, most influential interventions per group, will result in a smaller set of interventions that increase the effectiveness of the IEP without drastically changing its organization. Therefore, we use the approach given below.

- List interventions

- Categorize interventions based on type and specificity 
Evaluate (interaction) effects

Select the most effective interventions per group

- Formulate intervention sets

- Compare intervention sets

Evaluate absolute intervention outcomes

Scenario analysis

Similar to the overview made by [200] who categorize interventions evaluated in healthcare simulation, and make the distinction between process, resource, and environmental (e.g., increasing patient arrivals) changes. Following this, we are going to group interventions into process and resource changes. In addition, we further divide the resource interventions into subgroups consisting of interventions of similar nature, namely staff, diagnostics, allocation, and a rest group.

Each rest group intervention is a combination of several smaller interventions, which are not incorporated in the other categories. For example, an earlier or later start of the main shifts gives insights into the fit between patient arrivals and number of staff, which is a combination of several varying staffing levels and starting times. The environmental changes are similar to simulations carried out in a scenario analysis, evaluating the effect future demographic changes may have.

Based on the patient and community preferences we compare interventions using the patient length of stay (LOS) as key performance indicator, which incorporates both treatment and waiting times. Some interventions target the ED or GPP specifically, so we split the LOS in GPP LOS and ED LOS. However, some interventions may target specific patient groups (e.g., high urgency patients). For these interventions, the LOS indicators are rough estimates, as patients excluded from the intervention are included in the measurement.

New interventions are formulated from the groups, combining the effective interventions. This is done not only based on simulation outcomes, but also based on expert opinion about the feasibility of the interventions (e.g., staff availability and costs). Ideally, an equitable distribution of healthcare places more emphasis of those patients that need more care (i.e., high urgency patients), therefore the intervention set alternatives are compared by evaluating LOS for both high and low urgency patients individually.

\subsection{Results}

In this section, we conduct the approach described before and evaluate the (interaction) effects within each of the intervention groups. Following this, we select the interventions that show significant improvements, and use these to formulate intervention sets, which we then compare using the simulation model. Finally, 


\section{Chapter 3. Simulation and logistics optimization of an integrated emergency post}

a detailed evaluation and scenario analysis is carried out on the most promising intervention sets.

\subsubsection{List interventions}

To come up with interventions to evaluate, we use both simulation literature and interviews with stakeholder from the GPP and ED to define as many interventions that seem feasible to simulate and implement. In total 24 interventions are defined. For example, as an intervention hospital stakeholders noted the possibility to utilize a single triage system, such that patients that are referred from the GPP to the ED no longer have to be triaged a second time. Another example is the allocation of staff to patients. Currently, if a patient arrives, ED specialists or residents are assigned first to that patient, and if they are not available, a physician assistant is assigned. As an intervention this prioritization is reversed, so that lower urgency patients are treated first by a physician assistant, keeping ED specialists and residents available for higher urgency patients that may arrive. An overview of all defined interventions is given in Table 3.1, the numbers in parenthesis indicate the intervention number.

Table 3.1 Identified and categorized interventions

\begin{tabular}{|c|c|c|}
\hline Category & Group & Intervention \\
\hline \multirow{5}{*}{ Process } & & Use a single triage system (1) \\
\hline & & Change triage protocol to let ED nurse order diagnostics (2) \\
\hline & & Give priority only to high urgency patients (3) \\
\hline & & Assign physician assistants to patients before physicians (4) \\
\hline & & Initiate request of hospital admission earlier $(5)$ \\
\hline \multirow{6}{*}{ Resource } & \multirow{6}{*}{ Staff } & Vary the numer of ED nurses (6) \\
\hline & & Vary the numer of surgical residents (7) \\
\hline & & Vary the numer of internal medicine residents $(8)$ \\
\hline & & Vary the numer of psysician assistants (9) \\
\hline & & Vary the numer of general practitioners (10) \\
\hline & & Vary the numer of ED specialists (11) \\
\hline \multirow{4}{*}{ Resource } & \multirow{4}{*}{ Diagnostics } & Vary the numer of X-ray rooms (12) \\
\hline & & Vary the numer of CT scan rooms (13) \\
\hline & & Vary the numer of ECG equipment (14) \\
\hline & & Vary the numer of Ultrasound equipment (15) \\
\hline \multirow{4}{*}{ Resource } & \multirow{4}{*}{ Allocation } & Treat (low urgency) ED patients in GPP rooms (16) \\
\hline & & Let physician assistants treat both ED and GPP patients (17) \\
\hline & & Let ED nurse treat GPP patients (18) \\
\hline & & Let ED nurse specialists/residents treat GPP patients (19) \\
\hline \multirow{5}{*}{ Resource } & \multirow{5}{*}{ Rest } & Use medical specialists at IEP at all times (20) \\
\hline & & Use future hospital roster (21) \\
\hline & & Replace internal medicine resident with ED specialist (22) \\
\hline & & Earlier main shift (GP/GP assistant/ED nurse) (23) \\
\hline & & Later main shift (GP/GP assistant/ED nurse) (24) \\
\hline
\end{tabular}




\subsubsection{Categorize interventions}

Following the listing of interventions, a categorization is made based on the type of intervention as well as on the resulting number of interventions per category. Table 3.1 lists the division of interventions over the possible categories. The interventions in the category process and in the group allocation are all binary (on/off), and the diagnostics and staff changes may have more alternatives, by adding or subtracting more than one resource. In addition, staffing changes can also be temporal in nature by changing the starting times. To evaluate the potential of staff interventions, we add an additional staff member during the busiest hours of the IEP. Based on these outcomes, we can further specify feasible staffing alternatives in the next step. Similarly for diagnostics, an additional diagnostics machine is added in the simulation model. The rest category consists of larger organizational interventions, which are a combination of smaller interventions.

For the first four groups, the insights into the effects as well as interaction between interventions are evaluated using a full factorial design, and used to define the (up to) three most promising interventions. As the interventions in the rest group are not combinable staffing alternatives, these are evaluated individually.

During the categorical evaluation, we set the run length such that the specified precision of the most variable intervention has at most a relative error of $5 \%$ with a confidence level of $95 \%$ [160]. Using this method, the simulation run length is 32 weeks per experiment for the process, staff, and diagnostics designs; 48 weeks for the pooling and allocation design; and 31 weeks for the roster design. Table 3.2 lists the number of experiments per factorial design that are evaluated initially, as well as the number of simulation runs carried out per experiment. In total, 133 different intervention combinations are evaluated. This results in a total of 4507 runs.

Table 3.2 Number of experiments and interventions per intervention category

\begin{tabular}{l|c|c|c}
\hline Experimental design & interventions & experiments & runs (exp x repl) \\
\hline Process interventions & 5 & $32\left(=2^{5}\right)$ & $1024(32 \times 32)$ \\
Staff interventions & 6 & $64\left(=2^{6}\right)$ & $2048(64 \times 32)$ \\
Diagnostics & 4 & $16\left(=2^{4}\right)$ & $512(16 \times 32)$ \\
Pooling and allocation & 4 & $16\left(=2^{4}\right)$ & $768(16 \times 48)$ \\
Roster interventions & 5 & $5\left(=5^{1}\right)$ & $155(5 \times 31)$ \\
\hline Total & $\mathbf{2 4}$ & $\mathbf{1 3 3}$ & $\mathbf{4 5 0 7}$
\end{tabular}

\subsubsection{Category outcomes}

Of the 19 interventions evaluated with full factorial designs, twelve are found to have a significant main effect within their respective groups, with an additional five significant interaction effects. Figure 3.2 lists all the main and two-way interactions that are found to be significant $(\alpha=0.05)$.

Several process changes show an improvement at the ED. Both a single triage system (1), as well as a direct ordering of more diagnostic tests (2) reduce the 


\section{Chapter 3. Simulation and logistics optimization of an integrated emergency post}

Figure 3.2 Significant ED and GPP main and two-way interaction effect confidence intervals $(\alpha=0.05)$

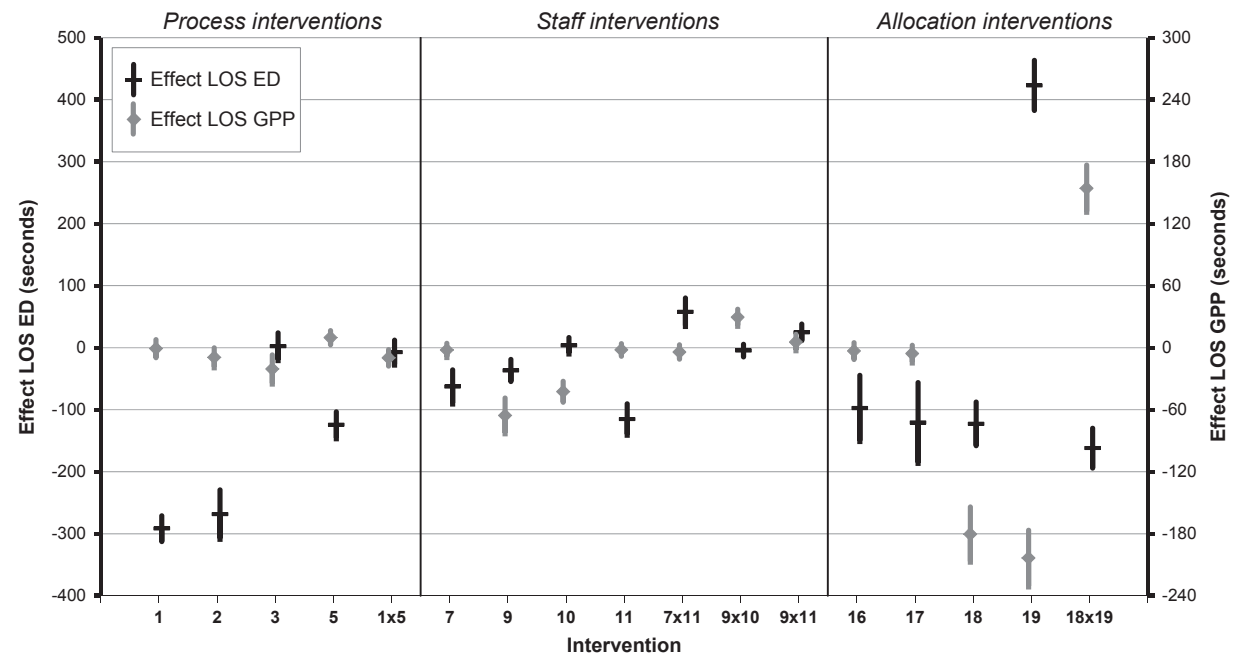

ED LOS by approximately 300 seconds (5\%). Furthermore, the direct admission requests of specific patient groups reduce ED LOS by approximately 120 seconds (2\%). Reprioritizing patients (3) reduces average GPP LOS by 20 seconds (1.3\%); however, from results not shown here, we see that the LOS decreases by $10 \%$ for low urgency (U4) patients, while LOS increases by $4 \%$ and $18 \%$ for U2 and U3 patients respectively.

The biggest influence on reducing the GPP LOS is achieved by adding a physician assistant (9), followed by adding a general practitioner (10). In addition, we see that the two-way interaction effect between these staff types is positive, interpreting this, the combination of these staff types has a diminished effect on the reduction of the length of stay. For the ED, we see that the biggest influence is achieved by adding a ED specialist (11), followed by adding a surgical resident (7) and adding a physician assistant (9). By adding these staff types, the average length of stay is reduced by 115, 60 and 36 seconds respectively.

Looking at the interaction effects on the GPP and ED length of stay, we see that the biggest reduction on GPP LOS is achieved by allowing ED staff to treat GPP patients (18 and 19). Additionally, there is an interaction effect between these interventions, where the interventions have a dampening effect on each other. Similar to the staff interventions, once multiple extra staff members start treating GPP patients, the effect decreases, as the bottleneck shifts from staff availability to another resource type, or that there are no longer enough patients entering the IEP to see a significant reduction in length of stay. The roster alternatives show a large reduction in ED LOS when medical specialists instead of residents are scheduled at the IEP, instead of traveling to the IEP when requested for consultation, resulting in a reduction of over 10 minutes (10\%). However, this would also be a costly intervention, as specialist salaries 
are considerably higher than those of the residents.

Based on these outcomes, combined with insights on associated costs and feasibility, we identify the following effective interventions.

- Use a single triage system (1)

- Change triage protocol to let ED nurse order diagnostics (2)

- Initiate request of hospital admission earlier (5)

- Vary the number of surgical residents (7)

- Vary the number of physician assistants (9)

- Vary the number of ED specialists (11)

- Treat (low urgency) ED patients in GPP rooms (16)

- Let physician assistants treat both ED and GPP patients (17)

\subsubsection{Formulate intervention sets}

As the variations of staff allow for many different schedules, we further specify interventions which are not of a binary nature, and formulate several cost equivalent alternatives. These alternatives combined with the binary process and pooling interventions result in the interventions shown below.

- Use a single triage system

- Change triage protocol to let ED nurse order diagnostic

- Initiate request of hospital admission earlier

- Treat (low urgency) ED patients in GPP rooms

- Let physician assistants treat both ED and GPP patients

- Roster alternatives

Replace surgical resident with ED specialist and add a physician assistant during the weekends busy hours (similar to intervention 21, future hospital staffing schedule)

Schedule two physician assistants during the Saturday and Sunday busy hours instead of a general practitioner

Schedule a surgical and internal medicine resident instead of the ED specialist during the Saturday and Sunday busy hours

Schedule a physician assistant instead of an ED nurse during the Saturday and Sunday busy hours

Schedule a physician assistant instead of an ED nurse during the first opening hours of the IEP (5pm-8pm) 


\section{Chapter 3. Simulation and logistics optimization of an integrated emergency post}

The different process and pooling interventions can be combined with each other to form different intervention sets. However, none of the five process and pooling interventions showed interaction within their respective groups, and it seems likely that there will be little interaction between these interventions. Therefore, we expect that a combination of these interventions, together with a roster alternative will show the greatest LOS reduction for the IEP. To evaluate the effect of the roster alternatives, we combine each of them with the selected process and pooling interventions as shown in Table 3.3. Note that intervention 6 e only affects the weekday evening schedule, instead of the weekend days.

Table 3.3 Intervention sets

\begin{tabular}{lllllllllll}
\hline Set \Intervention & 1 & 2 & 3 & 4 & 5 & $6 \mathrm{a}$ & $6 \mathrm{~b}$ & $6 \mathrm{c}$ & $6 \mathrm{~d}$ & $6 \mathrm{e}$ \\
\hline Set 1 & $\mathrm{x}$ & $\mathrm{x}$ & $\mathrm{x}$ & $\mathrm{x}$ & $\mathrm{x}$ & $\mathrm{x}$ & & & & \\
Set 2 & $\mathrm{x}$ & $\mathrm{x}$ & $\mathrm{x}$ & $\mathrm{x}$ & $\mathrm{x}$ & & $\mathrm{x}$ & & & \\
Set 3 & $\mathrm{x}$ & $\mathrm{x}$ & $\mathrm{x}$ & $\mathrm{x}$ & $\mathrm{x}$ & & & $\mathrm{x}$ & & \\
Set 4 & $\mathrm{x}$ & $\mathrm{x}$ & $\mathrm{x}$ & $\mathrm{x}$ & $\mathrm{x}$ & & & & $\mathrm{x}$ & \\
Set 5 & $\mathrm{x}$ & $\mathrm{x}$ & $\mathrm{x}$ & $\mathrm{x}$ & $\mathrm{x}$ & & & & & $\mathrm{x}$ \\
\hline
\end{tabular}

\subsubsection{Compare intervention sets}

To evaluate the effect of the intervention sets, we make a distinction between length of stay at the ED and GPP, as well as in length of stay during weekend and weekdays. Additionally, we look at the length of stay for high (U1 \& U2 at the GPP, and red \& orange at the ED) and low (U3 \& U4 at the GPP, and yellow-blue at the ED) urgency patients. Figure 3.3 shows the effect of the various intervention sets with $95 \%$ confidence intervals. We set the run length such of the experiments such that the specified precision of the most variable intervention and LOS measurement has at most a relative error of $5 \%$ with a confidence level of $95 \%$ [160]. This results in a run length of 222 weeks per experiment.

All intervention sets improve the ED LOS compared to the original situation, as not only the procedural interventions are implemented, there is also an additional staff member working during the busy hours. The highest decrease is shown when two residents are scheduled instead of an ED specialist (set 3). However, the differences in LOS between intervention sets are small.

Similarly, we see a decrease in GPP LOS. However, the effects on high urgency patients is remarkably lower, with the confidence intervals overlapping with those of the current situation. Replacing a GP with two physician assistants (set 2) has the greatest effect on lowering the low urgency patient LOS. However, the differences between combinations are all minor. The differences in ED LOS between the best and worst intervention set differs approximately 100 seconds, and at the GPP the differences are around 60 seconds.

As no alternative intervention set decreases LOS significantly more than any other intervention set, the most promising weekend alternative seems to be $6 \mathrm{a}$ (set 1), given that this is a roster alternative preferred by the IEP stakeholders. 
Figure 3.3 Average LOS outcome confidence intervals (95\%) per intervention set

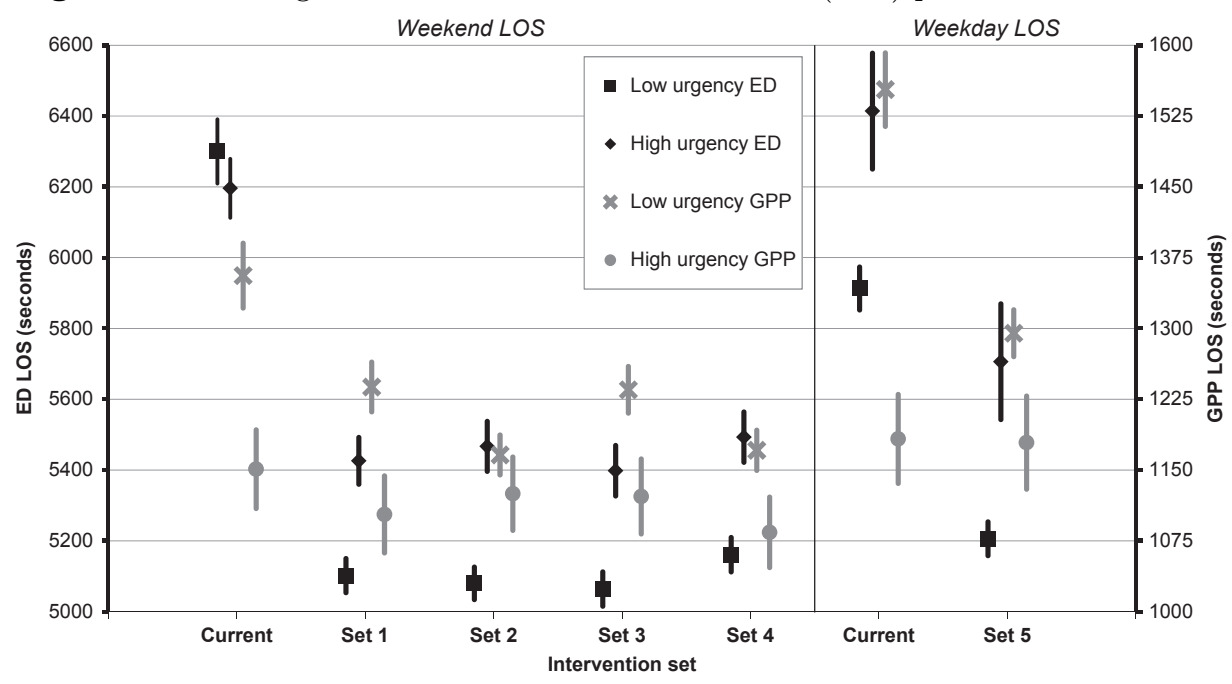

In addition, the weekday replacement of an ED nurse with a physician assistant (6e) that treats both GPP and ED patients shows a decrease for all low urgency LOS patients, making this a promising alternative as well.

We simulate the interventions from intervention set $6 \mathrm{a}$ and $6 \mathrm{e}$ individually to assess the absolute effects of each individual intervention on the IEP, and compare them to each other. Table 3.4 shows the length of stay for both GPP and ED over both type of day (weekend or weekday) as well as high or low urgency patients. The bolded outcomes are found to be significant $(\alpha=0.05)$.

Overall, all selected interventions show a significant improvement over the current situation. Of these, the roster alternatives show the greatest effect on GPP LOS, and process interventions on ED LOS. In addition, the pooling of resources, such as staff, shows that both the GPP and ED can benefit, while the overall staffing costs remain virtually unchanged. In addition, the interventions have no negative effects on any subset of patients. This is especially important for the interventions that target the low urgency patient groups, as an (overall) decrease in LOS may still allow for an unequal distribution of care over patient groups.

With a sensitivity analysis we evaluate the effects of potential environmental changes. By varying the number of patient arrivals, as well as the urgency of patients, the LOS is measured for both the current organization of the IEP, and all interventions from Figure 3.4 combined. The sensitivity analysis results show that with the selected interventions, both ED and GPP are able to treat both more patient arrivals and more acute patients. For example, When patient arrivals increase, the difference in average GPP LOS between selected interventions and the current organization of the IEP increases. At a $20 \%$ patient increase, the average GPP LOS increases by approximately $65 \%$ in the current situation, and $40 \%$ with the selected interventions. At a $50 \%$ patient increase, the average GPP 


\section{Chapter 3. Simulation and logistics optimization of an integrated}

emergency post

Table 3.4 Absolute outcomes (in seconds) per intervention

\begin{tabular}{|c|c|c|c|c|}
\hline $\begin{array}{c}\text { GPP } \\
\text { performance }\end{array}$ & $\begin{array}{l}\text { Weekday } \\
\text { high urgency }\end{array}$ & $\begin{array}{l}\text { Weekday } \\
\text { low urgency }\end{array}$ & $\begin{array}{l}\text { Weekend } \\
\text { high urgency }\end{array}$ & $\begin{array}{l}\text { Weekend } \\
\text { low urgency }\end{array}$ \\
\hline Current & 1186 & 1557 & 1151 & 1356 \\
\hline 1 & $+4(+0.4 \%)$ & $+9(+0.6 \%)$ & $-28(-2.5 \%)$ & $-16(-1.2 \%)$ \\
\hline 2 & $+11(+0.9 \%)$ & $+14(+0.9 \%)$ & $-32(-2.8 \%)$ & $-5(-0.4 \%)$ \\
\hline 3 & $+33(+2.8 \%)$ & $+19(+1.2 \%)$ & $-28(-2.5 \%)$ & $-14(-1 \%)$ \\
\hline 4 & $+29(+2.4 \%)$ & $+3(+0.2 \%)$ & $-8(0.7 \%)$ & $+4(+0.3 \%)$ \\
\hline 5 & $+8(0.7 \%)$ & $-38(-2.4 \%)$ & $-32(-2.8 \%)$ & $+12(+0.9 \%)$ \\
\hline $6 \mathrm{a}$ & $+19(+1.6 \%)$ & $-34(-2.2 \%)$ & $-21(-1.8 \%)$ & $-122(-9 \%)$ \\
\hline $6 \mathrm{e}$ & $-8(-0.7 \%)$ & $-274(-17.6 \%)$ & $-7(-0.6 \%)$ & $-7(-0.5 \%)$ \\
\hline ED & Weekday & Weekday & Weekend & Weekend \\
\hline performance & high urgency & low urgency & high urgency & low urgency \\
\hline Current & 6421 & 5901 & 6201 & 6299 \\
\hline 1 & $-193(-3 \%)$ & $-287(-4.9 \%)$ & $-183(-2.9 \%)$ & $-349(-5.5 \%)$ \\
\hline 2 & $-366(-5.7 \%)$ & $-203(-3.4 \%)$ & $-381(-6.1 \%)$ & $-290(-4.6 \%)$ \\
\hline 3 & $-215(-3.3 \%)$ & $-126(-2.1 \%)$ & $-141(-2.3 \%)$ & $-109(-1.7 \%)$ \\
\hline 4 & $+36(+0.6 \%)$ & $-119(-2 \%)$ & $-18(0.3 \%)$ & $-108(-1.7 \%)$ \\
\hline 5 & $+5(+0.1 \%)$ & $-15(-0.3 \%)$ & $-71(-1.2 \%)$ & $-236(-3.7 \%)$ \\
\hline $6 \mathrm{a}$ & $+17(+0.3 \%)$ & $-46(-0.8 \%)$ & $-238(-3.8 \%)$ & $-476(-7.5 \%)$ \\
\hline $6 e$ & $+26(+0.4 \%)$ & $-11(-.2 \%)$ & $-28(0.4 \%)$ & $-7(-0.1 \%)$ \\
\hline
\end{tabular}

LOS with the selected interventions increases with $80 \%$, while the GPP LOS in the current situation increases with $250 \%$, showing that an optimized IEP is better equipped to treat an increasing number of patients.

\subsubsection{Implementation}

Based on the simulation study outcomes, the effect of adding a physician assistant that treats both GPP and ED patients during the starting hours of the IEP has been trialed in a pilot study. During a three week measurement period (January 2013), every week day from 5pm until 8pm, a physician assistant consulted and treated both low urgency GPP patients, as well as low urgency ED patients. This pilot study showed a positive effect for GPP patients, reducing the average waiting time, while having no effect of ED patient LOS. These outcomes are similar in the simulation model, where average GPP LOS reduced by 120 seconds without significant effect on the ED LOS. From this we conclude that the simulation model is a valid representation of the actual situation.

\subsubsection{Sustainability}

By using community and patient preferences in the key performance indicators, we evaluated both economic and social effects of the interventions. From our results, we see that all patients benefit from using the IEP, and those patients that need the most care, receive the most benefit (i.e., ED patients). An additional 
side effect of the integration into an IEP is environmental in nature. With the repositioning of care providers such that they are placed at a single location, both travel time and costs that patients would have incurred when traveling between the GPP and the ED are removed.

The end product of this research is not only an advice to the healthcare providers on the optimal process design for the IEP in the current situation, but to deliver a reusable simulation tool and approach that healthcare professionals may use themselves to evaluate potential interventions that arise in future situations. To this end, simulation tutorials have been given to IEP stakeholders, including physicians, ED managers, and the GPP director. During these tutorials, simple healthcare processes have been modeled to gain understanding and acceptance of simulation modeling, and the participants could evaluate various interventions by themselves. One hospital employee has been assigned to keep the simulation model up to date, and use it to further help and evaluate the IEP in continuous and sustainable process improvements.

\subsection{Conclusion}

We used a systematic approach in defining and evaluating many organizational interventions for an Integrated Emergency Post (IEP). We did this by first grouping interventions and selecting the most effective interventions per group. Following this, we formulated intervention sets which were compared and further evaluated. Using this approach, we evaluated both the effects of potential interventions, as well as the interaction between these interventions. This enabled us to compare and evaluate many changes while keeping the required simulation time feasible. The use of the simulation model, as well as the structured approach was essential to evaluate the IEP in Almelo, without intervening in the actual processes of the IEP.

We identified various interventions, divided by process interventions, staffing interventions, and resource allocation interventions, showing a reduction in patient LOS at both the GPP and ED. Process and allocation interventions show a decrease of $2-6 \%$ in ED LOS, and staffing alternatives reduce GPP and ED LOS with $3-17 \%$, depending on the patient group. The IEP offers a sustainable solution to the problem of ED overcrowding, where all stakeholders benefit: better care is provided to patients by increasing clarity (i.e., for patients to decide where to go) and effectiveness, at reduced costs. By accounting for patient and community preferences, various interventions have been identified resulting in positive effects on both social and environmental factors without causing drastic changes to the organization and without introduction additional costs for the ED or the GPP. In addition, a reusable simulation tool is embedded within the hospital with staff trained to use this, allowing for continuous improvements of the IEP.

Finally, based on the results of this study, a pilot project of one of the interventions, letting physician assistants treat patients at both the GPP and ED, 
has been trialed in practice, showing an improvement that closely matches the prediction resulting from our simulation model. Future work will involve modeling the interaction between the IEP and other departments in the hospital, such that the entire care pathway chain can be optimized. 


\section{Appointment scheduling with unscheduled arrivals and reprioritization}

\subsection{Introduction}

We study the optimization of an appointment scheduling problem with unscheduled arrivals and reprioritization. This research was inspired by the HagaZiekenhuis, a Dutch hospital where such situations are encountered in the radiology department. Radiology departments offer diagnostic services to other hospital departments, and outside healthcare providers. In addition to outpatients who receive an appointment, diagnostics requests are also received from the emergency department and the wards. Of these requests, some patients require immediate attention, and should be diagnosed as soon as possible, while others are urgent but may wait for some time. These patients however must be seen within a given time frame. We shall refer to these patients as semi-urgent.

The problem we study can also be found in outpatient departments that are faced with patients with appointments, as well as unscheduled arrivals with varying degrees of urgency [94]. Our contribution is that we build upon work in appointment scheduling by incorporating this reprioritization effect, and apply our approach to both theoretical instances and a case study. To this end, we use Discrete Event Simulation (DES) as well as a constructive and local search heuristic to find appointment schedules that minimize waiting times. In addition, we compare the simulation model outcomes with results obtained from an exact model, and test our search heuristic, before applying it to a case study.

The structure of this chapter is as follows. Section 4.2 gives a problem description, and in Section 4.3 we review the literature. Section 4.4 describes our approach, and Section 4.5 details the simulation model and heuristics used to find and evaluate appointment schedules. In Section 4.6 we provide results of this research, using both theoretical instances and a case study from HagaZiekenhuis. Finally, Section 4.7 provides a discussion and conclusion. 


\section{Chapter 4. Appointment scheduling with unscheduled arrivals and reprioritization}

\subsection{Problem description}

In this chapter, we aim to evaluate and optimize an appointment schedule for a given day where part of the patients arrive via appointment, and others arrive without prior notice. Appointment scheduling is studied on both a tactical, as well as an operational level. On an operational level, models are used to find policies that specify which patients to prioritize in an upcoming time period (e.g., time slot or day), given the current state (e.g., number and urgency of waiting patients) and associated costs. In contrast, on a tactical level, the prioritization policies are assumed fixed, and the aim is to create appointment schedules for appointment patients such that, given a fixed prioritization policy, performance such as waiting times are minimized. Our problem falls within the second category, we assume the prioritization of patients is fixed, and we aim to create an appointment schedule that minimizes waiting times. While it may be beneficial to create situation dependent policies, in practice such policies may be difficult to implement, while it is simple to only schedule appointments given a new appointment schedule.

An appointment schedule consists of several time slots during the day where appointment patients are scheduled. Besides these known arrivals, there are time dependent unscheduled arrivals during the day. These unscheduled arrivals may vary in priority, some must be scheduled as soon as possible, while others have a set due time (e.g., must be diagnosed within 2 hours). However, if unscheduled patients are not seen, their due time comes closer and by this their urgency increases. When diagnostics become available, the next patient is selected based on his/her urgency. As such, patients are prioritized by their time remaining until due time, with the patient(s) with the least time until due time selected first (i.e., remaining slack time). Specifically, the prioritization is as follows:

1. Unscheduled patients at their due time, ordered by waiting time (longest waiting time first)

2. Scheduled patients that arrived via appointment

3. Unscheduled patients not at their due time, based on time remaining (closest first), and (in case of equal time until due time) on waiting time (longest waiting first).

\subsection{Literature}

Given the common use of appointment systems by healthcare providers it is not surprising that outpatient scheduling is a topic of interest and has been studied for a long time, starting with Bailey and Welch [248]. Those unfamiliar with the vast amount of earlier work on this topic we refer to Cayirli [41] who has provided an extensive literature review. As noted in Section 4.2, we consider outpatient scheduling on a tactical level, taking into account (time dependent) 
patient arrivals of multiple urgency types, and reprioritizations that take place when unscheduled patients are left waiting. Therefore, we look at recent work that incorporates multiple urgency types, as well as papers including both scheduled and unscheduled arrivals.

Recent work is done by Patrick and Puterman [197]. Herein both high priority inpatients, as well as lower priority outpatients must be scheduled for a CT scan. A policy is provided where capacity is reserved for each priority level, allowing for carrying over a portion of the unscheduled demand to the next day. In their case however, no reprioritization takes place. Patrick, et al. [198] model a diagnostic resource where patients of multiple priority classes may request diagnostics with the aim to allocate capacity (daily) among the different classes such that the number of patients exceeding their waiting time is minimized. In their case however, a scheduling policy is sought that governs how many patients (per class) to schedule per day. This differs from our problem in that the number of appointment patients is fixed, and we must distribute them throughout the day such that waiting times are minimized.

Similar to our problem, Kolisch and Sickinger [143] model a radiology department with both scheduled and unscheduled patients of multiple priorities. Modeling the problem as a Markov decision process, they dynamically allocate available capacity to the patients such that a given cost function is minimized, thus studying the problem on an operational level. A later paper by Sickinger and Kolisch [218] evaluates and searches for appointment schedules that minimize a cost function. Also in this work, both scheduled and unscheduled patients are taken into account, however the priority of patients herein is fixed.

Koeleman and Koole [141] evaluate and search for appointment schedules while considering emergency arrivals that have priority over appointment patients. These emergency arrivals are taken into account when constructing appointment schedules. Using a local search algorithm they find the optimal solution minimizing the weighted sum of overtime, idle time and waiting times. They expand upon this including late and early arrivals of patients [140], however in both papers there are only two patient classes, and there is no reprioritization of untreated patients.

Cayirli, et al. [42] evaluate different appointment schedules from literature using computer simulation. Herein they take into account no-shows, as well as walk-ins, which are given a lower priority than appointment patients. They extend upon this study with flexible appointment intervals (based on patient type) [43] and again evaluate several appointment schedules. In our case however, we aim to to find the best possible appointment schedule, and not evaluate several schedule possibilities. In addition, in both papers no reprioritization of patients is taken into account.

Kortbeek et al. [146] present an approach for optimizing appointment schedules for outpatient clinics with both scheduled and unscheduled arrivals. Herein they use two models to determine both the number of appointments to be offered over a planning horizon, and the times during the day these appointments 


\section{Chapter 4. Appointment scheduling with unscheduled arrivals and reprioritization}

should be offered. The latter is similar to our problem, but they model only one unscheduled patient type, and ignore the reprioritization of patients. Using a local search heuristic they iteratively improve the quality of the appointment schedule.

Our contribution is threefold. First we build on recent work in appointment scheduling in healthcare by incorporating different patient types (both scheduled and unscheduled), as well as urgency levels. Herein we consider the reprioritization of unscheduled patients, reflecting that if lower urgency unscheduled patients wait too long, they will be prioritized over appointment patients. To our knowledge, this reprioritization has so far not been addressed in a similar problem setting, while often seen in practice. Second, we use a generic simulation model and generic search heuristics to systematically evaluate appointment schedules and search for good appointment schedules. Both the model and the heuristics are easily adapted to include other scenario specific aspects (e.g., no shows) and can thus be applied to other (healthcare) settings. In addition we verify our approach with a Markov Reward Process, which may be applied itself to smaller instances. Finally, our approach enables healthcare providers to create more balanced appointment schedules in a timely manner, taking into account waiting time for both elective and urgent patients, while taking into account the reprioritizations that take place in healthcare settings.

\subsection{Assumptions and Approach}

In this section we detail the assumptions made in modeling the radiology department, as well as the approach taken to evaluate and optimize appointment schedules.

Our approach builds upon the approach by Kortbeek et al. [146], wherein we (1) need a method to evaluate the performance of an appointment schedule, and (2) a local search approach which incorporates the aforementioned method and iteratively optimizes the schedule. The underlying assumptions of this approach are as follows. We divide a day into $T$ time slots of equal length $h$, with $C$ servers (e.g., CT scanners) available every day. The division of the day into slots of fixed length is motivated by the fact that, in practice, radiology appointment lengths are reduced in variability, as preparatory steps, such as administering contrast fluid [60], are externalized, and better protocols and reconfiguration times are established.

We assume scheduled patients arrive on time for their appointment, and that all diagnostics (both scheduled and unscheduled) require one time slot. Unscheduled patients that arrive during the day may have different urgencies, reflected by a due time (i.e., time slot in which they ultimately must be seen). Let $R$ be the number of time slots a patient of the lowest urgency may wait, then unscheduled patients have a time until due time of $r$, with $r=0, \ldots, R$. We assume unscheduled patients arrive via a non-stationary Poisson arrival process denoted by $\lambda_{t r}$, for time slot $t=1, \ldots, T$, and (time until) due time $r$. 
Table 4.1 Notation introduced in Section 4.4

\begin{tabular}{ll}
\hline Symbol & Description \\
\hline$T$ & Number of time slots during a day \\
$t$ & Time slot index $(t=1, \ldots, T)$ \\
$h$ & Length of a time slot \\
$C$ & Number of CT scanners (resources) \\
$R$ & Highest patient due time (i.e., lowest urgency) \\
$r$ & Due time index $(r=0, \ldots, R)$ \\
$\lambda_{t r}$ & Arrival rate of unscheduled patient with due time $r$ during time slot \\
& {$[t-1, t]$} \\
$x_{t}$ & Number of appointments patients that arrive for slot $t$ \\
\hline
\end{tabular}

Unscheduled patients whose due time has not passed wait for scheduled patients, and all other patients that arrived earlier, with an earlier or equal due time, as well as patients with an earlier due time that may arrive during their wait. Once an unscheduled patient is at their due time, their priority no longer increases, and they are served as soon as possible. This means that they only wait for current patients being diagnosed, other patients that reached their due time earlier, and in case of equal due time, patients that arrived earlier. Note that urgent patients may also arrive that should be served as soon as possible (i.e., their due time starts at 0). Finally, scheduled patients wait for all unscheduled patients at or past their due time, as well as other scheduled patients that arrived earlier. During the day, appointments are also scheduled in the time slots, and the number of patients scheduled for slot $t$ is denoted by $x_{t}$. An appointment schedule for the day is then described by: $\mathbf{x}=\left(x_{1}, \ldots, x_{T}\right)$. The notation introduced in this section is listed in Table 4.1 .

We use Discrete Event Simulation (DES) to evaluate the performance of an appointment schedule, paired with constructive and Tabu local search heuristics to search for appointment schedules that minimize waiting times. We discuss the simulation model as well as the heuristics in Section 4.5. To verify our DES, we also model the radiology department as a Markov Reward Process (MRP), wherein a time slot corresponds to a single diagnostic session (both appointment and unscheduled) during which patients are diagnosed. This MRP is detailed and discussed in the appendix.

The use of simulation modeling is motivated by the fact that the state space quickly expands, and quickly becomes intractable using the MRP, making it impossible to evaluate a single appointment schedule for a realistic case instance, let alone search for the optimal schedule. When verifying our simulation model, we compare simulation outcomes of small test instances with the MRP model results. In addition, we use the MRP to find the optimal appointment schedules for the test instances. This is done by enumerating all possible schedules. We evaluate the effectiveness of our simulation model and heuristics by comparing results to those of the optimal schedules. Section 4.6.1 describes the used test 
instances, and details of the MRP are included in the Appendix.

\subsection{Simulation model and search heuristics}

In this section we describe the simulation model (4.5.1), as well as the performance criteria used when evaluating appointment schedules (4.5.2). Following this we present the constructive and local search heuristics for optimizing the appointment schedule (4.5.3).

\subsubsection{Simulation model}

Within the simulation model, a day is simulated by "jumping through" (the start of) the time slots $(t)$ that make up a day. At the start of every time slot, scheduled patients arrive based on the appointment schedule $x_{t}$, and according to the unscheduled patient arrival rates $\lambda_{t r}$. Similar to the opening and closing of a radiology department the model stops when all patients that have arrived in regular time have been treated, and there are no more patients left. Events take place in the following order:

1. Patient arrivals (scheduled and unscheduled) are determined

2. Patients are selected to be treated (following the prioritization detailed in Section 4.2)

3. Urgencies of patients not treated are updated

A patient list contains the queue of patients currently present in the system with all relevant information (e.g., arrival time, initial urgency, etc.). When a patient is treated, the waiting time is recorded. When new patients arrive, they are placed at their proper place within the patient list.

To determine the number of simulation runs, we perform sufficient simulations, such that the specified precision of the time slot with the highest variability (of waiting time) in a schedule has at most a relative error of $5 \%$, with a confidence level of $95 \%$ [135]. We initialize the number of simulation runs (i.e., days) to 20.000 , and use common random numbers when evaluating different schedules. With the initial number of simulation runs we find that all time slots with considerable waiting times fall within the specified precision. For the sparse slots where the waiting time is close to 0 this is not the case. Since we assess the performance of the schedule based on the maximum waiting time during the day (Section 4.7), these sparse slots have no impact on the objective. Therefore we do not increase the number of simulation runs. Note that while the simulation model outcomes are estimates, with the specified relative error of at most $5 \%$, all subsequent comparisons between heuristic outcomes and current situation in Section 4.6 are found to significantly differ and we omit the confidence intervals for brevity. In addition, we verify the simulation model results with those obtained 
by the MRP, and find that all MRP results are enclosed within the simulation model confidence intervals.

\subsubsection{Performance criteria}

To evaluate the performance of an appointment schedule we are interested in the waiting time of both scheduled and unscheduled patients. Specifically, we want to minimize, and distribute evenly, the waiting time for scheduled patients (caused by unscheduled arrivals). In addition, the appointment schedule should be such that most unscheduled patients are seen on time. Therefore, we also require that a percentage of unscheduled patients, specified by a pre-set norm, are seen before their stated due time. We denote this on time percentage as OTP. We formulate $\mathbb{E}\left[W^{t}\right]$ as the expected waiting time of a scheduled appointment patient arriving at time slot $t$. In addition, we denote $V_{t, r}$ as the probability that an unscheduled patient arriving at time $t$, with initial time until the due time of $r$ slots, is not treated on time. Our objective is then formulated as follows:

$$
\begin{aligned}
& \min \max _{t} \mathbb{E}\left[W^{t}\right] \\
& \text { s.t. } \\
& V_{t, r}<1-O T P \quad \forall t, r
\end{aligned}
$$

\subsubsection{Constructive and local search heuristics}

To optimize the appointment schedule, a constructive heuristic generates an initial appointment schedule, after which a local search heuristic improves (upon) it. Given the goal of minimizing the maximum expected waiting time during the day, the constructive heuristic starts with an empty appointment schedule $\mathbf{x}_{t}=0, t \in\{1, . ., T\}$, and iteratively adds one appointment to the time slot $t^{\prime}:=\arg \min \max \mathbb{E}\left[W^{t}\right]$ until all required appointment slots $K$ have been assigned. In other words, starting with an empty schedule, the effect of adding an appointment to a slot is evaluated for every possible time slot. Then the appointment is added to the time slot that results in the lowest maximum waiting time encountered during the day, and the next appointment is similarly added. As such, given a current schedule, the appointment is added to the best available slot.

As we aim to minimize the maximum expected waiting time, in the optimal schedule the waiting time (per time slot) is spread out evenly across the day. Therefore, it is likely that moving an appointment slot from a busy slot to a quiet slot improves the schedule's performance. We therefore perform a Tabu search as follows. We denote $v$ "from slots" and $w$ "to slots" $\left(v, w \in \mathbb{N}^{+}\right)$, which are, respectively, the time slots with the highest and lowest expected waiting time $\left(\mathbb{E}\left[W^{t}\right]\right)$. Moving an appointment from a high to low waiting time slot is then a neighbor solution, with the neighborhood consisting of all possible moves, 


\section{Chapter 4. Appointment scheduling with unscheduled arrivals and reprioritization}

specifically $v \cdot w$ solutions. Our Tabu search then accepts the best neighbor solution that is not tabu, and adds it to the tabu list of size $L^{\text {size }}$. This is repeated until $r$ iterations have been done, or no feasible solution is found. We experimented with several local search techniques, and Tabu search settings, and found this approach best performing with respect to computation time and outcomes.

\subsection{Experiments and results}

To evaluate the performance of our local search heuristic we first apply it to small test instances, and compare performance with the optimal solution obtained from the enumerated results of the MRP model. Using test instances with varying parameter settings not only allows us to compare simulation results with the MRP, but also evaluate the heuristics under more general settings. In addition, we apply our approach to a Haga case study where both appointment patients are scheduled, and urgent arrivals take place. This section first details the input parameters of the artificial test instances (4.6.1), followed by numerical results of the test instances (4.6.2). Following this, we present the case study (4.6.3), and numerical results of applying the heuristic approach to the case study (4.6.4). Results in this section are obtained using the simulation model from Section 4.5.1, and in case of the test instances, compared with the (optimal) results from the MRP. Programming was done using the Delphi programming language from CodeGear and all experiments were run on an Intel $2.4 \mathrm{GHz} \mathrm{PC}$ with $4 \mathrm{~GB}$ of RAM.

\subsubsection{Input parameters test instances}

In our test instances we consider a department with two resources $(C=2)$, and a day consisting of 8 time slots $(T=8)$. We vary the arrival patterns of unscheduled arrivals during the day, with two types of unscheduled arrivals. Specifically, $\lambda_{t, 0}$ is the arrival rate of urgent patients (at time $t$ ), and $\lambda_{t, R}$ the arrival rate of unscheduled patients that may wait for $R$ time periods (due time: $t+R$ ). In our test instances, each time slot has similar arrival rates for the two unscheduled arrival types $\left(\lambda_{t, 0}=\lambda_{t, R}, \forall t\right)$. The considered arrival patterns are shown in Figure 4.1.

Arrival pattern 1 resembles the arrival rates as seen in practice, albeit at a smaller time scale, where arrivals increase into the start of the afternoon, and then decrease back to just above the start-of-day arrival rates. In addition, we evaluate an arrival pattern (2) with two peaks, where walk-ins are more likely to arrive at the start or end of the day (e.g., walk-in blood donations before or after work). Another arrival pattern encountered in practice may be a high initial arrival rate which decreases during the day (pattern 5), resembling waiting patients that arrived overnight and are waiting to see a healthcare provider. Finally, we also evaluate an arrival pattern with ever increasing arrival rates (pattern 3) 
Figure 4.1 Unscheduled patient arrival rates per time slot

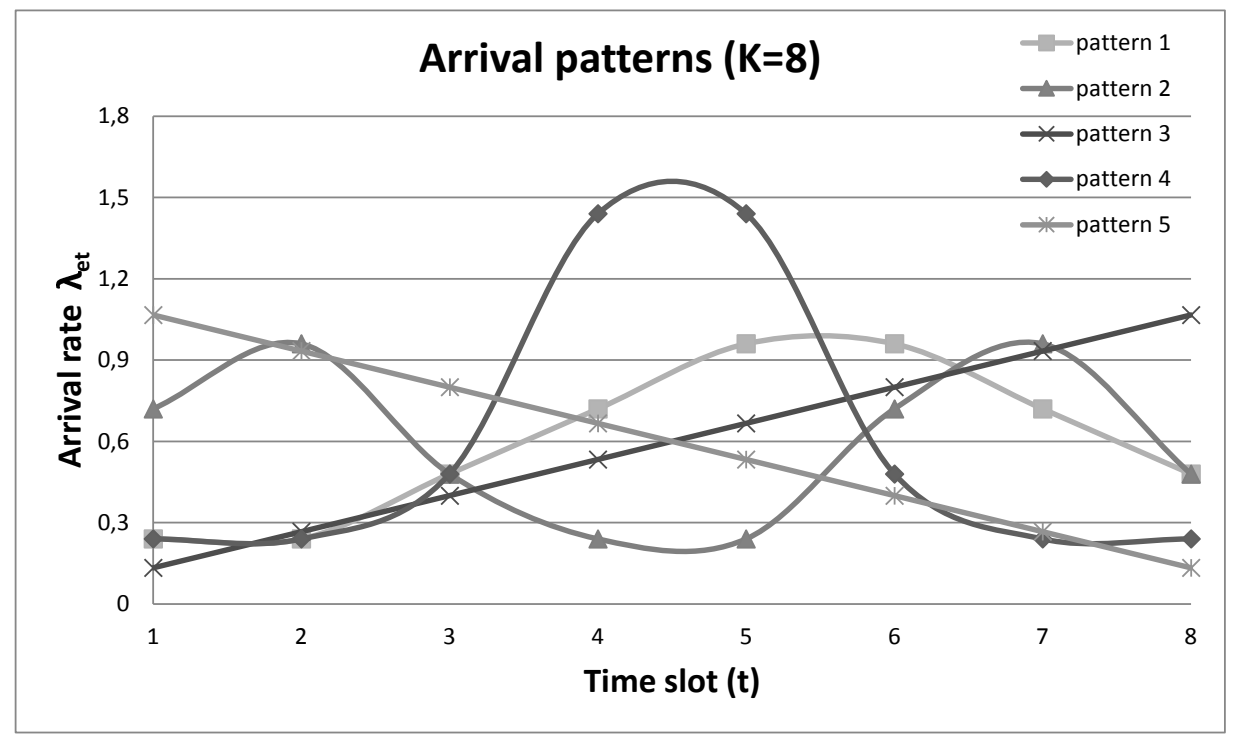

and a single (very) large peak (pattern 4) to evaluate heuristic performance under diverse arrival scenarios. We vary the urgency of the less urgent patient that may wait $R$ slots, with $R \in\{1,3\}$. In addition we vary the number of appointment patients $K$ that should be scheduled, with $K \in\{5,8\}$. When changing $K$, we correct our unscheduled arrivals accordingly, such that overall utilization is approximately $80 \%$. Finally, we set the on time probability OTP at 0.75 . Both fixed and varied inputs, as well as Tabu search settings are listed in Table 4.2. In total we evaluate 20 test instances.

Table 4.2 Fixed and varied input parameters

\begin{tabular}{lll}
\hline Parameters & Description & Value $(s)$ \\
\hline$T$ & time of day & 8 \\
$S$ & number of resources & 2 \\
OTP & \% of unscheduled patients that must be seen on time & 0.75 \\
$v$ & number of from slots & 3 \\
$w$ & number of to slots & 3 \\
$L^{\text {size }}$ & Tabu list size & 10 \\
$r$ & number of local search iterations & 200 \\
\hline \hline$K$ & number of appointments to schedule & $\{5,8\}$ \\
$R$ & highest due time (in slots) of unscheduled arrivals & $\{1,3\}$ \\
$\lambda_{t r}$ & arrival pattern of unscheduled patients & pattern: $\{1,2,3,4,5\}$ \\
\hline
\end{tabular}




\section{Chapter 4. Appointment scheduling with unscheduled arrivals and reprioritization}

\subsubsection{Results test instances}

In this section we discuss the results of the local search heuristic regarding the test instances. Table 4.3 contains the outcomes of the search heuristic per instance. Per test instance the maximum expected waiting time $\left(\mathbb{E}\left[W^{t}\right]\right)$ for appointment patients arriving during the day is given for the schedule found by the constructive and local search. To evaluate the effectiveness of our approach we compare the outcomes with the optimal schedules. Also, we compare the found schedules with the performance of the scheduling policy used in practice. Currently, appointments are booked every other time slot (e.g., 2,0,2,0,...)). Finally, we show the additional improvement made by the local search heuristic over the constructive heuristic, as well as the runtime of the search heuristics.

Most schedules plan patients when the unscheduled arrival rate is low, such that the overall arrival rate of patients is leveled. The exceptions to this are the instances with arrival pattern 2. We illustrate this in Figure 4.2, which concerns test instance 5 . Here we see the arrival pattern, the number of appointments and the expected waiting time for scheduled patients. While this pattern has a peak at the start of the day, still patients are planned during these time slots. This makes sense as it takes time to have enough unscheduled patients to push back appointments. Also, we observe that if the maximum initial due time is high $(\mathrm{R}=3)$, appointments are spread more over the day, as this allows unscheduled patients to wait longer and fill gaps in the schedule while still being treated on time.

Figure 4.2 Performance best found schedule for instance 5

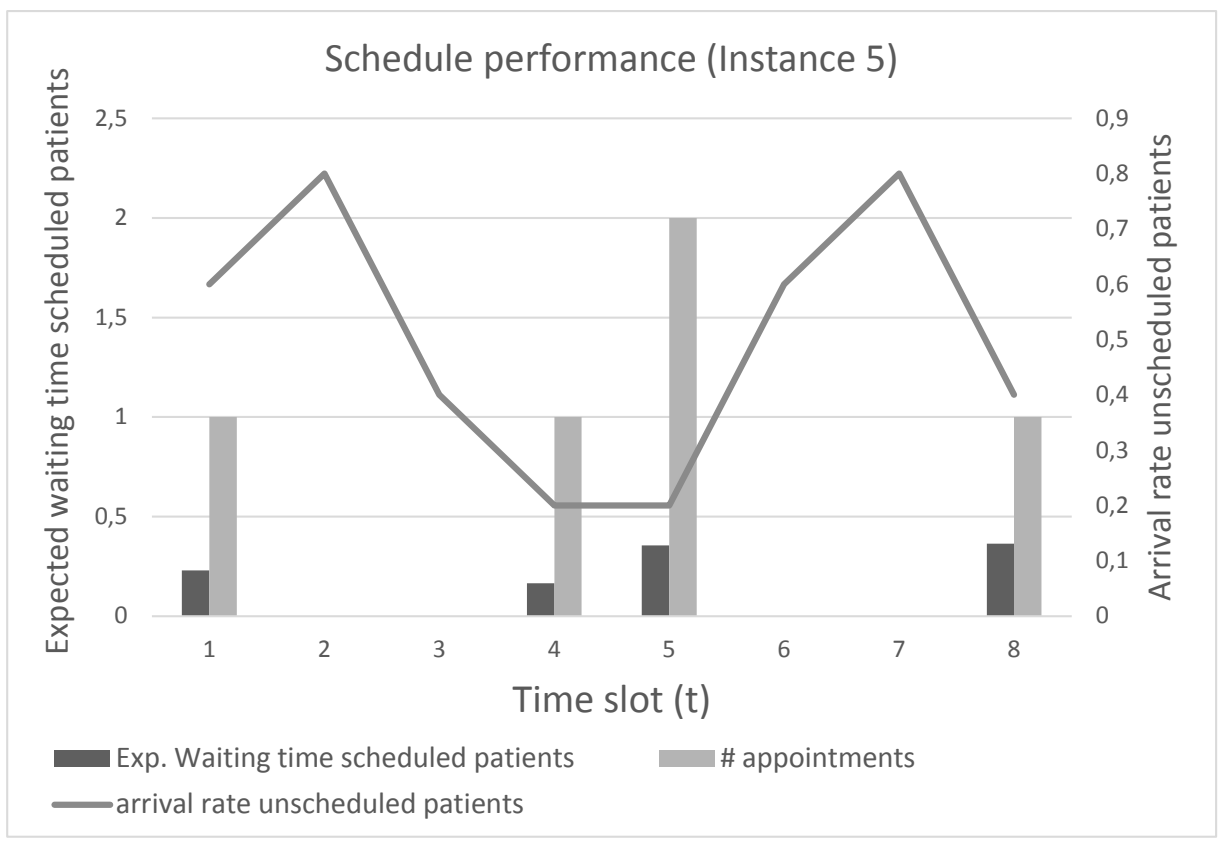


We note that instance 13 has no feasible schedules. As this instance has a large peak in the middle of the day of unscheduled arrivals, all of which are (very) urgent, no schedule can guarantee that $75 \%$ of unscheduled patients are seen on time. In all other cases, the constructive and local search heuristic found the optimal schedule, or a good schedule in the case of instance 8. Here the found schedule plans one patient every slot, while the optimal schedule plans two patients in time slot four, and none in slot five (i.e., $\{1,1,1,2,0,1,1,1\}$ ). In Table 4.3 we also list the improvement (i.e., reduction of max. waiting time) of the schedules that is achieved by the local search heuristic over the initial constructive heuristic solution. We thus observe that the constructive heuristic is very effective for these small instances, in 11 out of 19 instances the optimal schedule is found. In addition, we see that our approach achieves considerable improvement over the current scheduling policy used in practice of booking appointments every other slot (e.g., $(2,0,2,0, \ldots))$. Note that "inf" denotes instances where the base policy did not meet the on time probability constraint of unscheduled patients, and direct comparison is not possible. We conclude that the heuristic approach seems to find good schedules and apply it to our case study in the next section. 


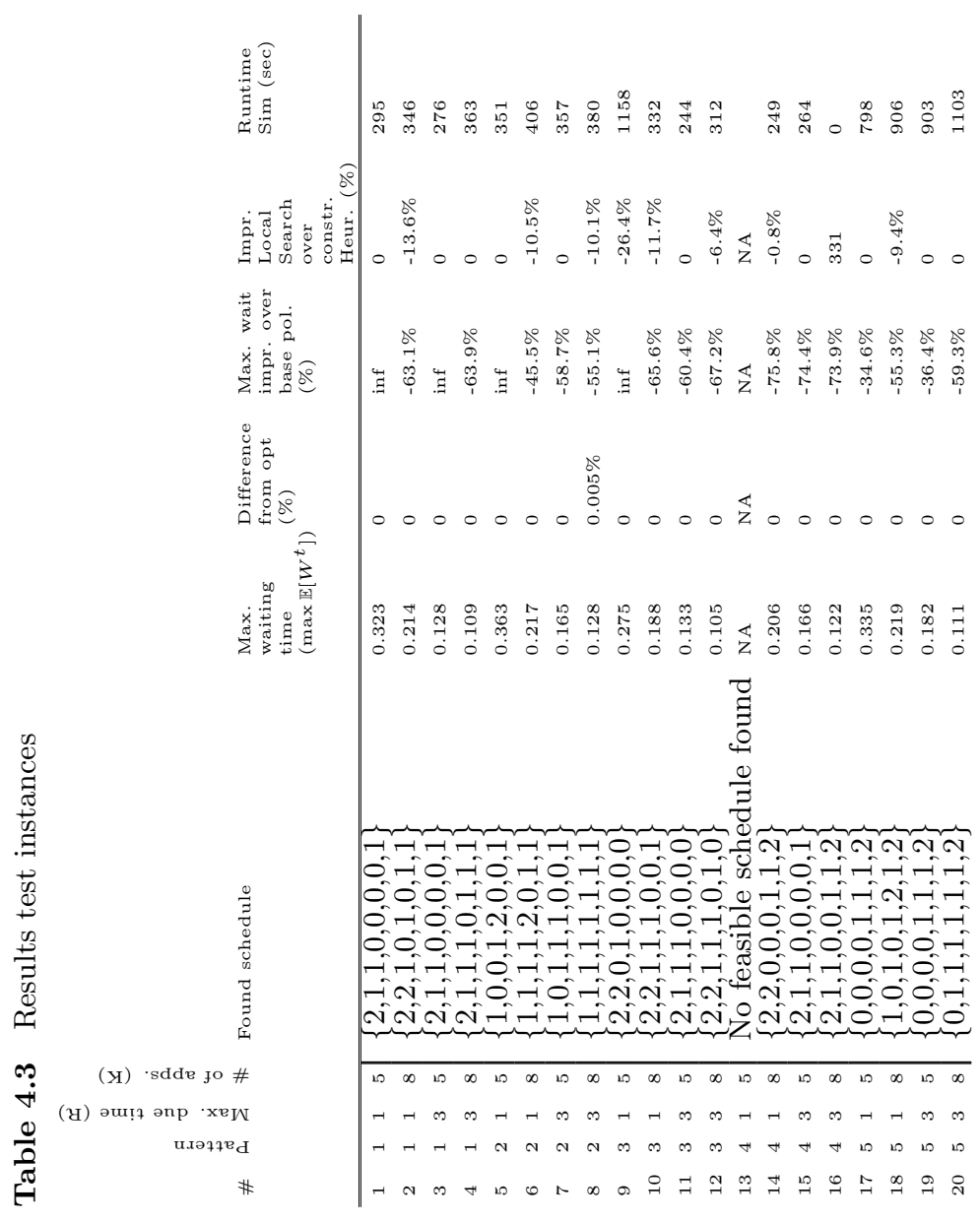




\subsubsection{Case study instance}

HagaZiekenhuis has three CT scanners $(C=3)$, for which appointments are offered in 15 minute slots. Regular opening hours of the department are from 8:00 to $16: 30$, resulting in 34 time slots per scanner $(T=34)$. Currently, every other time slot is reserved for scheduled patients. In practice, however, not all appointment slots are utilized, with the average number of appointments per day at $K=36$. In addition there are unscheduled arrivals consisting of patients that must be seen as soon as possible, and patients that may wait for some time ( 2 hours, $R=8$ ). To estimate the unscheduled arrival rates, data of the total number of arrivals at the radiology department has been combined with arrival information from the Emergency Department. Figure 4.3 shows the arrival rates of unscheduled patients during the day. As available information was hourly based, the arrival rates per slot equal the hourly rates divided by four. The arrival pattern clearly follows (with a delay) the typical arrival pattern of the ED. In total, the utilization of the system is $77.2 \%$. In addition, the required probability of unscheduled patients being treated on time is set at $90 \%(O T P=0.9)$.

Figure 4.3 Unscheduled patient arrival rates per time slot

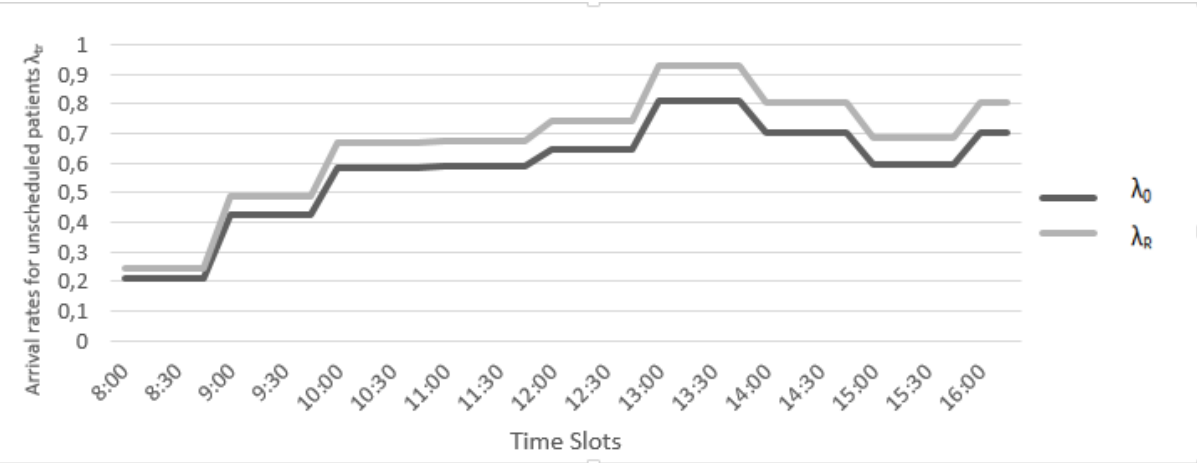

\subsubsection{Results case study}

This section presents the results of the case study. We first apply our approach to the current situation, and evaluate the found schedule. Also, we evaluate the effect of the appointment schedule on additional performance indicators. These are waiting time for unscheduled patients, utilization of CT scanners during the day, and overtime. Following the evaluation of the current situation, we then evaluate a what-if scenario with an increased utilization, where $20 \%$ more appointment patients are to be scheduled, to investigate performance with a potential increase of patient demand.

The runtime for the constructive and local search heuristic in the current scenario is 101.3 minutes, which is reasonable given that creating an appointment schedule only needs to be done every few months. Table 4.4 gives the performance 


\section{Chapter 4. Appointment scheduling with unscheduled arrivals and reprioritization}

of the current schedule, as well as the schedule found by the heuristic for the current situation. In the new schedule, more patients are scheduled at the start of the day. Following this, patients are scheduled evenly throughout the day, with a gap when there are many unscheduled arrivals expected. Similar to the test instances, appointments are spread out more over the available time slots, which reduces the peaks in scheduled patient waiting times. This makes sense, as for the time slots when three appointments are scheduled, only a single unscheduled (urgent) patient arriving causes one of the appointment patients to wait. The proposed schedule shows a reduction of the maximum (expected) waiting time during the day from 0.148 slots, to 0.046 slots ( $69 \%$ reduction), in comparison with the policy currently used in practice.

Table 4.4 Results case study (current situation)

\begin{tabular}{c|cc}
\hline Schedule & $\max \mathbb{E}\left[W^{t}\right]$ & schedule $(x)$ \\
\hline Current & 0.148 & $\{3,0,3,0,2,0,2,0,2,0,2,0,2,0,2,0,2,0,2,0,2,0,2,0,2,0,2,0,2,0,2,0,2,0\}$ \\
Heuristic & 0.046 & $\{2,2,2,2,1,2,1,2,1,1,1,1,1,1,1,1,1,1,1,1,0,0,0,0,1,1,1,1,1,1,1,1,1,1\}$ \\
\hline
\end{tabular}

Figure 4.4 shows the waiting times specified throughout the day for appointment patients, for both the current and found schedule. In addition the arrival rates per time slot are displayed. Besides the reduction of the maximum expected waiting time, the even distribution also ensures a fairer distribution of waiting time across appointment slots. Under the current schedule, it is most beneficial to have an appointment just after the start of the day (around 9AM), as the expected waiting time is considerably higher for appointments during the busiest moments in the afternoon. Conversely, this effect is lessened under the new schedule.

When evaluating the waiting times for urgent patients that should be seen as soon as possible we found that the differences between schedules were less prevalent, as these patients are always immediately prioritized over the lower urgency and appointment patients. We find that, for the current schedule, the fraction of unscheduled patients that are seen before their stated due time falls within the stated OTP norm (90\%), similar to the found schedule result.

We also evaluate the effect of the new schedule on waiting time for lower urgency unscheduled patients. Figure 4.5 shows the expected waiting time for the lower urgency patients, that should be seen within two hours (i.e., 8 slots). We see that during the start of the day the expected waiting time is higher than under the current schedule. However, this stabilizes towards the afternoon. In contrast, later during the day the waiting time under the new schedule is at its lowest when no appointments are offered, and then remains below the waiting time of all time slots under the current schedule. From this we find that the new appointment schedule is not only beneficial for scheduled patients, it also reduces the average waiting time for unscheduled patients.

Besides the change in waiting times for appointment patients, the found appointment schedule also has an effect on utilization and overtime. As patients are 
Figure 4.4 Appointment patients expected waiting time per time slot $(\mathrm{K}=36)$

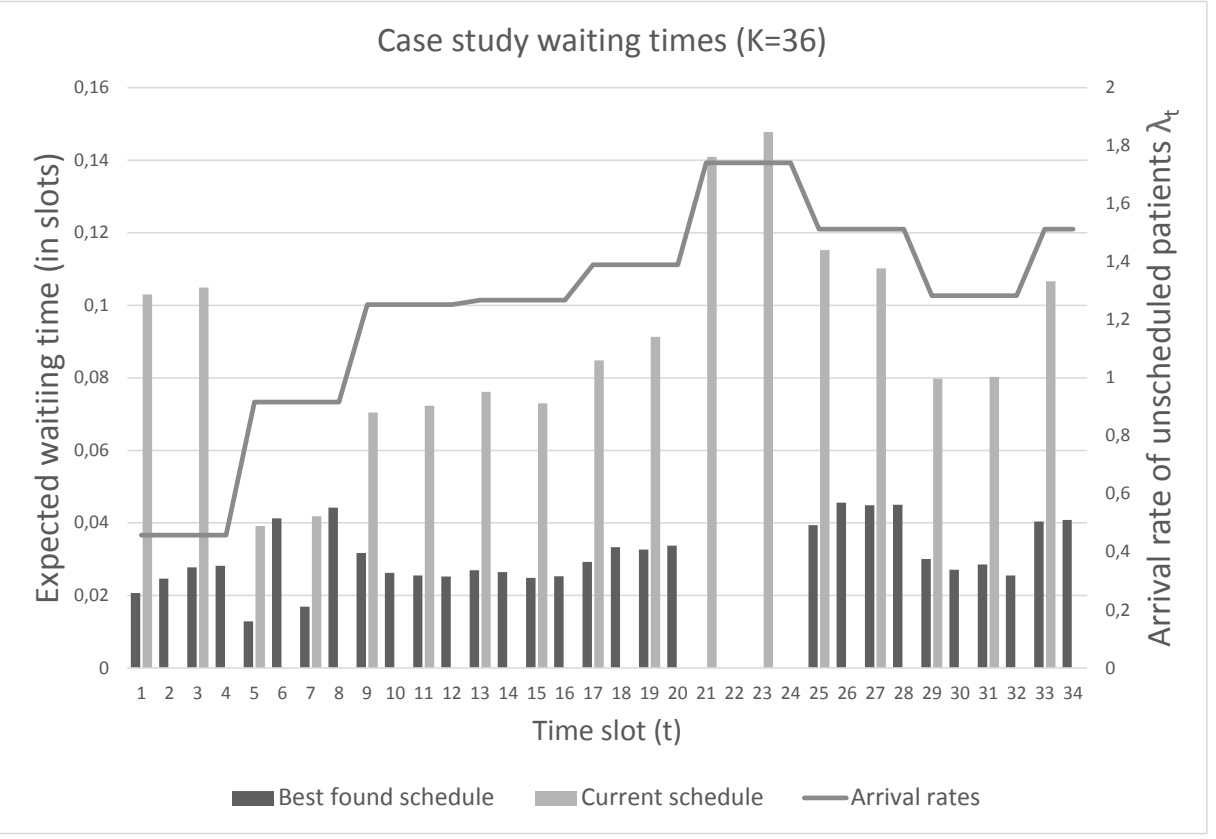

scheduled more evenly across the day, not only are waiting times reduced, but also the utilization and workload during the day is more evenly spread. Comparing the schedules, we see that the under the current schedule the system is fully utilized when scheduled patients arrive, and during the slots with no scheduled arrivals the utilization steadily increases into the afternoon until it stabilizes. The new schedule however almost immediately stabilizes, and shows a drop in utilization when more unscheduled patients are expected, ensuring that unscheduled patients are seen on time.

Finally, Figure 4.6 shows the overtime occurrence of both schedules. Specifically, it shows the fraction of times that the last patient is treated in the last time slot during regular time $(t=T)$, and later time slots. We see that under the current schedule, $70 \%$ of the time the last patient is treated during regular time, and there is no overtime. With the new schedule this reduces to $65 \%$. When patients are treated in overtime, this mostly extends to a single slot, which corresponds to an appointment of 15 minutes. Under both schedules, in less than $5 \%$ of the days the overtime is two slots or more. As the new schedule improves waiting times for both scheduled and unscheduled patients, this does come at a cost with regards to overtime.

In addition to the current situation we also ran the heuristic for a scenario where eight more appointments should be scheduled during the day, resulting in a utilization of $85.5 \%$. The runtime for this scenario was 115.7 minutes. Table 4.5 shows the outcome of the scenario using the current schedule and the heuristic. 
Chapter 4. Appointment scheduling with unscheduled arrivals and reprioritization

Figure 4.5 Average waiting time of unscheduled patients that must be seen within two hours $(\mathrm{r}=8, \mathrm{~K}=36)$

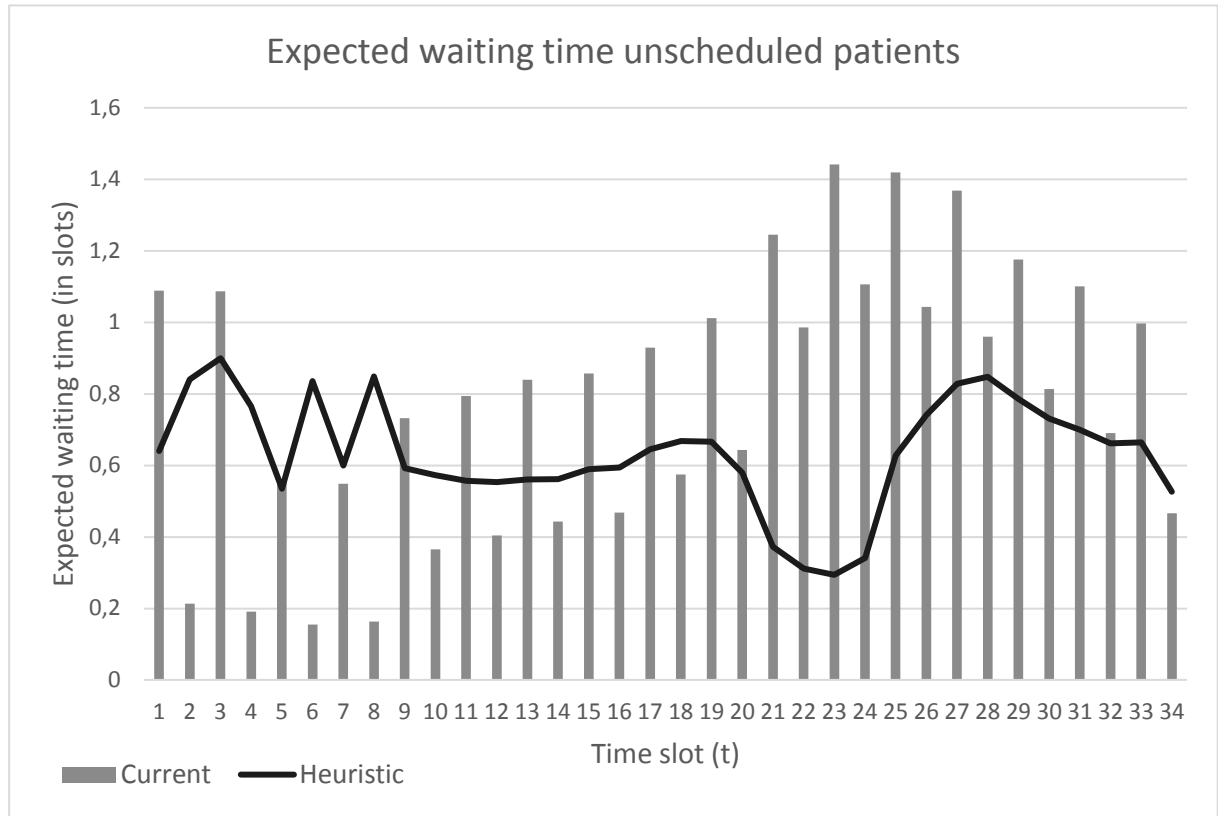

Figure 4.6 Probabilities that the last patient treated falls outside regular hours $(\mathrm{K}=36)$

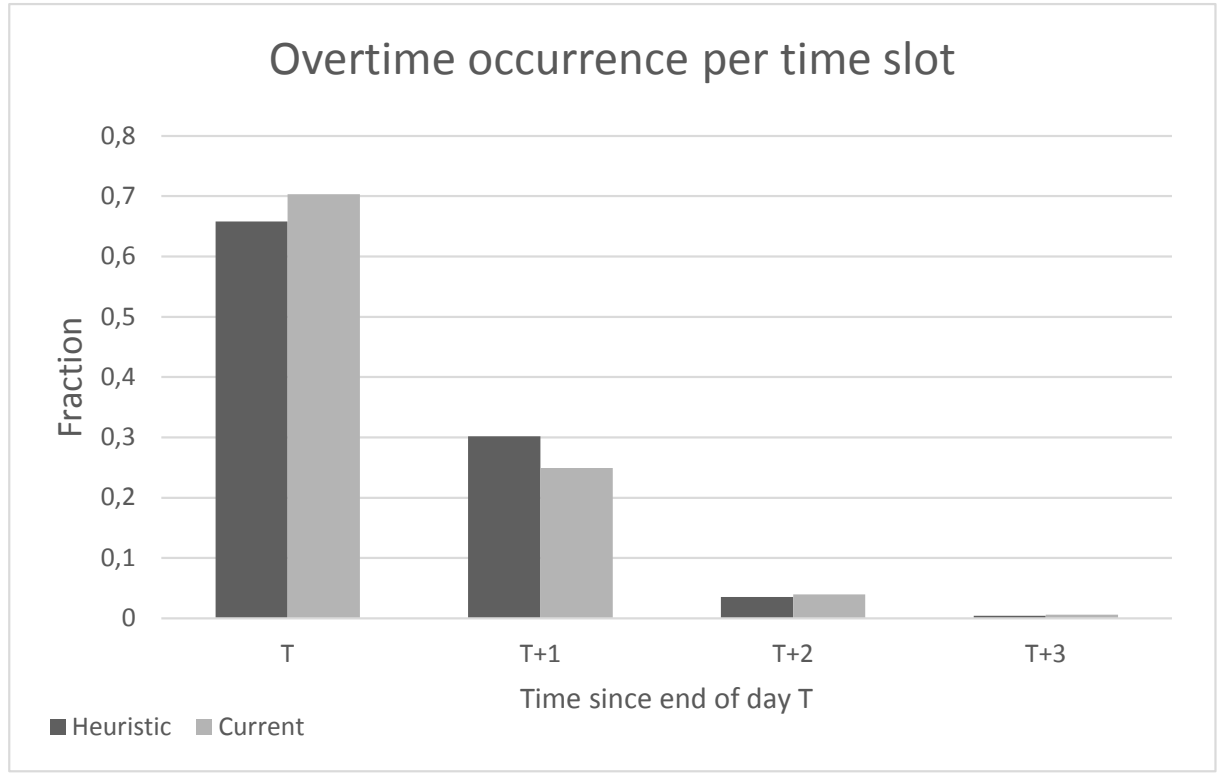


Similar to the current situation more patients are scheduled at the start of the day, and the remaining patients are scheduled evenly across the day. The maximum expected waiting time during the day is currently 0.148 slots, and 0.081 under the increased load and adapted schedule. Thus, using our approach, future increases in patient demand may be handled with the current capacity while still having acceptable waiting times. We conclude that our approach finds a good schedule within an acceptable amount of time for realistic instances, that takes into account the time dependent arrivals and reprioritization that take place in practice.

Table 4.5 Results case study (Increased appointments)

\begin{tabular}{c|cc}
\hline Schedule & $\max \mathbb{E}\left[W^{t}\right]$ & schedule $(x)$ \\
\hline Current & 0.262 & $\{3,0,3,0,3,0,3,0,3,0,3,0,3,0,3,0,3,0,3,0,2,0,2,0,2,0,2,0,2,0,2,0,2,0\}$ \\
Heuristic & 0.081 & $\{2,2,2,2,2,2,2,2,1,1,2,1,1,1,1,2,1,1,1,1,1,1,1,1,1,1,1,1,1,1,1,1,1,1\}$ \\
\hline
\end{tabular}

\subsection{Discussion}

In this chapter we studied the optimization of an appointment schedule with time dependent unscheduled arrivals and this research was inspired by the HagaZiekenhuis hospital where such situations are encountered in the radiology department. To find appointment schedules that minimize patient waiting times in a timely manner, we use a Discrete Event Simulation model in combination with constructive and local search heuristics. To verify this model, and evaluate the performance of the heuristics, we also evaluate multiple test instances using a Markov Reward Process model and enumerate all possible appointment schedules to find the optimal solution. From this comparison we find that our simulation and heuristics approach is able to find the optimal appointment schedule in most test scenarios, and finds good schedules in the remaining scenarios. We also apply our approach to a case study and find that significant waiting time reductions may be possible by adapting the slots available for appointments to the arrival rates of unscheduled patients.

Besides the reduction in waiting time for scheduled patients, the new schedule also shows a reduction in waiting time for lower urgency unscheduled patients, while still treating high urgency patients in time. Also, the utilization of CT scanners is spread evenly during the day, resulting in a more leveled workload. In addition to the even inflow of patients into the radiology department, the new schedule also allows for a more stable outflow of unscheduled patients back to wards and the Emergency Department. By spreading appointments during the day, also a more equitable distribution of the waiting time is obtained, so that patients having an appointment in the afternoon do not wait much longer than those arriving in the morning.

When looking at the found appointment schedules for both the current and busier scenario, we see that at no point three appointments are scheduled, as then 


\section{Chapter 4. Appointment scheduling with unscheduled arrivals and reprioritization}

only a single high urgency arrival means that an appointment patient is pushed back and must wait. We also observe in all experiments that the constructive heuristic by itself is already very effective in finding good solutions, as it iteratively adds appointment patients to the next best available time slot. In practice, our model may be used to periodically evaluate the offered appointment schedule, and re-optimize if either scheduled or unscheduled patient arrivals change.

For future work it may be interesting to evaluate the stochasticity of service times in practice. While in our case study, using the fixed service time assumption allows us to also enumerate exact results in order to compare our heuristics, incorporating these stochastic service times in follow up research can be interesting to see if a similar approach works. In addition, there can be seasonality effects in the arrival rates of unscheduled patients, or day-to-day effects regarding arrivals from the ED. In this research we use a single (averaged) day of arrival rates from the ED as little data on arrival numbers was available. However, our model and approach may be run for individual days with their unique arrival patterns, in order to construct appointment schedules for specific days. Regarding the unscheduled patient arrivals from the wards, while these are currently assumed as given, it may also be interesting to investigate the effect on the radiology department of changing the patient rounds on the wards, and thus the arrival rates of patients from the wards. Another example of future research may be the inclusion of no-shows of patients. By including this into the model the appointment schedule may also anticipate for this effect. Furthermore, different patient types or priority rules could also be incorporated, investigating the effect of differently prioritizing patients on the appointment schedule.

Concluding, our model generates appointment schedules, taking into account both elective and unexpected arrivals of patients, and the possible reprioritization that takes place between different patient types, during which patients may overtake each other and offers a practical and usable solution for practitioners. Applying our approach to a case study, the found appointment schedule considerably reduces expected waiting time for scheduled patients, while still ensuring unscheduled patients are seen on time. Additionally, the appointment schedule more fairly spreads the waiting time over patients, and results in a more even utilization, and thus workload, across the day. 


\section{Appendix}

In this appendix we describe the Markov Reward Process (MRP) used to explicitly evaluate the day process and its performance. By enumerating all possible appointment schedules and evaluating them with the MRP, the optimal schedule may be found for the test instances described in Section 4.6.1. Using these optimal schedules we compare the effectiveness of the simulation model and heuristics approach in Section 4.6.2. As noted, the radiology department is modeled as a Markov Reward Process. In the MRP a time slot corresponds to a single diagnostic session (both appointment and unscheduled) during which patients are diagnosed. To formulate our model we first give the state formulation followed by transition probabilities.

\section{Model}

State formulation: To formulate our model, we use the notation introduced in Table 4.1, introduced in Section 4.4. In addition, we denote $u_{r}$ as the number of unscheduled patients with $r$ remaining time slots until due time. Also, $R$ denotes the highest time until due time an arriving unscheduled patient can have. Following this, $\mathbf{u}=\left(u_{0}, \ldots, u_{R}\right)$ denotes all waiting unscheduled patients with different possible due times. The state of the system is then denoted by the tuple $(t, a, \mathbf{u})$, with $a$ the number of scheduled (appointment) patients, and $\mathbf{u}$, the vector of unscheduled patients with respectively 0 to $R$ time slots remaining before their due time, at the beginning of time slot $t$. Specifically, it is the number of patients in the system after patient arrivals at time $t$, and prior to patient selection for treatment in time $t$. For example, $(3,1,(1,0,2))$, indicates that, at the start of time slot 3 , there are four patients in the system. These are, respectively, one scheduled patient, one unscheduled patient at (or past) their due time, and two patients that may wait two more time slots. Notation introduced in this appendix is given in Table 4.6.

Transition probabilities: Let the probability of going from state $(t, a, \mathbf{u})$ to $(t+1, b, \mathbf{f})$ be denoted by $P\left[(b, \mathbf{f})_{t+1} \mid(a, \mathbf{u})_{t}\right]$, and $p_{t}^{i}(n)$ the probability that for time slot $t, n$ patients arrive of type $i$, with $i=\left(a, u_{0}, . ., u_{R}\right)$. Note that for scheduled patients $p_{t}^{a}\left(x_{t}\right)=1$ (i.e., scheduled arrivals follow the appointment schedule). With an empty system, this gives:

$$
P\left[(b, \mathbf{f})_{t+1} \mid(a, \mathbf{u})_{t}\right]=p_{t+1}^{a}(b) \cdot p_{t+1}^{u_{0}}\left(f_{0}\right) \cdot p_{t+1}^{u_{1}}\left(f_{1}\right) \cdot \ldots \cdot p_{t+1}^{u_{R}}\left(f_{R}\right)
$$

To formulate the transition probabilities for a non-empty system we introduce $n^{a}$, and $n^{u_{r}}$ (for $r=0, \ldots, R$ ), which denote the number of treated scheduled and unscheduled patients with time until due time remaining of $r$ respectively. Using this, we are able to define the state of the system after starting treatment of patients, but before new arrivals come in. Given the state of the system and 


\section{Chapter 4. Appointment scheduling with unscheduled arrivals and reprioritization}

prioritization, the number of treated patients per type is determined as follows:

$$
\begin{aligned}
n^{u_{0}} & =\min \left\{C ; u_{0}\right\} & \\
n^{a} & =\min \left\{\max \left\{C-u_{0} ; 0\right\} ; a\right\} & \\
n^{u_{r}} & =\min \left\{C-n^{a}-\sum_{i=0}^{r-1} n_{i} ; n_{r}\right\}, & \text { for } \mathrm{r}=1, \ldots, R
\end{aligned}
$$

Continuing the previous example, suppose the state of the system is $(3,1,1,0,2)$ and there are two CT machines $(C=2)$, then the unscheduled patient at their due time, as well as the appointment patient are treated (e.g., $n^{u_{0}}=n^{a}=1$ ). Following this, the state of the system after starting treatment of patients, but before new arrivals, is $(4,0,0,2,0)$. Note that the two unscheduled patients are one time slot closer to their due time. Using the number of treated patients and state of the system at time $t$, the partial transition probabilities per patient type are as follows:

(scheduled patients)

$$
P\left[(b)_{t+1} \mid(a)_{t}\right]=\quad p_{t+1}^{a}\left(b+n_{a}-a\right)
$$

(unscheduled patients)

$$
\begin{array}{ll}
P\left[\left(f_{r}\right)_{t+1} \mid(\mathbf{u})_{t}\right]= & p_{t+1}^{u_{r}}\left(f_{r}-\left(u_{r+1}-n^{u_{r+1}}+u_{r}-n^{u_{r}}\right)\right), \text { for } r=0 \\
P\left[\left(f_{r}\right)_{t+1} \mid(\mathbf{u})_{t}\right]= & p_{t+1}^{u_{r}}\left(f_{r}-\left(u_{r+1}-n^{u_{r+1}}\right), \text { for } r=1, \ldots, R-1\right. \\
P\left[\left(f_{r}\right)_{t+1} \mid(\mathbf{u})_{t}\right]= & p_{t+1}^{u_{r}}\left(f_{r}\right), \text { for } r=R
\end{array}
$$

The transition probability $P\left[(b, f)_{t+1} \mid(a, u)_{t}\right]$ can then be constructed by multiplying the partial transition probabilities:

$$
\begin{aligned}
& P\left[(b, f)_{t+1} \mid(a, u)_{t}\right]=P\left[(b)_{t+1} \mid(a)_{t}\right] \cdot P\left[\left(f_{0}\right)_{t+1} \mid\left(u_{0}\right)_{t}\right] \cdot \ldots \\
& \quad \cdot P\left[\left(f_{r}\right)_{t+1} \mid\left(u_{r}\right)_{t}\right]
\end{aligned}
$$

\section{System performance}

To evaluate the performance of an appointment schedule we are interested in the waiting time of both scheduled and unscheduled patients. Specifically, we want to minimize, and distribute evenly, the waiting time for scheduled patients (caused by unscheduled arrivals), while a percentage, specified by a pre-set norm, of unscheduled patients are seen before their stated due time.

The waiting time of a patient depends on the state of the system at their time of arrival (i.e., the number of patients already present and of higher priority), as well as the number of patients that arrive later, but are still of higher priority. In the remainder of this subsection we first determine the arrival probabilities (i.e., 
Table 4.6 Notation introduced in Section 4.7

\begin{tabular}{ll}
\hline symbol & description \\
\hline$a$ & Number of appointment patients present in the system at \\
& the beginning of a time slot. \\
& Number of unscheduled patients with remaining due time of \\
$u_{r}$ & $r$ in the system at the beginning of a time slot \\
& Number of unscheduled patients in the system at the begin- \\
$\mathbf{u}$ & ning of a time slot, $\mathbf{u}=\left(u_{0}, \ldots, u_{R}\right)$ \\
& State of the system: at the start of time slot t, there are $a$ \\
& appointment and $\mathbf{u}$ unscheduled patients present \\
& $P\left[\right.$ number of arrivals of type $i,\left(i=a, u_{0}, . ., u_{R}\right)$, during slot \\
$p_{t}^{i}(n)$ & $[t-1, t]$ is $n]$ \\
$P\left[(b, \mathbf{f})_{t+1} \mid(a, \mathbf{u})_{t}\right]$ & $P[$ Transition probability from state $(t, a, \mathbf{u})$ to state $(t+$ \\
& $1, b, \mathbf{f})]$ \\
& Number of treated scheduled and unscheduled patients re- \\
$n^{a} ; n^{u_{r}}$ & spectively \\
&
\end{tabular}

the probability that an arriving patient sees a certain system state), and then the waiting time distributions conditioned on these encountered system states. Combining these two gives the complete waiting time distribution of a patient arriving at a certain time slot. The notation introduced in Section 4.7 is listed in Table 4.7 .

Arrival probabilities: To determine the waiting time for patients we need the state probabilities seen by patients as they arrive. Suppose that just after starting patient diagnostics there are $k$ patients of type $i\left(i=a, u_{0}, . . u_{R}\right)$. Then an arriving patient of type $i$ only sees $k$ patients if he is the only arrival, or is the first of multiple arrivals. The probability that an arriving patient sees a certain state can be calculated by conditioning on the number of arrivals during the slot.

We denote $O_{t}(a, \mathbf{u})$ as the probability that at the start of time slot $t$, there are $(a, \mathbf{u})$ patients present. Additionally, we denote $O_{t}^{\prime}(a, \mathbf{u})$ as the probability that at the start of slot $t$, after starting diagnostics and before new arrivals, there are (still) $(a, \mathbf{u})$ patients present. Using $O_{t}(a, \mathbf{u})$ and $O_{t}^{\prime}(a, \mathbf{u})$ we can determine the arrival probability $Q_{t}^{i}(a, \mathbf{u})$, defined as the probability that a patient of type $i$, arriving for slot $t$, finds state $(a, \mathbf{u}) . O_{t}(a, \mathbf{u})$ and $O_{t}^{\prime}(a, \mathbf{u})$ are calculated as follows:

$$
\begin{aligned}
O_{1}(b, \mathbf{f}) & =p_{1}^{a}(b) \cdot p_{1}^{u_{0}}\left(f_{0}\right) \cdot p_{1}^{u_{1}}\left(f_{1}\right) \cdot \ldots \cdot p_{1}^{u_{R}}\left(f_{R}\right) \\
O_{t+1}(b, \mathbf{f}) & =\sum_{a} \sum_{u_{0}} \ldots \sum_{u_{R}}\left(O_{t}(a, u) \cdot P\left[(b, \mathbf{f})_{t+1} \mid(a, \mathbf{u})_{t}\right]\right),
\end{aligned}
$$

for $t=2, \ldots, T$ 


\section{Chapter 4. Appointment scheduling with unscheduled arrivals and reprioritization}

Let $n^{u}$ be the number of treated unscheduled patients, $n^{u}=\left(n^{u_{0}}, \ldots, n^{u_{R}}\right)$, then

$$
\begin{array}{r}
O_{t+1}^{\prime}(b, \mathbf{f})=\sum_{(a, \mathbf{u}) \mid\left[(a, \mathbf{u})-\left(n^{a}, n^{u}\right)=(b, \mathbf{f})\right]} O_{t+1}(a, \mathbf{u}), \\
\text { for } t=2, \ldots, T
\end{array}
$$

The probability that an arriving patient sees a certain state can be calculated by conditioning on the number of arrivals during the slot. Suppose the patient type under consideration are unscheduled patients with due time $0\left(u_{0}\right)$ patients, then:

$$
\begin{aligned}
& Q_{1}^{e_{0}}(b, \mathbf{f})=p_{1}^{a}(b) \cdot \frac{1}{\lambda_{1, u}} \sum_{n=f_{0}+1}^{\infty}\left[p_{1}^{u_{0}}(n)\right] \cdot p_{1}^{u_{1}}\left(f_{1}\right) \cdot \ldots \\
& \cdot p_{1}^{u_{R}}\left(f_{R}\right) \\
& Q_{t+1}^{u_{0}}(b, \mathbf{f})=\sum_{(a, \mathbf{u})}\left[O_{t}^{\prime}(a, \mathbf{u}) \cdot p_{t+1}^{a}(b-a)\right] \cdot \frac{1}{\lambda_{t+1, u}} \\
& \quad \sum_{n=f_{0}+1}^{\infty}\left[p_{t+1}^{u_{0}}(n)\right] \cdot p_{t+1}^{u_{1}}\left(f_{1}-u_{1}\right) \cdot \ldots \\
& \cdot p_{t+1}^{u_{R}}\left(f_{R}-u_{R}\right), \text { for } t=2, \ldots, T
\end{aligned}
$$

Note that, $\frac{1}{\lambda_{1, u}}$ denotes the average size of an arriving 'group' of patients during a time slot. Similarly these probabilities may be constructed for the other patient types. Using this we can determine the waiting time distributions for scheduled and unscheduled patients.

Conditional waiting time of scheduled patients: As mentioned, the waiting time of an appointment patient depends on the number of appointment patients already waiting in the system, the patients with a higher urgency in the system, as well as patients that may (arrive and) become higher urgency during their waiting time. We derive the conditional waiting time distribution of a patient, by conditioning on the state of the system when the appointment patient enters [167]. By setting the arrival rates of scheduled patients to $0\left(p^{a}(0)=1\right)$ for all future time periods, and defining all states where $\left(u_{0}+a\right)<C$ as absorbing states. Then, the time it takes to get to an absorbing state, is the waiting time of the scheduled patient in the original system.

For notational simplicity, we denote $\mathbf{c}=(b, \mathbf{f})$, as the state encountered by an arriving scheduled patient, and the state conditioned on. Following this we can calculate the time until absorption, as once an absorbing state is reached, the tagged (under evaluation) patient is treated. We denote $O_{\tau}^{t, \mathbf{c}, a}(a, \mathbf{u})$ as the probability that $\tau$ time slots after the tagged appointment patient's arrival in the conditioned state $\mathbf{c}$ and time $t$, the system is in state $(a, \mathbf{u})$. We then calculate 
$O_{\tau}^{t, \mathbf{c}, a}(a, \mathbf{u})$ as follows:

$$
\begin{aligned}
O_{0}^{t, \mathbf{c}, a}(\mathbf{c}) & =1 \\
O_{\tau+1}^{t, \mathbf{c}, a}(b, \mathbf{f}) & =\sum_{(a, \mathbf{u}) \mid\left(u_{0}+a\right) \geq C} O_{\tau}^{t, \mathbf{c}, a}(a, \mathbf{u}) . \\
P\left[(b, \mathbf{f})_{\tau+1} \mid(a, \mathbf{u})_{\tau}\right], \text { for } \tau \geq 1 &
\end{aligned}
$$

Note that $\left.P\left[(b, \mathbf{f})_{\tau+1} \mid(a, \mathbf{u})_{\tau}\right]\right)$ are the updated transition probabilities, where appointment patient arrivals are set to 0 , starting from the initial time of arrival $t$. Using this approach we can determine the waiting time distribution for a given arrival state. Let $W_{\tau}^{t}(b, \mathbf{f})$ be the probability that a scheduled patient that arrives in state $\mathbf{c}(=(b, \mathbf{f}))$ at time $t$ waits for $\tau$ time periods. This can be determined by:

$$
W_{\tau}^{t}(b, \mathbf{f})=\sum_{(a, \mathbf{u}) \mid\left(u_{0}+a\right)<C} O_{\tau}^{t, \mathbf{c}, a}(a, \mathbf{u}), \text { where } \mathbf{c}=(b, \mathbf{f})
$$

Combining (1) with the arrival probabilities allows for the formulation of $W_{\tau}^{t}$, the probability that an arriving appointment patient at time $t$, regardless of arrival state, waits for $\tau$ time periods. Also, we formulate $\mathbb{E}\left[W^{t}\right]$, the expected waiting time for an appointment patient arriving at time $t$ :

$$
\begin{aligned}
W_{\tau}^{t} & =\sum_{(b, \mathbf{f})} Q_{t}^{a}(b, \mathbf{f}) \cdot W_{\tau}^{t}(b, \mathbf{f}) \\
\mathbb{E}\left[W^{t}\right] & =\sum_{\tau=0}^{\infty} \tau \cdot W_{\tau}^{t}
\end{aligned}
$$

Lateness of unscheduled patients: Similar to calculating the waiting time of scheduled patients, we can determine the waiting time for unscheduled patients, and subsequently the lateness (waiting time past due time). Again we consider a system where the arrival rate of the unscheduled patient's type, as well as all lower urgency patient types, is set to 0 . In addition, as every unscheduled patient type that is not treated will be a higher urgency in the next time slot, we then also set the arrival rate of the tagged patient's new type to 0 , up to $u_{0}$.

Finally, as the arriving patient is prioritized over all lower urgency patients, from the patient's perspective these may be ignored (i.e., set to 0). Suppose the unscheduled patient's due time is $m$ slots from now, and arrives in state $(a, \mathbf{u})$, then the conditioned on state can be formulated by $c=(b, \mathbf{f})$, with $\left\{\begin{array}{l}b=a, \text { if } m \geq 0 \\ b=0, \text { otherwise }\end{array} \quad\right.$ and $\left\{\begin{array}{l}f_{r}=u_{r}, \text { if } r<m \\ f_{r}=u_{r}-1, \text { if } r=m \\ f_{r}=0, \text { if } r>m\end{array}\right.$

We denote $O_{\tau}^{t, \mathbf{c}, m}(a, \mathbf{u})$ as the probability that $\tau$ time slots after the tagged unscheduled patient's arrival in the conditioned state $\mathbf{c}$, at time $t$ and $m$ slots until 


\section{Chapter 4. Appointment scheduling with unscheduled arrivals and reprioritization}

due time, the system is in state $(a, \mathbf{u})$. We then calculate $O_{\tau}^{t, \mathbf{c}, m}(a, \mathbf{u})$ as follows:

$$
\begin{aligned}
O_{0}^{t, \mathbf{c}, m}(c) & =1 \\
O_{\tau+1}^{t, \mathbf{c}, m}(b, \mathbf{f}) & =\sum_{(a, \mathbf{u}) \mid k_{\tau}^{m}(a, \mathbf{u}) \geq C}\left(O_{\tau}^{t, \mathbf{c}, m}(a, \mathbf{u}) .\right. \\
\left.P\left[(b, \mathbf{f})_{\tau+1} \mid(a, \mathbf{u})_{\tau}\right]\right), \text { for } \tau \geq 1 &
\end{aligned}
$$

with, $k_{\tau}^{m}(a, \mathbf{u})=a \cdot \mathbf{1}_{\{m-\tau>0\}}+\sum_{r=0}^{\max \{0 ; m-\tau\}}\left(u_{r}\right)$, the sum of all patient types prioritized over the tagged patient with time until due time of $m$, at $\tau$ time slots after arrival. Note that $\mathbf{1}_{\{x\}}$ is set to 1 if $x$ evaluates true, and 0 otherwise, and $\left.P\left[(b, \mathbf{f})_{\tau+1} \mid(a, \mathbf{u})_{\tau}\right]\right)$ are the updated transition probabilities. Similar to the waiting time for scheduled patients, $W_{\tau}^{t, m}(b, \mathbf{f})$ denotes the probability that an unscheduled patient with time until due time of $m$, that arrives in state $(b, \mathbf{f})$ at time $t$, waits for $\tau$ time periods can be calculated by:

$$
W_{\tau}^{t, m}(b, \mathbf{f})=\sum_{(a, \mathbf{u}) \mid k_{t}^{m}(a, \mathbf{u})<C} O_{\tau}^{t, \mathbf{c}, m}(a, \mathbf{u}), \text { where } \mathbf{c}=(b, \mathbf{f})
$$

Table 4.7 Notation introduced in Section 4.7

\begin{tabular}{cl}
\hline symbol & description \\
\hline$O_{t}(a, \mathbf{u})$ & $P[$ Number of scheduled and unscheduled patients in slot $t$ is $(a, \mathbf{u})]$ \\
$O_{t}^{\prime}(a, \mathbf{u})$ & $P[$ Number of scheduled and unscheduled patients in slot $t$, just after \\
& starting diagnostics, is $(a, \mathbf{u})]$ \\
$Q_{t}^{i}(a, \mathbf{u})$ & $P[$ Patient of type $i$ encounters state $(a, \mathbf{u})$ when arriving in slot \\
& $\left.[t-1, t]\left(i=a, u_{0}, \ldots, u_{R}\right)\right]$ \\
$O_{\tau}^{t, \mathbf{c}, i}(a, \mathbf{u})$ & $P\left[\right.$ Patient of type $i,\left(i=a, u_{0}, \ldots, u_{R}\right)$, arriving in state $c(c=b, \mathbf{f})$ \\
& in slot $t$, is in state $(a, \mathbf{u})$ after $\tau$ time slots $]$ \\
$W_{\tau}^{t, i}(b, \mathbf{f})$ & $\begin{array}{l}\mathrm{P}\left[\text { Patient of type } i,\left(i=a, u_{0}, \ldots, u_{R}\right), \text { that arrives in state }(b, \mathbf{f}) \text { in }\right. \\
\text { slot } t \text { waits for } \tau \text { time slots }]\end{array}$ \\
$W_{\tau}^{t, i}$ & $\mathrm{P}\left[\right.$ Patient of type $i,\left(i=a, u_{0}, \ldots, u_{R}\right)$, arriving at time $t$ waits for \\
& $\tau$ time slots $]$ \\
& (minimum $)$ probability constraint that an unscheduled patient is \\
& treated on time
\end{tabular}

To formulate the performance constraint of unscheduled patients, we denote OTP as the probability constraint that unscheduled patients must be treated before their due time. Second, $W_{\tau}^{t, m}$ denotes the probability that an arriving unscheduled patient in slot $t$, with time until due time $m$, regardless of arrival state, waits for $\tau$ slots. The performance can then be calculated by summing the 
probabilities of waiting past due time $m$, these are given respectively by:

$$
\begin{aligned}
W_{\tau}^{t, m} & =\sum_{(b, \mathbf{f})} Q_{t}^{m}(b, \mathbf{f}) \cdot W_{\tau}^{t, m}(b, \mathbf{f}) \\
\sum_{\tau=m+1}^{\infty} W_{\tau}^{t, m} & <1-O T P \forall t, m
\end{aligned}
$$

As we aim to minimize and evenly spread the waiting time of scheduled patients during the day, while ensuring a pre-specified percentage of unscheduled arrivals is seen before their due time, we formulate our objective as follows:

$$
\begin{aligned}
& \underset{t}{\operatorname{minimize}} \max _{t} \mathbb{E}\left[W^{t}\right] \\
& \text { s.t. } \\
& \qquad \sum_{\tau=m+1}^{\infty} W_{\tau}^{t, m}<1-O T P \forall t, m
\end{aligned}
$$





\section{Emergency OR or not: A simulation study of emergency surgery scheduling policies}

\subsection{Introduction}

Hospitals that perform surgery on both elective and emergency patients are faced with the problem of how to deal with arriving emergency patients [238]. In this chapter we study three emergency surgery scheduling policies, commonly encountered both in practice and in the literature. The policies differ in the way emergency patients are allocated to operating rooms (ORs):

1. Reserve ORs for emergency patients (dedicated policy)

2. Treat emergency patients in elective ORs (flexible policy)

3. A mix of the above (hybrid policy)

Policy (1) assigns all emergency patients to dedicated emergency rooms, thus preventing emergency patients from disrupting the elective program. Policy (1) poses a trade-off between operating room utilization and emergency surgery waiting time: having more emergency ORs reduces waiting time, but also reduces utilization. Policy (2) assigns all emergency patients to elective operating rooms. This is done by inserting emergency surgeries into the elective program without preempting elective surgeries. Consequently, an emergency surgery may start in any suitable elective OR as soon as one becomes available, and elective surgeries therefore must be postponed or rescheduled. Furthermore, since preemption is not allowed, emergency patients may have to wait. Policy (3) combines advantages of both policies (1) and (2), emergency patients may be operated in both elective and emergency ORs. If all emergency ORs are occupied and an elective OR is available, emergency patients are treated in this elective OR. In case all ORs are busy, emergency patients that arrive wait and are treated in the first OR that becomes available, regardless of OR type (i.e., elective or emergency OR). This policy aims to reduce the interruptions in the elective patient schedule caused by emergency arrivals, while still allowing timely treatment of 


\section{Chapter 5. Emergency OR or not: A simulation study of emergency surgery scheduling policies}

emergencies if necessary. Hospitals are thus faced with the question which policy to choose, and in case of policy (2) and (3), how many ORs to dedicate to emergency patients.

Use of a specific policy affects a hospital's performance (e.g., utilization, waiting times, overtime). Currently, these effects are not clear from the literature, and there is no agreement on what works 'best'. For example, using a flexible policy (2) is stated to both decrease [253] and increase [68] overtime of ORs. Also, in the literature there are contradictory results between policies [238]. The performance of a used policy is affected by the characteristics of the hospital, and thus differences in hospital characteristics may account for the difference in results between papers. For example, using Policy (2) may only be possible if there are sufficient ORs to ensure a timely start of treatment for emergency patients. Examples of characteristics affecting this performance are: \# of ORs, elective case mix, volume, and elective surgery durations/variability. In addition, in the case of Policy (2) and (3), performance is also affected by the elective surgery scheduling and sequencing policy. Therefore it is beneficial to not only evaluate the differences of a policy for a specific hospital case, but to extend evaluation across hospital characteristics.

Our contribution is that we do not carry out a single case study, but evaluate the different policies under many case (hospital) characteristics. This is done using case mix data gathered from many hospitals, containing information on surgery types and durations, as well as arrival intensities, for both elective and emergency patients. In addition to the hospitals' characteristics, we create and evaluate multiple case characteristic and scheduling policy combinations. This is done using existing OR simulation software, which embeds various algorithms for surgery scheduling. This not only allows to evaluate policies for a specific case, but also evaluate the trends and effects of different characteristics on the policies, which has previously not been done in literature. In addition, we take into account many often encountered performance indicators from literature. Previous studies often use performance indicators relevant for their specific case study which may differ considerably from other studies, making comparisons impossible. We have performed a huge number of simulations, and gathered results in a tool called ORanalyzer. This tool is made available online, along with a manual in this section. The tool allows healthcare practitioners to gain insights into the effects of the scheduling policies in settings similar to their specific hospital setting. In addition, this tool allows others researching emergency scheduling policies to frame their hospital settings in our tool, and compare results. Finally, we present a selection of simulation results. With the tool, the reader can further compare and evaluate scenarios.

The structure of this chapter is as follows. In Section 5.2 we review the literature, and Section 5.3 gives our problem description. Section 5.4 details our approach, and Section 5.5 contains the simulation model and OR analyzer tool. In Section 5.6 we validate our approach, and give results of our research. Finally, Section 5.7 provides a discussion and conclusion. 


\section{$5.2 \quad$ Literature}

There is a considerable amount of literature on operating room planning and scheduling. As the operating rooms (ORs) are often seen as the 'heart' of hospitals it is not surprising that this is a topic of much interest. Literature reviews on (among others) OR planning and scheduling were done by [40], [89], and [114]. We consider an OR planning problem where emergency surgery arrivals have to be taken into account. Work that specifically addresses non-elective surgeries, and takes such surgeries into account on a tactical and/or operational level is less common. Two recent literature reviews that give an overview of OR planning and scheduling literature, taking into account non-electives and the trade-offs they pose, are Ferrand et al. [70] and Van Riet et al.[238]. Van Riet et al. [238] note that it is still unclear what the effect is of different settings on the possible performance measures, and that a key question is how to determine what level of demand and patient mix is required before a specific policy should be pursued. For an extensive overview of the literature we refer to these two papers. Starting from the aforementioned literature reviews we selected studies that evaluated the use of one or more policies to deal with unexpected emergency patient arrivals from an operational research perspective. In addition, we also searched for newer studies not included in the latest literature reviews.

Wullink et al. [253] also look at the use of different policies to treat emergency patients. In their paper they compare the use of dedicated emergency ORs and general ORs, for a case study with in total 12 ORs. Looking at waiting time for emergencies, overtime, and utilization they conclude that general ORs perform better on all performance measures when using a flexible policy. Based on their work the hospital decided to close dedicated ORs. Van Veen-Berkx et al. [239] compare the operating theater performance before and after the dedicated OR was closed in the same hospital and find contradicting results in practice, concluding that overtime increased. It is unclear however, whether other changes took place in between measurements. In addition, overtime is defined differently between studies. Wullink et al. define overtime as the time used for surgeries after regular OR time ends. Van Veen-Berkx et al. define overtime as the difference (in minutes) between scheduled and actual surgery end time of the last patient of the day. Under a flexible policy however, less elective patients are scheduled in ORs (to account for the emergency arrivals), and the end time of the last scheduled patient occurs before the regular closing time of the OR. This difference between scheduled end time and regular OR closing time may account for the conflicting overtime results. Similar work investigating a dedicated and flexible policy is the work by Ferrand et al. [68]. They consider a hospital with 20 operating rooms, and in case of the dedicated OR policy, vary the number of dedicated ORs. They also evaluate waiting time, utilization and overtime. Contrary to the results of Wullink et al. [253], however, the average overtime per day increases under a flexible policy. Similarly, Persson and Persson [201] use simulation to model a swedish orthopeadic department with 2 ORs, and find a dedicated policy out- 


\section{Chapter 5. Emergency OR or not: A simulation study of emergency surgery scheduling policies}

performs a flexible one. However, in their comparison, total available capacity also increased. More recent work by Ferrand et al. [69] builds upon their earlier work [68] and investigates the effect of utilizing some ORs by both elective and emergency patients. Their case study again entails $20 \mathrm{ORs}$, and they vary the number of dedicated and flexible ORs. In addition, they perform a sensitivity analysis to evaluate the effect of increased emergency arrivals, non-stationary (emergency) arrivals, and different procedure times for elective patients. Lans et al. [158] use computer simulation to look at both tactical and operational planning levels when evaluating different policies to anticipate emergency surgeries. On the tactical level they evaluate: (1) dedicated ORs, (2) planned slack in some ORs, and (3) planned slack in all ORs. Performance measures used are utilization, overtime, and waiting time of emergency surgeries. Accounting for all measures they find using planned slack in all ORs performs best. A different policy is evaluated by Bowers and Mould [32] where they investigate the effect of planning elective patients in an orthopaedic emergency OR session using simulation. These patients have a shorter access time, however there is a bigger chance of cancellation. They find that throughput and utilization may increase, as long as elective patients are willing to accept a cancellation probability.

It should be noted that, when using Policy (2) or (3), the way in which elective patients are scheduled may impact performance. Given that surgeries cannot be interrupted, emergency patients can only be treated if an OR is available, and otherwise must 'break in' into the elective schedule when an elective surgery completes, before the next elective surgery starts. These completion times of surgeries are also called 'break-in-moments' (BIMs) [64]. As such, it may be beneficial for emergency patients to ensure that 'break in' moments (BIM) are spread out evenly across the day. Van Essen et al. [64] take this into account and optimize surgery schedules such that the maximum time between BIMs is minimized.

The use of different policies has also been studied in practice. Bhattacharyya et al. [26] report a retrospective analysis where two one year periods were compared before and after the use of a dedicated emergency OR for orthopaedic surgeries. They measured the utilization, cases done during the night (overtime), and the delay frequency of elective surgeries caused by emergencies. They conclude that overtime was considerably reduced by using a dedicated OR. Similarly, Wixted et al. [252] perform a retrospective review after a dedicated trauma OR was opened. They compare performance for a specific surgery type (isolated femur fractures) and find that less overtime and disruptions have taken place since a dedicated OR is used. Noted should be however that during the study several changes occurred such as an increase in surgeons, as well the number of surgeries performed. Sandbaek et al. [214] carry out a before and after analysis of implementing a dedicated policy (from a flexible policy), where three ORs are reserved for emergency patients. They report a reduction in overtime for both elective and emergency ORs, as well as an increase in utilization. However, during this redesign of the ORs a new patient classification and booking policy was 
also introduced, which might effect results.

We note that most research into OR planning so far started from a single case study, with specific hospital characteristics. This may lead to conflicting results, and be of little use to healthcare providers with their own specific case characteristics. The contribution of this research is that we fill this gap by investigating the performance of the different policies, under different case characteristics, and evaluate the circumstances under which specific policies may be best. In addition we create, and make available, a tool such that readers and healthcare practitioners may further evaluate the policies under different hospital characteristics.

\subsection{Problem Description}

In this research, we aim to evaluate and compare different policies that are used in practice to deal with unscheduled emergency arrivals. We do this not for a specific case study, but in a more generalized setting by simulating policies under many case settings, for many performance indicators. As noted in Section 5.1 we investigate the following policies.

1. Dedicated ORs for both elective and emergency patients

2. Treat emergency patients in the elective ORs

\section{A mix of the above}

In Policy (1), one or more OR(s) are reserved for emergency patients, and if emergency patients arrive they are treated in one of the available dedicated ORs. If all emergency ORs are occupied, arriving emergency patients wait and are treated in the first dedicated OR that becomes available. In Policy (2), no ORs are reserved for emergencies. Instead, all ORs are planned with elective patients, and if emergency patients arrive, they will be treated in the first OR that becomes available, delaying the elective patients that are planned in that OR. Policy (3) combines Policy (1) and (2), while some ORs are reserved for emergencies similar to Policy (1). In Policy (3), if all emergency ORs are occupied and an elective OR is available, emergency patients are treated in this elective OR. In case all ORs are busy, emergency patients that arrive wait and are treated in the first OR that becomes available, regardless of OR type (i.e., elective or emergency OR).

To specify the used policy we denote whether emergency patients may be treated in elective ORs, as well as the number of ORs that are dedicated to emergencies. In addition, we evaluate the effect of Break In Moment (BIM) optimization [64], which may effect the performance of Policies (2) and (3) when emergency patients are treated in elective ORs. Combining these variables allows the evaluation of all policies, Table 5.1 shows the variables. Note that the minimum and maximum number of dedicated emergency ORs is based on the number of emergency patients, which is detailed in 5.4.1. 
Table 5.1 input variables.

\begin{tabular}{cl|l}
\hline parameter & description & values \\
\hline EmAnyOR & $\begin{array}{l}\text { Treat emergency patients in elec- } \\
\text { tive ORs }\end{array}$ & $\begin{array}{l}\text { true, false }\} \\
\text { nEmOR }\end{array}$ \\
$\begin{array}{l}\text { Number of ORs reserved for } \\
\text { emergency patients }\end{array}$ & $\begin{array}{l}\{0,1,2, \ldots, 20\}, \text { based on system } \\
\text { load } \\
\text { BIM }\end{array}$ & $\begin{array}{l}\text { Use of Break In Moment Opti- } \\
\text { mization (BIM) }\end{array}$ \\
\hline
\end{tabular}

\subsection{Approach}

In this section we present the approach taken to investigate the different policies defined in Section 5.3. To compare policies not only between each other, but also under different case settings, we define broad hospital settings that make up a specific case setting. All possible case settings are then evaluated under all possible policies, including multiple settings per policy (e.g., Policy 2 having $1,2, .$. emergency ORs) using computer simulation. Section 5.4.1 details the case characteristics that make up the evaluated scenarios, and Section 5.4.2 describes the used performance indicators to evaluate the different policies and scenarios.

\subsubsection{Case characteristics}

As noted in Section 5.2, most studies consider a specific hospital with a specific case mix. The case mix describes the volume and properties of surgery types (i.e., sampled distributions and parameters) that a hospital performs. As the case mix may effect performance, we perform an extensive scenario analysis, including different case mixes. To create distinct case mixes, we use the classification put forward by Leeftink and Hans [162]. They classify surgery types using two parameters: surgery duration (relative to total OR capacity) and coefficient of variation $(\mathrm{CV})$ of the surgery duration, which both indicate the level of complexity involved in scheduling surgeries. Case mixes that include surgeries with a higher duration are more difficult to schedule, as schedules are more likely to have gaps which could not be filled. Conversely, low duration surgeries are easier to schedule, as gaps can be easily filled. The coefficient of variation indicates the variability of surgeries, which in turn leads to more uncertainty when carrying out planned schedules [236]. High variability may lead to overtime and cancellations, and to account for the variability slack may be used when creating schedules [100].

Using these two parameters it is possible to create different case mixes. To create multiple case mixes we use a dataset that is based on historical data from both academic and non-academic hospitals throughout the Netherlands over the past 10 years. In this dataset we categorize surgeries as high/low duration, as well as high/low coefficient of variation. Specifically, we denote a relative mean duration and coefficient of variation smaller than 0.5 as low, and above as high. 
Figure 5.1 shows the case mix of three different hospitals that are included in the dataset. The figures show that case mixes can differ considerably between hospitals: hospital 1 (left) has surgery types with both high and low duration and CV, hospital 2 (mid) almost exclusively has low duration and CV surgery types. Hospital 3 (right) has another case mix, where surgeries have both high and low $\mathrm{CV}$, and only few high duration surgeries are carried out.

Figure 5.1 Case mixes of three Dutch hospitals using classification by Leeftink and Hans [162]
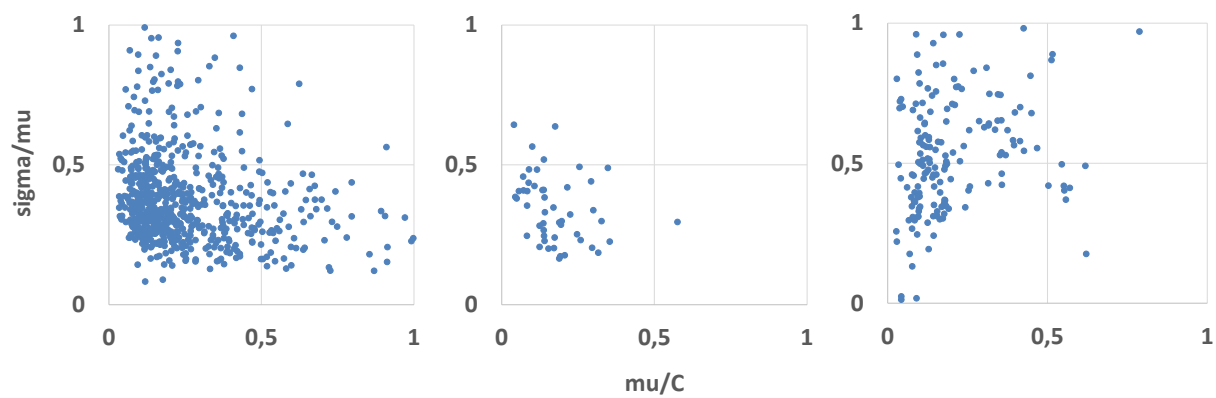

The surgery types underlying the dataset used to construct scenarios and evaluate policies is the same as used by Leeftink and Hans [162]. This dataset contains surgery types, defined as 3-parameter lognormal distributions, which are shown to fit well to surgery duration distributions [178, 222], and denotes the (relative) frequency with which these surgeries take place. Figure 5.2 shows a visualization of the surgeries contained in the dataset, plotted according to surgery duration and coefficient of variation, as well as the categorization of surgeries in high and low duration and coefficient of variation. Specifically, quadrant 1 contains surgeries with a high $\mathrm{CV}$, and low duration, quadrant 2 contains both high $\mathrm{CV}$ and duration surgeries, quadrant 3 both low CV and duration surgeries, and finally quadrant 4 contains low $\mathrm{CV}$ and high duration surgeries. and surgeries are sampled using the case mixes from Table 5.2.

To evaluate the influence surgery type case mixes have on the performance of policies, we sample surgeries from varying quadrant combinations to create theoretical case mixes. For example, by sampling surgeries from quadrant 1 and 3 only, we have a case mix of surgeries with a low (average) duration, and both high and low variability. The theoretical case mixes are detailed in Table 5.2. Note that a case mix may contain surgeries from multiple quadrants.

In addition to case mixes, we vary the number of patients treated (in total) per year, as well as the total number of available ORs. Finally, the percentage of emergency patients is varied, to further specify the case mix, as different hospitals have different numbers of emergency arrivals [238]. Table 5.3 lists the varied case characteristics. Given the varied case characteristics it may be possible that a specific scenario is overloaded. For example, scheduling 20000 patients in only 5 


\section{Chapter 5. Emergency OR or not: A simulation study of emergency surgery scheduling policies}

Figure 5.2 Dataset of surgery types based on historical data of Dutch hospitals categorized by $\mathrm{CV}$ and duration

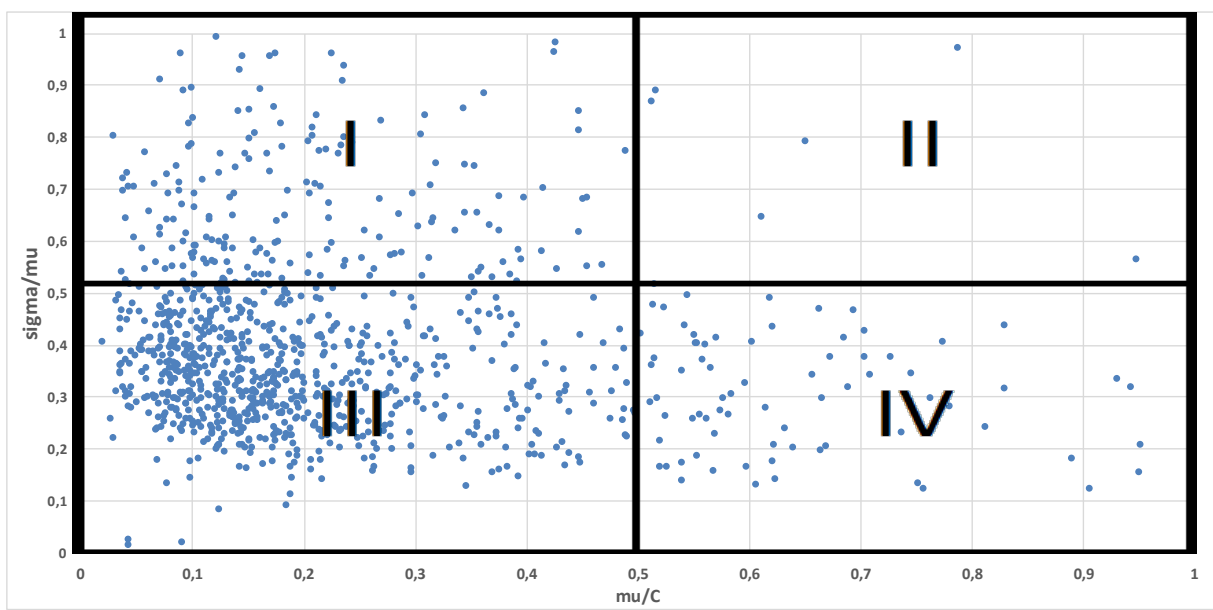

Table 5.2 Evaluated theoretical case mixes

Case Mix Quadrants Included surgery types

\begin{tabular}{ccl} 
A & 1 & low duration; low coefficient of variation \\
$\mathrm{B}$ & 1,3 & low duration; high and low coefficient of variation \\
$\mathrm{C}$ & 3,4 & high and low duration; low coefficient of variation \\
$\mathrm{D}$ & $1,2,3,4$ & high and low duration; high and low coefficient of variation \\
\hline
\end{tabular}

ORs may be impossible regardless of policy, as the required capacity exceeds the total OR time available. Similarly, a scenario may be overcapacitated such that regardless of policy all patients may be treated. We define the load of a scenario as follows:

System load $=$

$\underline{\mathbb{E}[\text { surgery duration }] \cdot \text { Number of patients treated per year }}$

total yearly capacity

We only evaluate scenarios where the load is between $40 \%$ and $100 \%$ (i.e., $0.4 \leq$ system load $\leq 1$ ) to avoid scenarios that are infeasible or irrelevant, and likely not seen in practice. This results in 320.000 different policy and case characteristics combinations to evaluate using computer simulation.

\subsubsection{Performance indicators}

To evaluate the policies we use performance measures most common from recent literature reviews [40, 89, 238], being: utilization, overtime and waiting time (for 
Table 5.3 Varied case characteristics.

\begin{tabular}{cll}
\hline parameter & description & values \\
\hline $\mathrm{CM}$ & case mix of surgery types & \{Case Mix A; Case Mix B; Case Mix C; \\
& & Case Mix D $\}$ \\
nPat & number of patients treated per year & $\{1000,2000, \ldots, 20000\}$ \\
nOR & number of ORs that is available in total & $\{5,6,7, \ldots, 50\}$, based on system load \\
$\% \mathrm{Em}$ & percentage of total patients that is an & $\{0,5,10, \ldots, 40\}$ \\
& emergency patient & \\
\hline
\end{tabular}

both elective and emergency patients). These represent the views of different stakeholders involved within the operating theater, namely patients, staff, and management. With regard to overtime, we evaluate both the average, as well as the percentage of ORs that end in overtime, to evaluate the distribution of overtime over the OR days. In addition, we are interested in outliers with regard to emergency patients (e.g., patients that wait for longer than 30 minutes). While it may acceptable for an emergency patient to wait for some time (e.g., some minutes), waiting a considerable amount of time may reduce health outcomes considerably. Finally, we also evaluate the number of cancelled surgeries (i.e., surgeries that could not start during regular hours). We use the performance indicators listed in Table 5.4.

Table 5.4 Used performance indicators

\begin{tabular}{lll} 
\# & Indicator & Description \\
\hline 1 & Utilization & Percentage of time ORs are in use during regular time \\
2 & PercOT & Percentage of ORs with work in overtime \\
3 & AvrgOT & Average overtime per OR day (in minutes) \\
4 & ElecEW & Average waiting time of elective patients (in minutes) \\
5 & EmergEW & Average waiting time of emergency patients (in minutes) \\
6 & PercEm $>30 \mathrm{~m}$ & $\begin{array}{l}\text { Percentage of emergency patients that wait longer than } 30 \\
\text { minutes }\end{array}$ \\
7 & PercCnld & $\begin{array}{l}\text { Percentage of surgeries that are cancelled due to time con- } \\
\text { straints }\end{array}$ \\
&
\end{tabular}

\subsection{Simulation model and OR Analyzer tool}

In this section we first describe the used simulation model, as well as settings used within the model (5.5.1). Second, we present the OR Analyzer tool that incorporates all results gained from the simulation model to quickly present and evaluate multiple policies and case settings (5.5.2).

\subsubsection{Simulation model}

All simulations are done using Discrete Event simulation (DES) with the 'Operating Room Manager' software [100, 101]. This software is developed using the 


\section{Chapter 5. Emergency OR or not: A simulation study of emergency surgery scheduling policies}

Delphi programming language from CodeGear and allows for the strategic, tactical, and operational evaluation of an operating theater. Within the program, detailed operating theater reconstructions are possible, taking into account additional resources (e.g., diagnostic equipment), and constraints (e.g., scheduled lunch breaks).

In addition, the program incorporates various algorithms, that allow for optimization of the surgery schedule, and a user interface that allows quick changing and re-evaluation of OR settings. Figure 5.3 shows a screenshot of the program.

Figure 5.3 Screenshot of the Simulation model

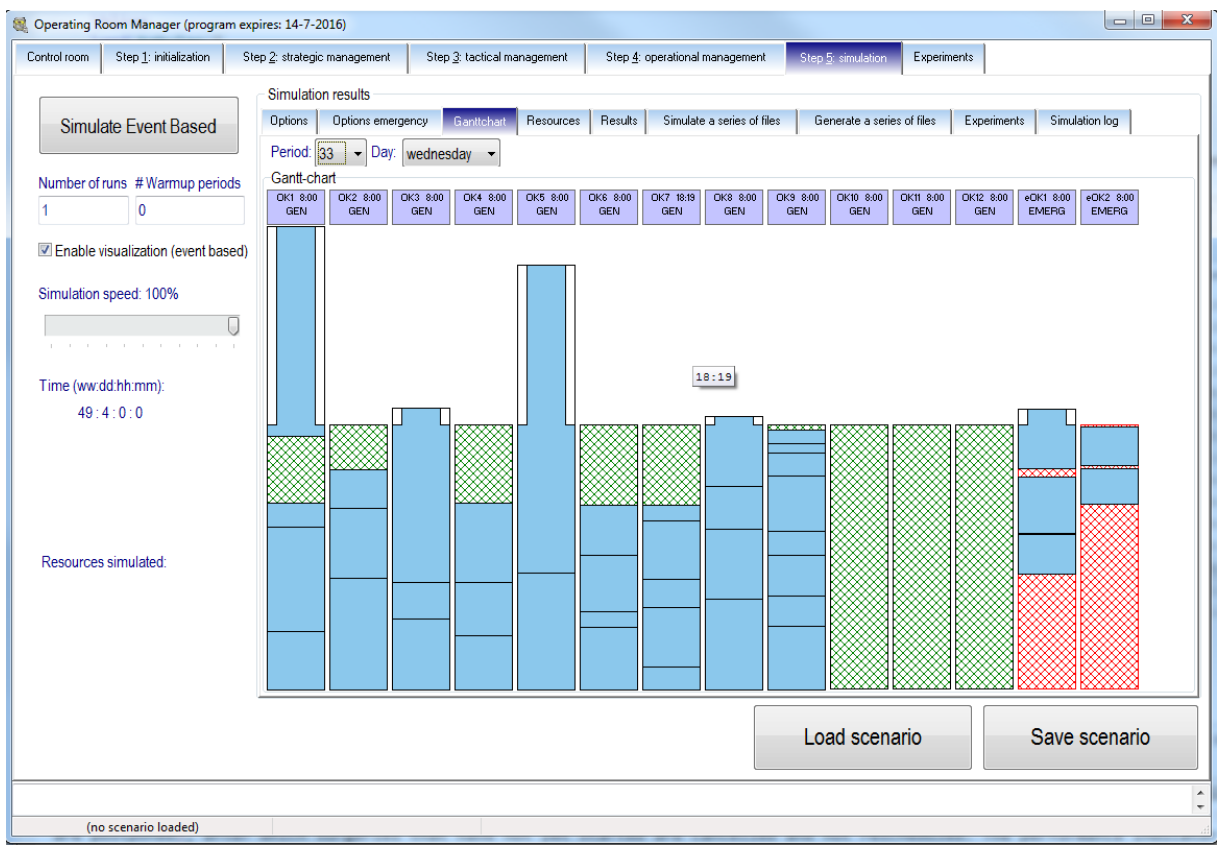

In order to gain insights into the effects of the scheduling policies under different hospital characteristics, we do not model all complexities of a realistic operating theater. First, we assume the number of available (emergency) ORs is the only limiting resource. In practice, some resources besides the ORs may dictate when surgery can start, such as available diagnostic equipment or surgical tools. Similarly, staff may not be available to immediately start a surgery. In the simulation model these resources are not taken into account. Second, setup and change-over times are not considered. In practice, two surgical procedures may require change-over times between them, giving further importance to the elective patient schedule. We assume such changeover times are included in the surgery times. Also, we assume patient flow into and out of ORs may take place immediately (e.g., there are beds available at wards and pre-/post aneastheric 
care units) as we want to exclude effects other departments may have on the performance of the OR.

To evaluate a scenario and policy, we simulate an operating theater for 50 weeks (each week containing 5 working days), with daily capacity of an OR being eight hours. The elective surgeries that are sampled are spread evenly over the weeks (e.g., 5000 elective patients results in 100 patients to schedule per week). When creating the surgical schedules, surgeries are randomly selected and scheduled using a level fit policy, such that workload is spread over all available ORs. When using BIM optimization, surgeries are swapped (within their allocated ORs), such that the maximum Break In Interval is minimized over all ORs. ORs remain operational until 12 AM (i.e., there is no cap on overtime, after which surgeries are postponed), however surgeries that have not started during regular hours are cancelled and not rescheduled. The performance indicator PercCnld indicates how often cancellations take place given a scenario and policy.

Emergency patients that arrive must be treated as soon as possible, and are prioritized by their waiting time. Based on the policy these patients are treated in the first available (elective or emergency) OR, and are always prioritized over elective patients. When allocating patients to ORs we do not specifically plan slack time. Given the evaluated scenario all elective patients are scheduled, and (if any) remaining available OR time is left, this is then spread evenly across ORs, as patients are scheduled using a level fit policy. In addition, we assume all elective surgeries are available at the beginning of the day (i.e., can start as soon as possible), and that there are no no-shows of patients.

Finally, we do not consider possible costs of changing ORs between a flexible and elective setup. In reality, an OR in which both elective and emergency patients may be operated may be more costly, as more patient types must be accommodated. As the costs for such changes would differ between hospitals, decisions including costs are best made by healthcare providers themselves.

Emergency patients are drawn from the same case mix underlying the elective surgeries (as noted in Section 5.4.1), and arrive at the ORs following a poisson process. The (inter)arrival rates for each patient type are chosen such that the (expected) total number of arrivals corresponds with the number of patients arriving per year, as detailed in Table 5.3. For example, with 10000 patients in total per year, of which $10 \%$ are emergency patients, there are 9000 elective patients to schedule, and (on average) 1000 emergency patients arrive during the year, with an overall rate parameter $\lambda$ of $\frac{1000}{2080} \approx 0.48$ per hour (assuming 52 weeks with 5 working days of 8 hours).

\subsubsection{OR Analyzer tool}

The OR Analyzer tool allows quick evaluation and comparison of two scenarios that were simulated using the simulation model, and allows healthcare practitioners to quickly gain insights into the effectiveness of the different policies for their specific hospital case. In addition, insights can be gained with regards to 


\section{Chapter 5. Emergency OR or not: A simulation study of emergency surgery scheduling policies}

"what-if" analyses, such as the effectiveness of policies under expected future patient increases. The tool was developed using the Tableau visualization software package [223]. The tool contains all simulation results and can aggregate, display and visualize the various performance indicators. Figure 5.4 shows a screenshot of the program. At the top a choice can be made between an introduction screen and screens for different stakeholders. In the stakeholder screens, their respective performance indicators are shown. The right columns can be used to set various scenario and policy settings (e.g., number of patients, case mix, etc). This may be done for at most two different scenarios (one column per scenario) simultaneously, which are both shown in the results graph.

Figure 5.4 Screenshot OR analyzer

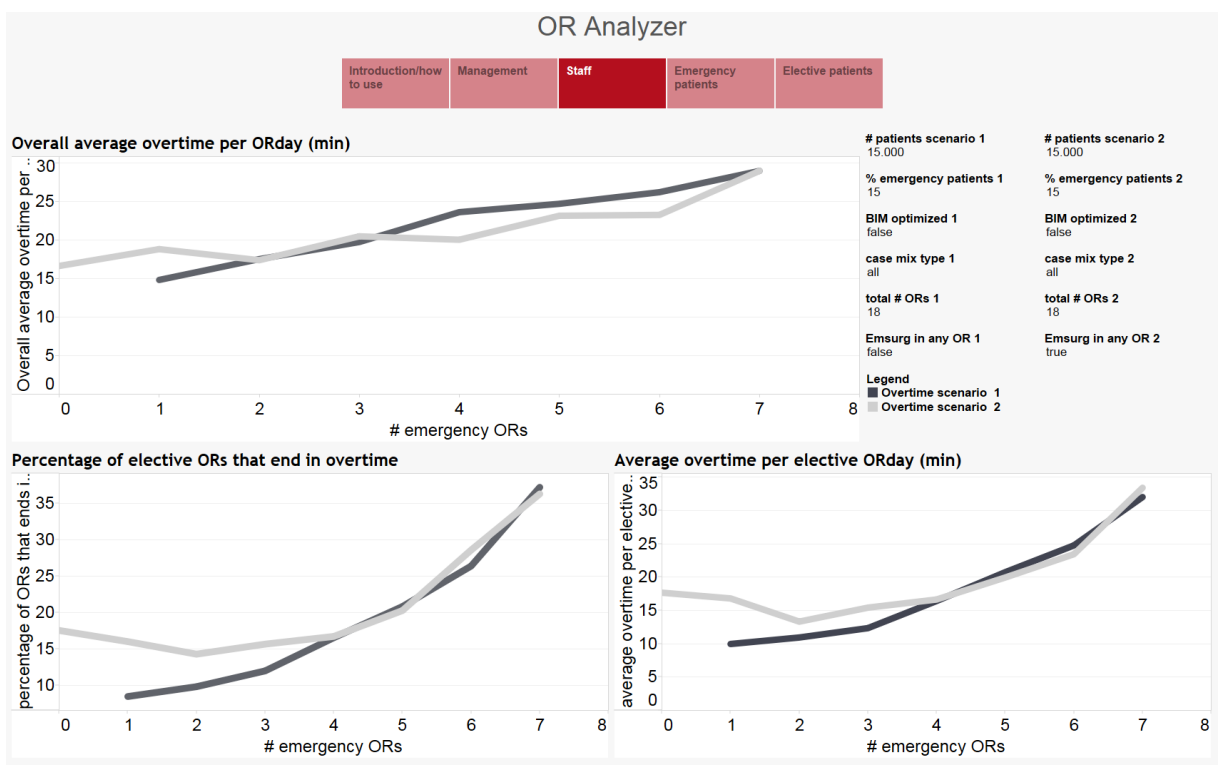

Each performance indicator graph displays its respective performance on the $y$-axis, for a different number of dedicated emergency servers displayed on the $\mathrm{x}$-axis. This allows the user to quickly compare different policies in multiple scenarios. For example, the figure shows all three policies for a hospital case with 18 ORs in total, 15.000 patients, of which 15\% are emergencies; surgeries are drawn from case mix D and there is no BIM optimization carried out. For scenario 1, "Emsurg in any OR" is set to false, indicating emergency patients may only be treated in dedicated ORs (Policy 1), and in scenario 2 it is set to true, allowing emergency patients to be treated anywhere. Scenario 1 shows the overtime performance indicators using a dedicated policy when there are one to six emergency rooms available, and scenario 2 shows performance for the situation that emergencies must be treated in regular ORs (0 emergency ORs; Policy 2), and breaking into the elective program whilst also having emergency ORs (one or more emergency ORs; Policy 3). The figure shows the overtime performance 
indicators for an example setting in which Policy 1 (dark grey), as well as Policies 2 and 3 (light grey) are shown. As expected, both the percentage of elective ORs that end in overtime, as well as average elective overtime per day is higher under Policy 2 or 3 when there are 3 or less dedicated emergency ORs. This is caused by emergency patients also being treated in regular ORs, causing additional delays for electives. In this example case, once there are 4 or more dedicated emergency ORs there is no distinction between Policy 1 and 3 with regards to overtime as there are enough emergency ORs to treat most emergency patients in. We further discuss results in Section 5.6. The OR analyzer is available online (available at: http://tabsoft.co/29jrDwM).

\subsection{Experiments and Results}

In this section we first validate our analyzer using a case study in Section 5.6.1. This is done by first using the simulation model to evaluate and validate a detailed case setting using historical data, and then comparing the detailed simulation outcomes with results using similar case mix options available in the OR analyzer. Second, we compare our findings with some of the results found in the literature that have contradicting outcomes. Finally, we evaluate the performance of the three policies under a wide variety of different settings.

\subsubsection{Validation}

To validate our simulations we compare the analyzer outcomes with a case study from Haga. In this case study we use the historical daily (elective) patient arrivals, as well as the historical emergency arrival rates and case mix, resulting in the hospital case mix shown in Figure 5.5. In addition, we use the historically available ORs as input for the simulation model.

The mean surgery duration of the hospital case mix is 86.4 minutes, which corresponds most with case mix B (mean duration: 89.37). Similarly, most surgeries fall below the $0.5 \mathrm{mu} / \mathrm{C}$ threshold and there are both high and low variability surgeries. There are 9176 patients treated, of which $14,27 \%$ are emergency patients. Finally, there are on average 10.1 ORs available. The hospital uses the hybrid Policy (3), where one dedicated OR is reserved for emergencies, and if necessary break ins take place in the elective ORs.

We validated the simulation model by comparing historical results with those obtained from the model. To this end we simulated our case study, taking into account daily availability of ORs, as well as additional effects such as changeover times between surgeries and different scheduling policies to best represent practice. The case study simulation shows a similar utilization and overtime as historical data. Within the case study, both Policy 2 and 3 (one emergency OR) perform best over all performance indicators. If breaking in is not allowed, it is impossible to have enough emergency ORs for emergency patients, while still having enough elective ORs to schedule all elective patients. 


\section{Chapter 5. Emergency OR or not: A simulation study of emergency surgery scheduling policies}

Figure 5.5 Case mix used for validation, $2015(\mathrm{n}=9176, \mathrm{C}=510$ (minutes) )

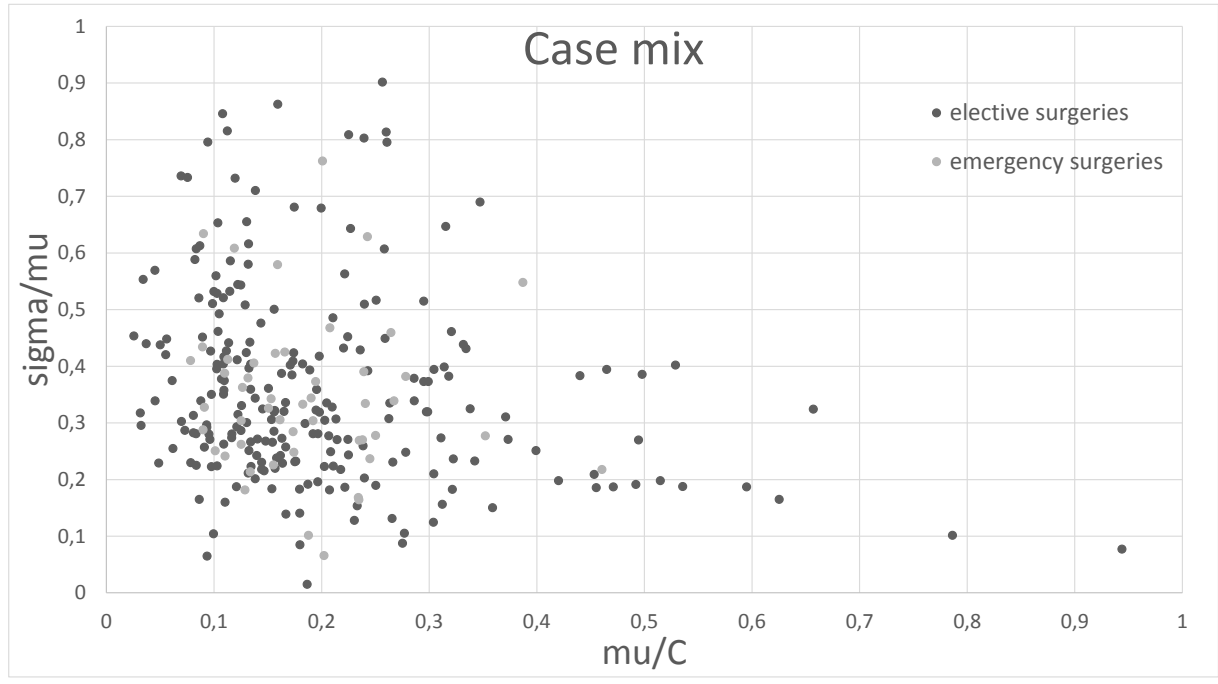

In the analyzer we evaluate the setting most similar to the case study, which is: 9000 patients, 10 ORs, $15 \%$ emergencies, no BIM optimization, and case mix B. Similar to the more detailed simulation we see that using Policy 1 is unable to perform well for both elective and emergency patients. Under a dedicated policy (1) at least three emergency ORs are needed to start emergency surgeries in a timely manner, however around $4 \%$ of elective surgeries are cancelled. In addition, we see that Policy (3) performs best with regards to elective and emergency patient waiting time, with an increase in overtime, similar to the detailed case. From this we see that the generalization from using the analyzer does not yield different conclusions, and it is useful to gain insights for more specific hospital cases.

As noted in van Riet et al. [238], there are conflicting outcomes in the literature, where Wullink et al. [253] states that overtime decreases under Policy 2, but Ferrand et al. [69] states overtime increases under Policy 2. Besides case study differences, there are 12 and 20 ORs respectively, as well as different case mixes, the arrival processes for elective patients differ which max explain the different conclusions. Ferrand aims to schedule elective patients evenly throughout the day, and as such lets a fixed number of elective patients arrive at set intervals throughout the day, based on historical data. In contrast, in Wullink (and our) study elective patients are assumed to be available at the start of the day. The different arrival process makes it difficult to compare outcomes, however when comparing emergency and elective patient waiting times, as well as overtime in a similar size setting in our OR Analyzer we see that, similar to Ferrand, Policy 3 performs best with trends similar to the evaluated case in Section 5.6.2. It is also difficult to compare the results of Wullink with our analyzer, as not all case characteristics are specified, and only the number of ORs, the surgery 
type mix, and number of emergency arrivals per day is given. Wullink concludes that using Policy 2 may increase utilization, reduce waiting time for emergency patients, and reduce overtime. We see similar results for the first two indicators, with elective OR utilization slightly higher when not using dedicated ORs, and an average waiting time for emergency patients decrease. Finally, the average overtime per OR day is (slightly) higher under the break in policy, which differs from their results. However, when using Policy 1, $28 \%$ of emergency patients are cancelled, as their surgery could not start during regular hours. If these patients would be treated, their entire surgery would take place in overtime, and taking this into account we similarly see that overtime is higher under Policy 1.

\subsubsection{The effect of scale}

In smaller cases (i.e., few operating rooms) we expect that Policy 1 performs better, as emergency patients that have to break in most likely have to wait for one of the few surgeries to finish. For smaller cases we expect the opposite, and Policy 2 to perform better as breaking in becomes easier. We now first evaluate the policies for a smaller case setting, followed by a larger setting, to evaluate the effects of OR scale on the policies.

We evaluated the case for 5000 patients, of which $15 \%$ are emergency patients, and 5 available ORs (emergency or elective). The surgeries are sampled from case mix A, and there is no BIM optimization applied. The overall load of the system is approximately $57 \%$. To evaluate the policies, scenario 1 shows the performance of the KPIs, for different numbers of emergency ORs while breaking in is allowed, and scenario 2 while breaking in is not allowed. As such, scenario 1 shows dedicated emergency OR settings (Policy 1), and scenario 2 shows Policy 2 when there are no emergency ORs, and various settings of the hybrid policy (Policy 3). We now discuss the the KPIs for the smaller case for the different stakeholders.

In this small case, displayed in Figure 5.6, up to two dedicated emergency ORs (out of five total) are opened, and in case of scenario 1, breaking in into elective ORs is also allowed. For Policy 2, the average utilization over all ORs is just over 55\%, which then increases up to $80 \%$ under Policy 1 and 3 . When using emergency ORs, their utilization under Policy 3 is lower than for Policy 1, which could be expected, as whenever the emergency ORs are full and an elective OR becomes available, the emergency patient will break in.

Figure 5.7 shows the emergency patient KPIs. It shows that when using Policy 1 , a single dedicated emergency OR is not enough, as the average waiting time is over 15 minutes, over 100 patients wait longer than 30 minutes, and over $6 \%$ of emergency patients could not start surgery during regular hours. However, when two emergency ORs are used in Policy 1, the performance of emergency patient KPIs are better than Policy 2 in terms of average waiting time, and number of times that patients wait more than 30 minutes. In this specific setting, using (sufficient) dedicated emergency ORs is thus preferred over breaking into the elective program. 


\section{Chapter 5. Emergency OR or not: A simulation study of emergency surgery scheduling policies}

Figure 5.6 Average utilization of elective and emergency ORs (min) under Policy 1 (light), Policy 2 (dark, 0 emORs) and Policy 3 (dark, $>0$ emORs), for 5 ORs

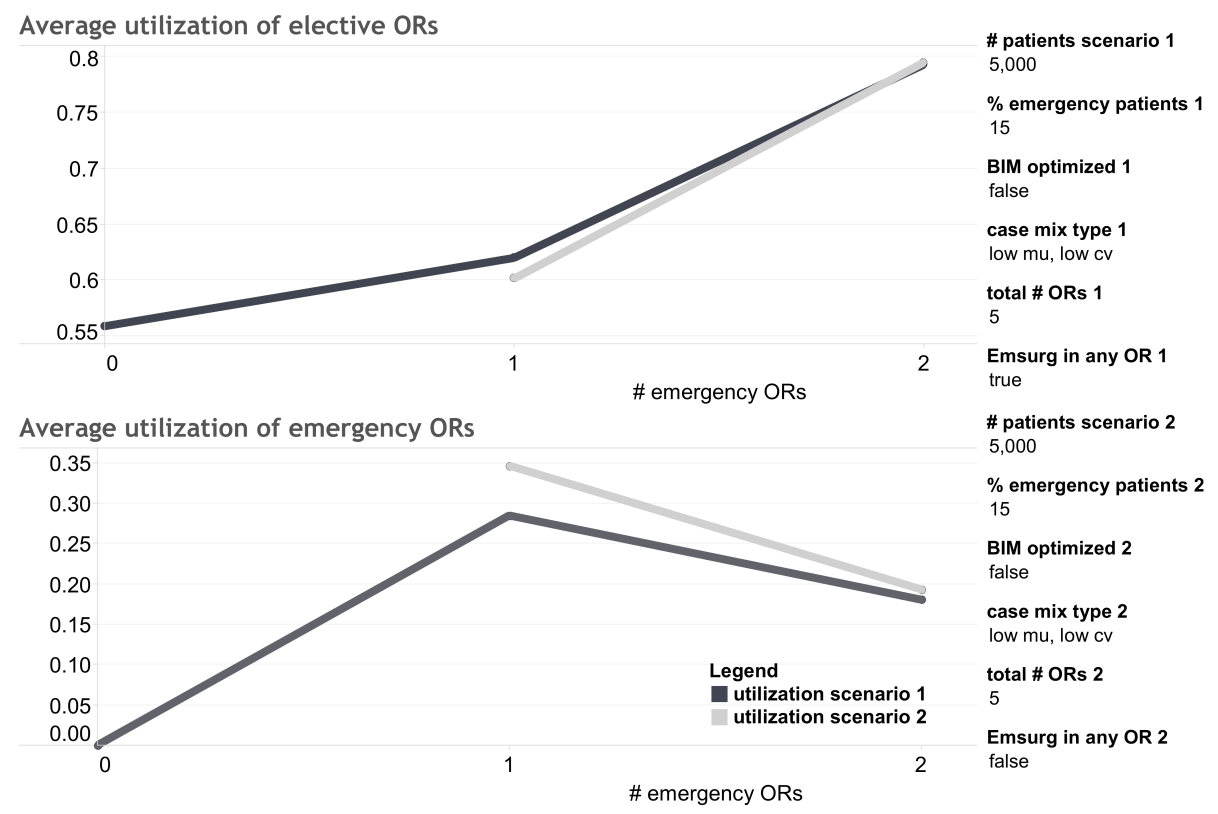

Figure 5.8 shows the elective patient performance indicators. Part of the waiting time of elective patients consists of delays caused by previous (elective) surgeries that finished late. As such, when fewer elective ORs are available, more patients are scheduled per OR, and average waiting times increase. This effect is seen in scenario 2, where opening an additional emergency OR (and thus closing an elective OR), increases average waiting time from under 9 minutes to 11 minutes. However, when there are no emergency ORs, and emergency patients must break in (Policy 2), waiting times for elective patients exceed those of Policy 1, with the average waiting time for elective patients just over 13 minutes, meaning that on average (elective) patients wait 2 minutes longer because of emergency break-ins (2 emORs). Using a hybrid policy however, with 1 dedicated emergency OR, and allowing break ins has the lowest average waiting time for elective patients at around 10 minutes.

Overtime results are displayed in Figure 5.9. The average overtime per OR day is similar for Policy 1 and 2, being just over and under 7 minutes respectively. However, when evaluating the overtime of elective ORs only, the average overtime is 6 minutes when allowing break-ins, and 4 minutes when having dedicated ORs. As one would expect, allowing break-ins increases overtime, albeit in this setting this increase is negligible. In the evaluated small case, Policy 1 seems 
Figure 5.7 Emergency patient performance indicators under Policy 1 (light), Policy 2 (dark, 0 emORs) and Policy 3 (dark, >0 emORs), for 5 ORs
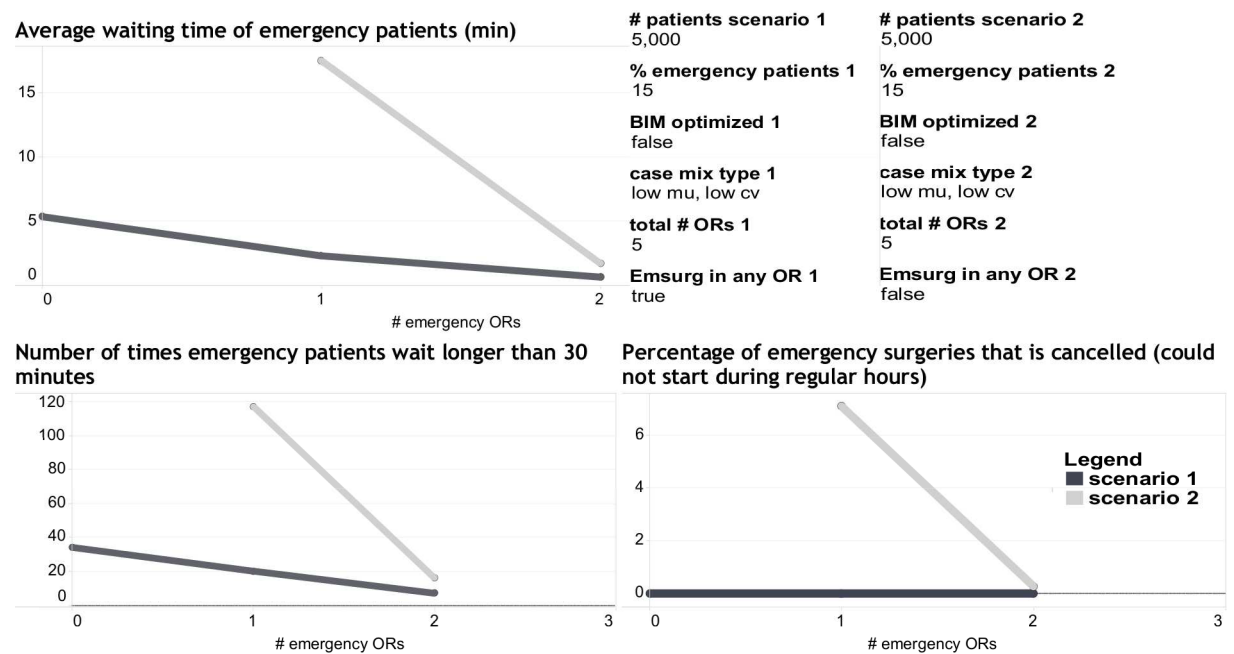

to outperform Policy 2. The waiting time for emergency patients, as well as number of patients that wait over 30 minutes is lower under Policy 1, and at the same time the waiting time of elective patients is lower as well. Finally, there is considerably less overtime when using Policy 1. A hybrid policy however, of using one emergency OR, and allowing break-ins, has similar performance, with less overtime, and lower elective waiting times. When there are only three elective ORs, it becomes more difficult to plan all elective patients, reflected by the utilization of around $80 \%$. When using the hybrid policy, initial capacity for elective patients increases by $33 \%$ (1 OR), while emergencies now break-in when the emergency OR is utilized. Herein, the capacity reduction of emergency break-ins is less than the initial capacity gain, resulting in better performance of the hybrid policy.

To evaluate how the policies perform for different case sizes, we similarly evaluate performance for a larger case setting. Herein there are 15000 patients, of which $15 \%$ are emergencies, and there are 15 ORs in total. Similar to the smaller case mix, this system has a theoretical load of $57 \%$. Aside from the increase in volume and size, the same case mix is used, and there is no breakin moment optimization. In this large case, up to seven dedicated emergency ORs are opened, and in case of scenario 1, breaking in into elective ORs is also allowed. Figure 5.10 shows that initially utilization levels differ between policies, however as more emergency rooms are opened, the difference between dedicated emergency ORs and a hybrid policy disappears and utilization levels become similar. When using Policy 2 or 3, the overall utilization of ORs is around $56 \%$, similar to the smaller case, as the loads are identical.

Figure 5.11 displays the emergency patient KPIs, and we see contrary to the smaller case that, when using Policy 2 or 3 , the average waiting time for 


\section{Chapter 5. Emergency OR or not: A simulation study of emergency surgery scheduling policies}

Figure 5.8 Elective patient performance indicators under Policy 1 (light), Policy 2 (dark, 0 emORs) and Policy 3 (dark, $>0$ emORs), for 5 ORs

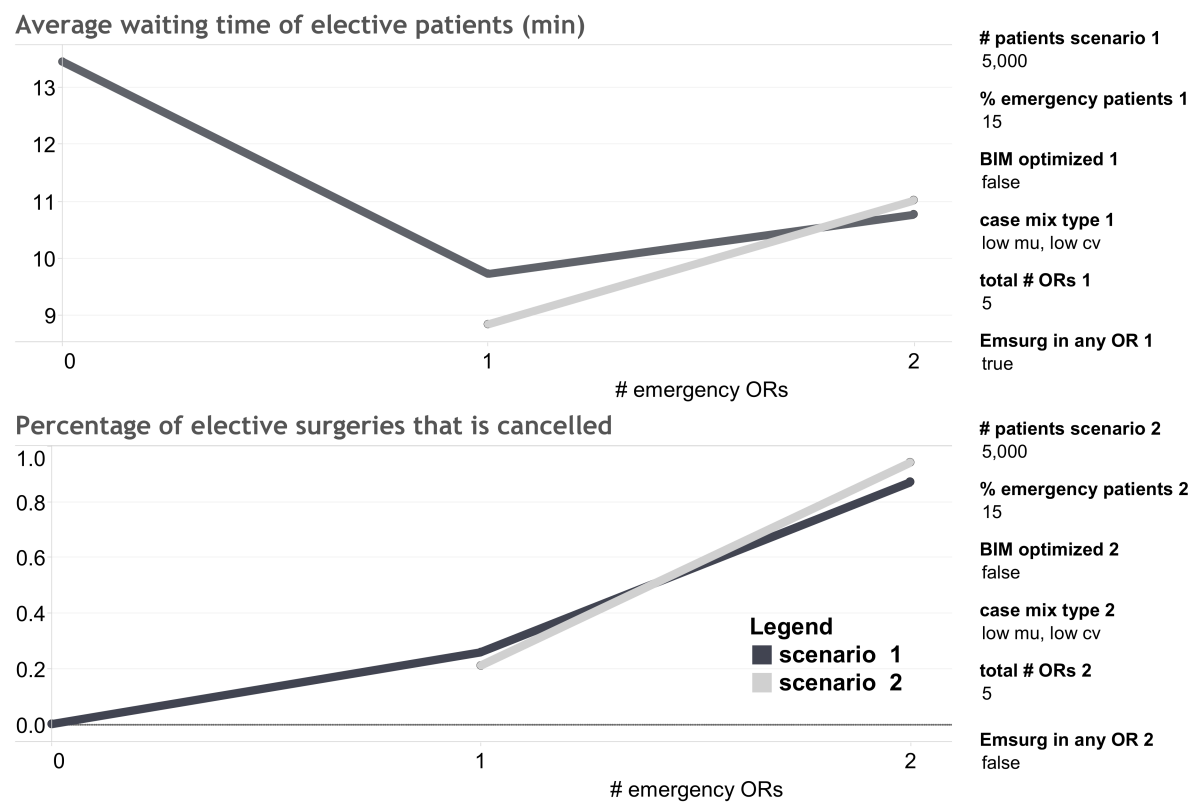

emergency patients is 2 minutes, and drops below 1 minute when there is also a dedicated emergency OR. As expected, when there are this many ORs, emergencies almost never have to wait, as there are many opportunities to break-in during the day. Also, when allowing break-ins, all emergency surgeries are able to start during regular hours, and almost never wait longer than 30 minutes. When using dedicated ORs only however, we see that a certain number of ORs must be dedicated to emergencies before average waiting times and number of emergency patients that wait over 30 minutes drop low enough. The minimum number of emergency ORs that gives less than 10 minutes (average) waiting time for emergencies in Policy 1 is 3. However, in this setting there are still 94 patients that have a waiting time over 30 minutes $(4.2 \%)$ and $1 \%$ of emergencies is unable to start during regular hours.

The average waiting time of elective patients is 9.2 minutes under Policy 1 (3 emORs), and 13.9 minutes under Policy 2. For three or more dedicated emergency ORs, there is no difference between using a dedicated or hybrid policy, as there are sufficient emergency ORs to treat all patients. However, when there are more than 4 emergency ORs, the cancellation percentage of elective patients begins to rapidly increase, indicating that there is no longer enough capacity available to treat all elective patients. When using a hybrid policy with fewer 
Figure 5.9 Overtime performance indicators under Policy 1 (light), Policy 2 (dark, 0 emORs) and Policy 3 (dark, $>0$ emORs), for 5 ORs
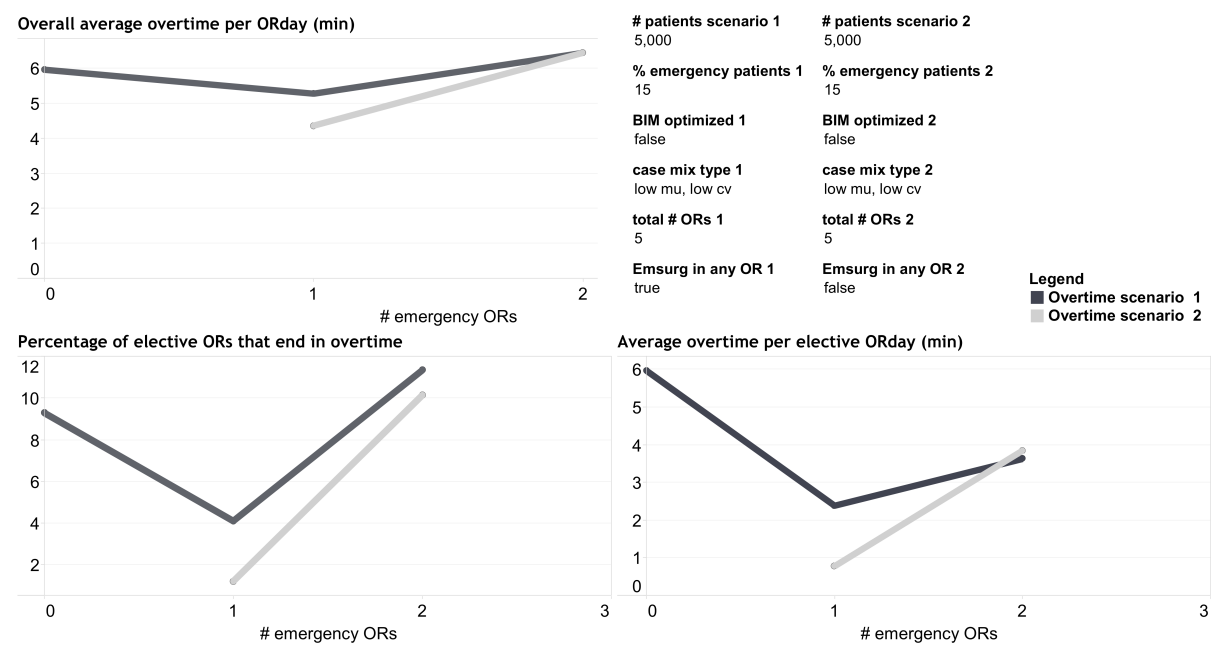

Figure 5.10 Average utilization of elective and emergency ORs (min) under Policy 1 (light), Policy 2 (dark, 0 emORs) and Policy 3 (dark, >0 emORs), for 15 ORs

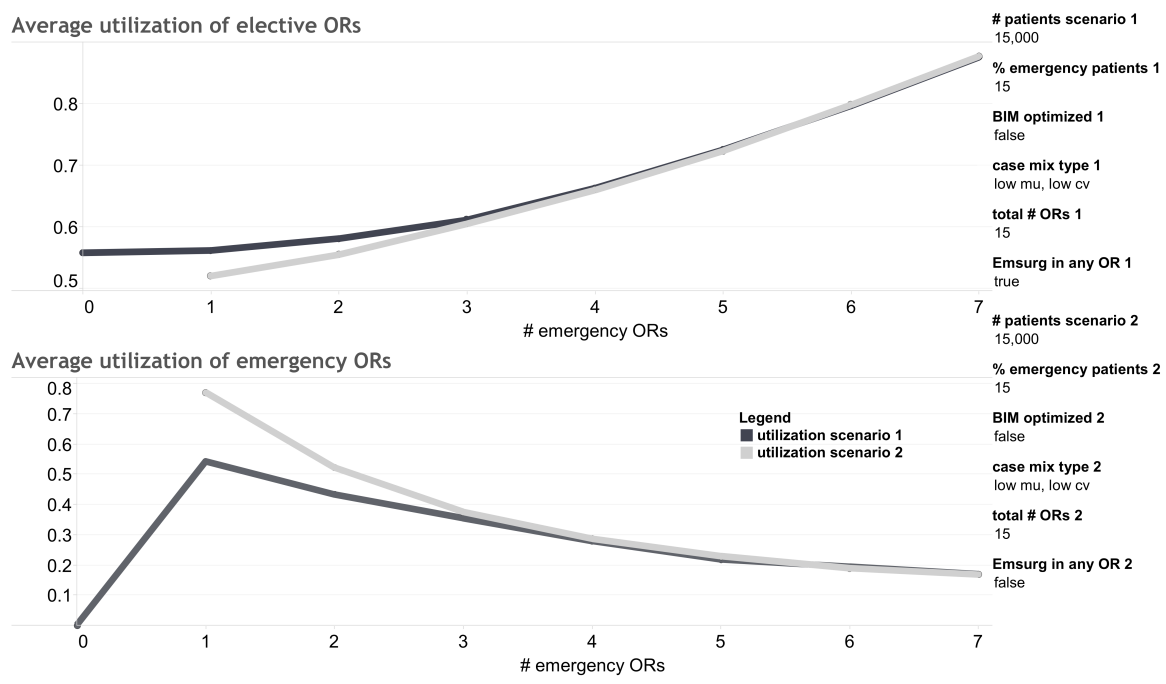

$(<3)$ emORs, the waiting time of elective patients drops, as more emergency patients are treated in an elective OR. Here, a similar effect can be seen as in the smaller case when adding an emergency OR to a hybrid policy, the reduction of interruptions caused by emergencies is greater that the capacity loss of having another emergency OR, up to a certain point, when elective patients can no longer be scheduled. 


\section{Chapter 5. Emergency OR or not: A simulation study of emergency surgery scheduling policies}

Figure 5.11 Emergency patient performance indicators under Policy 1 (light), Policy 2 (dark, 0 emORs) and Policy 3 (dark, $>0$ emORs), for 15 ORs
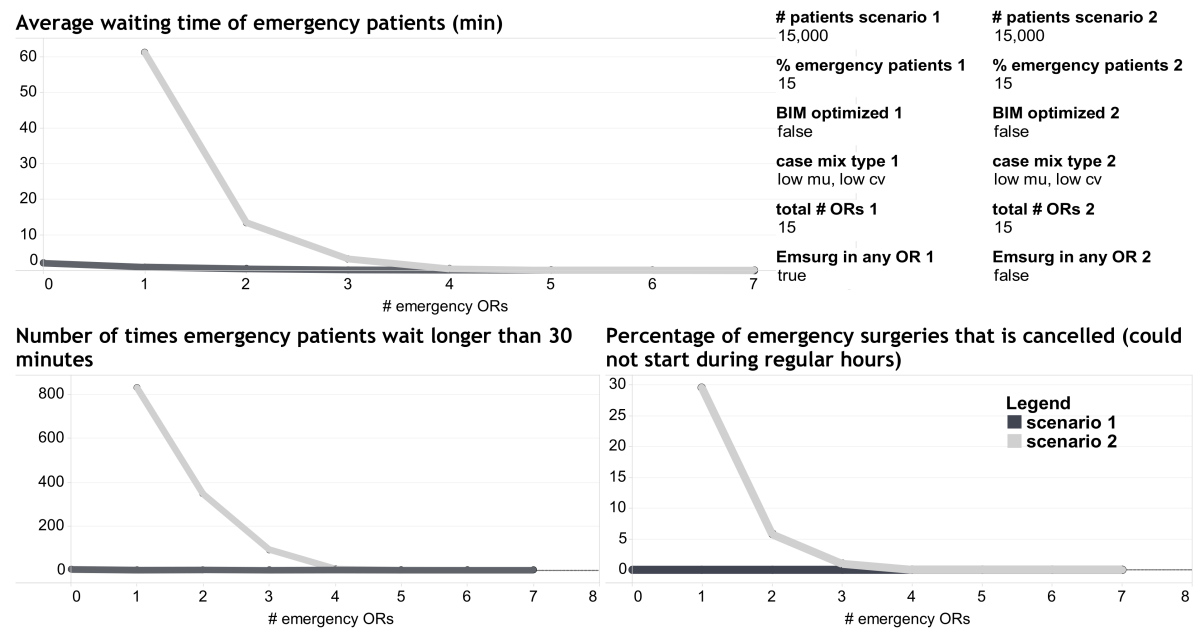

Figure 5.12 Elective patient performance indicators under Policy 1 (light), Policy 2 (dark, 0 emORs) and Policy 3 (dark, >0 emORs), for 15 ORs
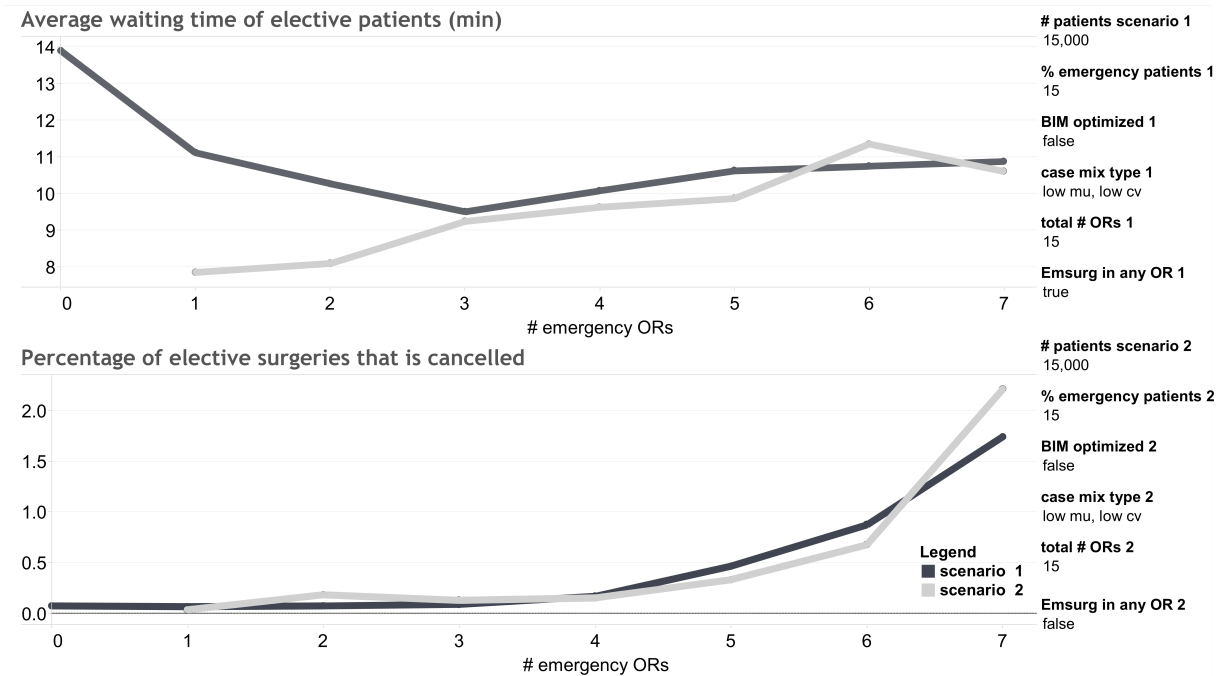

The overtime results are similar to the smaller case. Over all ORs, the overtime averages are roughly the same, however there is considerably more overtime in elective ORs when using Policy 2 (5 minutes), compared to Policy 1 (0.72 minutes). This difference in overtime is caused by the dedicated emergency ORs where under Policy 1 almost all overtime is made. However, to a certain ex- 
tent overtime in emergency ORs may be more acceptable, as this is inherent in treating emergency patients.

Figure 5.13 Overtime performance indicators under Policy 1 (light), Policy 2 (dark, 0 emORs) and Policy 3 (dark, >0 emORs), for 15 ORs

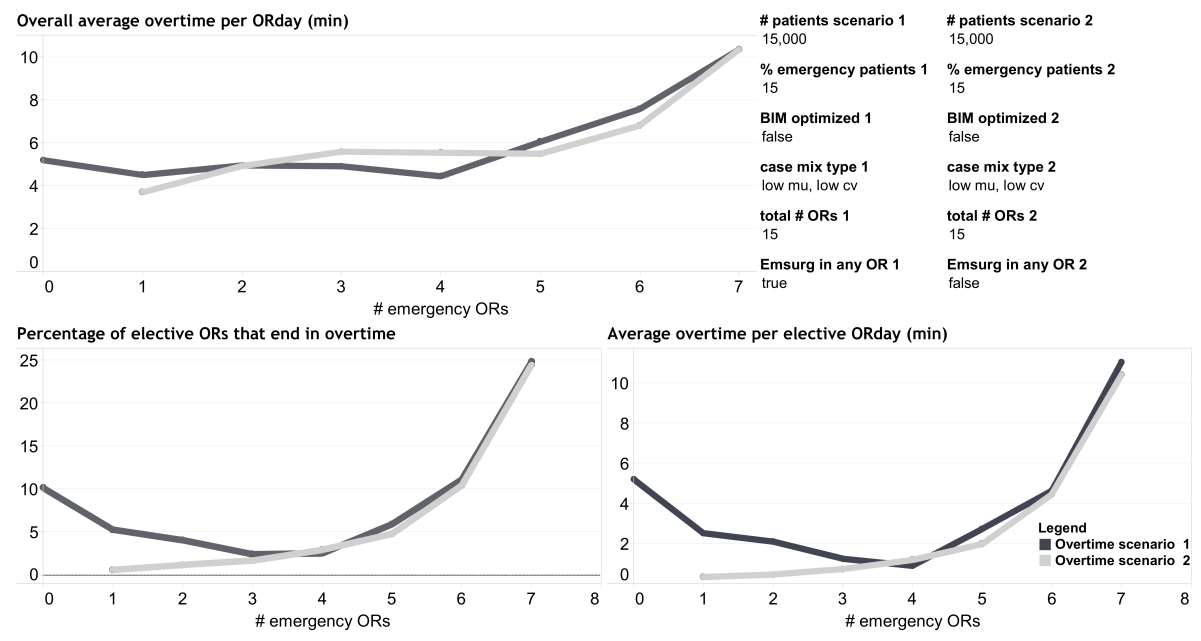

When comparing the small and large case, two findings stand out. First, in the smaller case the use of Policy 2 may not be sufficient. While the average waiting time for emergency patients is around 5 minutes, more than $5 \%$ of patients wait longer than 30 minutes, which is undesirable for emergency patients. In addition, elective patients have longer waiting times in Policy 2, and thus Policy 1 with a proper amount of emergency ORs performs better. In the larger case, using Policy 2 immediately outperforms Policy 1 with regards to emergency patient performance for lower numbers of dedicated emergency rooms. This comes at a cost however, as the average waiting time for elective patients is higher, and the overtime is redistributed across all ORs, meaning staff has more uncertainty with regards to working late. Under Policy 1, you very likely work late when working at an emergency OR, and otherwise not. From this, we see that for smaller operating theaters, using dedicated ORs is more beneficial, and when volume increases, the trade-off becomes less distinct, as breaking in increases emergency patient KPIs, while decreasing elective patient and overtime KPIs. In our cases we found this tipping point to lie at 9 ORs (with a corresponding 9000 patients in total), after which Policy 2 becomes viable for emergency patients. Arguably, other factors besides scale may influence the effectiveness of the used policy such as the case mix, emergency percentage, or overall system load. Regardless of factors, we find that around 8 to 9 ORs Policy 2 becomes feasible, indicating that the total number of ORs is a large determinant of break in policy effectiveness.

Secondly, we see that in both cases, using a hybrid policy may be more beneficial that strictly having break-ins or emergency ORs. This makes sense as it 


\section{Chapter 5. Emergency OR or not: A simulation study of emergency surgery scheduling policies}

poses an intermediary solution between the other two policies, and allows for a better fine tuning for a specific case. In practice, when having dedicated ORs, a certain number is needed to ensure that arriving emergencies dont have to wait too long for the emergency OR to become empty, and as a result, it may be that there is not enough capacity left for the elective patients. When using a hybrid policy, the emergency ORs ensure that there are not too many break-ins in the regular program, reducing the elective waiting time. At the same time however, if it happens that multiple emergency patients arrive in quick succession, there can still be break-ins in the elective program, reducing emergency waiting times. This flexibility allows a better use of the ORs, as effectively a dedicated policy is used, until there are too many emergency arrivals, at which point a break-in policy is used.

Besides the effects of scale on policy effectiveness we also evaluate the effect of variability and overall load. We see that these have a much larger (negative) effect on Policy 1. Figure 5.14 shows an OR setting similar to the larger case of Section 5.6.2 in size (15 ORs), overall load (59\%), and emergencies (15\%), using the surgery types of case mix B with higher variability. Similar to case mix A, all policies are viable (with an appropriate amount of emergency ORs). However, while the expected waiting time for elective patients, as well as cancellation rates have increased for the policies, they increase considerably more for the dedicated policy. Under Policy 2 the average waiting time increased from approximately 14 to 16 minutes, and the cancellation rate from $0 \%$ to $0.62 \%$, while Policy 1 increased from 9.5 minutes to 13.4 minutes, and the cancellation rate from $0.1 \%$ to $1.62 \%$. This effect is further increased as the overall load gets higher. This indicates that, when variability increases, using a break in Policy (2-3) may be more effective in handling emergency surgeries as capacity is better used.

Figure 5.14 Patient performance indicators
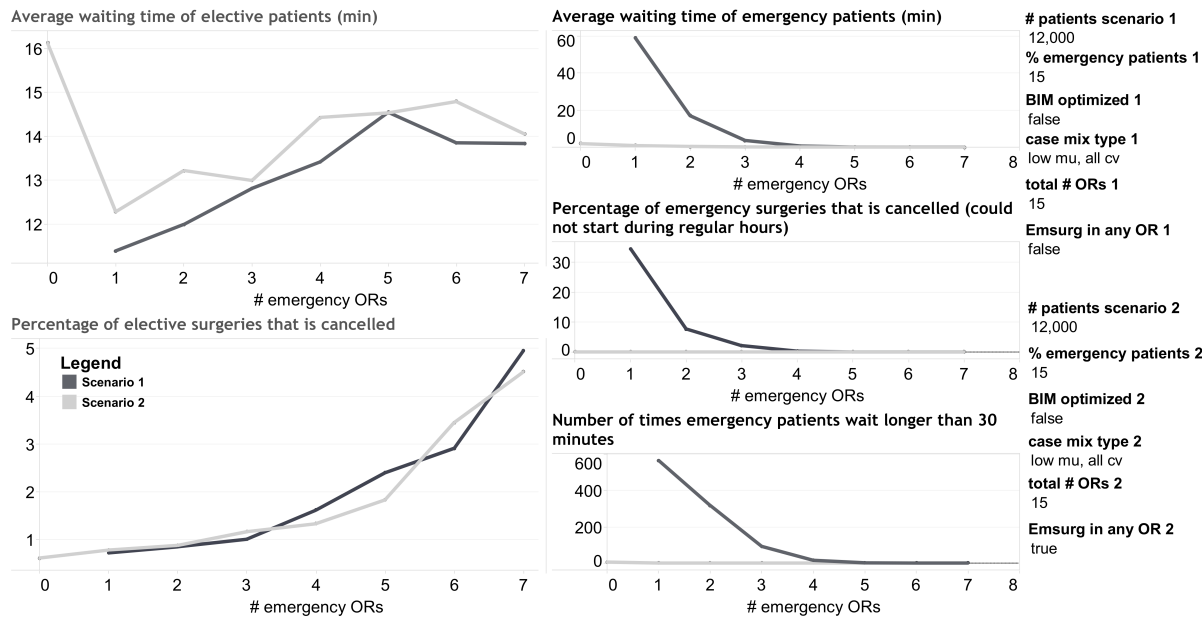


\subsubsection{Evaluating BIM effectiveness}

The use of break in optimization may further improve the effect of using a break in- or hybrid policy, as waiting times for emergency surgeries are minimized by reorganizing the elective program. Figure 5.15 shows the performance when using Policy 2 or 3, with and without the use of BIM. For completeness a similar setting with Policy 1 is displayed. In the evaluated setting there are 11000 patient arrivals, of which $20 \%$ are emergency patients. In total there are 10 ORs, and the patient case mix 4 is used.

Figure 5.15 BIM comparison

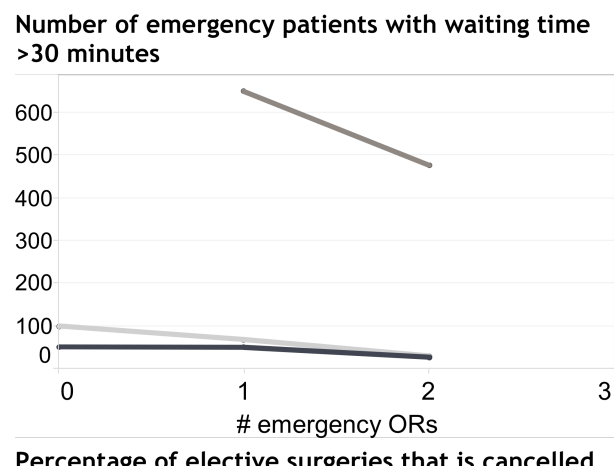

Average waiting time emergency patients $(\mathrm{min})$

80

60

40

20

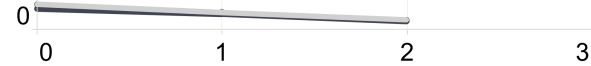

Percentage of elective surgeries that is cancelled Average overtime per ORday (min)

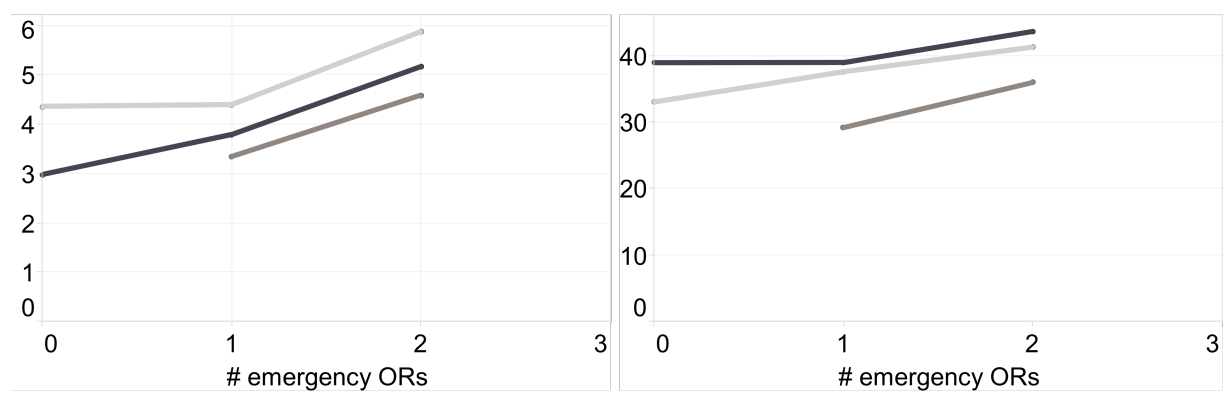

Legend
Flexible Policy (2 and 3), no BIM $\square$ Flexible Policy (2 and 3), BIM Dedicated Policy (1)

We find that, when using BIM, performance for emergency patients improves by both reducing the average waiting time and number of times emergencies wait over 30 minutes. Besides the improvements for emergency patients, the number of elective cancellations decreases. This can be explained by the fact that, by using BIM, the break in moments are not only spread more evenly throughout the day, they are also spread more evenly across ORs. This in turn more evenly distributes work across the ORs, and reduces cancellations.

Besides the illustrated case, we also evaluate the effect of using BIM under several other settings, specifically the number of emergency patients, operating theater size, case mix, and overall load. We find that in all cases the use of BIM improves emergency patient performance, and performs comparatively better in 


\section{Chapter 5. Emergency OR or not: A simulation study of emergency surgery scheduling policies}

scenarios where there are higher overall loads or surgery types with longer durations (e.g., case mix 3). Of the settings we examined, average waiting time for emergency patients decreased between 0.2 and 0.8 minutes. While the use of BIM improves Policy 2 and 3, and does not come at the expense of other performance indicators, the increases in performance are not large enough to change the point(s) of viability mentioned in Section 5.6.2. Even when using BIM, there needs to be a large enough number of total ORs for breaking in to be viable.

\subsection{Conclusions}

In this research we evaluated different policies that are used in practice to deal with emergency patients arriving at the operating theater. Three policies evaluated are: the use of dedicated ORs for emergency patients (Policy 1), letting emergency patients break into the elective program (in regular ORs, Policy 2), and a combination of the two aforementioned policies (Policy 3). We evaluated the different policies using a discrete event simulation model, and incorporated the results in a tool to enable quick comparison of alternative policies and settings. This evaluation is carried out for an extensive set of scenario settings such as operating theater size and patient case mix, as well as multiple stakeholder KPIs including utilization, elective and emergency waiting times, and overtime. In total, 320.000 different scenario settings were simulated.

Generally, hospitals aim to have both acceptable waiting times and an acceptable utilization. It is however hospital dependent what is defined as acceptable. This is also reflected in the reviewed literature, as papers use different definitions of performance indicators, or different indicators all together. These differences make a direct comparison difficult, and it may lead to conflicting conclusions. In general, we find there is a trade-off in performance indicators between using a dedicated (1) or break-in (2) policy. Using dedicated emergency ORs leads to lower waiting times for elective patients, as there are no break-ins, but outliers in waiting times for emergency patients are more likely. Once all emergency ORs are in use, arriving emergency patients may have to wait considerably longer than under a break-in policy. Also, it seems that dedicated policies are less robust under the higher utilized systems, as the number of elective cancellations is almost always higher than under its break-in counterpart, indicating that more rescheduling would be required. This makes sense, as under a break-in policy, capacity may be better utilized (e.g., there are no possibly empty emergency ORs), leading to better utilization during regular hours and less cancellations.

The main characteristic influencing the effectiveness of the policies is the operating theater size. We find that for smaller operating theaters it is more beneficial to use a dedicated policy, as there are not enough ORs to allow for a timely break in into the elective program. In our evaluated settings, we find the minimum required number of ORs lies around 8 to 9 ORs (in total) before break-in policies (2-3) become viable. With a lower number of ORs, a dedicated Policy (1) outperforms a flexible approach, and with more ORs the trade-off in 
performance becomes visible.

Besides the trade-off in performance between Policy 1 and Policy 2 we also evaluated a hybrid policy (3). In our compared setting, the hybrid policy outperformed the other policies as an intermediary solution that is better tuned to the specific underlying hospital case. Under Policy 3 there are some emergency ORs that ensure there are not too many break-ins in the elective program, keeping both elective and emergency waiting times low. However, if there are many emergency arrivals the elective ORs may be used for emergencies, ensuring emergency patients are treated in a timely manner, and keeping outliers in waiting times low.

In addition to the policies we also evaluated the use of BIM optimization as an approach to further optimize the break-in and hybrid policies. Using BIM is found to decrease average emergency waiting times, as well as number of emergencies waiting over 30 minutes regardless of scenario settings. However, its effects seem more beneficial when there is more variability in the patient case mix, and the overall system load is high. Besides the improvements for emergency patients, performance for elective patients remains similar. In practice, while some ordering of patients is dictated for medical reasons, using BIM can thus result in increased performance without additional costs, as it can be implemented without organizational changes.

We did not take into account the costs associated with a break-in or hybrid policy. In practice, if emergency patients are to be treated in regular ORs additional equipment may be needed to facilitate this, which also introduces the financial differences between policies. Similarly, we assume change over times are incorporated in the surgery times, which may increase when treating emergency patients in regular ORs as additional steps must be taken before starting treatment. For future work, it may be interesting to take such additional effects into account.

The use of BIM shows that the scheduling of patients during the day influences OR performance. As such, other more dynamic allocation policies of patients into the ORs may be interesting to investigate in future work. For example, inserting breaks into the elective program may further reduce emergency patient waiting times when using Policy 2 or 3. Alternatively, taking into account expected surgery completion times before allocating emergency patients to an elective OR may further complement break-in policies. By only allocating emergency patients to elective ORs when emergency ORs are not expected to be available soon may reduce emergency waiting times outliers while reducing elective program disruptions.

Besides comparing our simulation results, we also placed some previous studies within our framework and found similar results, validating our outcomes. However, in general we found it difficult to compare studies, as settings often differ greatly, and are often not completely defined. A more generalized approach to standardize reporting on operating room planning literature could be developed, incorporating often omitted settings such as arrival process details and available 
capacity per OR.

In this study, we explore the relationship between policies, and hospital characteristics such as case mix, hospital size, and number of emergency patients for all possible cases. Our generalized approach and extensive simulations allow for the comparison of policies not only for a specific hospital case, but also across cases. We evaluated all performance indicators prominently mentioned in the literature to allow for a full comparison between settings. We aggregated all simulation results in the OR analyzer (available at: http://tabsoft.co/29jrDwM) to allow healthcare practitioners to evaluate policies and performance for their specific case setting, as well perform what-if analyses. Furthermore, we discuss the effect of the policies under different hospital sizes, case mixes, number of elective and emergency patients, and use of BIM optimization. The discussed results however by no means encompass all insights to be gained from the OR analyzer tool, and only show some of the comparisons that can be made using the tool. We encourage readers to further explore and use the tool as they see fit. 


\section{Surgical procedure type scheduling incorporating semi urgent patients}

\subsection{Introduction}

In operating room (OR) scheduling, a tactical (cyclic) block schedule in which OR blocks are assigned to surgical specialties is often used. Once surgical demand is realized, surgical cases are scheduled into the allocated OR blocks. In practice, this often results in each planning period having many of the same type of surgical cases being scheduled, with some slight variations due to changes in surgical demand. Additionally, changes and cancellations often take place as processes within the OR are stochastic in nature. Some of this stochasticity may be accounted for when scheduling surgical cases. For example, to cope with uncertain surgery durations, slack may be incorporated within a schedule [196].

A source of uncertainty that is often not accounted for in the literature (as we shall argue in Section 6.2), is the arrival of semi-urgent patients. Emergency patients that must be treated on the day of arrival, are either treated in dedicated emergency ORs or accommodated by modifying the elective program. Semi-urgent patients, however, may be postponed for some limited time (e.g., several days or weeks). To accommodate these semi-urgent patients, surgical case schedules are often changed to ensure timely treatment. This research is motivated by a large Dutch hospital where part of the recurring surgery demand consists of semi-urgent patients who should be operated within several days. In order to reduce the rescheduling of patients after semi-urgent patient arrivals, an approach is sought that takes these patients into account on a tactical level.

In this research we aim to create a surgery type schedule (STS) that can hierarchically be positioned between the tactical level block schedule and operational level surgical case assignment [102]. We take into account both elective and semi-urgent surgery demand such that the waiting time of semi-urgent patients is minimized, as well as the required OR capacity. In addition, by incorporating semi-urgent surgeries on the tactical level, fewer changes and cancellations take place during implementation of the surgery schedule. Our contribution is that we design a solution approach to create surgery type schedules that specifically incorporate semi-urgent surgery demand. We account for stochastic surgery durations 


\section{Chapter 6. Surgical procedure type scheduling incorporating semi urgent patients}

and stochastic semi-urgent surgery arrivals. Finally, we apply and evaluate our approach using a hospital case study and demonstrate that good approximate surgery type schedules may be constructed within a reasonable time-frame.

This chapter is organized as follows: Section 6.2 discusses relevant literature on operating room planning and scheduling. Section 6.3 presents the mathematical problem description and solution methods, and Section 6.4 presents our results. Finally, Section 6.5 contains conclusions and future research directions.

\subsection{Literature}

There is a considerable amount of literature on operating room planning and scheduling, with multiple literature reviews carried out in recent years $[30,40$, $70,89,114,238]$. Our interest is in surgery scheduling approaches that account for semi-urgent or emergency demand when creating OR schedules.

Non-elective patients have received little attention compared to elective patients in OR planning and scheduling, while the arrival of non-electives is one of the major causes of operational deficiencies [40]. Emergency patients are often taken into account by reserving some available capacity for all emergency patients that arrive such that overtime or cancellations are acceptable. This is different from our problem, as we want to account for semi-urgent patients that may wait for several days, which gives us more options to plan these patients together with the elective patient program. Mathematical programming is often used to create OR schedules incorporating semi-urgent or emergency demand. [2] review varying ranges of slack and bin-interchange flexibility in allocating block time to patient groups to determine an optimal strategy in dealing with uncertain demand. Slack is additionally reserved OR capacity to deal with uncertainty in surgical duration. These strategies were derived from earlier research [54] but expanded to allow uncertainty. The paper shows a clear trade-off between utilization, service level and overtime by adjusting the amount of slack. [241] provide a similar insight through a simulation study of four extreme operating room planning strategies that focus on different objectives, such as maximize resource utility, minimize access times and maximize staff preferences. Another simulation study examines the trade-off between utilization and service level in reserving capacity for emergency patients [32]. A limitation of these studies is that only one surgery type is taken into account $[32,241]$. Other studies similarly aim to schedule elective surgeries, and account for add-on emergencies that must be treated on the day of arrival [203, 262].

[263] present a queuing theory model that is used to determine expected semiurgent patient access times with a given arrival rate and capacity. Following this they determine a policy whether to postpone semi-urgent patients to next week, or cancel elective patients, using a Markov Decision Process. [230] present a mathematical programming model to schedule patients based on medical priority instead of surgical duration. Unfortunately, the model has some limitations, such as no uncertainty in arrival rate or surgical duration, no emergency patients and 
excess demand. Another approach to schedule patients based on medical priority uses dynamic programming [186]. In this model, a trade-off between surgery overtime and postponement costs result in a surgical schedule. All surgery types and durations however are assumed to be identically distributed, and a relaxation of this assumption increases the model complexity dramatically.

Various authors use Sample Average Approximation (SAA) to model uncertain emergency demand and surgery time requirements. Herein the objective function of a stochastic problem is approximated using a set of generated scenarios. [155, 156] use SAA to model the operating room planning problem in this manner, however they assume fixed surgery times. They find that by incorporating the stochastic aspect of emergency arrivals, costs may be significantly reduced. [185] use SAA to plan elective surgeries while accounting for uncertain surgery durations and downstream bed availability. [247] allocate elective surgical cases, accounting for unscheduled emergency arrivals by incorporating slack. Using SAA they transform their stochastic model that incorporates uncertain emergency demand into a deterministic integer linear program (ILP). Hereafter, they use column generation to allocate surgeries such that cancellations of elective patients are minimized.

Another scenario based approach by [207] similarly includes uncertain surgery durations and required time for emergencies. [77] use a scenario based modeling approach wherein they approximate the likelihood of a scenario using a discretization of (elective) surgery time distributions. With this approach, they assign, sequence and determine starting times for elective patients, while accounting for emergency arrivals that must be treated on the day of arrival.

Concluding, the literature incorporating emergency demand often only models emergency demand that must be treated on the day of arrival. However, in our problem setting, semi-urgent patients must be seen within several days. By accounting for the stochastic semi-urgent surgery arrivals in a surgery type scheduling approach, a better OR planning may be created than when accounting for uncertain arrivals as a single (additional) claim on OR capacity. In addition, this specific incorporation of semi-urgent surgeries ensures fewer last-minute cancellations and changes to the OR schedule.

To our knowledge, the scheduling of semi-urgent patients in this manner has not been addressed in the literature. We schedule semi-urgent patients by building upon previous work on OR scheduling, and herein we incorporate the stochasticity of semi-urgent patient arrivals, as well as surgery durations. We use an ILP approach and incorporate uncertain patient arrivals via SAA, and surgery durations by incorporating slack time in our OR schedules to reduce overtime.

\subsection{Model formulation and solution approach}

In this section, we first give a formal problem description in Section 6.3.1. Section 6.3.2 outlines our solution approach, and Section 6.3.3 details our heuristic to construct a feasible STS. 


\section{Chapter 6. Surgical procedure type scheduling incorporating semi urgent patients}

\subsubsection{Explicit model formulation}

The set $T$ denotes the days in the planning horizon, and $J$ denotes the identical ORs. The set $I$ denotes the surgery types to be scheduled, wherein we specify $I_{e}\left(I_{e} \subset I\right)$ and $I_{u}\left(I_{e} \subset I\right)$ as the elective and semi-urgent surgery types respectively. In addition, we consider the sets of subspecialties $S$ and OR sizes $R$. The operating room sizes represent the different sizes a created schedule may have (e.g., half days and full days). Finally, the set $\Xi$ contains the realizations of semi-urgent patient arrivals that can occur during the planning horizon. Each scenario $\zeta \in \Xi$ defines the realization of newly arriving semi-urgent patients during the planning horizon. Let $\phi(\zeta)$ be the probability of scenario $\zeta \in \Xi$, and $\sum_{\zeta \in \Xi} \phi(\zeta)=1$. We denote $\lambda_{i, t}^{\zeta}$ as the number of semi-urgent patients of type $i$ $\left(i \subset I_{u}\right)$ arriving on day $t \in T$ under scenario $\zeta \in \Xi$ (note that these patients can be operated from day $t$ onwards).

The decision variable $z_{i, j, r, t}$ characterizes the number of surgeries of type $i \in I$ to combine in a schedule of size $r \in R$ in OR $j \in J$ and day $t \in T$. In addition, the (binary) variable $w_{j, r, t, s}$ denotes if a surgeon of subspecialty $s \in S$ is assigned to OR $j \in J$, on day $t \in T$ with a schedule of size $r \in R$. We also formulate the (binary) variable $v_{j, r, t}$ which denotes if OR $j \in J$ on day $t \in T$ with schedule of size $r \in R$ is in use (e.g., there are surgeries assigned to it).

As a result of the assignment of surgery types to days in the planning horizon and arrivals of semi-urgent patients, there may be (semi-urgent) patients waiting for treatment. We model the waiting times based on an ILP formulation that allocates resources and plans elective patients on a tactical level [115]. Herein multiple care processes are modeled, and the number of patients to treat at particular stages of their care pathway is determined taking into account the waiting time of patients at each care process. We introduce the waiting list $N W_{i, t}^{\zeta}=\left(N W_{i, t, 0}^{\zeta}, N W_{i, t, 1}^{\zeta}, \ldots\right)$ where $N W_{i, t, k}^{\zeta}$ denotes the number of patients of type $i \in I_{u}$ waiting for $k$ days at the start of day $t \in T$ in realized scenario $\zeta \in \Xi$. Similarly, we formulate $N T_{i, t, k}^{\zeta}$ as the number of treated patients of type $i \in I_{u}$, that have waited for $k$ days on day $t \in T$ under realized scenario $\zeta \in \Xi$. We aim to minimize the weighted waiting time of semi-urgent patients, and also minimize the required OR time to meet surgery demand. Table 6.1 gives the sets, indices, variables and parameters used within our problem formulation. 
Table 6.1 Notation introduced in Section 6.3.1

\begin{tabular}{|c|c|}
\hline Notation & description \\
\hline \multicolumn{2}{|l|}{ Sets/indices: } \\
\hline$T ;(t \in T)$ & Days \\
\hline$K ;(k \in K)$ & Days spent waiting \\
\hline$S ;(s \in S)$ & Specialty \\
\hline$J ;(j \in J)$ & Operating rooms \\
\hline$R ;(r \in R)$ & Operating room sizes \\
\hline$\Xi ;(\zeta \in \Xi)$ & Scenarios \\
\hline$I ;(i \in I)$ & Patient (procedure) types \\
\hline$I_{e} ;\left(I_{e} \subset I\right)$ & Elective patient (procedure) types \\
\hline$I_{u} ;\left(I_{u} \subset I\right)$ & Urgent patient (procedure) types \\
\hline \multicolumn{2}{|l|}{ Variables: } \\
\hline $\mathrm{Z}_{i, j, r, t}$ & $\begin{array}{l}\text { Number of surgeries of type } i \text {, carried out in OR } j \\
\text { with size } r \text { on day } t \text {. }\end{array}$ \\
\hline $\mathrm{W}_{j, r, t, s}$ & $\begin{array}{l}\text { Binary variable indicating if a surgeon of specialty } s \\
\text { is assigned to OR } j \text { with size } r \text { on day } t \text {. }\end{array}$ \\
\hline $\mathrm{V}_{j, r, t}$ & $\begin{array}{l}\text { Binary variable indicating if OR } j \text { with size } r \text { is opened } \\
\text { on day } t \text {. }\end{array}$ \\
\hline $\mathrm{NW}_{i, t, k}^{\zeta}$ (NumWait) & $\begin{array}{l}\text { Number of surgeries of type } i \text { waiting at the start of } \\
\text { day } t \text {, that have waited } k \text { days in scenario } \zeta \text {. }\end{array}$ \\
\hline $\mathrm{NT}_{i, t, k}^{\zeta}($ NumTreat $)$ & $\begin{array}{l}\text { Number of surgeries of type } i \text { to plan on day } t \text {, that } \\
\text { have waited } k \text { days in scenario } \zeta \text {. }\end{array}$ \\
\hline $\mathrm{NTD}_{i, t}^{\zeta}$ (NumTreatDay) & $\begin{array}{l}\text { Number of surgeries of type } i \text { to plan on day } t \text { (over } \\
\text { all } k \text { ) in scenario } \zeta \text {. }\end{array}$ \\
\hline \multicolumn{2}{|l|}{ Parameters: } \\
\hline$\sigma_{w}$ & Weight of waiting time objective. \\
\hline$\sigma_{u}$ & Weight of OR utilization objective. \\
\hline$\beta_{i, k}$ & $\begin{array}{l}\text { Weight of patients of type } i \text {, that have waited for } k \\
\text { days. }\end{array}$ \\
\hline$\phi(\zeta)$ & Probability of scenario $\zeta$ occurring. \\
\hline$\lambda_{i, t}^{\dot{\zeta}}$ & $\begin{array}{l}\text { Number of arrivals of surgery type } i \text { on day } t \text { under } \\
\text { scenario } \zeta \text {. }\end{array}$ \\
\hline$a_{j, t}$ & binary indicating the availability of $\mathrm{OR} j$ on day $t$. \\
\hline$b_{i, s}$ & $\begin{array}{l}\text { Binary indicating if surgery type } i \text { is carried out by } \\
\text { specialty } s \text {. }\end{array}$ \\
\hline$c_{t, s}$ & Number of available surgeons of specialty $s$ on day $t$. \\
\hline$d_{r}$ & Capacity of an OR of size $r$. \\
\hline$e_{r}$ & Cost of using an OR of size $r$. \\
\hline$s_{i}$ & Demand of surgery type $i$. \\
\hline$m_{i}$ & $\begin{array}{l}\text { Maximum number of surgeries of type } i \text { that can be } \\
\text { carried out in an OR of largest size } r \text {. }\end{array}$ \\
\hline$\alpha$ & Maximum accepted probability of overtime of an OR. \\
\hline$\hat{k}$ & $\begin{array}{l}\text { Maximum recorded waiting time of urgent patients (in } \\
\text { days). }\end{array}$ \\
\hline
\end{tabular}




\section{Chapter 6. Surgical procedure type scheduling incorporating semi urgent patients}

With the notation from Table 6.1 we formulate the mathematical model for our problem as follows:

$$
\begin{aligned}
& \min \sum_{r} \sum_{t} \sum_{j}\left(V_{j, r, t} e_{r}\right) \sigma_{u}+\sum_{\Xi} \sum_{i} \sum_{t} \sum_{k=e_{i}}\left(\phi(\zeta) N W_{i, t, k}^{\zeta} \cdot \beta_{i, k}\right) \sigma_{w} \\
& Z_{i, j, r, t} \leq V_{j, r, t} m_{i} \quad \forall i \in I, j \in J, r \in R, t \in T \\
& \sum_{j} \sum_{r} \sum_{t} Z_{i, j, r, t} \geq s_{i} \quad \forall i \in I_{e} \\
& \sum_{r} V_{j, r, t} \leq a_{j, t} \quad \forall j \in J, t \in T \\
& \sum_{j} \sum_{r} W_{j, r, t, s} \leq c_{t, s} \quad \forall t \in T, s \in S \\
& Z_{i, j, r, t} \leq \sum_{s} b_{i, s} W_{j, r, t, s} m_{i} \\
& \forall i \in I, j \in J, r \in R, t \in T \\
& \sum_{s} W_{r, t, j, s} \leq 1 \quad \forall j \in J, r \in R, t \in T \\
& N W_{i, t, k}^{\zeta}=\lambda_{i, t}^{\zeta} \quad \forall i \in I_{u}, t \in T, \zeta \in \Xi, k=0 \\
& N W_{i, t, k}^{\zeta}=N W_{i, t-1, k-1}^{\zeta}-N T_{i, t-1, k-1}^{\zeta} \\
& \forall i \in I_{u}, t>0, \zeta \in \Xi, k>0 \\
& N W_{i, t, k}^{\zeta}=N W_{i, t-1, k-1}^{\zeta}-N T_{i, t-1, k-1}^{\zeta} \\
& +N W_{i, t-1, k}^{\zeta}-N T_{i, t-1, k}^{\zeta} \\
& \forall i \in I_{u}, t \in T, \zeta \in \Xi, k=\hat{k} \\
& N T D_{i, t}^{\zeta}=\sum_{k} N T_{i, t, k}^{\zeta} \quad \forall i \in I_{u}, t \in T, \zeta \in \Xi \\
& N T D_{i, t}^{\zeta} \leq \sum_{r, j} Z_{i, j, r, t} \quad \forall i \in I_{u}, t \in T, \zeta \in \Xi \\
& P\left(f_{j, r, t}(\hat{z}) \leq d_{r}\right) \geq 1-\alpha \quad \forall j \in J, r \in R, t \in T \\
& V_{j, r, t} \in\{0,1\} \quad \forall j \in J, r \in R, t \in T \\
& W_{j, r, t, s} \in\{0,1\} \quad \forall j \in J, r \in R, t \in T, s \in S \\
& Z_{i, j, r, t} \in \mathbb{N} \quad \forall i \in I, j \in J, r \in R, t \in T
\end{aligned}
$$

The objective of the model is to minimize time spent waiting for urgent patients past their due date (in days), and the total required number of ORs to treat elective and urgent patients. Constraint (6.2) stipulates that ORs must be opened if patients are to be scheduled in it, and constraint (6.3) ensures that enough elective patients are scheduled to meet demand requirements. Constraints (6.4) and (6.5) require that ORs and specialists of the proper specialty 
are available when scheduling surgeries, and constraints (6.6) and (6.7) ensure that only one specialty is assigned to an OR, and all surgeries within an OR belong to that same specialty. Constraints (6.8)-(6.11) ensure that the waiting list variables are consistent, and untreated semi-urgent patients per time period are updated. Constraint (6.8) formulates the new arrivals for each day under a scenario realization, and constraints (6.9) and (6.10) update the waiting lists at each time period, accounting for treated semi-urgent patients. For tractability, the number of time periods semi-urgent patients are waiting is bounded by some value $\hat{k}$. Constraint (6.10) updates the waiting list at the maximum (recorded) waiting time of patients. Constraints (6.11) and (6.12) link the number of treated semi-urgent patients per day with the assignment of surgeries to schedules. Finally, constraint (6.13) ensures that the overtime probability of an OR schedule does not exceed some given probability $\alpha$. To formulate this constraint we denote $f_{r, t, j}(\hat{z})$ as the probability distribution of the total session time of surgeries scheduled of schedule $r$ on day $t$ in OR $j$, where $\hat{z}$ is the vector of all variables $Z_{i, j, r, t}$. Since the number of possible scenarios is typically very large, the resulting model is too difficult to solve for realistic instance sizes. Also, in practice, solutions of (sufficient) quality obtained within reasonable time are preferred over an optimal solution obtained after several hours or even days. Therefore, to obtain a good solution within reasonable time, we propose a decomposition approach in the next section.

\subsubsection{Solution approach}

We use a decomposition approach wherein we first address the allocation of semiurgent surgery types, followed by the allocation of elective surgery types. We do this as follows:

- First, we allocate semi-urgent surgery types (i.e. when and how many surgery types to plan of each type for each OR-day), such that waiting time is minimized.

- Second, we take the allocation of semi-urgent surgery types as input, and combine these with elective demand to create a STS that minimizes the required number of ORs.

Specifically, we use a SAA method to minimize waiting time for semi-urgent surgery types, followed by a column generation (CG) based heuristic procedure to allocate elective and semi-urgent surgery types to STS. This is done by combining those elective and semi-urgent surgery types that best absorb the required slack to guarantee an acceptable overtime probability caused by uncertain surgery durations. In the following subsections we describe our used SAA method (6.3.2.1) and CG reformulation (6.3.2.2 and 6.3.2.3). 


\subsubsection{Semi-urgent patient scheduling using SAA}

To address the uncertainty of semi-urgent surgery demand inherent in our elective and semi-urgent surgery planning problem we use an SAA approach. This approach is based on previous work that applied SAA to incorporate uncertain emergency demand during the day [155, 156, 247]. In our problem however we apply it to the entire planning horizon and allocate available semi-urgent surgery capacity such that waiting times are minimized. Herein the objective function is approximated by using a random selection of the stochastic scenarios $[4,139]$. We generate $N$ random scenario samples $\left(\zeta^{1}, \ldots, \zeta^{N}\right)$ from the given (discrete) distributions underlying the arrival of semi-urgent patients, and approximate the value of $\sum_{\zeta} \sum_{i} \sum_{t}\left(\phi(\zeta) N W_{i, t, k}^{\zeta} \cdot \beta_{i, k}\right)$ by $\frac{1}{N} \sum_{n} \sum_{i} \sum_{t}\left(N W_{i, t, k}^{n} \cdot \beta_{i, k}\right)$. The following mathematical model then lists the SAA formulation of the stochastic semi-urgent surgery planning problem with a sample size of $N$. 


$$
\begin{aligned}
& \min \frac{1}{N} \sum_{n} \sum_{i} \sum_{t}\left(N W_{i, t, k}^{n} \cdot \beta_{i, k}\right) \\
& Z_{i, j, r, t} \leq V_{j, r, t} M_{i} \quad \forall i \in I_{u}, j \in J, r \in R, t \in T \\
& \sum_{r} V_{j, r, t} \leq a_{j, t} \quad \forall j \in J, t \in T \\
& \sum_{r, j} W_{j, r, t, s} \leq c_{t, s} \quad \forall t \in T, s \in S \\
& Z_{i, j, r, t} \leq \sum_{s} b_{i, s} W_{j, r, t, s} m_{i} \quad \forall i \in I_{u}, j \in J, r \in R, t \in T \\
& \sum_{s} W_{j, r, t, s} \leq 1 \quad \forall j \in J, r \in R, t \in T \\
& N W_{i, t, k}^{n}=\lambda_{i, t}^{n} \quad \forall i \in I_{u}, t \in T, n \in N, k=0 \\
& N W_{i, t, k}^{n}=N W_{i, t-1, k-1}^{n}-N T_{i, t-1, k-1}^{n} \\
& \forall i \in I_{u}, t>0, n \in N, k>0 \\
& N W_{i, t, k}^{n}=N W_{i, t-1, k-1}^{n}-N T_{i, t-1, k-1}^{n} \\
& +N W_{i, t-1, k}^{n}-N T_{i, t-1, k}^{n} \quad \forall i \in I_{u}, t \in T, n \in N, k=\hat{k} \\
& N T D_{i, t}^{n}=\sum_{k} N T_{i, t, k}^{n} \quad \forall i \in I_{u}, t \in T, n \in N \\
& N T D_{i, t}^{n} \leq \sum_{r, j} Z_{i, j, r, t} \quad \forall i \in I_{u}, t \in T, n \in N \\
& \sum_{j} \sum_{r} \sum_{t} Z_{i, j, r, t} \leq s_{i} \quad \forall i \in I_{u} \\
& V_{j, r, t} \in\{0,1\} \quad \forall j \in J, r \in R, t \in T \\
& W_{j, r, t, s} \in\{0,1\} \quad \forall j \in J, r \in R, t \in T, s \in S \\
& Z_{i, j, r, t} \in \mathbb{N} \quad \forall i \in I, j \in J, r \in R, t \in T
\end{aligned}
$$

Given that only a fraction of all patients are semi-urgent (i.e. there is enough capacity to schedule only semi-urgent patients), we drop the OR capacity constraint, and only require that an OR and corresponding subspecialty are available when scheduling surgeries. Without electives to schedule in tandem, a solver would schedule as many urgent slots as possible to minimize waiting time. Therefore we limit the number of allowed semi-urgent surgeries per type $i$ via constraint (6.25) based on historical data. The final product of the SAA model details the number of patients and dates to schedule semi-urgent surgeries. This can be taken into account as a demand constraint when scheduling urgent patients alongside elective patients, by fitting elective patients such that the total number of required ORs is minimized (and thus use of ORs is maximized). We use the following general SAA algorithm [4]: 


\section{Chapter 6. Surgical procedure type scheduling incorporating semi urgent patients}

1. For $m=1, \ldots, M$ repeat the following steps:

1.1. Generate $N$ sample scenarios.

1.2. Solve the SAA problem of constraints (6.14)-(6.24), and let $\hat{X}_{N}^{m}$ be the solution vector and $\hat{v}_{N}^{m}$ the optimal objective value.

1.3. Generate $N^{\prime}$ independent random samples. Estimate the true objective value and variance using a feasible solution $\bar{X}$ and evaluate $\hat{g}_{N^{\prime}}(\bar{X})$ and $S_{\hat{g}_{N^{\prime}}(\bar{X})}^{2}$ using the following equations:

$$
\begin{aligned}
& \hat{g}_{N^{\prime}}(\bar{X})=\frac{1}{N^{\prime}} \sum_{n}^{N^{\prime}} \sum_{i} \sum_{t}\left(N W_{i, t, k}^{n} \cdot \beta_{i, k}\right), \\
& S_{\hat{g}_{N^{\prime}}(\bar{X})}^{2}=\frac{1}{N^{\prime}\left(N^{\prime}-1\right)} \sum_{n}^{N^{\prime}}\left[\sum_{i} \sum_{t}\left(N W_{i, t, k}^{n} \cdot \beta_{i, k}\right)-\hat{g}_{N^{\prime}}(\bar{X})\right] .
\end{aligned}
$$

2. Calculate $\bar{v}_{N}^{M}$ and $S_{\bar{v}_{N}^{M}}^{2}$ :

$$
\begin{aligned}
& \bar{v}_{N}^{M}=\frac{1}{M} \sum_{m=1}^{M} \hat{v}_{N}^{m}, \\
& S_{\bar{v}_{N}^{M}}^{2}=\frac{1}{M(M-1)} \sum_{m=1}^{M}\left[\hat{v}_{N}^{m}-\bar{v}_{N}^{M}\right]^{2} .
\end{aligned}
$$

3. For each solution $\hat{X}_{N}^{m}, m=1, \ldots, M$, estimate the optimality gap by $\hat{g}_{N^{\prime}}\left(\hat{X}_{N}^{m}\right)-\bar{v}_{N}^{M}$, and estimated variance of $S_{\bar{v}_{N}^{M}}^{2}+S_{\hat{g}_{N^{\prime}}\left(\bar{X}_{N}^{m}\right)}^{2}$.

Using this algorithm we obtain a lower and upper bound for the true objective value. Note that in step 1.3 we require a feasible solution $\bar{X}$. We obtain this solution by solving the SAA model for a sample size of $N$. Finally, a solution to the problem can be chosen from the replication sample $M$. For a detailed description of the algorithm we refer to [4].

\subsubsection{Minimizing the required number of ORs using column gener- ation}

After running the stochastic semi-urgent surgery planning model, we have determined when and how many surgery types to schedule of each semi-urgent surgery type $i$. This is taken as input, similar to the demand requirements of elective surgery types. A difficulty of our approach is that the number of surgery type allocations that are feasible is exceptionally large, and that stating all variables is not possible.

In order to model our OR scheduling problem we use a CG based formulation. This approach has been applied to OR scheduling [66, 67, 247], and shown to be able to solve large instances of realistic sizes that are otherwise not solvable. We use a CG formulation similar to [196], as this approach only works with a subset 
of the variables, adding more if needed [170]. Instead of allocating surgery types to ORs we use the concept of Operating Room Day Schedules (ORDSs) [196]. An ORDS $u(u \in U)$ is a combination of surgical procedures of various types $i$ that are allowed to be carried out together (i.e., satisfy resource constraints). Using CG we start with a small set of $U$, and add new ORDSs to this set that may improve our objective function.

In addition to the already introduced notation in Table 6.1, we introduce the decision variable $X_{r, u, t}$ which denotes the number of ORDSs of size $r$ and procedure set $u$ that are carried out on day $t$. To connect surgeries to ORDSs and specialties we denote $p_{r, u, i}$ as the number of surgeries of type $i$ carried out in an ORDS of type $u$ and size $r$, and the binary indicator $b_{r, u, s}$ that denotes whether ORDS $u$ of size $r$ is used by subspecialty $s$. The number of available ORs on day $t$ is denoted by $a_{t}$, and $c_{t, s}$ denotes the number of specialists of subspecialty $s$ that are available on day $t$. Finally, we formulate $\hat{s}_{i, t}$ as the required semiurgent surgery demand of type $i$ to be scheduled on day $t$ as determined by the SAA model. We can then model the allocation of ORDSs (primal model) to the planning horizon as follows:

$$
\begin{aligned}
\min \sum_{r} \sum_{u} \sum_{t} X_{r, u, t} e_{r} & \\
\sum_{r} \sum_{u} \sum_{t} X_{r, u, t} p_{r, u, i} & \geq s_{i} \quad \forall i \in I_{e} \\
\sum_{r} \sum_{u} \sum_{t} X_{r, u, t} p_{r, u, i} & \geq \hat{s}_{i, t} \quad \forall i \in I_{u}, t \in T \\
\sum_{r} \sum_{u} X_{r, u, t} & \leq a_{t} \quad \forall t \in T \\
\sum_{r} \sum_{u} X_{r, u, t} b_{r, u, s} & \leq c_{t, s} \quad \forall t \in T, s \in S \\
X_{r, u, t} & \leq \sum_{s} b_{r, u, s} a_{t} \quad \forall r \in R, u \in U, t \in T \\
X_{r, u, t} & \in \mathbb{N} \quad \forall r \in R, u \in U, t \in T
\end{aligned}
$$

The objective function (6.26) minimizes the number of required ORs, and constraints (6.27) and (6.28) ensure that sufficient elective and semi-urgent surgeries are planned. Constraints (6.29) and (6.30) require that schedules are only planned if ORs and surgeons of the related subspecialties are available. Finally, constraint (6.31) requires that an OR schedule $u$ is scheduled in a proper size $r$. In this reformulation each column corresponds to a feasible OR schedule $(r, u)$ that satisfies capacity and allocation constraints. By iteratively adding new OR schedules (columns) we can optimally solve the linear relaxation of the surgery planning problem, and then use a heuristic procedure based on the CG solution obtained to construct a feasible solution with satisfactory performance. In the next section we detail the pricing model that is used to construct new OR 


\section{Chapter 6. Surgical procedure type scheduling incorporating semi urgent patients}

schedules to add to the primal model.

\subsubsection{Pricing model}

Our primal model assumes that we know the ORDS set $U$ that make up the optimal schedule. However, as mentioned we use column generation to iteratively add ORDSs $u$ to a subset of $U$. This is done by solving the LP relaxation of the master problem. This master problem is the original primal model of Section 6.3.2.2 (with a subset of $U$ ). Solving the master problem results in shadow prices that we may use as input for the pricing problem. In this pricing problem we generate a new ORDS $u$ that is currently not included in the subset of $U$, but that may improve the master problem objective function. This way, we keep solving the (relaxed) master problem with an expanding subset of $U$, until no ORDS $u$ can be found in the pricing problems to increase the master problem objective function. The relaxed master problem solution with the created subset of $U$ is then optimal to the relaxed master problem which assumes the full set $U$ is known. We then use the created subset of $U$ to solve the original (unrelaxed) master problem, and obtain a feasible solution. In addition, we incorporate the randomness of surgery time durations in the pricing model by taking into account slack time such that the probability of overtime is limited.

Let $\pi_{i}, \delta_{i, t}, \gamma_{t}, \tau_{t, s}$, be the shadow prices of constraints (6.27)-(6.30) respectively. Let $Y_{i}$ be the decision variable denoting the number of surgeries of type $i$ carried out in the new ORDS. The pricing model creates a new ORDS $u$ for every size $r$, day $t$ and specialty $s$ that aims to violate the reduced cost criterion as much as possible. The reduced cost criterion is denoted by:

$$
\left(\sum_{i} Y_{i} *\left(\pi_{i}+\delta_{i, t}\right)\right)-\gamma_{t}-\delta_{t, s}
$$

Using the above reduced cost criterion as the objective function of our pricing problem, we try to create for each schedule size $r$, subspecialty $s$, and day of the planning horizon $t$ a new schedule $u$ that has a negative reduced cost. If such a schedule is found it is added to the set $U$ and the (relaxed) primal model is again solved to update the shadow prices.

In our pricing model we also have to take into account the overtime restrictions on an ORDS $u$, such that overtime occurs at most with probability $\alpha$. We assume that the underlying surgery times follow a normal distribution. The total duration of an ORDS is determined by the expected surgery time durations, the slack, which is determined by the variance of the surgeries carried out, and the safety factor $\alpha$. In order to model the overtime constraint (6.13) we use piecewise linear approximation [27], and determine the breakpoints as put forward by Bosch [31]. We assume that the total duration of an ORDS $u$ is normally distributed. Let $\mu_{u}$ and $\sigma_{u}^{2}$ be the mean and variance of the random variable $f_{u}(\hat{z})$ (i.e. $\left.f_{u}(\hat{z}) \sim \mathcal{N}\left(\mu_{u}, \sigma_{u}^{2}\right)\right)$. Taking $\mu_{i}$ and $\sigma^{2}$ as the mean and variance of the normally 
distributed duration of surgery type $i$, the overtime constraint of ORDS $u$ then becomes the sum over all surgeries carried out within it:

$$
\sum_{i} Y_{i} \mu_{i}+\phi^{-1}(1-\alpha) \sqrt{\sum_{i} Y_{i} \sigma_{i}^{2}} \leq d_{r}
$$

To piecewise approximate the surgery duration of normal distributed surgeries we introduce the set of $O(o=1, \ldots, O)$ breakpoints that separate each linear interval. Additionally, we introduce the breakpoint value $x_{o}$, the breakpoint function value $y_{o}$ and a weight for each breakpoint $\rho_{o}$. Each interval is described by a weighted sum of the breakpoints, which equals 1 [27]. For a full description on the determination on breakpoints we refer to [31]. Constraint (6.13) can then be replaced by the following constraints:

$$
\begin{aligned}
& \sum_{i} Y_{i} \mu_{i}+\phi^{-1}(1-\alpha) \sum_{o} \rho_{o} y_{o} \leq d_{r} \\
& \sum_{o} \rho_{o} x_{o}=\sum_{i} Y_{i} \sigma_{i}^{2} \\
& \sum_{o} \rho_{o}=1
\end{aligned}
$$

With the above piecewise linearization of overtime we are able to create a new OR schedule for every schedule size $r$, subspecialty $s$ and day in the planning horizon $t$ combination, as for each a different ORDS $u$ may improve the objective function. This gives us the following pricing model:

$$
\begin{gathered}
\max \sum_{i} Y_{i} *\left(\pi_{i}+\delta_{i, t}\right) \\
Y_{i} \leq b_{r, u, s} m_{i} \\
Y_{i} \leq \hat{s}_{i}, t \quad \forall i \in I_{u} \\
\sum_{i} Y_{i} \mu_{i}+\phi^{-1}(1-\alpha) \sum_{o} \rho_{o} y_{o} \leq d_{r} \\
\sum_{o} \rho_{o} x_{o}=\sum_{i} Y_{i} \sigma_{i}^{2} \\
\sum_{o} \rho_{o}=1 \\
Y_{i} \in \mathbb{N} \quad \forall i \in I
\end{gathered}
$$

The model's objective function (6.37) aims to find the combination of surgeries that has the largest reduced cost (see (6.32)), and if the reduced cost is negative the new ORDS is added to the set $U$. Constraint (6.38) ensures that only surgeries 


\section{Chapter 6. Surgical procedure type scheduling incorporating semi urgent patients}

of the available (assigned) specialty $s$ are scheduled, and constraint (6.39) limits the number of added semi-urgent surgeries to the new ORDS to the required daily demand. This constraint ensures that no ORDS are created with too many semi-urgent slots. Finally, constraints (6.40)-(6.42) limit the overtime probability of the new ORDS based on the surgeries carried out, and on the slack factor $\alpha$. In the next section we detail the heuristic approach used to find feasible OR schedules using the CG formulation.

\subsubsection{Heuristic approach}

We combine our solution approach from Section 6.3.2 with a heuristic in order to obtain a feasible STS. To initialize the CG procedure we require a set of ORDSs $U$ that allow us to obtain a feasible solution of the planning problem. We construct these initial schedules as follows:

For each surgery type $i$ and schedule size $r$ we create an ORDS by adding as many surgeries of that surgery type to the schedule, limited to the required daily $\left(\hat{s}_{i, t}\right)$ or monthly $\left(s_{i}\right)$ demand (for respectively semi-urgent and elective surgery types) and schedule size $r$ capacity.

1. If surgery type $i$ is semi-urgent repeat the following for every unique daily demand $\hat{s}_{i, t}$. If $i$ is an elective surgery type only do the following once using monthly demand $s_{i}$ :

1.1. Schedule at most the required demand $\left(\hat{s}_{i, t}\right.$ or $\left.s_{i}\right)$, or however many surgery types $i$ fit into the ORDS of size $r$ :

$$
Y_{i}=\min \left\{\frac{s_{i}}{\hat{s}_{i, t}}, \underset{i}{\arg \max }\left\{\sum_{i} Y_{i} \mu_{i}+\phi^{-1}(1-\alpha) \sqrt{\sum_{i} Y_{i} \sigma_{i}^{2}} \leq d_{r}\right\}\right\} .
$$

1.2. Sort the remaining surgery types in $I$ from longest to shortest (expected) surgery time.

1.3. While there are surgery types $i^{\prime} \in I$ remaining do the following:

i. If surgery type $i^{\prime}$ fits in the ORDS add the surgery type to the ORDS.

ii. Remove $i^{\prime}$ from the list of sorted surgery types.

By creating the ORDS in this manner we enable that an ORDS is created for each surgery type $i$, and that the ORDS contains many surgeries, properly utilizing the available capacity. In addition, this heuristic ensures that no schedules are created that would be useable when solving the relaxed master problem, without being an actual schedule candidate. For example, an ORDS with a number of semi-urgent surgery types that exceeds the daily requirement $\hat{s}_{i, t}$, would always be usable (fractionally) by the solver. In turn, this would limit the ability to find new schedules, because with the schedule with overcapacity a negative reduced cost may not be obtained.

As mentioned, when solving our problem using the CG formulation it is likely that a fractional solution is obtained. Therefore we use a heuristic procedure to 
find a feasible solution by iteratively including one ORDS $\left(X_{r, u, t}\right)$ in the final solution, based on the fractional (relaxed) master problem solution. We select the ORDS as follows:

1. Select the ORDS $X_{r, u, t}$ that treats the most semi-urgent patients:

$$
\arg \max _{u}\left(\sum_{i}^{I_{u}} X_{r, u, t} \cdot p_{r, u, i}\right) .
$$

2. If several decision variables $X_{r, u, t}$ treat an equal amount of semi-urgent patients, select the largest of those decision variables:

$$
\arg \max _{u} X_{r, u, t}
$$

3. If there are several integer or same sized fractional variables $X_{r, u, t}$, select the decision variable with the lowest cost:

$$
\arg \min _{u}\left(e_{r} \mid X_{r, u, t}\right)
$$

4. Finally, if there are multiple decision variables remaining with equal cost, randomly select one of those decision variables.

Directly prioritizing the most used ORDS, may enable to find better final solutions, as it is then more likely that highly used or even integer ORD assignments are selected. Ignoring semi-urgent assignments however, often resulted in early termination as ORDS were fixed such that semi-urgent demand could not be met. Using this prioritization when selecting an ORDS we found that our heuristic approach was often able to find good solutions without terminating early.

After selecting a decision variable $X_{r, u, t}$ we remove the surgery type demand planned in the fixed ORDS, and update the OR and surgeon availability. We then set this reduced problem as our new master problem, and again solve it using CG. This is repeated until an integer solution is found, or until no more surgery types need to be scheduled. Figure 6.1 shows our approach. Note that it may be possible that an ORDS is fixed, resulting in infeasibility as there are no more specialists or ORs available but there is demand remaining. In this case, the created set of ORDSs $U$ may be used to solve the master problem and obtain a feasible solution.

\subsection{Results}

In this section we detail the results of our SAA model (6.3.2.1) and CG formulated ORDS creation and planning model (6.3.2.2) using a heuristic approach. We first evaluate our approach using test instances, followed by application to our case study. Section 6.4.1 describes the test instances and input parameters. In Section 6.4.2 we present numerical results for the CG model. Following our numerical experiments, Section 6.4.3 presents our case study and in Section 6.4.4 
Figure 6.1 Approach framework including SAA and CG based heuristic

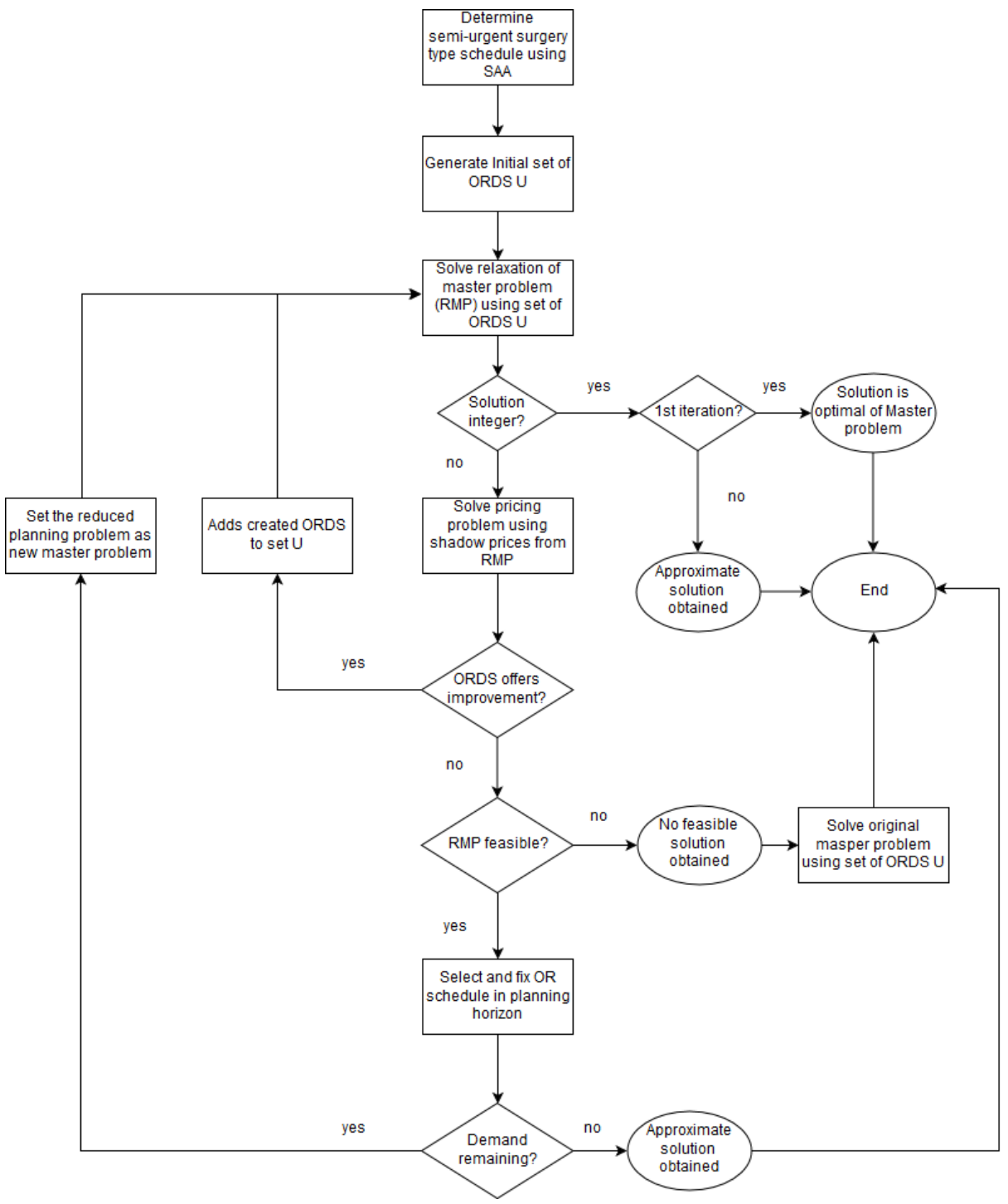

we determine the input parameters for the SAA model based on our case study, and present the case study results. The models were formulated using the AIMMS modeling software, and experiments were run on an Intel i7 $2.9 \mathrm{GHz} \mathrm{PC}$ with $8 \mathrm{~GB}$ of RAM. 


\subsubsection{Input parameters}

Here we describe the test instances and input parameters summarized in Table 6.2. In our test instance we consider a setting with varying subspecialties $(|S|=$ $1,3,6,9)$, and planning horizons $(|T|=14,28)$ of two and four weeks respectively. As OR sizes we take full and half OR days of eight and four hours respectively $(R=\{240,480\})$. We vary the number of different surgery types to schedule $(|I|=30,40,50,60)$, of which six surgery types are semi-urgent $\left(\left|I_{u}\right|=6\right)$.Also, we vary the sum of surgery type demand $\left(\sum_{i} s_{i}=50,100,200,300\right.$ and $\sum_{i, t} \hat{s}_{i, t}=$ $10,25,50,75)$. For each surgery type in $I$, the normal distribution parameters

Table 6.2 Experiment settings

\begin{tabular}{lc}
\hline Set/Parameter & Value \\
\hline$|T|$ & 14,28 \\
$|S|$ & $1,3,6,9,12$ \\
$\left|I_{e}\right|$ & $24,34,44,54$ \\
$\left|I_{u}\right|$ & 6 \\
$|J|$ & 6 \\
$|R|$ & 240,480 \\
$e_{r}$ & $0.5,1$ \\
$\alpha$ & $30 \%$ \\
$\hat{k}$ & 8 \\
$\sum_{i} s_{i}$ & $50,100,200,300$ \\
$\sum_{i} \sum_{t} \hat{s}_{i, t}$ & $10,25,50,75$ \\
\hline
\end{tabular}

are randomly generated with the mean from the interval $[50,150]$, and standard deviation from $[30,60]$. Additionally, demand is randomly distributed among the surgery types $I$, and in case of semi-urgent surgery types also randomly distributed among the planning horizon $T$.

\subsubsection{Experimental results}

This section discusses the results of the different test instances that are evaluated with the CG model. To evaluate our CG model, we investigate the effect of varying the number of surgery types, surgery demand, and sub-specialties. Table 6.3 shows the effect of various surgery type demand on our heuristic solution value, lower bound, optimality gap, number of generated columns, and runtime.

From Table 6.3 we see that our approach is able to find solutions to both smaller and larger sized problems (regarding patient volume), in which 375 patients in total must be scheduled, just over 30 minutes in the largest case. When comparing the number of required ORs from our heuristic, and lower bounds 


\section{Chapter 6. Surgical procedure type scheduling incorporating semi urgent patients}

Table 6.3 Numerical results CG heuristic for varying surgery type demand

\begin{tabular}{|c|c|c|c|c|c|c|c|c|c|}
\hline \multirow[b]{2}{*}{$\mathrm{R}$} & \multicolumn{4}{|c|}{ Parameters } & \multicolumn{5}{|c|}{ Results } \\
\hline & $|S|$ & $|T|$ & $|I|$ & & $\mathrm{H}$ & LB & GAP & $\# \mathrm{col}$ & Time \\
\hline$\{240,480\}$ & 6 & 28 & 40 & 50,10 & 15 & 14.16 & 5.59 & 146 & 429 \\
\hline$\{240,480\}$ & 6 & 28 & 40 & 100,25 & 30 & 28.98 & 3.37 & 150 & 789 \\
\hline$\{240,480\}$ & 6 & 28 & 40 & 200,50 & 56.5 & 55.87 & 1.11 & 156 & 1331 \\
\hline$\{240,480\}$ & 6 & 28 & 40 & 300,75 & 85.5 & 83.43 & 2.42 & 140 & 1810 \\
\hline
\end{tabular}

(LB), we see that the heuristic solutions require zero to two (eight hour) ORs more in a four week planning horizon, and the optimality gap is at most $5.59 \%$, showing that the found solutions are of good quality. As more surgery types must be planned, the runtime roughly increases linearly, indicating that even very large numbers of surgeries may be planned within reasonable time.

Table 6.4 shows the effect of increasing the number of surgery types that may be scheduled in the planning horizon. Here we also see that the heuristic approach is able to find good solutions that at most require one OR too many, with an optimality gap of $3.43 \%$. Unlike an increase in the number of surgeries to plan, if there are more surgery types possible, the runtimes increase considerably faster. This is because more combinations of surgeries in an ORDS are possible, and thus more ORDS are generated that may improve the objective function. However, with 60 different surgery types the runtimes are still acceptable, from which we conclude that realistic instance sizes can be solved with our approach.

Table 6.4 Numerical results CG heuristic for varying surgery type numbers $|I|$

\begin{tabular}{|c|c|c|c|c|c|c|c|c|c|}
\hline \multirow[b]{2}{*}{$\mathrm{R}$} & \multicolumn{4}{|c|}{ Parameters } & \multicolumn{5}{|c|}{ Results } \\
\hline & $|S|$ & $|T|$ & $|I|$ & $\sum_{i} s_{i}$, & $\mathrm{H}$ & LB & GAP & $\# \mathrm{col}$ & Time \\
\hline$\{240,480\}$ & 6 & 14 & 30 & 100,25 & 28.5 & 28.07 & 1,51 & 117 & 277 \\
\hline$\{240,480\}$ & 6 & 14 & 40 & 100,25 & 31.5 & 30.51 & 3.14 & 187 & 544 \\
\hline$\{240,480\}$ & 6 & 14 & 50 & 100,25 & 30.5 & 29.45 & 3.43 & 275 & 1032 \\
\hline$\{240,480\}$ & 6 & 14 & 60 & 100,25 & 29 & 28.09 & 3.13 & 313 & 1225 \\
\hline
\end{tabular}

Table 6.5 shows the effect of increasing the number of surgical specialties to which surgery types may belong. Similar to the other test instances we find that good solutions are found using our heuristic. Additionally, the solution quality and run-time is not effected considerably when more surgical specialties are taken into account. As such, it may also be possible to use our approach on a per surgeon basis, instead of per surgical specialty and assign ORDS to individual surgeons.

\subsubsection{Case study data}

We apply our approach to a case study in a Dutch hospital. In this instance there are six sub-specialty types, who (together) carry out 47 different surgery 
Table 6.5 Numerical results CG heuristic for varying specialty numbers $|S|$

\begin{tabular}{lllllllllll}
\hline & \multicolumn{3}{c}{ Parameters } & \multicolumn{5}{c}{ Results } \\
$\mathrm{R}$ & $|S|$ & $|T|$ & $|I|$ & $\sum_{i} s_{i}, \sum_{i, t} \hat{s}_{i, t}$ & $\mathrm{H}$ & $\mathrm{LB}$ & GAP & $\#$ col & Time \\
\hline$\{240,480\}$ & 1 & 14 & 40 & 200,50 & 60 & 59.53 & 0.77 & 360 & 756 \\
$\{240,480\}$ & 3 & 14 & 40 & 200,50 & 60.5 & 60.15 & 0.56 & 257 & 755 \\
$\{240,480\}$ & 6 & 14 & 40 & 200,50 & 66 & 64.99 & 1.52 & 195 & 983 \\
$\{240,480\}$ & 9 & 14 & 40 & 200,50 & 62 & 61.16 & 1.35 & 152 & 1091 \\
$\{240,480\}$ & 12 & 14 & 40 & 200,50 & 61 & 58.98 & 3.30 & 139 & 1241 \\
\hline
\end{tabular}

types. We evaluate two scenarios, in which we schedule the minimal and average elective surgery demand (156 and 224 respectively) in addition to the semi-urgent surgeries. Of these surgery types, four are semi-urgent surgery types that are to be carried out within several days. The duration of all surgery types is assumed to be normally distributed and based on historical data. In addition, we assume that semi-urgent surgery demand arrives according to a Poisson distribution (based on historical data), specified for each day of the planning horizon and surgery type. The planning horizon in our case study is four weeks. Respectively there are at most six ORs, and two surgeons of each sub-specialty available to plan each (working) day of the week. Finally, we limit the overtime probability $\alpha$ to $30 \%$. Table 6.6 gives an overview of our case study, and Table 6.7 provides additional information on the semi-urgent surgery types.

\subsubsection{Case study results}

This section first describes the experiments to set the SAA input parameters. Thereafter, we apply our SAA model and CG based heuristic to our case study.

\section{SAA input parameters and results}

We apply the SAA method to different settings of $N(|N|=$ $5,10,25,50,100,200,500)$. In addition we use $|M|=10$ and $\left|N^{\prime}\right|=500$. Figure 6.2 shows the objective value behavior for different values of $|N|$. We find that from a sample size of 200, the objective function (14) upper and lower bound have converged, and confidence intervals around the bounds start to overlap.

As expected, the solution quality, and the computation time increases with the number of samples and replications. For our problem instance we found that a replication number of $|M|=10$, and sample size $|N|=200$ provided good results. We evaluate the effectiveness of the SAA model by comparing it to a deterministic expected value problem (EVP) version which includes a single average scenario that uses the expected values of the stochastic arrivals (i.e., using a single scenario $\zeta$ ). Figure 6.3 shows the total (weighted) waiting time of 100 iterations, each consisting of 100 simulated realizations of the EVP and SAA schedules in the planning horizon. We find that the schedule obtained by the 


\section{Chapter 6. Surgical procedure type scheduling incorporating semi urgent patients}

Table 6.6 Overview case study settings

\begin{tabular}{|c|c|c|}
\hline Set/Parameter & Description & Value \\
\hline$T$ & Days in planning horizon & $\{1, . ., 28\}$ \\
\hline$S$ & Sub-specialties & $\{1, \ldots, 6\}$ \\
\hline$I_{e}$ & Elective surgery types & $\{1, \ldots, 43\}$ \\
\hline$I_{u}$ & Semi-urgent surgery types & $\{44, \ldots, 47\}$ \\
\hline$J$ & Available operating rooms & $\{1, . ., 6\}$ \\
\hline$R$ & $\begin{array}{l}\text { Operating room size (opening } \\
\text { hours in minutes) }\end{array}$ & $\{240,360,480,540\}$ \\
\hline$e_{r}$ & $\begin{array}{l}\text { Cost of using an OR with } \\
\text { schedule of size } r\end{array}$ & $\{0.5,0.75,1,1.25\}$ \\
\hline$\alpha$ & $\begin{array}{l}\text { Maximum allowed overtime } \\
\text { probability }\end{array}$ & $30 \%$ \\
\hline$\hat{k}$ & $\begin{array}{l}\text { Maximum recorded waiting } \\
\text { time }\end{array}$ & 8 \\
\hline$\sum_{i} s_{i}$ & $\begin{array}{l}\text { Total demand of elective } \\
\text { surgery }\end{array}$ & 156,224 \\
\hline$\sum_{i} \sum_{t} \lambda_{i, t}$ & $\begin{array}{l}\text { Total (average) demand of } \\
\text { semi urgent surgery }\end{array}$ & 42.12 \\
\hline$\mu_{i}, \sigma_{i} ; i=28$ & $\begin{array}{l}\text { mean (minutes) and stdev of } \\
\text { shortest surgery type }\end{array}$ & $47.88,12.39$ \\
\hline$\mu_{i}, \sigma_{i} ; i=43$ & $\begin{array}{l}\text { mean (minutes) and stdev of } \\
\text { longest surgery type }\end{array}$ & $306.01,133.15$ \\
\hline
\end{tabular}

Table 6.7 Semi-urgent surgery information

\begin{tabular}{|c|c|c|c|c|c|c|c|}
\hline 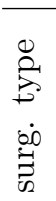 & 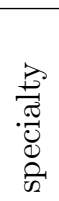 & 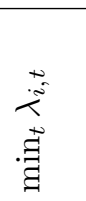 & 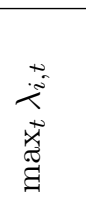 & $\begin{array}{c}2^{20} \\
\vdots \\
\vdots \\
0 \\
n^{2}\end{array}$ & 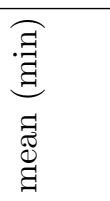 & $\frac{\vec{d}}{\vec{d}}$ & 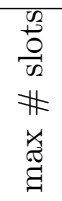 \\
\hline 44 & 1 & 0.33 & 0.93 & $\{1,2,3,4,5,50,60,70,80\}$ & 91.48 & 46.94 & 14 \\
\hline 45 & 2 & 0.12 & 0.36 & $\{1,2,3,4,5,50,60,70,80\}$ & 139.75 & 57.99 & 4 \\
\hline 46 & 6 & 0.21 & 0.65 & $\{1,2,3,4,5,50,60,70,80\}$ & 104.48 & 105.46 & 9 \\
\hline 47 & 5 & 0.41 & 1.22 & $\{1,2,3,4,5,50,60,70,80\}$ & 80.34 & 43.47 & 7 \\
\hline
\end{tabular}

SAA algorithm is better able to facilitate the uncertain arrivals of semi-urgent patients, resulting in less overall waiting time. Specifically, the average waiting time from the SAA is 1.79 days, and 2.89 days from the EVP. This makes sense, as in the EVP formulation only the average number of arrivals is accounted for. The model then assigns less surgery types in the planning horizon as these should be sufficient for the average case. The SAA model however, assigns more surgery types in the planning horizon to account for the stochasticity in the semi-urgent surgery demand in order to reduce waiting times. With the EVP, there are 49 semi-urgent types planned, and 58 with the SAA. In total, these additional 
Figure 6.2 SAA model objective value behaviour for different $|N|$

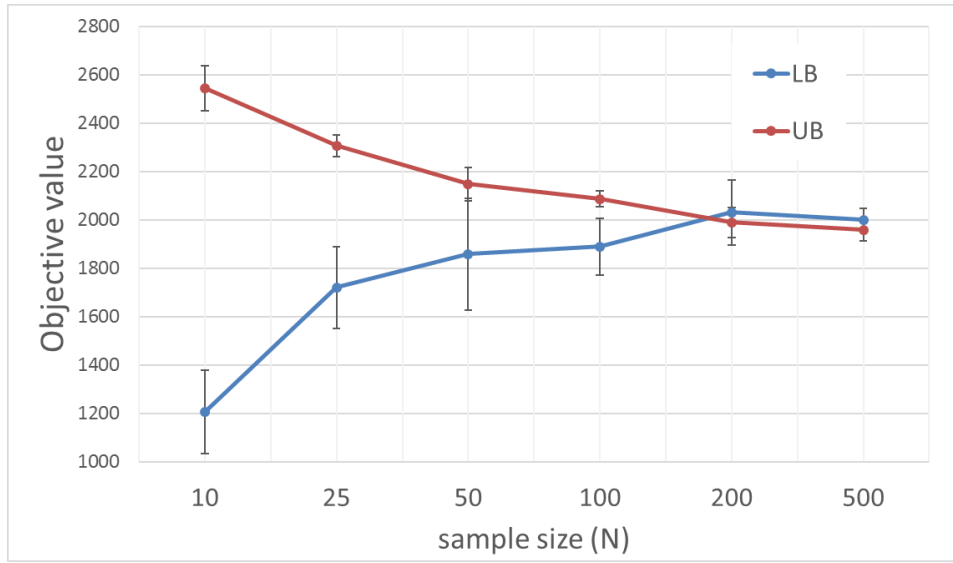

planned surgery types take up three ( 8 hour) ORs, which means that three more ORs are required during the planning period using the SAA model. Arguably however, in practice all semi-urgent surgery demand must be met. Thus, all semi-urgent surgery demand that may not be scheduled on time in allocated semi-urgent slots is scheduled by modifying the surgery type schedule (i.e., by rescheduling elective surgeries). With the SAA model the semi-urgent arrival stochasticity is better taken into account on a tactical level, and less disturbances would occur during the operational realization of the STS.

Figure 6.3 Simulation comparison of SAA and EVP schedules

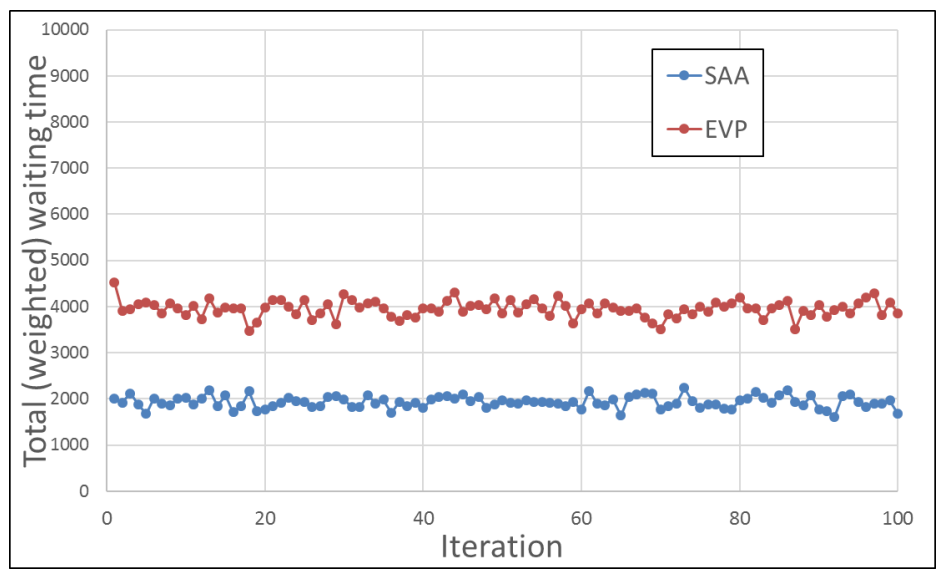

Table 6.8 lists the obtained semi-urgent surgery schedule which is used as input $\left(\hat{s}_{i, t}\right)$ in the column generation based heuristic in the next section. We find that semi-urgent surgeries are spread reasonably even through the planning horizon. This follows the current semi-urgent arrival distributions, which are assumed to be evenly distributed across the weeks in the planning horizon. 


\section{Chapter 6. Surgical procedure type scheduling incorporating semi urgent patients}

Table 6.8 Solution SAA model: assignment of semi-urgent surgeries to days in planning horizon

\begin{tabular}{|c|c|c|c|c|c|c|c|c|c|}
\hline$\hat{s}_{i, t}$ & $\mathrm{i}=44$ & $\mathrm{i}=45$ & $\mathrm{i}=46$ & $\mathrm{i}=47$ & $\hat{s}_{i, t}$ & $\mathrm{i}=44$ & $\mathrm{i}=45$ & $\mathrm{i}=46$ & $\mathrm{i}=47$ \\
\hline$\hat{s}_{i, 1}$ & 0 & 0 & 0 & 0 & $\hat{s}_{i, 15}$ & 0 & 0 & 0 & 1 \\
\hline$\hat{s}_{i, 2}$ & 0 & 0 & 0 & 0 & $\hat{s}_{i, 16}$ & 1 & 0 & 1 & 1 \\
\hline$\hat{s}_{i, 3}$ & 1 & 0 & 0 & 2 & $\hat{s}_{i, 17}$ & 1 & 1 & 1 & 1 \\
\hline$\hat{s}_{i, 4}$ & 1 & 1 & 1 & 1 & $\hat{s}_{i, 18}$ & 1 & 0 & 0 & 1 \\
\hline$\hat{s}_{i, 5}$ & 1 & 1 & 1 & 1 & $\hat{s}_{i, 19}$ & 1 & 1 & 1 & 2 \\
\hline$\hat{s}_{i, 6}$ & 0 & 0 & 0 & 0 & $\hat{s}_{i, 20}$ & 0 & 0 & 0 & 0 \\
\hline$\hat{s}_{i, 7}$ & 0 & 0 & 0 & 0 & $\hat{s}_{i, 21}$ & 0 & 0 & 0 & 0 \\
\hline$\hat{s}_{i, 8}$ & 1 & 0 & 0 & 1 & $\hat{s}_{i, 22}$ & 0 & 0 & 0 & 0 \\
\hline$\hat{s}_{i, 9}$ & 1 & 0 & 1 & 1 & $\hat{s}_{i, 23}$ & 1 & 0 & 1 & 2 \\
\hline$\hat{s}_{i, 10}$ & 1 & 1 & 1 & 1 & $\hat{s}_{i, 24}$ & 1 & 1 & 1 & 0 \\
\hline$\hat{s}_{i, 11}$ & 1 & 0 & 0 & 2 & $\hat{s}_{i, 25}$ & 1 & 0 & 0 & 2 \\
\hline$\hat{s}_{i, 12}$ & 1 & 1 & 1 & 1 & $\hat{s}_{i, 26}$ & 2 & 1 & 1 & 2 \\
\hline$\hat{s}_{i, 13}$ & 0 & 0 & 0 & 0 & $\hat{s}_{i, 27}$ & 0 & 0 & 0 & 0 \\
\hline$\hat{s}_{i, 14}$ & 0 & 0 & 0 & 0 & $\hat{s}_{i, 28}$ & 0 & 0 & 0 & 0 \\
\hline
\end{tabular}

\section{CG results}

We now apply the CG based heuristic to our case study. In addition, we evaluate the effect of using different surgery type schedule schedule sizes $r$, as well as varying levels of allowed overtime and elective surgery demand.

Besides only using full ORs ( 8 hours of operating time), we also investigate the influence of allowing shorter OR schedules. Additionally, we evaluate the effect on increasing operating room time by one hour (i.e., using 9 hour schedules). Note that OR costs are proportional to their size (e.g., a 4 hour OR costs half an 8 hour OR). Table 6.9 shows the results of the CG based approach applied to the case study data with varying OR schedule sizes and elective demand. We find that using our approach we are able to find a feasible solution in all cases within a reasonable time-frame. Comparing our approach to the regular formulation (from Section 6.3.1), the regular formulation was unable to obtain an integer solution after one hour of runtime. As expected, if elective demand of schedule size options increase, the runtime increases as well. In the case of more elective demand this is caused by additional iterations of our heuristic, as more surgery schedules must be fixed in the planning horizon to satisfy all demand. In the case of adding schedule sizes, the time in each column generation cycle increases, as pricing problems are solved for each subspecialty, day and schedule size combination $(s, t, r)$. In the largest settings our approach was able to find a feasible solution within 100 minutes, which should be acceptable given that new schedules and allocations have to be made at most once every planning horizon (i.e., once a month).

Regarding the optimality gap (defined as $(\mathrm{H}-\mathrm{LP}) / \mathrm{H})$ we find that as we allow more OR schedule sizes, the gap decreases, arguably as more surgery combinations become available and schedules may be created that better adapt to the 
Table 6.9 Results of CG based heuristic

\begin{tabular}{|c|c|c|c|c|c|c|c|}
\hline \multirow[t]{3}{*}{ Schedule sizes } & \multirow{3}{*}{$\begin{array}{l}\# \\
\text { (elec- } \\
\text { tive) } \\
\text { surg- } \\
\text { eries }\end{array}$} & \multicolumn{4}{|c|}{ Obj.Val. Obj.Val. Opt.Gap \# } & \multicolumn{2}{|c|}{ Runtime \# Used } \\
\hline & & $\mathrm{H}$ & $\mathrm{LP}$ & $(\%)$ & \multicolumn{2}{|c|}{ Gen.Col. (sec) } & \multirow[t]{2}{*}{ Col. } \\
\hline & & & & & & & \\
\hline$\{480\}$ & 156 & 69.00 & 61.74 & 10.52 & 132 & 898 & 33 \\
\hline$\{540\}$ & 156 & 74.25 & 60.46 & 18.57 & 180 & 1024 & 33 \\
\hline$\{240,480\}$ & 156 & 60.50 & 56.00 & 7.43 & 176 & 1671 & 35 \\
\hline$\{360,480\}$ & 156 & 59.75 & 54,71 & 8.43 & 223 & 1983 & 38 \\
\hline$\{240,360,480\}$ & 156 & 57.25 & 54,59 & 4.63 & 262 & 3742 & 38 \\
\hline$\{480\}$ & 224 & 83.00 & 76.77 & 7.49 & 155 & 1538 & 43 \\
\hline$\{540\}$ & 224 & 84.37 & 75.79 & 10.10 & 191 & 1737 & 33 \\
\hline$\{240,480\}$ & 224 & 76.50 & 73.87 & 3.44 & 221 & 2797 & 47 \\
\hline$\{360,480\}$ & 224 & 76.25 & 72.73 & 4.61 & 249 & 3112 & 42 \\
\hline$\{240,360,480\}$ & 224 & 75.00 & 72.73 & 3.02 & 281 & 5527 & 45 \\
\hline
\end{tabular}

elective and semi-urgent demand. In the current practice where only 8 hour schedules are used, our approach has an optimality gap of $10.5 \%$, which in practice means during the planned month at most 7 ORs too many are scheduled. This gap decreases to just under $5 \%$ when allowing 4 and 6 hour schedules. When more schedule sizes are allowed the overall required number of ORs decreases. For example, when 4 hours schedules are allowed in addition to 8 hour schedules, the objective value decreases by 8.5 . This means that by also using smaller schedules, the same surgery demand can be met with 8.5 ( 8 hour) ORs fewer. This OR capacity in turn could be allocated dynamically to to other surgical specialties that may require additional capacity based on patient waiting lists, and may allow the same hospital surgical demand be met with fewer required ORs.

In addition to the evaluation of different schedule sizes we also evaluate the effect of the overtime constraint on the required number of ORs during the planning horizon. Figure 6.4 shows the number of required ORs for various overtime probabilities for two cases $(R=\{480\}$ and $\{240,480\})$. Note that a $50 \%$ overtime probability corresponds to no additional slack inclusion when creating surgery schedules (i.e., plan using average surgery times). As expected, when less overtime is allowed, more operating rooms are needed as more slack is incorporated per OR. There are however diminishing returns when only 8 hour schedules are used, where increasing accepted overtime from $5 \%$ to $20 \%$ reduces the number of required ORs by 8 , and a further increase to $40 \%$ only saves two additional ORs. In contrast, if 4 and 8 hour schedules are used the required number of ORs keeps decreasing as acceptable overtime probabilities increase. High overtime probability in practice could lead to both patient and staff dissatisfaction, as ORs have long working hours, and patients must wait considerably longer during the day before their surgery starts, or in worse cases may even be cancelled. 


\section{Chapter 6. Surgical procedure type scheduling incorporating semi urgent patients}

Figure 6.4 Comparison of required number of ORs for overtime probabilities

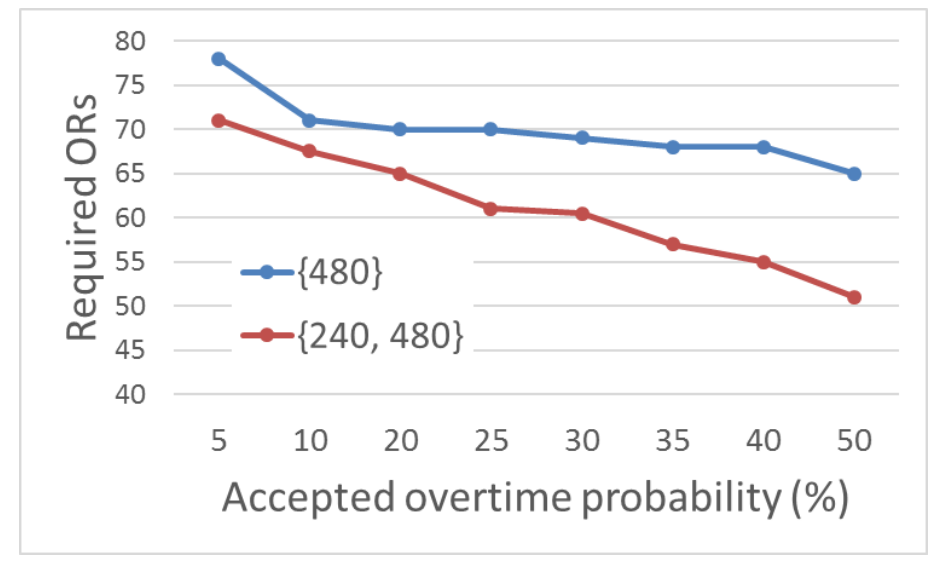

For the hospital setting under consideration, it does not seem cost efficient on a per hour basis to extend operating room times (and thus schedules) by one hour. While there is a reduction in the largest setting of 8 ORDS (i.e., 64 hours) required in the month, the 75 scheduled ORDS each take an additional hour such that overall more time is required to meet the same surgery demand. Thus, simply assigning more capacity does not necessarily yield improvements. Conversely, when also scheduling 4 hour ORDS, which seem most likely from an organizational perspective, a reduction of 6.5 (full) ORs can be achieved while still meeting surgery demand. This suggests it may be worthwhile to investigate the possibility of allocating half day OR blocks to specialties, such that OR capacity may be better distributed across surgical specialties and more demand may be met. Additionally, in the current setting of using full day ORDS, the overtime probability may be considerably reduced $(30 \%$ to $10 \%)$ at the cost of two additional ORs per month which may be desirable from a patient and staff perspective. Finally, using this approach to create surgery type schedules may benefit the operational level surgical case assignment. By assigning surgical cases to the STS blueprint on a first come first serve basis, patients may be scheduled more quickly, and without as much (re)scheduling to fit patients into ORs, as this is done (close to) optimally in the STS. In summary, we find that our heuristic approach is able to find feasible solutions for all settings that are also of high quality. Especially if multiple schedule sizes are allowed, our approach is able to find near optimal solutions efficiently.

\subsection{Conclusion}

This study considers a tactical operating room planning problem in which not only elective, but also semi-urgent surgeries must be scheduled. We take the stochastic nature of surgery durations, and demand for semi-urgent surgeries into account explicitly. To solve this planning problem a stochastic model consider- 
ing uncertain semi-urgent arrivals and surgery durations is formulated. Following this, we adopt a decomposition approach wherein first SAA is used to allocate semi-urgent surgery capacity to days in the planning horizon. Second, a surgical type schedule is constructed using a CG based heuristic approach, in which elective and semi-urgent surgeries are combined into OR schedules and placed in the planning horizon. In this approach the number of required operating rooms is minimized, while ensuring the probability of overtime is limited.

Applying our approach to a case study we find that incorporating the stochastic nature of semi-urgent surgery demand reduces waiting times for semi-urgent patients. Also, with our CG based heuristic we are able to create surgery type schedules within a reasonable time. For our case study, when comparing our solution to the lower bound obtained by the (first) CG iteration we find that our solution is within $11 \%$ of the true optimal solution, and this improves to less than $5 \%$ if more OR schedule sizes are allowed. In addition, when allowing for different OR sizes (i.e., 4 and 6 hour ORs) considerably less ORs are required to schedule all surgery types.

In practice our approach may be used to schedule both the elective and semiurgent patients for a medium term planning horizon. The semi-urgent surgery requests may be based on historical data and updated accordingly. In addition, based on current waiting lists the number of elective surgeries that must be scheduled within the planning horizon may be changed. By including semi-urgent surgeries within the planning problem, less rescheduling of electives takes place as inevitably semi-urgent surgery requests arrive.

In our current application we assumed emergency surgeries (i.e., patients that must be treated on the day of arrival) are treated in dedicated emergency ORs. An extension to our approach would be to include these surgery types as well, by incorporating additional slack for emergencies. In addition, we currently do not sequence surgery types during a surgery type schedule (i.e., determine the order of surgeries), which may influence emergency patient waiting times. By considering sequencing when including emergency patients in the planning problem, further improvements may be achieved.

We assumed the arrival rates of semi-urgent surgeries to be fixed. In practice however, these patients are often added to the waiting list after consultation hours in which they consult with surgeons. Our planning approach however allocates ORDS to the planning horizon that require a surgeon, which in turn may change the days surgeons are available for consultations with patients. A possible future direction would be including the effect of the surgery type schedule on consultation hours, and thus semi-urgent arrival rates.

Besides the inclusion of different surgery type schedule sizes, allowing ORs to be used during weekends may benefit semi-urgent patients. Arguably, allowing surgeries to be planned during weekends allows the schedule to better fit to semiurgent patient arrivals. Of interest would be further investigation of the trade-off between waiting time improvements and additional costs incurred during the weekend. Finally, our heuristic approach results in a approximate solution, and 
Chapter 6. Surgical procedure type scheduling incorporating semi urgent patients

investigating possibilities of exactly solving our planning problems may result in improvements. For example, applying a branch and price approach may be investigated along with various branching strategies. 


\section{Conclusion and outlook}

The demand for the timely delivery of high quality urgent care is increasing. Combined with the uncertainties that arise during the delivery of care, and high stakes involved when urgent patients are concerned, this puts pressure on hospitals to efficiently and effectively organize healthcare processes that may adapt to the specific needs and characteristics of urgent care. This dissertation presents methodologies and models that can assist hospitals in effective urgent care delivery, while also efficiently utilizing resources. In this final chapter we present our main conclusions and give an outlook on future research.

In Chapter 2 we have explored the literature on the planning and control of non-elective care delivery in hospitals, within and across hospital departments. We have presented a structured overview of the various problems encountered when offering non-elective care across the entire patient pathway. We have discussed the results found in the literature regarding how to manage non-elective care processes, and have identified research opportunities. There have been studies focusing on all hospital departments, with the main focus on emergency department (ED) and Wards. There have been studies focusing on all hierarchical levels of control, with the main focus on the tactical level. Computer simulation and mathematical programming are the most used mathematical modeling approaches. We found that many of the results are hard to generalize, as they are based on a specific case study. Also, very few studies actually report about (successful) implementation of the outcomes. The diversity of treatment targets for urgent care patient types varies greatly across papers, particularly in ED and OR studies. Also, very few papers account for priority changing, which is realistic in practice. Finally, we identify that there is a great need for (generalizable) benchmark instance sets. Many studies focus on very similar problems, in different case studies.

One of the first departments faced by patients with an urgent demand for care is the ED. In Chapter 3 we study an ED and general practitioner's post (GPP) that have integrated into a single point emergency post as a point of access for acute care. Using computer simulation and a heuristic approach we sought the best allocation of resources and process design when combining these departments. From Chapter 3 we find that the merging of departments offers numerous possibilities for improvement through cooperation. A potential future research topic is the modeling of interaction between the integrated emergency 
post and other departments in the hospital, such that the entire care pathway chain can be optimized.

One place where elective and non-elective patient pathways cross is the diagnostics department. Here, we find there is a trade-off between offering sufficient appointments for elective patients, and reserving sufficient capacity for non-elective patients to ensure short waiting times. In Chapter 4 we optimized appointment schedules of CT scanners for elective patients that influence elective patients' access time, elective and non-elective waiting time, and staff overtime. We find that by taking into account non-elective patient characteristics (arrival and duration) within both the diagnostics department and the ED, the diagnostics appointment schedule may be changed such that performance for all patients involved improves. Also, it results in a more even workload during the day. Aside from diagnostics requests from the ED, part of the unscheduled diagnostic requests originate from other departments such as the wards. It may be interesting to include additional processes and resources that affect the appointment planning, for example by simultaneously (re)scheduling the patient rounds on wards, and thus better align patient flows across wards, diagnostics, and ED. Another possibility for future research is the incorporation of various priority rules of patients, investigating the effect on the appointment schedules and patient flows.

In Chapter 5 we study multiple policies for operating theaters to allocate operating room (OR) capacity to emergency patients, such as the use of dedicated emergency ORs. We identify the patient and hospital characteristics that have a large effect on the effectiveness of policies, and conclude that there is no approach that works best in all situations. Additionally, Chapter 5 provides an evaluation of policies for numerous settings (of patient and hospital characteristics) such that hospitals may find the policy configuration best for their setting. These evaluations have been integrated in an interactive tool, which has already been used by at least one Dutch hospital to improve their policy. A promising direction for future research is to develop policies which may offer viable alternatives to those currently used in practice. An example of such a policy is one where at any moment in time one elective OR is the emergency OR. Its elective program is put 'on hold', until the completion of a surgery in another elective OR that will then take over the emergency OR role. Consequently, the role of 'emergency OR' jumps between elective ORs. In this way, an emergency patient can always be immediately serviced upon arrival.

Many non-elective patients may wait for some time to be serviced. Unlike emergency patients that must be treated on the day of arrival, such so-called semi-urgent surgeries may be postponed for some days. If not accounted for, adjustments of the existing elective schedule may be required to plan these semiurgent patients. In Chapter 6 we incorporate both elective and semi-urgent patients in a surgery type schedule blueprint and find that doing so can improve OR utilizations, and lead to less rescheduling of electives. Given the uncertain arrivals of semi-urgent and emergency patients, the realized schedule may still 
need to be adapted. A possibility for future research would be to incorporate our methodology into a rolling horizon approach that is constantly updated, and takes into account the current status of the operating rooms, including for example the waiting lists.

Another possibility for future research is the dynamic allocation and design of processes. Due to the inherent variability in non-elective care, there is constant change at departments. It may not necessarily be the case that a specific patient pathway design or resource allocation is best regardless of the (current) state of the system. For example, the use of fast-track pathways at the ED may not always be useful in alleviating ED waiting times. Further research on the dynamic design of processes and (re)allocation of resources may offer valuable improvements and allow hospitals to better adapt to both short- and long term changes.

Summarizing, the work presented in this dissertation and the directions for future research may assist hospitals in decision making with regards to the planning and control of non-elective care processes. This ensures that patients are treated effectively, in a timely manner, and hospital resources are efficiently utilized. This dissertation gives several examples, where the uncertain aspects of various types of non-elective care may be included in the modeling of processes in order to improve outcomes for all patients involved. 



\section{Bibliography}

[1] W. Abo-Hamad and A. Arisha. Simulation-based framework to improve patient experience in an emergency department. European Journal of Operational Research, 224(1):154-166, 2013.

[2] I. Adan, J. Bekkers, N. Dellaert, J. Jeunet, and J. Vissers. Improving operational effectiveness of tactical master plans for emergency and elective patients under stochastic demand and capacitated resources. European Journal of Operational Research, 213(1):290-308, 2011.

[3] M. A. Ahmed and T. M. Alkhamis. Simulation optimization for an emergency department healthcare unit in kuwait. European Journal of Operational Research, 198(3):936-942, 2009.

[4] S. Ahmed and A. Shapiro. The sample average approximation method for stochastic programs with integer recourse. Technical report (available at: http://www2.isye.gatech.edu/ sahmed/), Georgia Institute of Technology, Atlanta, GA, 2002.

[5] R. Alvarez, G. A. Sandoval, S. Quijada, and A. D. Brown. A simulation study to analyze the impact of different emergency physician shift structures in an emergency department. In Proceedings of the 35th International Conference on Operational Research Applied to Health Services (ORAHS), Leuven, Belgium, 2009.

[6] S. Andradóttir. A review of simulation optimization techniques. In Proceedings of the Winter Simulation Conference, pages 151-158, Washington, D.C., USA, 1998.

[7] S. Andradóttir. Chapter 20 an overview of simulation optimization via random search. In S. G. Henderson and B. L. Nelson, editors, Simulation, volume 13 of Handbooks in Operations Research and Management Science, pages 617 - 631. Elsevier, 2006.

[8] J. M. O. Antognini, J. F. Antognini, and V. Khatri. How many operating rooms are needed to manage non-elective surgical cases? a monte carlo simulation study. BMC Health Services Research, 15(1):487, 2015.

[9] J. April, F. Glover, J. P. Kelly, and M. Laguna. Simulation-based optimization: practical introduction to simulation optimization. In Proceedings of the Winter Simulation Conference, pages 71-78, New Orleans, USA, 2003.

[10] N. T. Argon, S. Ziya, and R. Righter. Scheduling impatient jobs in a clearing system with insights on patient triage in mass casualty incidents. Probability in the Engineering and Informational Sciences, 22(3):301-332, 2008. 
[11] R. Aringhieri, M. Bruni, S. Khodaparasti, and J. van Essen. Emergency medical services and beyond: Addressing new challenges through a wide literature review. Computers 63 Operations Research, 78:349 - 368, 2017.

[12] O. M. Ashour and G. E. O. Kremer. A simulation analysis of the impact of fahp-maut triage algorithm on the emergency department performance measures. Expert Systems with Applications, 40(1):177-187, 2013.

[13] O. M. Ashour and G. E. O. Kremer. Dynamic patient grouping and prioritization: a new approach to emergency department flow improvement. Health Care Management Science, 19(2):192-205, 2016.

[14] L. Au, G. B. Byrnes, C. A. Bain, M. Fackrell, C. Brand, D. A. Campbell, and P. G. Taylor. Predicting overflow in an emergency department. IMA Journal of Management Mathematics, 20(1):39, 2009.

[15] Australasian College for Emergency Medicine. Australasian college for emergency medicine: Guidelines on the implementation of the australasian triage scale in emergency departments. available at: http://www.acem.org.au/, 2016.

[16] A. Azadeh, M. H. Farahani, S. Torabzadeh, and M. Baghersad. Scheduling prioritized patients in emergency department laboratories. Computer methods and programs in biomedicine, 117(2):61-70, 2014.

[17] A. Azadeha, H. Tohidi, M. Zarrin, S. Pashapour, and M. Moghaddam. An integrated algorithm for performance optimization of neurosurgical ICUs. Expert Systems with Applications, 43:142-153, 2016.

[18] S. Azari-Rad, A. Yontef, D. M. Aleman, and D. R. Urbach. A simulation model for perioperative process improvement. Operations Research for Health Care, 3 (1):22-30, 2014.

[19] A. E. Bair, W. T. Song, Y. C. Chen, and B. A. Morris. The impact of inpatient boarding on ed efficiency: A discrete-event simulation study. Journal of Medical Systems, 34(5):919-929, 2010.

[20] R. R. Barton and M. Meckesheimer. Chapter 18 metamodel-based simulation optimization. In S. G. Henderson and B. L. Nelson, editors, Simulation, volume 13 of Handbooks in Operations Research and Management Science, pages 535 - 574 . Elsevier, 2006.

[21] A. Başar, B. Çatay, and T. Ünlüyurt. A taxonomy for emergency service station location problem. Optimization Letters, 6(6):1147-1160, 2012.

[22] S. Bayer, C. Petsoulas, B. Cox, A. Honeyman, and J. Barlow. Facilitating stroke care planning through simulation modelling. Health Informatics Journal, 16(2): 129-143, 2010.

[23] E. Beck, H. Balasubramanian, P. L. Henneman, and Ieee. Resource management and process change in a simplified model of the emergency department. In Proceedings of the Winter Simulation Conference, pages 1838-1846, Austin, USA, 2009 . 
[24] R. Ben Bachouch, A. Guinet, and S. Hajri-Gabouj. An integer linear model for hospital bed planning. International Journal of Production Economics, 140(2): 833-843, 2012.

[25] B. Bettonvil and J. P. Kleijnen. Searching for important factors in simulation models with many factors: Sequential bifurcation. European Journal of Operational Research, 96(1):180 - 194, 1997.

[26] T. Bhattacharyya, M. S. Vrahas, S. M. Morrison, E. Kim, R. A. Wiklund, R. M. Smith, and H. E. Rubash. The value of the dedicated orthopaedic trauma operating room. Journal of Trauma-injury Infection and Critical Care, 60(6):1336-1340, June 2006.

[27] J. Bisschop. AIMMS Modeling Guide. (available at: https://aimms.com/english/developers/resources/), Haarlem, Netherlands, 2016 .

[28] J. Boesel, B. L. Nelson, and S.-H. Kim. Using ranking and selection to clean up after simulation optimization. Operations Research, 51(5):814-825, 2003.

[29] N. J. Borgman, M. R. K. Mes, I. M. H. Vliegen, and E. W. Hans. Improving the design and operation of an integrated emergency post via simulation. Journal of Simulation, 9(2):99-110, 2015.

[30] N. J. Borgman, I. M. H. Vliegen, and E. Hans. Emergency or or not? a formal analysis of emergency surgery scheduling policies. Working paper, 2017.

[31] J. J. Bosch. Better utilisation of the or with less beds: a tactical surgery scheduling approach to improve or utilisation and the required number of beds in the wards. Master's thesis, University of Twente, Netherlands, 2011.

[32] J. Bowers and G. Mould. Managing uncertainty in orthopaedic trauma theatres. European Journal of Operational Research, 154(3):599 - 608, 2004.

[33] S. C. Brailsford, V. A. Lattimer, P. Tarnaras, and J. C. Turnbull. Emergency and on-demand health care: modelling a large complex system. Journal of the Operational Research Society, 55(1):34-42, 2004.

[34] S. C. Brailsford, P. R. Harper, B. Patel, and M. Pitt. An analysis of the academic literature on simulation and modelling in health care. Journal of Simulation, 3 (3):130-140, 2009.

[35] C. Brasted. Ultrasound waiting lists: rational queue or extended capacity? a study of the mechanisms and diverse realities underlying ultrasound waiting lists. Health Care Management Science, 11(2):196-207, 2008.

[36] S. Brenner, Z. Zeng, Y. Liu, J. Wang, J. Li, and P. K. Howard. Modeling and analysis of the emergency department at university of kentucky chandler hospital using simulations. Journal of Emergency Nursing, 36(4):303-310, 2010.

[37] K. M. Bretthauer, H. S. Heese, H. Pun, and E. Coe. Blocking in healthcare operations: A new heuristic and an application. Production and Operations Management, 20(3):375-391, 2011. 
[38] L. Brotcorne, G. Laporte, and F. Semet. Ambulance location and relocation models. European Journal of Operational Research, 147(3):451 - 463, 2003.

[39] M. Cabrera, Eduardo; Taboada, M. L. Iglesias, F. Epelde, and E. Luque. Optimization of healthcare emergency departments by agent-based simulation. Procedia Computer Science, 4(0):1880-1889, 2011.

[40] B. Cardoen, E. Demeulemeester, and J. Belin. Operating room planning and scheduling: A literature review. European Journal of Operational Research, 201 (3):921 - 932, 2010.

[41] T. Cayirli and E. Veral. Outpatient scheduling in health care: a review of literature. Production and Operations Management, 12(4):519-549, 2003.

[42] T. Cayirli, E. Veral, and H. Rosen. Designing appointment scheduling systems for ambulatory care services. Health Care Management Science, 9(1):47-58, 2006.

[43] T. Cayirli, E. Veral, and H. Rosen. Assessment of patient classification in appointment system design. Production and Operations Management, 17(3):338$353,2008$.

[44] R. Ceglowski, L. Churilov, and J. Wasserthiel. Combining data mining and discrete event simulation for a value-added view of a hospital emergency department. Journal of the Operational Research Society, 58(2):246-254, 2007.

[45] C. W. Chan, V. F. Farias, N. Bambos, and G. J. Escobar. Optimizing intensive care unit discharge decisions with patient readmissions. Operations Research, 60 (6):1323-1341, 2012.

[46] R. C. H. Cheng. Searching for important factors: Sequential bifurcation under uncertainty. In Proceedings of the Winter Simulation Conference, pages 275-280, Atlanta, USA, 1997.

[47] A. Chockalingam, K. Jayakumar, and M. A. Lawley. A stochastic control approach to avoiding emergency department overcrowding. In B. Johansson, S. Jain, J. MontoyaTorres, J. Hugan, and E. Yucesan, editors, Proceedings of the Winter Simulation Conference, pages 2399-2411, Baltimore, USA, 2010.

[48] J. K. Cochran and K. T. Roche. A multi-class queuing network analysis methodology for improving hospital emergency department performance. Computers $\mathbb{E}$ Operations Research, 36(5):1497-1512, 2009.

[49] P. Congdon. The development of gravity models for hospital patient flows under system change: a bayesian modelling approach. Health Care Management Science, 4(4):289-304, 2001.

[50] A. X. Costa, S. A. Ridley, A. K. Shahani, P. R. Harper, V. De Senna, and M. S. Nielsen. Mathematical modelling and simulation for planning critical care capacity. Anaesthesia, 58:320-327, 2003.

[51] E. A. Crawford, P. J. Parikh, N. Kong, and C. V. Thakar. Analyzing discharge strategies during acute care: A discrete-event simulation study. Medical Decision Making, 34(2):231-241, 2014. 
[52] M. Criswell, I. Hasan, R. Kopach, S. Lambert, M. Lawley, D. McWilliams, G. Trupiano, and N. Varadarajan. Emergency department divert avoidance using petri nets. IEEE International Conference on System of Systems Engineering, pages $1-6,2007$.

[53] D. Daldoul, I. Nouaouri, H. Bouchriha, and H. Allaoui. Optimization on human and material resources in emergency department. 2015 International Conference on Industrial Engineering and Systems Management (Iesm), pages 633-638, 2015.

[54] N. P. Dellaert and J. Jeunet. Hospital admission planning to optimize major resources utilization under uncertainty. Beta working paper; vol. 319 (available at: http://repository.tue.nl/), Eindhoven University of Technology, Eindhoven, Netherlands, 2010.

[55] M. Diefenbach and E. Kozan. Effects of bed configurations at a hospital emergency department. Journal of Simulation, 5(1):44-57, 2011.

[56] C. J. M. Doggen, E. W. Hans, J. E. Snel, D. V. Velde, and H. J. W. Verheij. Subsidieaanvraag: "optimale logistiek en patienten voorkeuren in de acute zorgketen; de huisartsenpost en spoedeisende hulpin een geintegreerde spoedpost" [in dutch]. Research Proposal (available at: http://zonmw.nl), 2010.

[57] C. Duguay and F. Chetouane. Modeling and improving emergency department systems using discrete event simulation. Simulation, 83(4):311-320, 2007.

[58] O. El-Rifai, T. Garaix, V. Augusto, and X. L. Xie. A stochastic optimization model for shift scheduling in emergency departments. Health Care Management Science, 18(3):289-302, 2015.

[59] A. Elalouf and G. Wachtel. An alternative scheduling approach for improving emergency department performance. International Journal of Production Economics, 178:65-71, 2016.

[60] S. G. Elkhuizen, J. R. van Sambeek, E. W. Hans, K. J. Krabbendam, and P. J. Bakker. Applying the variety reduction principle to management of ancillary services. Health Care Management Review, 32(1):37-45, 2007.

[61] E. Erdem, X. L. Qu, and J. Shi. Rescheduling of elective patients upon the arrival of emergency patients. Decision Support Systems, 54(1):551-563, 2012.

[62] H. Eskandari, M. Riyahifard, S. Khosravi, and C. D. Geiger. Improving the emergency department performance using simulation and mcdm methods. In S. Jain, R. Creasey, and J. Himmelspach, editors, Proceedings of the Winter Simulation Conference, pages 1211-1222, Phoenix, USA, 2011.

[63] J. T. van Essen, J. L. Hurink, W. Hartholt, and B. J. van den Akker. Decision support system for the operating room rescheduling problem. Health Care Management Science, 15(4):355-372, 2012.

[64] J. van Essen, E. Hans, J. Hurink, and A. Oversberg. Minimizing the waiting time for emergency surgery. Operations Research for Health Care, 1(23):34 - 44, 2012. 
[65] N. Farrohknia, M. Castrén, A. Ehrenberg, L. Lind, S. Oredsson, H. Jonsson, K. Asplund, and K. E. Göransson. Emergency department triage scales and their components: A systematic review of the scientific evidence. Scandinavian Journal of Trauma, Resuscitation and Emergency Medicine, 19(1):42, 2011.

[66] H. Fei, C. Chu, N. Meskens, and A. Artiba. Solving surgical cases assignment problem by a branch-and-price approach. International Journal of Production Economics, 112(1):96 - 108, 2008.

[67] H. Fei, C. Chu, and N. Meskens. Solving a tactical operating room planning problem by a column-generation-based heuristic procedure with four criteria. Annals of Operations Research, 166(1):91, 2009.

[68] Y. Ferrand, M. Magazine, and U. Rao. Comparing two operating-room-allocation policies for elective and emergency surgeries. In B. Johansson, S. Jain, J. MontoyaTorres, J. Hugan, and E. Yucesan, editors, Proceedings of the Winter Simulation Conference, pages 2364-2374, Baltimore, USA, 2010.

[69] Y. B. Ferrand, M. J. Magazine, and U. S. Rao. Partially flexible operating rooms for elective and emergency surgeries. Decision Sciences, 45(5):819-847, October 2014.

[70] Y. B. Ferrand, M. J. Magazine, and U. S. Rao. Managing operating room efficiency and responsiveness for emergency and elective surgeriesa literature survey. IIE Transactions on Healthcare Systems Engineering, 4(1):49-64, 2014.

[71] D. M. Ferrin, M. J. Miller, and D. L. McBroom. Maximizing hospital finanacial impact and emergency department throughput with simulation. In Proceedings of the Winter Simulation Conference, pages 1566-1573, Washington D.C., USA, 2007.

[72] E. B. Fields, G. E. Okudan, and O. M. Ashour. Rank aggregation methods comparison: A case for triage prioritization. Expert Systems with Applications, 40(4):1305-1311, 2013.

[73] M. Findlay and H. Grant. An application of discrete-event simulation to an outpatient healthcare clinic with batch arrivals. In S. Jain, R. Creasey, and J. Himmelspach, editors, Proceedings of the Winter Simulation Conference, pages 1166-1177, Phoenix, USA, 2011.

[74] A. Fletcher, D. Halsall, S. Huxham, and D. Worthington. The dh accident and emergency department model: a national generic model used locally. Journal of the Operational Research Society, 58(12):1554-1562, 2007.

[75] D. Fone, S. Hollinghurst, M. Temple, A. Round, N. Lester, A. Weightman, K. Roberts, E. Coyle, G. Bevan, and S. Palmer. Systematic review of the use and value of computer simulation modelling in population health and health care delivery. Journal of Public Health, 25(4):325-335, 2003.

[76] C. Fransman. Patient and community preferences for out-of-hours emergency care using best-worst scaling. Msc thesis, University of Twente, Netherlands, 2011. 
[77] N. K. Freeman, S. H. Melouk, and J. Mittenthal. A scenario-based approach for operating theater scheduling under uncertainty. Manufacturing \& S Service Operations Management, 18(2):245-261, 2016.

[78] M. C. Fu, F. W. Glover, and J. April. Simulation optimization: a review, new developments, and applications. In Proceedings of the Winter Simulation Conference, pages 13 pp.-, Orlando, USA, Dec 2005.

[79] P. H. P. Fung Kon Jin, M. G. W. Dijkgraaf, C. L. Alons, C. van Kuijk, L. F. M. Beenen, G. M. Koole, and J. C. Goslings. Improving ct scan capabilities with a new trauma workflow concept: Simulation of hospital logistics using different ct scanner scenarios. European Journal of Radiology, 80(2):504-509, 2011.

[80] N. Gilboy, P. Tanabe, D. Travers, D. Eitel, and R. Wuerz. The emergency severity index. Agency for healthcare research and quality, Handbook (available at: https://www.ahrq.gov/), 2011.

[81] W. G. Gnanlet, Adelina; Gilland. Sequential and simultaneous decision making for optimizing health care resource flexibilities. Decision Sciences, 40(2):295-326, 2009 .

[82] J. B. Goldberg. Operations research models for the deployment of emergency services vehicles. EMS Management Journal, 1(1):20-39, 2004.

[83] L. V. Green. How many hospital beds? Inquiry-the Journal of Health Care Organization Provision and Financing, 39(4):400-412, 2002.

[84] L. V. Green, S. Savin, and B. Wang. Managing patient service in a diagnostic medical facility. Operations Research, 54(1):11-25, 2006.

[85] L. V. Green, J. Soares, J. F. Giglio, and R. A. Green. Using queueing theory to increase the effectiveness of emergency department provider staffing. Academic Emergency Medicine, 13(1):61-8, 2006.

[86] L. V. Green and P. J. Kolesar. Anniversary article: Improving emergency responsiveness with management science. Management Science, 50(8):1001-1014, 2004 .

[87] J. D. Griffiths, V. Knight, and I. Komenda. Bed management in a critical care unit. IMA Journal of Management Mathematics, 24(2):137-153, 2013.

[88] R. Grol, P. Giesen, and C. van Uden. After-hours care in the united kingdom, denmark, and the netherlands: New models. Health Affairs, 25(6):1733-1737, 2006 .

[89] F. Guerriero and R. Guido. Operational research in the management of the operating theatre: a survey. Health Care Management Science, 14(1):89-114, 2011.

[90] M. M. Gunal and M. Pidd. Interconnected DES models of emergency, outpatient, and inpatient departments of a hospital. In Proceedings of the Winter Simulation Conference, pages 1440-1445, Washington D.C., USA, 2007. 
[91] M. M. Gunal and M. Pidd. Discrete event simulation for performance modelling in health care: a review of the literature. Journal of Simulation, 4(1):42-51, 2010.

[92] H. A. Guo and J. F. Tang. Integrating simulation with optimization in emergency department management. In P. W. Tse, J. Mathew, K. Wong, R. Lam, and C. N. Ko, editors, Engineering Asset Management - Systems, Professional Practices and Certification, Lecture Notes in Mechanical Engineering, pages 1483-1496. Springer-Verlag Berlin, Berlin, 2015.

[93] D. Gupta, M. K. Natarajan, A. Gafni, L. Wang, D. Shilton, D. Holder, and S. Yusuf. Capacity planning for cardiac catheterization: A case study. Health Policy, 82(1):1-11, 2007.

[94] D. Gupta and B. Denton. Appointment scheduling in health care: Challenges and opportunities. IIE transactions, 40(9):800-819, 2008.

[95] HagaZiekenhuis. Facts and figures 2015. Report (available at: https://www.hagaziekenhuis.nl/over-hagaziekenhuis/verslaglegging-enverantwoording/), 2016.

[96] HagaZiekenhuis. Over hagaziekenhuis. zorgzaamheid, innovatie en samenwerking [in Dutch]. Retrieved March 10, 2017 (available at: https://www.hagaziekenhuis.nl/over-hagaziekenhuis/), 2017.

[97] S. Hahn-Goldberg, E. Chow, E. Appel, F. T. F. Ko, P. Tan, M. B. Gavin, T. Ng, H. B. Abrams, L. K. Casaubon, and M. W. Carter. Discrete event simulation of patient admissions to a neurovascular unit. Journal of Healthcare Engineering, 5 (3):347-359, 2014.

[98] S. Hajer and B. Mounir. Study in performance analyses of hospital emergency services: Case habib bourguiba hospital. In 2013 International Conference on Advanced Logistics and Transport, pages 500-505, New York, USA, 2013.

[99] E. Hamrock, K. Paige, J. Parks, J. Scheulen, and S. Levin. Discrete event simulation for healthcare organizations: A tool for decision making. Journal of Healthcare Management, 58(2):110-124, 2013.

[100] E. Hans, G. Wullink, M. van Houdenhoven, and G. Kazemier. Robust surgery loading. European Journal of Operational Research, 185(3):1038-1050, 2008.

[101] E. W. Hans and T. Nieberg. Operating room manager game. INFORMS Transactions on Education, 8(1):25-36, 2007.

[102] E. W. Hans, M. van Houdenhoven, and P. J. Hulshof. A framework for healthcare planning and control. In R. Hall, editor, Handbook of Healthcare System Scheduling, volume 168 of International Series in Operations Research 83 Management Science, pages 303-320. Springer, Berlin, Germany, 2012.

[103] J. E. Helm, M. Lapp, and B. D. See. Characterizing an effective hospital admissions scheduling and control management system: A genetic algorithm approach. In B. Johansson, S. Jain, J. MontoyaTorres, J. Hugan, and E. Yucesan, editors, Proceedings of the Winter Simulation Conference, pages 2387-2398, Baltimore, USA, 2010. 
[104] J. E. Helm, S. AhmadBeygi, and M. P. Van Oyen. Design and analysis of hospital admission control for operational effectiveness. Production and Operations Management, 20(3):359-374, 2011.

[105] S. G. Henderson, J. J. Cochran, L. A. Cox, P. Keskinocak, J. P. Kharoufeh, and J. C. Smith. Operations Research Tools for Addressing Current Challenges in Emergency Medical Services. John Wiley \& Sons, Inc., 2010.

[106] M. Heydari and A. Soudi. Predictive/reactive planning and scheduling of a surgical suite with emergency patient arrival. Journal of Medical Systems, 40(1):9, 2016.

[107] L. B. Holm and F. A. Dahl. Simulating the influence of a $45 \%$ increase in patient volume on the emergency department of akershus university hospital. In B. Johansson, S. Jain, J. MontoyaTorres, J. Hugan, and E. Yucesan, editors, Proceedings of the Winter Simulation Conference, pages 2455-2461, Baltimore, USA, 2010.

[108] L. B. Holm, F. A. Dahl, and Ieee. Simulating the effect of physician triage in the emergency department of akershus university hospital. In Proceedings of the Winter Simulation Conference, pages 1847-1856, Austin, USA, 2009.

[109] L. B. Holm, H. Luras, and F. A. Dahl. Improving hospital bed utilisation through simulation and optimisation with application to a $40 \%$ increase in patient volume in a norwegian general hospital. International Journal of Medical Informatics, 82 (2):80-89, 2013.

[110] N. R. Hoot and D. Aronsky. Systematic review of emergency department crowding: Causes, effects, and solutions. Annals of Emergency Medicine, 52(2):126-136, 2008.

[111] J. F. Huang, B. Carmeli, and A. Mandelbaum. Control of patient flow in emergency departments, or multiclass queues with deadlines and feedback. Operations Research, 63(4):892-908, 2015.

[112] Y. L. Huang, P. Zuniga, and J. Marcak. A cost-effective urgent care policy to improve patient access in a dynamic scheduled clinic setting. Journal of the Operational Research Society, 65(5):763-776, 2014.

[113] W. T. Huh, N. Liu, and V. A. Truong. Multiresource allocation scheduling in dynamic environments. Manufacturing \& Service Operations Management, 15 (2):280-291, 2013.

[114] P. J. H. Hulshof, N. Kortbeek, R. J. Boucherie, E. W. Hans, and P. J. M. Bakker. Taxonomic classification of planning decisions in health care: a structured review of the state of the art in OR/MS. Health Systems, 1(2):129-175, 2012.

[115] P. J. Hulshof, R. J. Boucherie, E. W. Hans, and J. L. Hurink. Tactical resource allocation and elective patient admission planning in care processes. Health care management science, 16(2):152-166, 2013. 
[116] A. Ingolfsson. EMS planning and management. In G. S. Zaric, editor, Operations Research and Health Care Policy, pages 105-128. Springer New York, New York, NY, 2013.

[117] Institute for Operations Research and the Management Sciences (INFORMS). What is operations research. Accessed January 2017 (available at: https://www.informs.org/About-INFORMS/What-is-Operations-Research).

[118] K. Ismail, W. Abo-Hamad, and A. Arisha. Integrating balanced scorecard and simulation modeling to improve emergency department performance in irish hospitals. In B. Johansson, S. Jain, J. MontoyaTorres, J. Hugan, and E. Yucesan, editors, Proceedings of the Winter Simulation Conference, pages 2340-2351, Baltimore, USA, 2010.

[119] T. Ivanova, L. Malone, and M. Mollaghasemi. Comparison of a two-stage groupscreening design to a standard 2k-p design for a whole-line semiconductor manufacturing simulation model. In Proceedings of the Winter Simulation Conference, pages 640-646, Phoenix, USA, 1999.

[120] N. Izady. Appointment capacity planning in specialty clinics: A queueing approach. Operations Research, 63(4):916-930, 2015.

[121] N. Izady and D. Worthington. Setting staffing requirements for time dependent queueing networks: The case of accident and emergency departments. European Journal of Operational Research, 219(3):531-540, 2012.

[122] B. Jerbi and H. Kamoun. Using simulation and goal programming to reschedule emergency department doctors' shifts: case of a tunisian hospital. Journal of Simulation, 3(4):211-219, 2009.

[123] L. X. Jiang and R. E. Giachetti. A queueing network model to analyze the impact of parallelization of care on patient cycle time. Health Care Management Science, 11(3):248-261, 2008.

[124] J. Jihene, A. El Mhamedi, and H. Chabchoub. Simulationmodel of emergency department. In 2007 International Conference on Service Systems and Service Management, pages 402-406, New York, 2007.

[125] P. Jittamai and T. Kangwansura. A hospital admission planning model for emergency and elective patients under stochastic resource requirements and no-shows. 2011 Ieee International Conference on Industrial Engineering and Engineering Management (Ieem), pages 166-170, 2011.

[126] D. Jolly, I. Nouaouri, and J. C. Nicolas. Operating room scheduling under unexpected events: the case of a disaster. Journal of Applied Operational Research, 3 (3):163-176, 2011.

[127] S. A. Jones, M. P. Joy, and J. Pearson. Forecasting demand of emergency care. Health Care Management Science, 5(4):297-305, 2002.

[128] J. B. Jun, S. H. Jacobson, and J. R. Swisher. Application of discrete-event simulation in health care clinics: A survey. Journal of the Operational Research Society, 50(2):109-123, 1999. 
[129] C. J. Jurishica. Emergency department simulations: medicine for building effective models. In Proceedings of the Winter Simulation Conference, pages 26742680, Orlando, USA, 2005.

[130] F. Kadri, F. Harrou, S. Chaabane, and C. Tahon. Time series modelling and forecasting of emergency department overcrowding. Journal of Medical Systems, 38(9):20, 2014.

[131] A. Kanagarajah, D. Parker, and H. Xu. Health care supply networks in tightly and loosely coupled structures: exploration using agent-based modelling. International Journal of Systems Science, 41(3):261-270, 2010.

[132] A. Kaushal, Y. C. Zhao, Q. J. Peng, T. Strome, E. Weldon, M. Zhang, and A. Chochinov. Evaluation of fast track strategies using agent-based simulation modeling to reduce waiting time in a hospital emergency department. SocioEconomic Planning Sciences, 50:18-31, 2015.

[133] P. Kazemian, Y. Dong, T. R. Rohleder, J. E. Helm, and M. P. Van Oyen. An ip-based healthcare provider shift design approach to minimize patient handoffs. Health Care Management Science, 17(1):1-14, 2014.

[134] W. D. Kelton. Experimental design for simulation. In Proceedings of the Winter Simulation Conference, pages 32-38 vol.1, Orlando, USA, 2000.

[135] W. D. Kelton and A. M. Law. Simulation modeling and analysis. McGraw Hill, Boston, 3rd edition, 2000.

[136] M. Khadem, H. A. Bashir, Y. Al-Lawati, and F. Al-Azri. Evaluating the layout of the emergency department of a public hospital using computer simulation modeling: A case study. In Ieem: 2008 International Conference on Industrial Engineering and Engineering Management, pages 1709-1713, New York, 2008.

[137] S. Kim and S. Kim. Differentiated waiting time management according to patient class in an emergency care center using an open jackson network integrated with pooling and prioritizing. Annals of Operations Research, 230(1):35-55, 2015.

[138] J. P. C. Kleijnen. Design of experiments: Overview. In Proceedings of the Winter Simulation Conference, pages 479-488, Miami, USA, Dec 2008.

[139] A. J. Kleywegt, A. Shapiro, and T. H. de Mello. The sample average approximation method for stochastic discrete optimization. SIAM Journal on Optimization, $12(2): 479-502,2002$.

[140] P. M. Koeleman and G. M. Koole. Appointment scheduling using optimisation via simulation. In Proceedings of the Winter Simulation Conference, page 22, Berlin, Germany, 2012.

[141] P. M. Koeleman and G. M. Koole. Optimal outpatient appointment scheduling with emergency arrivals and general service times. IIE Transactions on Healthcare Systems Engineering, 2(1):14-30, 2012. 
[142] E. M. W. Kolb, T. Lee, and J. Peck. Effect of coupling between emergency department and inpatient unit on the overcrowding in emergency department. In Proceedings of the Winter Simulation Conference, pages 1565-1572, Washington D.C., USA, 2007.

[143] R. Kolisch and S. Sickinger. Providing radiology health care services to stochastic demand of different customer classes. Or Spectrum, 30(2):375-395, 2008.

[144] A. Komashie and A. Mousavi. Modeling emergency departments using discrete event simulation techniques. In Proceedings of the Winter Simulation Conference, pages 2681-2685, Orlando, USA, Dec 2005.

[145] R. Konrad, K. DeSotto, A. Grocela, P. McAuley, J. Wang, J. Lyons, and M. Bruin. Modeling the impact of changing patient flow processes in an emergency department: Insights from a computer simulation study. Operations Research for Health Care, 2(4):66-74, 2013.

[146] N. Kortbeek, M. E. Zonderland, A. Braaksma, I. M. Vliegen, R. J. Boucherie, N. Litvak, and E. W. Hans. Designing cyclic appointment schedules for outpatient clinics with scheduled and unscheduled patient arrivals. Performance Evaluation, $80: 5-26,2014$.

[147] N. Kortbeek, A. Braaksma, F. H. Smeenk, P. J. Bakker, and R. J. Boucherie. Integral resource capacity planning for inpatient care services based on bed census predictions by hour. Journal of the Operational Research Society, 66(7):10611076, 2015.

[148] H. Kulatunga, W. J. Knottenbelt, and V. Kadirkamanathan. Adaptive Planning of Staffing Levels in Health Care Organisations, volume 27 of Lecture Notes of the Institute for Computer Sciences Social Informatics and Telecommunications Engineering, pages 88-95. Springer-Verlag Berlin, Berlin, 2010.

[149] A. Kumar, R. J. Jiao, and S. J. Shim. Predicting bed requirement for a hospital using regression models. In 2008 IEEE International Conference on Industrial Engineering and Engineering Management, pages 665-669, Singapore, 2008.

[150] S. Kumar. Modeling hospital surgical delivery process design using system simulation: Optimizing patient flow and bed capacity as an illustration. Technology and Health Care, 19(1):1-20, 2011.

[151] S. Kumar. Modeling patient flow operation of a us urban county hospital. Technology and Health Care, 19(4):247-260, 2011.

[152] Y. H. Kuo, O. Rado, B. Lupia, J. M. Y. Leung, and C. A. Graham. Improving the efficiency of a hospital emergency department: a simulation study with indirectly imputed service-time distributions. Flexible Services and Manufacturing Journal, 28(1-2):120-147, 2016.

[153] M. Lamiri, J. Dreo, and X. L. Xie. Operating room planning with random surgery times. 2007 Ieee International Conference on Automation Science and Engineering, Vols 1-3, pages 495-500, 2007. 
[154] M. Lamiri, X. L. Xie, and S. G. Zhang. Column generation approach to operating theater planning with elective and emergency patients. Iie Transactions, 40(9): 838-852, 2008.

[155] M. Lamiri, X. Xie, A. Dolgui, and F. Grimaud. A stochastic model for operating room planning with elective and emergency demand for surgery. European Journal of Operational Research, 185(3):1026 - 1037, 2008.

[156] M. Lamiri, F. Grimaud, and X. Xie. Optimization methods for a stochastic surgery planning problem. International Journal of Production Economics, 120 (2):400 - 410, 2009.

[157] D. C. Lane, C. Monefeldt, and J. V. Rosenhead. Looking in the wrong place for healthcare improvements: A system dynamics study of an accident and emergency department. Journal of the Operational Research Society, 51(5):518-531, 2000.

[158] M. van der Lans, E. Hans, J. Hurink, G. Wullink, M. van Houdenhoven, and G. Kazemier. Anticipating urgent surgery in operating room departments. Beta working paper vol. 158 (available at: http://repository.tue.nl/), University of Twente, Enschede, 2006.

[159] V. Lattimer, S. Brailsford, J. Turnbull, P. Tarnaras, H. Smith, S. George, K. Gerard, and S. Maslin-Protero. Reviewing emergency care systems i: insights from system dynamics modelling. Emergency Medicine Journal, 21(6):685-691, 2004.

[160] A. M. Law. Simulation modeling and analysis. McGraw-Hill, New York, 4th edition, 2007.

[161] E. K. Lee, H. Y. Atallah, M. D. Wright, E. T. Post, C. Thomas, D. T. Wu, and L. L. Haley. Transforming hospital emergency department workflow and patient care. Interfaces, 45(1):58-82, 2015.

[162] A. G. Leeftink and E. W. Hans. Case mix classification and benchmark set for surgery scheduling. working paper, University of Twente, Enschede, 2016.

[163] S. Levin, J. Han, D. Aronsky, C. Zhou, N. Hoot, L. Kelly, and D. France. Stranded on emergency isle: Modeling competition for cardiac services using survival analysis. In 2007 Ieee International Conference on Industrial Engineering and Engineering Management, pages 1772-1776. 2007.

[164] D. Li and K. D. Glazebrook. A bayesian approach to the triage problem with imperfect classification. European Journal of Operational Research, 215(1):169$180,2011$.

[165] X. Li, Z. Zhao, X. Zhu, and T. Wyatt. Covering models and optimization techniques for emergency response facility location and planning: a review. Mathematical Methods of Operations Research, 74(3):281-310, 2011.

[166] D. Lin, J. Patrick, and F. Labeau. Estimating the waiting time of multi-priority emergency patients with downstream blocking. Health Care Management Science, 17(1):88-99, 2014. 
[167] R. Litjens and R. Boucherie. Performance analysis of fair channel sharing policies in an integrated cellular voice/data network. Telecommunication systems, 19(2): 147-186, 2002.

[168] E. Litvak and M. C. Long. Cost and quality under managed care: Irreconcilable differences. American Journal of Managed Care, 6(3):305-12, 2000.

[169] N. Litvak, M. van Rijsbergen, R. J. Boucherie, and M. van Houdenhoven. Managing the overflow of intensive care patients. European Journal of Operational Research, 185(3):998-1010, 2008.

[170] M. E. Lübbecke and J. Desrosiers. Selected topics in column generation. Operations Research, 53(6):1007-1023, 2005.

[171] S. Lucidi, M. Maurici, L. Paulon, F. Rinaldi, and M. Roma. A derivative-free approach for a simulation-based optimization problem in healthcare. Optimization Letters, 10(2):219-235, 2016.

[172] L. Luo, J. Ji, H. Tang, and Q. Ren. Optimal allocation of CT scan capacity among stochastic demand of different customer classes. Service Systems and Service Management (ICSSSM), 2014 11th International Conference on, pages 1-6, 2014.

[173] R. Luscombe and E. Kozan. Dynamic resource allocation to improve emergency department efficiency in real time. European Journal of Operational Research, 255(2):593-603, 2016.

[174] O. H. C. Magdalene, Z. Dali, P. T. Beng, and C. P. Yoke. Uncovering effective process improvement strategies in an emergency department using discrete event simulation. Health Systems, 3(2):93-104, 2014.

[175] A. Mandelbaum, P. Momcilovic, and Y. Tseytlin. On fair routing from emergency departments to hospital wards: Qed queues with heterogeneous servers. Management Science, 58(7):1273-1291, 2012.

[176] Y. N. Marmor, B. Golany, S. Israelit, and A. Mandelbaum. Designing patient flow in emergency departments. IIE Transactions on Healthcare Systems Engineering, 2(4):233-247, 2012.

[177] C. A. Mauro. On the performance of two-stage group screening experiments. Technometrics, 26(3):255-264, 1984.

[178] J. H. May, D. P. Strum, and L. G. Vargas. Fitting the lognormal distribution to surgical procedure times*. Decision Sciences, 31(1):129-148, 2000.

[179] L. Mayhew and D. Smith. Using queuing theory to analyse the government's 4-h completion time target in accident and emergency departments. Health Care Management Science, 11(1):11-21, 2008.

[180] A. Mazier, X. Xie, and M. Sarazin. Scheduling inpatient admission under high demand of emergency patients. Automation Science and Engineering (CASE), 2010 IEEE Conference on, pages 792-797, 2010. 
[181] M. Mes and M. Bruens. A generalized simulation model of an integrated emergency post. In Proceedings of the Winter Simulation Conference, pages 1-11, Berlin, Germany, 2012.

[182] B. Mielczarek and J. Uziałko-Mydlikowska. Application of computer simulation modeling in the health care sector: a survey. Simulation, 88(2):197-216, 2012.

[183] M. Miller, D. Ferrin, M. Ashby, T. Flynn, N. Shahi, and Ieee. Merging six emergency departments into one: A simulation approach. In Proceedings of the Winter Simulation Conference, pages 1553-1557, Washington D.C., USA, 2007.

[184] D. Min and Y. Yih. Managing a patient waiting list with time-dependent priority and adverse events. RAIRO-Operations Research, 48(1):53-74, 2014.

[185] D. Min and Y. Yih. Scheduling elective surgery under uncertainty and downstream capacity constraints. European Journal of Operational Research, 206(3): $642-652,2010$.

[186] D. Min and Y. Yih. An elective surgery scheduling problem considering patient priority. Computers \& Operations Research, 37(6):1091 - 1099, 2010.

[187] D. C. Montgomery. Design and analysis of experiments. John Wiley \& Sons, US, 7 th edition, 2008.

[188] G. Mould, J. Bowers, C. Dewar, and E. McGugan. Assessing the impact of systems modeling in the redesign of an emergency department. Health Systems, $2(1): 3-10,2013$.

[189] M. Murray, M. Bullard, and E. Grafstein. Revisions to the canadian emergency department triage and acuity scale implementation guidelines. Canadian Journal of Emergency Medicine, 6, 2004.

[190] Nederlandse Vereniging van Ziekenhuizen (NVZ). Acute zorg - visiedocument [in Dutch]. Published by the NVZ (available at: https://www.nvz-ziekenhuizen.nl/), 2013.

[191] A. L. Nielsen, H. Hilwig, N. Kissoon, and S. Teelucksingh. Discrete event simulation as a tool in optimization of a professional complex adaptive system, volume 136 of Studies in Health Technology and Informatics, pages 247-252. IOS Press, Amsterdam, 2008.

[192] I. Nouaouri, J. C. Nicolas, and D. Jolly. Scheduling of stabilization surgical cares in case of a disaster. In 2009 Ieee International Conference on Industrial Engineering and Engineering Management, pages 1974-1978. 2009.

[193] J. P. Oddoye, M. A. Yaghoobi, M. Tamiz, D. F. Jones, and P. Schmidt. A multiobjective model to determine efficient resource levels in a medical assessment unit. Journal of the Operational Research Society, 58(12):1563-1573, 2007.

[194] J. P. Oddoye, D. F. Jones, M. Tamiz, and P. Schmidt. Combining simulation and goal programming for healthcare planning in a medical assessment unit. European Journal of Operational Research, 193(1):250-261, 2009. 
[195] J. M. van Oostrum, M. Van Houdenhoven, M. M. Vrielink, J. Klein, E. W. Hans, M. Klimek, G. Wullink, E. W. Steyerberg, and G. Kazemier. A simulation model for determining the optimal size of emergency teams on call in the operating room at night. Anesthesia and Analgesia, 107(5):1655-62, 2008.

[196] J. M. van Oostrum, M. Van Houdenhoven, J. L. Hurink, E. W. Hans, G. Wullink, and G. Kazemier. A master surgical scheduling approach for cyclic scheduling in operating room departments. OR spectrum, 30(2):355-374, 2008.

[197] J. Patrick and M. L. Puterman. Improving resource utilization for diagnostic services through flexible inpatient scheduling: A method for improving resource utilization. Journal of the Operational Research Society, 58(2):235-245, 2007.

[198] J. Patrick, M. L. Puterman, and M. Queyranne. Dynamic multipriority patient scheduling for a diagnostic resource. Operations Research, 56(6):1507-1525, 2008.

[199] J. A. Paul and L. MacDonald. Determination of number of dedicated or's and supporting pricing mechanisms for emergent surgeries. Journal of the Operational Research Society, 64(6):912-924, 2013.

[200] S. A. Paul, M. C. Reddy, and C. J. DeFlitch. A systematic review of simulation studies investigating emergency department overcrowding. Simulation, 86(8-9): $559-571,2010$.

[201] M. J. Persson and J. A. Persson. Analysing management policies for operating room planning using simulation. Health Care Management Science, 13(2):182191, 2010.

[202] D. Petrovic, M. Morshed, and S. Petrovic. Multi-objective genetic algorithms for scheduling of radiotherapy treatments for categorised cancer patients. Expert Systems with Applications, 38(6):6994-7002, 2011.

[203] D. Pham and A. Klinkert. Surgical case scheduling as a generalized job shop scheduling problem. European Journal of Operational Research, 185(3):1011$1025,2008$.

[204] J. Pirolo, A. Ray, M. Gadzinski, M. Manese, B. Garvert, G. Scoville, H. Walpole, B. Amland, R. Boos, I. Mamminga, J. Brown, K. Donlon, and Ieee. Utilization of discrete event simulation in the prospective determination of optimal cardiovascular lab processes. In Proceedings of the Winter Simulation Conference, pages 1867-1877, Austin, USA, 2009.

[205] M. Pitt, T. Monks, S. Crowe, and C. Vasilakis. Systems modelling and simulation in health service design, delivery and decision making. BMJ Quality \& Safety, 25 (1):38-45, 2016.

[206] S. S. Qiu, R. B. Chinnam, A. Murat, B. Batarse, H. Neemuchwala, and W. Jordan. A cost sensitive inpatient bed reservation approach to reduce emergency department boarding times. Health Care Management Science, 18(1):67-85, 2015.

[207] S. Rachuba and B. Werners. A robust approach for scheduling in hospitals using multiple objectives. Journal of the Operational Research Society, 65(4):546-556, 2014 . 
[208] F. Rasheed, Y. H. Lee, S. H. Kim, and I. C. Park. Development of emergency department load relief area-gauging benefits in empirical terms. Simulation in Healthcare-Journal of the Society for Simulation in Healthcare, 7(6):343-352, 2012 .

[209] W. Rashwan, W. Abo-Hamad, and A. Arisha. A system dynamics view of the acute bed blockage problem in the irish healthcare system. European Journal of Operational Research, 247(1):276-293, 2015.

[210] F. Rico, E. Salari, G. Centeno, and Ieee. Emergency departments nurse allocation to face a pandemic influenza outbreak. In Proceedings of the Winter Simulation Conference, pages 1271-1277, Washington D.C., USA, 2007.

[211] S. Saghafian, W. J. Hopp, M. P. Van Oyen, J. S. Desmond, and S. L. Kronick. Patient streaming as a mechanism for improving responsiveness in emergency departments. Operations Research, 60(5):1080-1097, 2012.

[212] S. Saghafian, W. J. Hopp, M. P. Van Oyen, J. S. Desmond, and S. L. Kronick. Complexity-augmented triage: A tool for improving patient safety and operational efficiency. Manufacturing \& Service Operations Management, 16(3): 329-345, 2014.

[213] R. Sampath and H. Darabi. Control reconfiguration of hospital operations with dynamic resources. In Proceedings of the Sixth Iasted International Conference on Intelligent Systems and Control, pages 76-81, Calgary, 2004.

[214] B. E. Sandbaek, B. I. Helgheim, O. I. Larsen, and S. Fasting. Impact of changed management policies on operating room efficiency. BMC Health Services Research, 14:224, 2014.

[215] J. Segrist, W. R. Reinus, A. Enyan, P. Flanagan, B. Pim, and D. S. Sallee. A proposed scheduling model to improve use of computed tomography facilities. Journal of Medical Systems, 24(2):61-76, 2000.

[216] S. Sengupta, M. Deneweth, and R. Van Til. A better approach to modeling emergency care service. In S. Jain, R. Creasey, and J. Himmelspach, editors, Proceedings of the Winter Simulation Conference, pages 1202-1210, Phoenix, USA, 2011.

[217] P. Y. Shi, M. C. Chou, J. G. Dai, D. Ding, and J. Sim. Models and insights for hospital inpatient operations: Time-dependent ed boarding time. Management Science, 62(1):1-28, 2016.

[218] S. Sickinger and R. Kolisch. The performance of a generalized bailey-welch rule for outpatient appointment scheduling under inpatient and emergency demand. Health Care Management Science, 12(4):408-419, 2009.

[219] D. Sinreich and Y. Marmor. Emergency department operations: The basis for developing a simulation tool. Iie Transactions, 37(3):233-245, 2005.

[220] D. Sinreich, O. Jabali, and N. P. Dellaert. Reducing emergency department waiting times by adjusting work shifts considering patient visits to multiple care providers. Iie Transactions, 44(3):163-180, 2012. 
[221] O. Sinreich, D.; Jabali. Staggered work shifts: a way to downsize and restructure an emergency department workforce yet maintain current operational performance. Health Care Management Science, 10(3):293-308, 2007.

[222] P. S. Stepaniak, C. Heij, G. H. H. Mannaerts, M. de Quelerij, and G. de Vries. Modeling procedure and surgical times for current procedural terminologyanesthesia-surgeon combinations and evaluation in terms of case-duration prediction and operating room efficiency: A multicenter study. Anesthesia and Analgesia, 109(4):1232-1245, October 2009.

[223] Tableau Software. Business intelligence software. tableau desktop (version 9.2) [computer software]. (available at: http://www.tableau.com/products/desktop), 2016.

[224] S. Takakuwa and H. Shiozaki. Functional analysis for operating emergency department of a general hospital. In Proceedings of the Winter Simulation Conference, pages 2003-2011, Washington D.C., USA, 2004.

[225] J. S. Tancrez, B. Roland, J. P. Cordier, and F. Riane. How stochasticity and emergencies disrupt the surgical schedule. Studies in Computational Intelligence, 189:221-239, 2009.

[226] J. S. Tancrez, B. Roland, J. P. Cordier, and F. Riane. Assessing the impact of stochasticity for operating theater sizing. Decision Support Systems, 55(2): 616-628, 2013.

[227] E. Tanfani and A. Testi. A pre-assignment heuristic algorithm for the master surgical schedule problem (mssp). Annals of Operations Research, 178(1):105119, 2010.

[228] J. F. Tang and Y. Wang. An adjustable robust optimisation method for elective and emergency surgery capacity allocation with demand uncertainty. International Journal of Production Research, 53(24):7317-7328, 2015.

[229] J. F. Tang, C. J. Yan, and P. P. Cao. Appointment scheduling algorithm considering routine and urgent patients. Expert Systems with Applications, 41(10): 4529-4541, 2014.

[230] A. Testi and E. Tànfani. Tactical and operational decisions for operating room planning: Efficiency and welfare implications. Health Care Management Science, 12(4):363-373, 2009.

[231] S. Thompson, M. Nunez, R. Garfinkel, and M. D. Dean. Efficient short-term allocation and reallocation of patients to floors of a hospital during demand surges. Operations Research, 57(2):261-273, 2009.

[232] A. Tirdad, W. K. Grassmann, and J. Tavakoli. Optimal policies of $\mathrm{m}(\mathrm{t}) / \mathrm{m} / \mathrm{c} / \mathrm{c}$ queues with two different levels of servers. European Journal of Operational Research, 249(3):1124-1130, 2016. 
[233] L. Trocine and L. C. Malone. Experimental design and analysis: An overview of newer, advanced screening methods for the initial phase in an experimental design. In Proceedings of the Winter Simulation Conference, pages 169-178, Arlington, USA, 2001.

[234] V. A. Truong. Optimal advance scheduling. Management Science, 61(7):1584$1597,2015$.

[235] Twijnstra Gudde. De brede betekenis van acute zorg [in Dutch]. Factsheet published by Nederlandse Vereniging van Ziekenhuizen (available at: https://www.nvz-ziekenhuizen.nl/), 2013.

[236] D. C. Tyler, C. A. Pasquariello, and C. H. Chen. Determining optimum operating room utilization. Anesthesia and Analgesia, 96(4):1114-1121, 2003.

[237] M. Utley, S. Gallivan, K. Davis, P. Daniel, P. Reeves, and J. Worrall. Estimating bed requirements for an intermediate care facility. European Journal of Operational Research, 150(1):92-100, 2003.

[238] C. Van Riet and E. Demeulemeester. Trade-offs in operating room planning for electives and emergencies: a review. Operations Research for Health Care, 7: $52-69,2015$.

[239] E. van Veen-Berkx, S. G. Elkhuizen, B. Kuijper, and G. Kazemier. Dedicated operating room for emergency surgery generates more utilization, less overtime, and less cancellations. American Journal of Surgery, 211(1):122-128, 2016.

[240] I. Vermeulen, S. Bohte, S. Elkhuizen, J. Lameris, P. Bakker, and J. La Poutre. Adaptive optimization of hospital resource calendars. Lecture Notes in Computer Science, 4594:305-315, 2007.

[241] J. M. Vissers, I. J. Adan, and N. P. Dellaert. Developing a platform for comparison of hospital admission systems: An illustration. European Journal of Operational Research, 180(3):1290 - 1301, 2007.

[242] S. Walczak, W. E. Pofahl, and R. J. Scorpio. A decision support tool for allocating hospital bed resources and determining required acuity of care. Decision Support Systems, 34(4):445-456, 2003.

[243] J. W. Wang, J. S. Li, and P. K. Howard. A system model of work flow in the patient room of hospital emergency department. Health Care Management Science, 16(4):341-351, 2013.

[244] T. Wang, A. Guinet, A. Belaidi, and B. Besombes. Modelling and simulation of emergency services with aris and arena. case study: the emergency department of saint joseph and saint luc hospital. Production Planning \& Control, 20(6): 484-495, 2009.

[245] T. Wang, A. Guinet, and B. Besombes. A sizing tool for allocation planning of hospital bed resources. In S. McClean, P. Millard, E. El-Darzi, and C. Nugent, editors, Intelligent Patient Management, pages 113-125. Springer Berlin Heidelberg, Berlin, 2009. 
[246] Y. Wang, W. L. Hare, L. Vertesi, and A. R. Rutherford. Using simulation to model and optimize acute care access in relation to hospital bed count and bed distribution. Journal of Simulation, 5(2):101-110, 2011.

[247] Y. Wang, J. F. Tang, and R. Y. K. Fung. A column-generation-based heuristic algorithm for solving operating theater planning problem under stochastic demand and surgery cancellation risk. International Journal of Production Economics, 158:28-36, 2014.

[248] J. Welch and N. J. Bailey. Appointment systems in hospital outpatient departments. The Lancet, 259(6718):1105-1108, 1952.

[249] S. J. Weng, T. R. Wu, G. Mackulak, and J. Fowler. Distributed resource allocation for healthcare systems. In Proceedings of 2008 Ieee International Conference on Service Operations and Logistics, and Informatics, pages 1078-1083. 2008.

[250] S. J. Weng, B. C. Cheng, S. T. Kwong, L. M. Wang, and C. Y. Chang. Simulation optimization for emergency department resources allocation. In S. Jain, R. Creasey, and J. Himmelspach, editors, Proceedings of the Winter Simulation Conference, pages 1231-1238, Phoenix, USA, 2011.

[251] S. J. Weng, B. S. Tsai, L. M. Wang, C. Y. Chang, and D. Gotcher. Using simulation and data envelopment analysis in optimal healthcare efficiency allocations. In S. Jain, R. Creasey, and J. Himmelspach, editors, Proceedings of the Winter Simulation Conference, pages 1295-1305, Phoenix, USA, 2011.

[252] J. J. Wixted, M. Reed, M. S. Eskander, B. Millar, R. C. Anderson, K. Bagchi, S. Kaur, P. Franklin, and W. Leclair. The effect of an orthopedic trauma room on after-hours surgery at a level one trauma center. Journal of Orthopaedic Trauma, 22(4):234-236, April 2008.

[253] G. Wullink, M. Van Houdenhoven, E. W. Hans, J. M. van Oostrum, M. van der Lans, and G. Kazemier. Closing emergency operating rooms improves efficiency. Journal of Medical Systems, 31(6):543-546, 2007.

[254] I. van der Wulp, M. E. van Baar, and A. J. Schrijvers. Reliability and validity of the manchester triage system in a general emergency department patient population in the netherlands: results of a simulation study. Emergency Medicine Journal, 25, 2008.

[255] Y. S. Xiang and J. Zhuang. A medical resource allocation model for serving emergency victims with deteriorating health conditions. Annals of Operations Research, 236(1):177-196, 2016.

[256] M. Xu, T. C. Wong, K. S. Chin, S. Y. Wong, and K. L. Tsui. Modeling patient visits to accident and emergency department in hong kong. 2011 Ieee International Conference on Industrial Engineering and Engineering Management (Ieem), pages 1730-1734, 2011.

[257] R. Yaesoubi, S. D. Roberts, and R. W. Klein. Modification of cheng's method: An alternative factor screening method for stochastic simulation models. In Proceedings of the Winter Simulation Conference, pages 1034-1047, Baltimore, USA, 2010. 
[258] N. Yankovic and L. V. Green. Identifying good nursing levels: A queuing approach. Operations Research, 59(4):942-955, 2011.

[259] J. Y. Yeh and W. S. Lin. Using simulation technique and genetic algorithm to improve the quality care of a hospital emergency department. Expert Systems with Applications, 32(4):1073-1083, 2007.

[260] G. B. Yom-Tov and A. Mandelbaum. Erlang-r: A time-varying queue with reentrant customers, in support of healthcare staffing. Manufacturing $\&$ Service Operations Management, 16(2):283-299, 2014.

[261] X. Zeng, Zhen; Ma, Y. Hu, J. Li, and D. Bryant. A simulation study to improve quality of care in the emergency department of a community hospital. Journal of Emergency Nursing, 38(4):322-328, 2012.

[262] B. Zhang, P. Murali, M. M. Dessouky, and D. Belson. A mixed integer programming approach for allocating operating room capacity. Journal of the Operational Research Society, 60(5):663-673, 2009.

[263] M. E. Zonderland, R. J. Boucherie, N. Litvak, and C. Vleggeert-Lankamp. Planning and scheduling of semi-urgent surgeries. Health Care Management Science, 13(3):256-267, 2010. 



\section{Acronyms}

ATS Australasian Triage Scale

BIM Break In Moments

CG Column Generation

CHOIR Center for Healthcare Operations Improvement and Research

CTAS Canadian Triage and Acuity Scale

CV Co-efficient of Variation

DES Discrete Event Simulation

DTC Diagnostic Treatment Combination

ED Emergency Department

EMS Emergency Medical Services

ESI Emergency Severity Index

EVP Expected Value Problem

FCFS First-Come First-Serve

GP General Practitioner

GPP General Practitioners Post

Haga HagaZiekenhuis

ICU Intensive Care Unit

IEP Integrated Emergency Post

IFFD Iterated Fractional Factorial Design

ILP Integer Linear Program

LOS Length Of Stay

MAU Medical Assessment Unit

MAUT Multi Attribute Utility Theory

MDP Markov Decision Process

MILP Mixed Integer Linear Program

MRP Markov Reward Process

MSS Master Surgery Schedule

MTS Manchester Triage Scale

OR Operating Room

ORDS Operating Room Day Schedules

OT Operating Theater

PACU Post Anesthetic Care Unit

SAA Sample Average Approximation

SSD Supersaturated Designs

STS Surgery Type Schedule 



\section{Summary}

One of the challenges of hospitals is to take into account the many uncertainties that arise during the delivery of care. This is especially prevalent when providing care for non-elective patients who require treatment with some urgency. As urgency increases, the stakes involved increase for both patients and healthcare providers. Non-elective care is prevalent throughout hospitals. The question then is how to efficiently organize healthcare processes while remaining able to properly adapt to the specific needs and characteristics of non-elective patient demand. In this thesis we address a number of challenges related to the provision of care for non-elective patients. The problems studied in this dissertation originate from hospitals in the Netherlands, and address planning problems encountered at various hospital departments where non-elective patients are treated.

Chapter 2 reviews non-elective care planning and scheduling literature within and across hospital departments, and structurally categorizes the literature according to departmental focus, hierarchical planning level, and urgency classification. Furthermore, it provides an overview of the non-elective healthcare planning and control problems that are considered, and outlines what methods are used to address these problems. Finally, Chapter 2 identifies some interesting future research opportunities.

Chapter 3 uses a systematic heuristic approach and computer simulation to evaluate and find the optimal process design of an integrated emergency post, where an emergency department and general practitioners post have merged. By evaluating resource and staff allocations, as well as process changes, various designs are compared. In the design and evaluation of the interventions, patient preferences are taken into account, and the approach and computer simulation model are set up so it can readily be applied to other healthcare providers. Results show that the merging of ED and GPP offer numerous improvement possibilities through cooperation and combined allocation of resources. The selected interventions show a significant improvement in length of stay, with roster alternatives having the greatest effect on the GPP, and process interventions on the ED. In addition, the pooling of resources, such as staff, shows that both the GPP and ED can benefit, while the overall staffing costs remain virtually unchanged. Finally, the interventions have no negative effects on any patient urgency category.

In Chapter 4 we propose a heuristic solution methodology to optimize (CT diagnostic) appointment schedules for elective patients in a radiology department that also offers diagnostics to semi-urgent patients who may arrive from the ED or hospital wards. Using this approach we take into account the arrival of non- 
elective patients and find that the appointment schedule may be changed such that waiting time for both elective and non-elective patients is reduced. This is achieved by creating an appointment schedule that offers appointments to elective patients at times during the day when fewer non-elective patients arrive. Given the large number of possible appointment schedules, as well as stochastic nature of non-elective patient arrivals we combine our heuristic approach with Markov process for smaller cases, and use computer simulation for larger instance sizes. Applying this approach to HagaZiekenhuis data we find that besides the waiting time reductions for patients, overtime is also reduced, and the diagnostics utilization is spread more evenly across the day.

In chapter 5 we evaluate various policies for operating theaters to allocate operating room capacity to emergency patients using a discrete event simulation model. These policies are (1) to reserve dedicated ORs for emergency patients, (2) to let emergency patients interrupt and break into the elective surgery program, or (3) a combination of the two aforementioned policies. We identify and incorporate key patient and hospital characteristics that influence these policies such as patient case mix and hospital size, and evaluate the policies' effects on performance indicators including utilization, elective and emergency waiting times, and overtime. The simulation model outcomes are incorporated into an interactive tool to enable quick comparison of alternative policies and settings, and allows hospitals to find a policy configuration best for their case setting. Results show that there is no single policy that performs best in all situations, and we find that there is a trade-off in performance between using a dedicated or break-in policy. Using dedicated emergency ORs leads to lower waiting times for elective patients, but outliers in emergency waiting times are more likely, as emergency patients may wait considerably longer when all emergency ORs are in use. We also find that the main characteristic influencing the effectiveness of policies is the operating theater size. For smaller operating theaters it is more beneficial to use dedicated emergency ORs, as there are otherwise not enough elective ORs to allow for a timely break in into the elective program. The minimum number of ORs lies around eight to nine ORs (in total), before a break-in policy becomes more effective.

In Chapter 6 we use a decomposition approach to create surgery type schedules that incorporate both elective and non-elective patients. The surgery type schedule determines the number, and type, of surgeries that may be planned in an OR, to which at a later point in time surgical cases are assigned. In this approach we first plan the non-elective patients in the surgery type schedule to ensure timely access, and then we use column generation to add elective patients to the schedule such that ORs are best utilized. Results from our case study show that our heuristic approach is able to find surgery type schedules within a reasonable time that reduce non-elective patient waiting times, and are within $5 \%$ of the optimal solution when using multiple OR schedule sizes (e.g., planning half OR days). In addition, by allowing more schedule sizes, considerably fewer ORs are required to schedule all surgery types. Finally, we show that a slight 
increase in the total number of ORs reduces the overtime probability by $20 \%$.

In this dissertation, we consider the planning and control of healthcare processes in hospitals where non-elective patients are prevalent. We focus on solution approaches that account for both elective and non-elective patients. A trade-off is sought between performance indicators of both elective and non-elective patients, as well as a trade-off between efficient and effective care. The work presented in this dissertation may assist hospitals in their decision making, ensuring that both elective and non-elective patients are treated effectively and in a timely manner. Additionally, by specifically incorporating non-elective care in the modeling of processes, outcomes can improve for all patients involved. 



\section{Samenvatting}

Een van de uitdagingen in ziekenhuizen is om rekening te houden met de vele onzekerheden die zich voordoen tijdens de levering van zorg. Dit is veelvoorkomend bij het voorzien van zorg aan patiënten die met enige urgentie een behandeling nodig hebben. Naarmate de urgentie stijgt, nemen de belangen voor zowel patiënten als zorgverleners toe. De vraag is dan hoe zorgprocessen efficiënt georganiseerd kunnen worden die in staat zijn zicht aan te passen aan de specifieke behoeften en karakteristieken van urgente patiënten. In dit proefschrift behandelen we een aantal uitdagingen die gerelateerd zijn aan de levering van zorg aan urgente patiënten. De bestudeerde vraagstukken in dit proefschrift zijn afkomstig vanuit ziekenhuizen in Nederland en behandelen planningsproblemen van verschillende ziekenhuisafdelingen waar urgente patiënten behandeld worden.

Hoofdstuk 2 geeft een overzicht van de literatuur op het gebied van urgente zorgplanning binnen en over ziekenhuisafdelingen heen. In dit hoofdstuk categoriseren we de literatuur op bestudeerde afdelingen, hiërarchisch planningsniveau en urgentie-classificatie. Bovendien biedt het een overzicht van de urgente zorgplanningsvraagstukken en gebruikte methodologieën. Tenslotte identificeert Hoofdstuk 2 interessante toekomstige onderzoeksrichtingen.

Hoofdstuk 3 gebruikt een systematische aanpak en computer simulatie om het optimale procesontwerp van een geïntegreerde spoedpost te vinden en evalueren. Een geïntegreerde spoedpost is een fusie tussen spoedeisende hulp en huisartsenpost. Door verschillende toewijzingen van mens en middelen, evenals proces veranderingen worden interventies geformuleerd en spoedpost ontwerpen vergeleken. In het ontwerp en in de evaluatie van de interventies wordt rekening gehouden met de voorkeuren van patiënten. De resultaten laten zien dat de samenvoeging van spoedeisende hulp en huisartsenpost tal van verbeteringsmogelijkheden biedt door middel van samenwerking en het delen van middelen. De geselecteerde interventies laten een significante verbetering zien voor de verblijfsduur van patiënten, waarbij roosteralternatieven het grootste effect op de huisartsenpost patiënten hebben en proces interventies op de spoedeisende hulp patiënten. Daarnaast blijkt dat met het breed inzetten van personeel beide afdelingen profiteren, zonder toename van personeelskosten.

Hoofdstuk 4 presenteert een heuristiek om het afsprakenschema van electieve patiënten voor CT diagnostiek op een radiologie afdeling te optimaliseren. Deze radiologie afdeling biedt ook diagnostiek aan urgente patiënten die van de spoedeisende hulp of elders uit het ziekenhuis komen. Deze aanpak houdt rekening met de aankomsten van onverwachte urgente patiënten en laat zien dat met aan- 
passingen in het afsprakenschema de wachttijd voor zowel electieve als urgente patiënten afneemt. Deze wachttijd-reductie wordt bereikt door afspraken aan te bieden aan electieve patiënten op tijden van de dag waarin minder urgente patiënten aankomen. Het vinden van het optimale afsprakenschema is complex gegeven het grote aantal mogelijke afsprakenschema's en de stochasticiteit van de aankomsten van urgente patiënten. Daarom modelleren we de afdeling als Markov process voor kleinere instanties en gebruiken we computer simulatie voor grotere instanties. Deze aanpak is toegepast op data van het HagaZiekenhuis en de resultaten laten zien dat naast de wachttijd vermindering voor zowel electieve als urgente patiënten ook overtijd afneemt en de benutting van de diagnostiek apparatuur meer gelijkmatig over de dag is verspreid.

In Hoofdstuk 5 evalueren we verschillende beleidsmaatregelen voor de operatiekamer $(\mathrm{OK})$ afdeling om OK capaciteit in te zetten voor spoedpatiënten met behulp van computersimulatie. Deze maatregelen zijn (1) het inzetten van spoed-OK's, (2) spoedpatiënten laten inbreken in het reguliere programma, en (3) een combinatie van de twee hiervoor genoemde maatregelen. Hoofdstuk 5 identificeert en gebruikt belangrijke ziekenhuis en patiëntkenmerken die invloed hebben op deze beleidsmaatregelen zoals de patiëntenmix en ziekenhuisgrootte, en evalueert de effecten van de beleidsmaatregelen op prestatie-indicatoren zoals OK benutting, wachttijden van electieve en spoedpatiënten en overtijd. De uitkomsten van het computersimulatiemodel zijn samengevoegd in een interactieve tool die het mogelijk maakt snel verschillende beleidsmaatregelen en ziekenhuis situaties te evalueren en vergelijken. Dit stelt zorgmanagers in staat om een beleidsconfiguratie te vinden die het beste toepasbaar is in hun specifieke situatie. De resultaten laten zien dat er geen enkel beleid is dat het beste presteert onder alle omstandigheden en er is een afweging in prestatie-indicatoren tussen een spoed-OK of inbreek beleid. Het gebruik van spoed-OK's leidt tot kortere wachttijden voor electieve patiënten maar spoedpatiënten moeten eventueel lang wachten als alle spoed OK's in gebruik zijn. De resultaten laten ook zien dat de grootste invloed op de effectiviteit van de verschillende beleidsmaatregelen de grootte van de OK afdeling is. Voor kleinere OK afdelingen is het effectiever om spoed-OK's in te zetten, omdat er anders niet genoeg reguliere OK's zijn zodat spoedpatiënten tijdig in kunnen breken in het electieve programma. We zien uit de resultaten dat er rond de 8-9 OK's nodig zijn voordat een inbreek beleid effectiever wordt dan het gebruiken van spoed OK's.

Hoofdstuk 6 introduceert een decompositie aanpak om een operatietype blauwdruk te maken waarin zowel electieve als urgente patiënten worden gepland. Een operatietype blauwdruk bepaalt het aantal en type ingrepen die kunnen worden uitgevoerd in een $\mathrm{OK}$, waarna op een later tijdstip patiënten toegewezen worden. In deze decompositieaanpak worden eerst urgente patiënten aan de blauwdruk toegewezen voor een acceptabele toegangstijd. Vervolgens worden electieve patiënten aan de blauwdruk toegevoegd zodat het benodigde aantal OK's wordt geminimaliseerd. Resultaten van onze casestudy laten zien dat onze aanpak in staat is om operatietype blauwdrukken te maken in een acceptabele 
tijd, die binnen $5 \%$ van de optimale oplossing liggen als er meerdere OK groottes zijn toegestaan (bijvoorbeeld door het toestaan van 'halve' OK dagen). Daarnaast is er het met het toestaan van verschillende OK groottes minder capaciteit nodig om alle operatietypes te plannen. Tenslotte laten we zien dat met een kleine toename in het totaal benodigde aantal OK's de kans op overtijd met $20 \%$ gereduceerd kan worden.

Dit proefschrift behandelt verschillende plannings- en controlevraagstukken van zorgprocessen in ziekenhuizen waar urgente patiënten in voorkomen. We richten ons hierin op oplossingsmethoden die rekening houden met zowel electieve als urgente patiënten en de afwegingen tussen prestatie-indicatoren van electieve en urgente patiënten. Het onderzoek in dit proefschrift is gebaseerd op praktijkcasussen en kan ziekenhuizen ondersteunen in het plannen van zorgprocessen om ervoor te zorgen dat patiënten effectief en tijdig behandeld worden. Daarnaast kunnen door het rekening houden met urgente patiënten in het modelleren van processen de uitkomsten voor alle patiënten verbeteren. 



\section{About the author}

Nardo Jonathan Borgman was born in Hoogeveen, the Netherlands on December 21th, 1985. In 2012, he obtained his master degree in Industrial Engineering and Management, with specialization Health Care Technology Management, at the University of Twente. He performed his graduation assignment at Ziekenhuisgroep Twente in Almelo. He studied the process design of an integrated emergency post.

After finishing his master studies, Nardo joined the department Industrial Engineering and Business Information Systems of the University of Twente for a $\mathrm{PhD}$ program. Additionally, he conducted research at HagaZiekenhuis in The Hague. The results of his studies are presented in this thesis. 



\section{List of publications}

N.J. Borgman, I.M.H. Vliegen, and E.W. Hans. Urgent care planning and scheduling in hospitals: A literature review. Working paper.

N.J. Borgman, M.R.K. Mes, I.M.H. Vliegen, and E.W. Hans. Improving the design and operation of an integrated emergency post via simulation. Journal of Simulation, 9(2):99-110, 2015.

N.J. Borgman, I.M.H. Vliegen, R.J. Boucherie, and E.W. Hans. Appointment scheduling with unscheduled arrivals and reprioritization. Flexible Services and Manufacturing Journal, 2017.

N.J. Borgman, I.M.H. Vliegen, and E.W. Hans. Emergency OR or not: A simulation study of emergency surgery scheduling policies Working paper.

N.J. Borgman, I.M.H. Vliegen, and E.W. Hans. Surgical procedure type scheduling incorporating semi-urgent patients Working paper. 